\title{
Pre-competition and recovery strategies in elite sport: the use of vascular occlusion and ischemic preconditioning
}

Natalie Williams
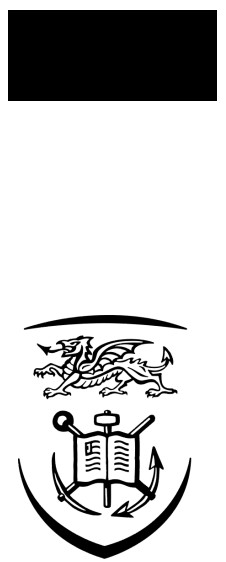

Swansea University

Prifysgol Abertawe

Submitted to Swansea University in fulfilment of the requirements for the Degree of Doctor of Philosophy

Swansea University 


\section{Summary}

Pre-competition and recovery strategies in elite sport: the use of vascular occlusion and ischemia

Understanding competition demands and training are only two components that impact overall performance which have been previously established in the literature; pre-competition and recovery can also improve athletic performance. However, considerably less research has been conducted in elite populations, investigating the effect of pre-competition and recovery strategies. This thesis set out to investigate a pre-competition and recovery strategy for application in elite sport, via a series of studies specifically focussed on vascular occlusion and limb ischemic preconditioning (IPC). Common to all studies was the importance of individualising cuff pressure and ensuring all studies were completed with elite athletes. Study one investigated the use of vascular occlusion combined with resistance exercise, performed in the morning prior to afternoon exercise performance. Despite exercise with occlusion attenuating the decline in $\mathrm{T}$ from morning to afternoon, this did not translate into improved afternoon performance in strength-power athletes. Study two examined the use of limb IPC applied $2 \mathrm{~h}$ and $24 \mathrm{~h}$ prior to swimming time trial performance. There were no differences in physiological (blood gases and lactate) or performance markers measured between conditions, when compared to a control. Study three investigated vascular occlusion applied as a recovery strategy following repeated sprint exercise, in rugby union players. Recovery rate, assessed through biochemical, hormonal, neuromuscular and subjective perception of muscle soreness were not significantly different following application of vascular occlusion. This thesis successfully examined the use of vascular occlusion/limb IPC as a precompetition and recovery strategy within elite athletes. Despite the absence of performance improvements, this thesis addressed some of the practical limitations of applying pre-determined protocols in sport and offered alternative protocols to be applied across a range of sports (strengthpower, anaerobic, team sports) in areas which have not previously been investigated, providing clear practical implications and future research to inform coaches and performance scientists.

\section{KEYWORDS:}

Pre-competition, recovery, ischemia, occlusion, athletic performance 


\section{Declarations and statement}

\section{Declaration}

This work has not previously been accepted in substance for any degree and is not being concurrently submitted in candidature for any degree.

Signed (candidate)

Date

\section{Statement 1}

This thesis is the result of my own investigations, except where otherwise stated. Where correction services have been used, the extent and nature of the correction is clearly marked in a footnote(s).

Other sources are acknowledged by footnotes giving explicit references. A bibliography is appended.

Signed (candidate)

Date

\section{Statement 2}

I hereby give consent for my thesis, if accepted, to be available for photocopying and for inter-library loan, and for the title and summary to be made available to outside organisations.

Signed (candidate)

Date 


\section{Table of Contents}

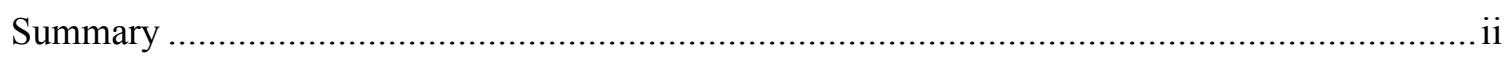

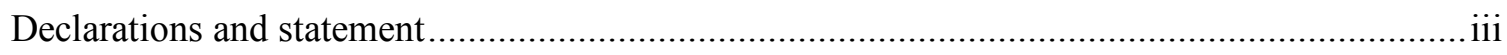

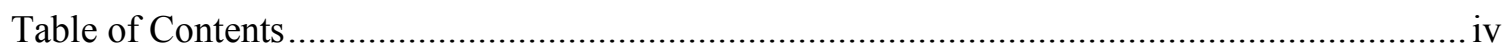

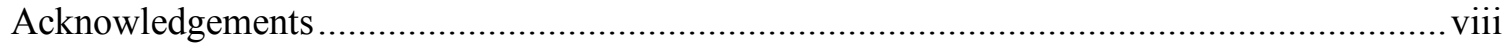

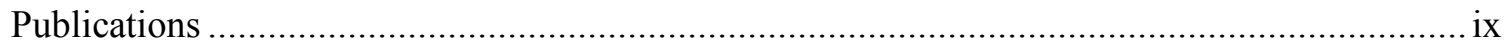

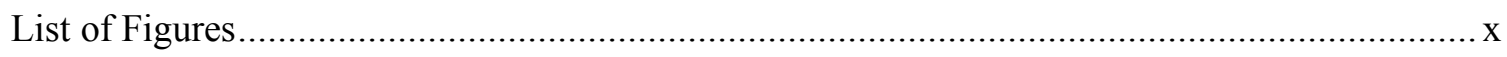

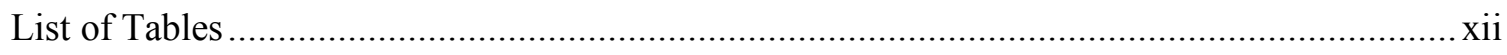

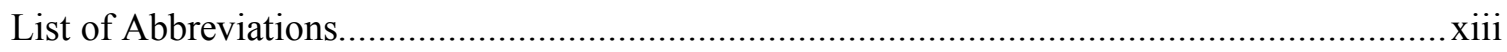

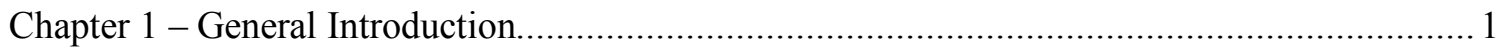

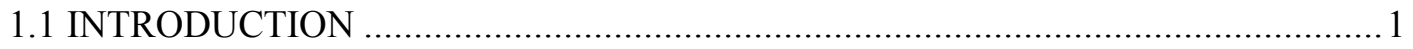

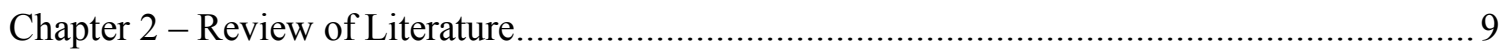

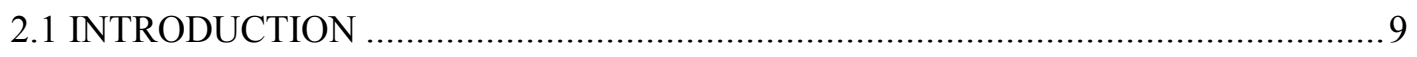

2.2 PRE-COMPETITION AND RECOVERY STRATEGIES IN ELITE SPORT ............ 10

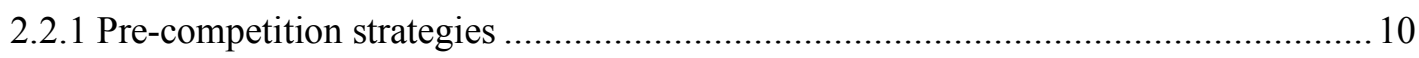

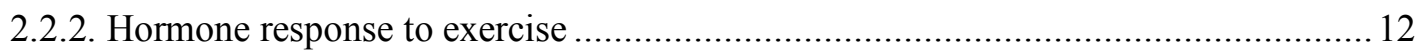

2.2.3 Circadian rhythmicity and morning exercise ....................................................... 18

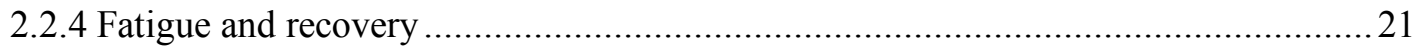

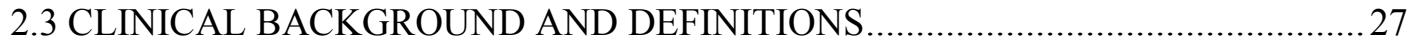

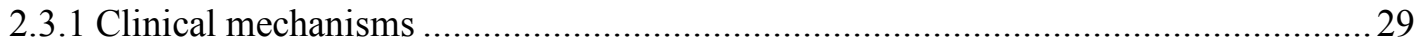

2.3.2 Introduction to IPC and vascular occlusion in sport performance .............................35

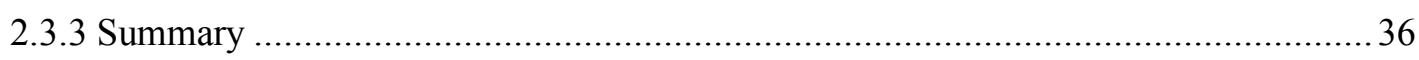

2.4 PRE-COMPETITON: THE USE OF VASCULAR OCCLUSION AND LIMB IPC.. 38

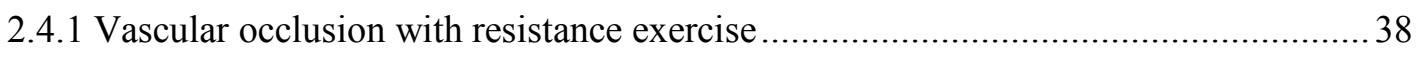

2.4.2 Identified mechanisms for the use of vascular occlusion and resistance exercise ...... 44

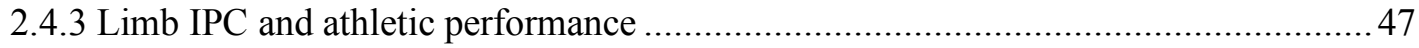

2.4.4 Identified mechanisms for the use of IPC as a pre-competition strategy ...................50

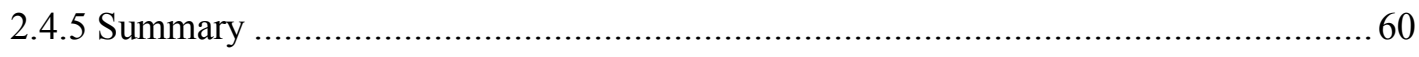

2.5 USE OF VASCULAR OCCLUSION FOR RECOVERY …......................................61

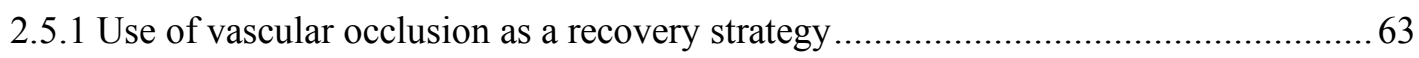

2.5.2 Proposed mechanisms for the use of vascular occlusion as a recovery modality ....... 63

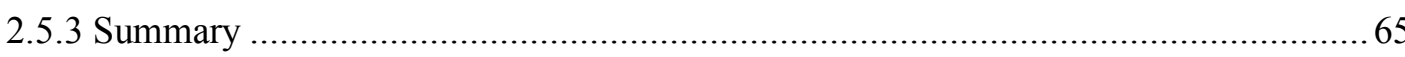

2.6 VARIABLES OF LIMB IPC AND VASCULAR OCCLUSION ….............................66 
2.6.1 Cuff Pressure 66

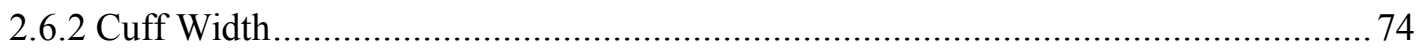

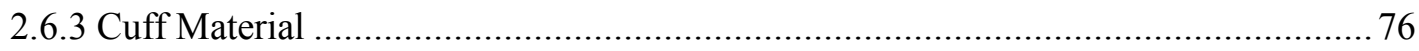

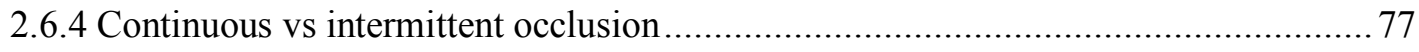

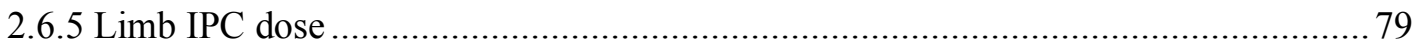

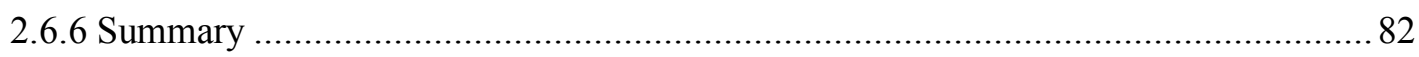

2.7 METHODLOGICAL CONSIDERATIONS .................................................. 82

2.7.1 Saliva as a diagnostic tool in sport and exercise ............................................. 82

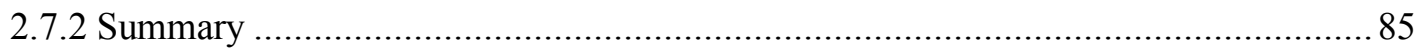

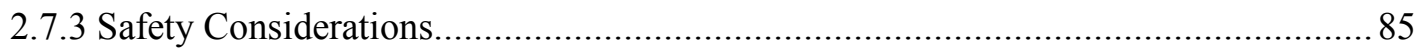

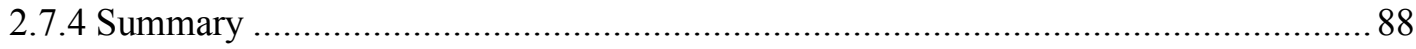

2.8 CONCLUSIONS AND RECOMMENDATIONS ......................................... 88

Chapter 3 - General methods ..................................................................... 91

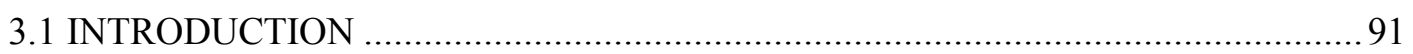

3.2 PARTICIPANTS ................................................................................. 91

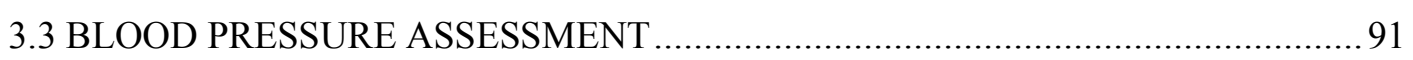

3.4 OCCLUSION CUFFS AND PRESSURE.................................................... 92

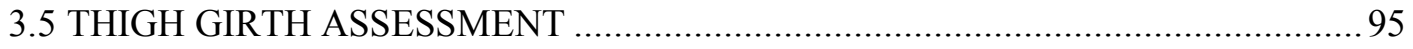

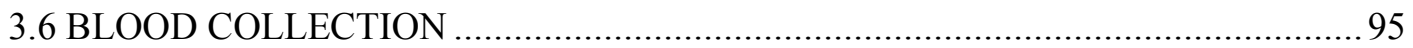

3.6.1 Blood Lactate.................................................................................... 95

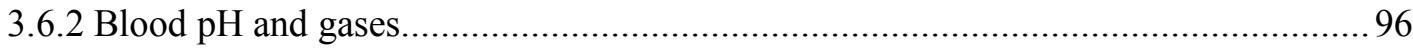

3.6.3 Creatine Kinase................................................................................ 97

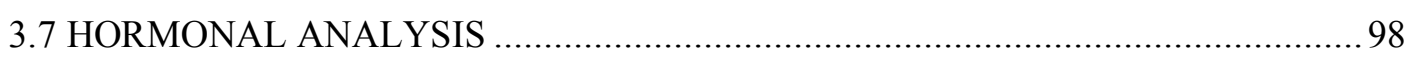

3.8 NEUROMUSCULAR PERFORMANCE ....................................................... 99

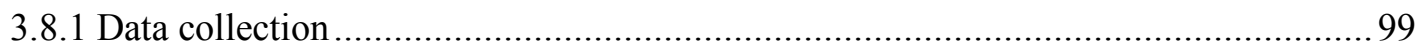

3.8.2 Determination of body weight ........................................................... 100

3.8.3 Countermovement jump ................................................................ 100

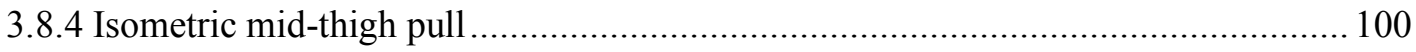

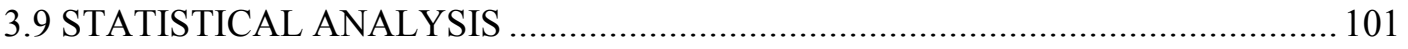

Chapter 4 - The effects of morning exercise with vascular occlusion on afternoon performance

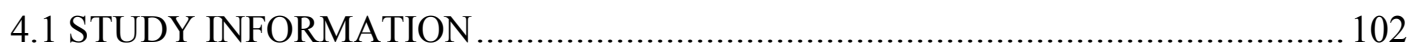

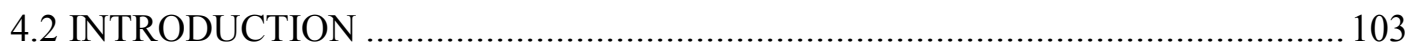

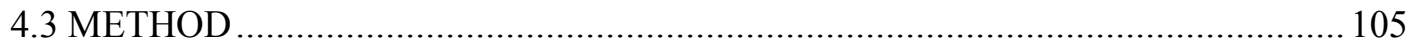

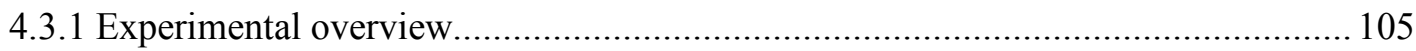

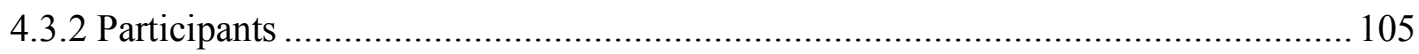


4.3.3 Main trial procedures 106

4.3.4 Statistical analysis.

4.4 RESULTS

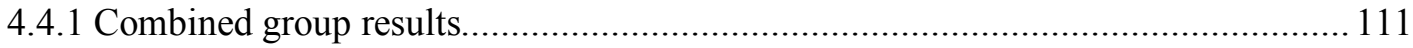

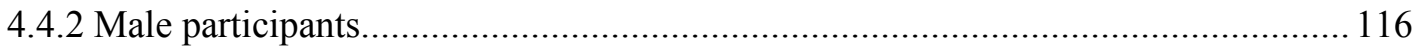

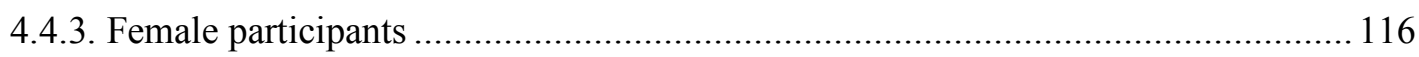

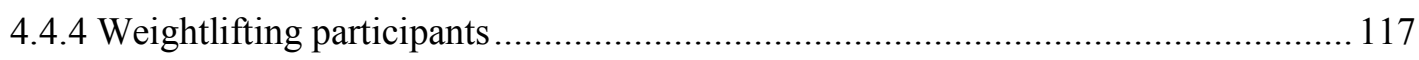

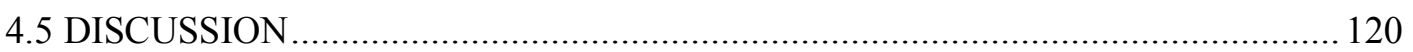

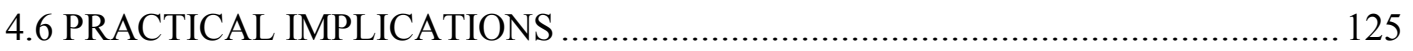

4.7 CONCLUSION

Chapter 5 - The effect of ischemic preconditioning on maximal swimming performance...

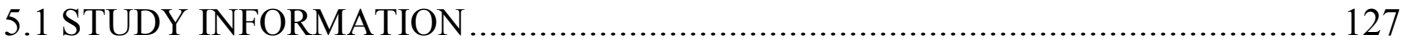

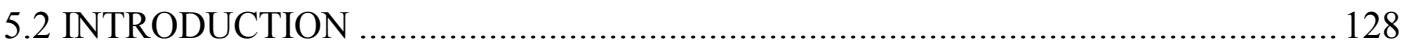

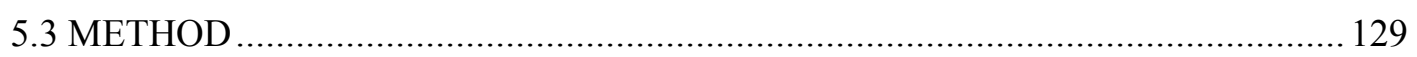

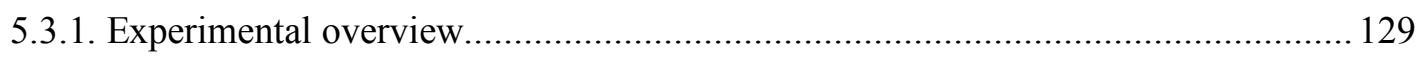

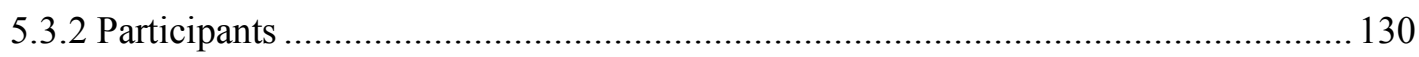

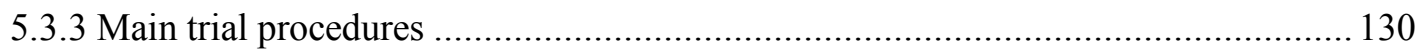

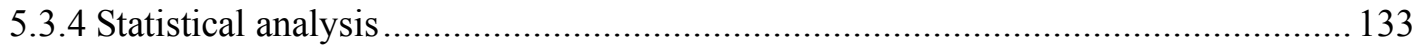

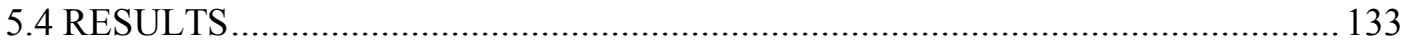

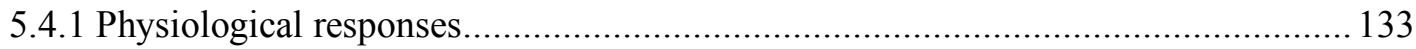

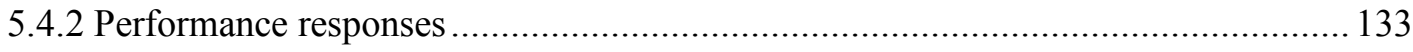

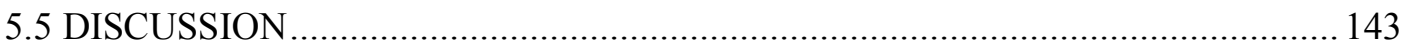

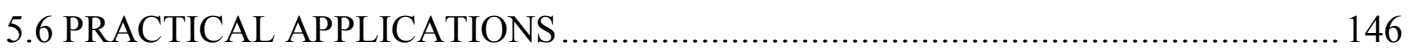

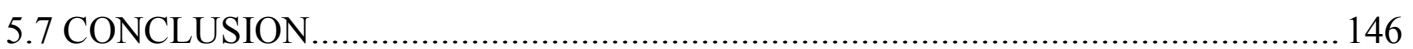

Chapter 6 - The effect of lower limb occlusion on recovery following sprint exercise in academy rugby union players

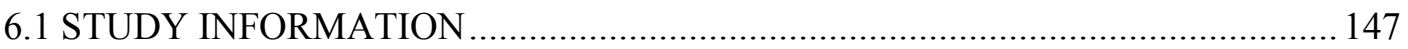

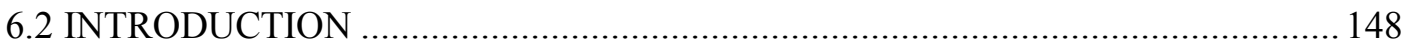

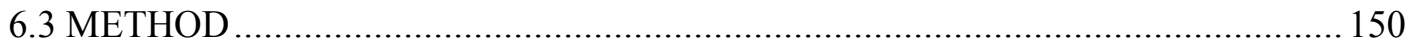

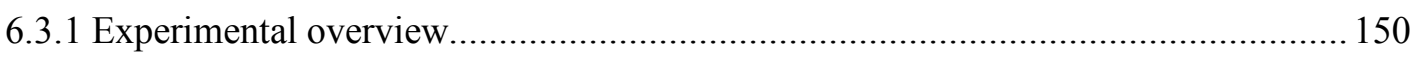

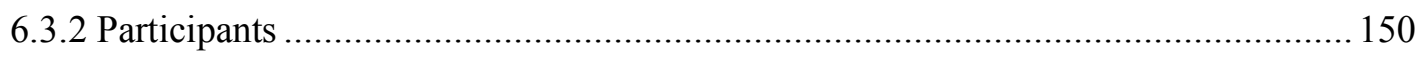

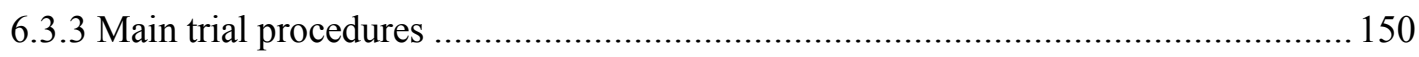

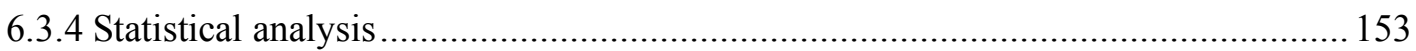

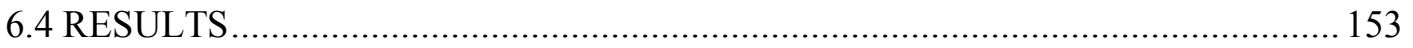

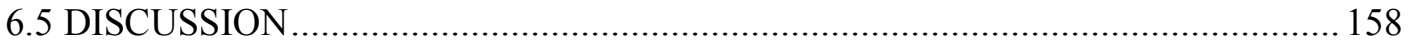

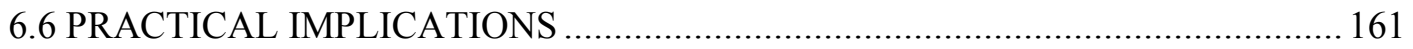




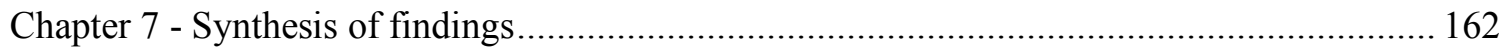

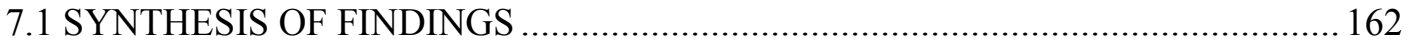

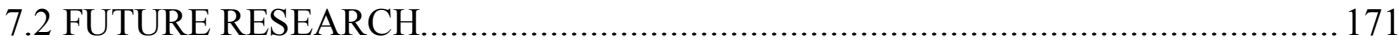

7.3 SUMMARY OF PRACTICAL IMPLICATIONS............................................... 172

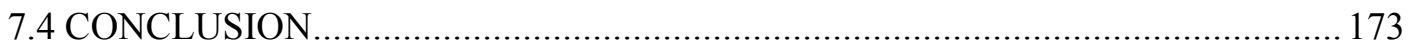

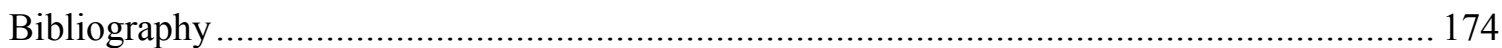

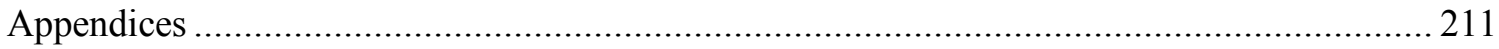

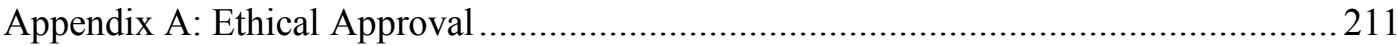

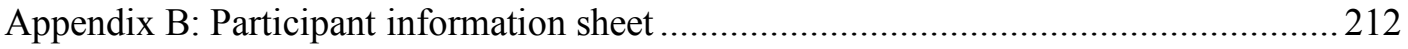

Appendix C: Consent form and health screening questionnaire .................................... 220

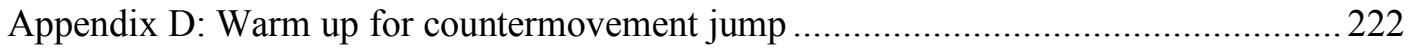

Appendix E: Warm up prior to swimming time trial performance ................................223

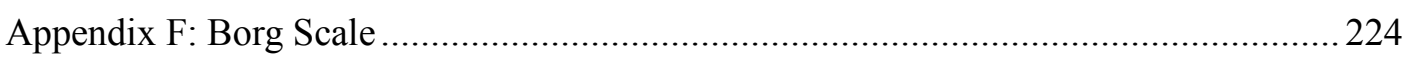

Appendix G: Perception of muscle soreness likert scale..................................................22 225

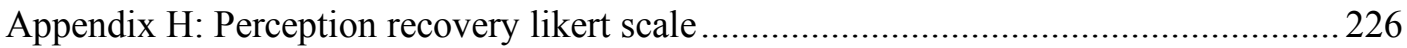




\section{Acknowledgements}

First, I would like to thank my supervisor, Prof. Liam Kilduff for his guidance and support throughout this $\mathrm{PhD}$, your knowledge and commitment has motivated and pushed me to develop beyond the fundamentals of completing this thesis. Dr Mark Russell, thank you for critically reviewing my work and enabling me to not only achieve writing my thesis but successfully submitting for publication, and Prof Christian Cook for knowledge, time and expertise. I would also like to thank Prof. David Shearer for his time and assistance with data analysis, alongside providing guidance and general support.

Sport Wales, thank you for the financial support provided throughout my $\mathrm{PhD}$, without this support it would not have been possible to achieve this within 3 years and hopefully I have imparted knowledge from my thesis to enable this to be applied in practice within Welsh Sport. Special thanks to the strength and conditioning team for helping with pilot studies, providing both challenge and support of my initial ideas and the lab techs for your help especially the last-minute equipment requests!

Thank you to the coaches and athletes of Welsh weightlifting and athletics, Bath and Manchester swimming and Cardiff Blues and Scarlets for your support and cooperation during organisation and completion of data collection.

My friends and family, thank you for all of your help and support. My mum, dad and sister Beky, who have continually supported me, helped and provided motivation through times of wanting to give up, but also throughout all of my studies to enable me to even consider completing a $\mathrm{PhD}$, I will always be grateful for your support and endless hugs, it really did make all the difference.

Finally, and, importantly, a massive thank you to my fiancé Sam, for providing endless support and encouragement, for putting up with me during the data collection and writing, I know how unbearable my company must have been during this time, but you were always there and provided me with adventures and a balance to enable me to continue enjoying and succeeding in this process, without your love and support I would not have achieved this without you. 


\section{Publications}

Williams, N., Russell, M., Cook, C.J. and Kilduff, L.P. (2018). The effect of ischemic preconditioning on maximal swimming performance. Journal of Strength and Conditioning Research, [Epub ahead of print].

Williams, N., Russell, M., Cook, C.J. and Kilduff, L.P. (2018). The effect of lower limb occlusion on recovery following sprint exercise in academy rugby players. Journal of Science and Medicine in Sport, [Epub ahead of print]. 


\section{List of Figures}

Figure 2.1 Dynamic changes of salivary estradiol, progesterone and testosterone during one menstrual cycle in young healthy women (Adapted from Celec et al. 2009).

Figure 2.2 Mechanisms of the early and delayed (late) phase of ischemic preconditioning (adapted from Koch et al. 2014)

Figure 2.3 Potential mechanistic pathways underlying remote ischemic preconditioning (extracted from Hausenloy \& Yellon 2008)

Figure 2.4 The mechanisms associated with each experimental chapter and application of vascular occlusion or limb IPC as pre-competition and recovery strategies

Figure 2.5 The percentage of arterial occlusion applied when using vascular occlusion with absolute and relative pressures (bSBP - brachial systolic blood pressure). Data from Loenneke et al. (2015), extracted from Jessee et al. (2016).

Figure 2.6 Prescription variables used in practice by performance scientists to implement limb ischemic preconditioning, identifying cuff width, restriction pressure and duration of applied pressure. (RES - resistance exercise; AER - aerobic exercise; PAS - passive) (From Patterson \& Brandner 2017) 81

Figure 3.1 Application of occlusion cuffs whilst in a supine position 94

Figure 4.1: Timeline of data collection. Con; control: RE+VO; resistance exercise with vascular occlusion: RM; repetition maximum. Measurements: blood pressure $(\bullet)$, testosterone and cortisol $(\times)$, blood lactate $(\diamond)$, Countermovement jump \& isometric mid-thigh pull ( ) .

Figure 4.2: $\Delta$ Testosterone concentrations (mean $\pm \mathrm{SD}$ ) throughout the control (Con) and resistance exercise with vascular occlusion (RE+VO) condition. (A) All participants, (B) male participants only, (C) female participants only, (D) weightlifting only participants * indicates significant $(\mathrm{p}<0.05)$ delta change measured AM to PM.

Figure 4.3: Correlation between the change in blood lactate and Testosterone pre-to post-exercise

Figure 4.4: Individual response in performance of peak power output determined from countermovement jump for the control and occlusion conditions. (A) All participants, (B) male participants, (C) female participants

Figure 4.5: Individual response in performance of peak force determined from isometric midthigh pull for the control and occlusion conditions. (A) All participants, (B) male participants, (C) female participants

Figure 5.1: Overview of timeline of data collection. IPC - ischemic preconditioning, WU - warm up, TT - time trial, $(\diamond)$ blood sample, $(\square)$ ischemic preconditioning, $(\bullet)$ RPE 
Figure 5.2: Change in blood markers from pre-ischemic preconditioning (IPC) to post-IPC and Pre-time trial (TT) to post-TT. (A) Bicarbonate, (B) Base excess, (C) Blood lactate, (D) pH. * indicates significance difference at sampling time point $(\mathrm{p}<0.05)$

Figure 5.3: Change in blood markers from pre-ischemic preconditioning (IPC) to post-IPC and Pre-time trial (TT) to post-TT for $100 \mathrm{~m}$ (right) and 200m (left). (A) Bicarbonate, (B) Base excess, (C) Blood lactate, (D) $\mathrm{pH}$ * * indicates significance difference at sampling time point $(\mathrm{p}<0.05) 138$ Figure 5.4: Individual responses of swimming time trial for the combined group (A), $100 \mathrm{~m}$ (B) and $200 \mathrm{~m}(\mathrm{C})$. IPC - ischemic preconditioning....

Figure 6.1: Timeline of data collection. Con; control trial, Recovery; occlusion trial, RE; resistance exercise. Measurements: salivary testosterone, salivary cortisol $(\bullet)$, blood sampling for blood lactate and Creatine Kinase ( ), perception muscle soreness questionnaires ( $\bullet$ ), countermovement jump $(\diamond)$

Figure 6.2: Blood markers (A) Creatine Kinase (B) Blood lactate collected pre, post, $2 \mathrm{~h}$ and $24 \mathrm{~h}$ post sprint protocol $*(\mathrm{p}<0.001)$

Figure 6.3: Saliva markers (A) Testosterone, (B) Cortisol (C) T/C ratio, collected pre, post, $2 \mathrm{~h}$ and 24 h post sprint $*(\mathrm{p}<0.001)$ 156

Figure 6.4: Neuromuscular function variables determined from countermovement jump (A) peak power output, (B) jump height, collected pre, post, $2 \mathrm{~h}$ and $24 \mathrm{~h}$ post sprint $*(\mathrm{p}<0.001)$. 157 


\section{List of Tables}

Table 2.1 The influence of early morning physical activity on afternoon performance

Table 2.2: Characteristics of the studies examining the acute response of resistance exercise combined with vascular occlusion 2010-2017

Table 2.3 Characteristics of the studies identifying the effect of limb ischemic preconditioning on exercise performance

Table 2.4 The use of vascular occlusion as a recovery modality

Table 2.5: Summary of restrictive cuff pressures, cuff widths and times between vascular occlusion/limb ischemic preconditioning and performance (2015-2017 \& excluding reviews)-- 78

Table 3.1: Summary of participant characteristics for each study ------------------------ 93

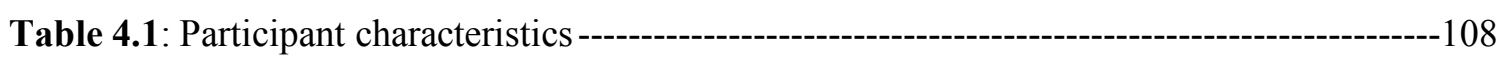

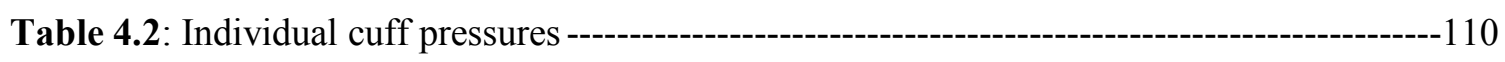

Table 4.3: Performance outcomes from countermovement jumps for the control and vascular occlusion conditions

Table 4.4: Performance outcomes from isometric mid-thigh pull for the control and vascular occlusion conditions $-119$

Table 5.1: Physiological responses across data collection time points, displayed for three conditions tested $-135$

Table 5.2: Performance variables from the swimming time trial (100 m, $200 \mathrm{~m}$ and combined) for the three conditions

Table 5.3: Change in results between control and ischemic preconditioning applied $2 \mathrm{~h}$ prior to performance when compared to 0.3 of the coefficient of variation to determine smallest worthwhile change $-141$

Table 5.4: Change in results between control and ischemic preconditioning applied $24 \mathrm{~h}$ prior to performance when compared to 0.3 of the coefficient of variation to determine smallest worthwhile change $-142$ 


\section{List of Abbreviations}

Acetyl-CoA - acetyl Coenzyme A

ACTH - adrenocorticotrophic hormone

ADP - adenosine diphosphate

AM - morning

AMP - adenosine monophosphate

ANOVA - analysis of variance

AOP - arterial occlusion pressure

ATP - adenosine triphosphate

BEecf - base excess

C - cortisol

$\mathrm{Ca}^{2+}$ - calcium

cAMP - cyclic adenosine monophosphate

CK - creatine kinase

CMJ - countermovement jump

CNS - central nervous system

Con - control

CV - coefficient variation

EIMD - exercise induced muscle damage

ELISA - enzyme-linked immunosorbent assay

EMG - electromyography

ES - effect size

F100ms - force at 100 milliseconds

FMD - flow mediated dilation

FSH - follicle-stimulating hormone

GnRH - gonadotrophin releasing hormone

GRF - ground reaction force

$\mathbf{H}^{+}$- hydrogen

$\mathrm{HCO}_{3}$ - bicarbonate

HPG - hypothalamic pituitary gonadal

ICC - intra-class correlation coefficients

IMTP - isometric mid-thigh pull

IPC - ischemic preconditioning

IRI - ischemic-reperfusion injury

JH - jump height

$\mathbf{K}^{+}$- potassium 
$\mathbf{K}_{\mathbf{A T P}}$ - ATP sensitive potassium

LH - lutenisng hormone

MANOVA - multivariate analysis of variance

NIRS - near infrared spectrometry

NMF - neuromuscular function

NO - nitric oxide

$\mathbf{O}_{2}$ - oxygen

$\mathbf{P i}$ - phosphate

$\mathbf{P C r}$ - phosphocreatine

PKC - protein kinase C

PM - afternoon

$\mathbf{P O}_{2}$ - partial pressure oxygen

$\mathbf{P C O}_{2}$ - partial pressure carbon dioxide

PPO - peak power output

PVN - paraventricular nucleus

$\mathbf{R E}+\mathbf{V O}$ - resistance exercise with vascular occlusion

RFD - rate of force development

RIPC - remote ischemic preconditioning

ROS - reactive oxygen species

RM - repetition maximum

SC - stroke count

SD - standard deviation

SCN - suprachiasmatic nucleus

$\mathrm{SO}_{\mathbf{2}} \%$ - percentage oxygen saturation

SR - stroke rate

$\mathbf{T}$ - testosterone

$\mathbf{T C O}_{2}$ - total carbon dioxide

TT - time trial

$\mathbf{V O}_{2}$ - volume of oxygen

$\mathbf{V}_{\max }$ - peak velocity 


\section{Chapter 1 - General Introduction}

\subsection{INTRODUCTION}

Coaches, performance scientists and athletes spend a significant amount of time trying to understand the demands of competition to ensure the training stimulus is appropriate and well planned to optimise competition performance. However, understanding competition demands and training are only two components that impact overall performance; the pre-competition environment, a window on the day of competition in which performance can be acutely enhanced, and recovery strategies, are two equally important areas that can improve athletic performance. To date, literature has established the demands of competition along with the impact and use of training to improve competition performance, however considerably less research has been conducted in elite populations in the areas of pre-competition and recovery, specifically that can be applied in practice; thus this thesis aims to add to the current body of literature to inform coaches and performance scientists of effective pre-competition and recovery strategies available which will consider the constraints on the day of competition and provide practical methods to implement with athletes.

Several pre-competition strategies have been identified that may positively impact performance, for instance a meta-analysis investigating the effects of warm up on physical performance highlighted improved performance in $79 \%$ of the criterions examined, with improvements ranging from 1-20\% (Fradkin et al. 2010). Despite this, 20\% of studies showed either no improvement or a detrimental effect, however results suggest this is due to an inappropriate warm up that was either not suited to the performance test to follow or the intensity, duration and recovery following warm up was insufficient to improve performance. This highlights the importance of optimising strategies that are available to be used prior to competition. More recently, Kilduff et al. (2013) have identified additional pre-competition strategies to optimise performance across different sports. These have included passive heat maintenance, post activation potentiation, prior morning exercise, hormonal priming and limb ischemic preconditioning (IPC).

Athletes can be susceptible to changes in their physical performance as a function of time (Cook et al. 2014); they need to ensure they can optimally perform at competition regardless of its timing. Opportunities exists on the day of competition to influence subsequent performance, $\mathrm{T}$, for instance, is important for sports performance as it can promote increases in voluntary exercise (Beaven et al. 2008; Crewther et al. 2014); athlete T levels have been positively correlated to selfselected workloads (Goto et al. 2007) whilst greater $\mathrm{T}$ responses to competition have been 
associated with higher motivation to win (Hellhammer et al. 1985), thus fluctuations in T could be a major factor determining competition performance. However, $\mathrm{T}$ exhibits circadian rhythms in basal levels, which are elevated in the morning with a nadir in the late afternoon (Kanaley et al. 2001; Kraemer et al. 2001). Previous literature has therefore investigated the use of prior morning exercise, aiming to reduce the circadian decline in $\mathrm{T}$ and optimise afternoon performance. For illustration, Cook et al. (2014) have reported morning strength training was related to improvements in countermovement jump (CMJ) peak power output (PPO) and sprint times $6 \mathrm{~h}$ later, associated with an offset in circadian decline in testosterone (T).

Limb IPC, or vascular occlusion, is another emerging strategy that has received attention for its use in training, however, less research has established its application as a pre-competition strategy. Some positive effects on performance have been identified, improving $5 \mathrm{~km}$ running time trial (TT) (Bailey et al. 2012), $50 \mathrm{~m}$ (Lisbôa et al. 2017) and $100 \mathrm{~m}$ swim time (Jean-St-Michel et al. 2011) when used as a pre-competition strategy. However, the majority of studies have investigated the use of limb IPC in un-trained participants (Marocolo et al. 2016). Ischemia refers to the restriction in blood supply to a tissue, causing a shortage of oxygen $\left(\mathrm{O}_{2}\right)$ required for metabolism (Kalogeris et al. 2012). Considering the potential application across different sports, in combination with the relative ease of use and minimal required equipment, limb IPC or occlusion provides a good option to implement as a warm up in elite sport. The original concept of IPC aimed to reduce myocardial infarct size by applying brief ischemia and reperfusion to an organ or tissue and provide protection against subsequent lethal ischemia-reperfusion injury (IRI). This was extended and is a process used to protect cardiac and skeletal muscle against IRI (Zhao \& Vinten-Johansen 2006) (further detail see section 2.3.1). Within sport, this strategy has involved applying a tourniquet, pressurised cuff or elastic band to a specific limb to restrict blood flow. The aim of external pressures is to maintain partial arterial inflow to the muscle while occluding venous return (Pope et al. 2013) resulting in a pooling of venous blood around the exercising muscle when occlusion is combined with exercise (Fahs et al. 2012) or create cycles of ischemia and reperfusion when applied during passive rest. Typically, limb IPC involves 3-4 cycles of five min occlusion and five min reperfusion whilst the athlete lies in a supine position at rest (Thijssen et al. 2016). Whereas when vascular occlusion is combined with exercise, occlusion of the limbs occurs for the duration of the exercise being performed either continuous or intermittent (see section 2.5.1). Manipulation of the protocol differentiates the use of blood flow restriction and associated physiological responses, altering mechanisms for application as a pre-competition strategy with varying application for strength and power, anaerobic specific events or team sports.

Despite previously identified benefits of completing morning exercise to improve performance through attenuations in the decline in $\mathrm{T}$, previous resistance exercise protocols may not be 
favourable to implement on the day of competition or for specific sports, for example, weightlifting would not complete $5 \times 10$ bench press in training or competition and therefore lacks practical application. However, as research has proposed the importance of an intensity and volume threshold to cause a metabolic response to initiate an acute T response (Cumming et al. 1987) an alternative mechanism may be required to allow a reduced volume of repetitions but still produce a metabolic and hormonal response. These sought-after strategies require investigation for implementation in elite sport, in which vascular occlusion provides an alternative option. Schoenfeld (2013) reported a greater accumulation of metabolites due to increased production in a more hypoxic state and limited removal due to occlusion acting as a primary moderator of metabolic response. When resistance exercise is combined with vascular occlusion it has shown to increase strength and hypertrophy; this is achieved with lower volumes/intensity of exercise, but has still provided a sufficient training stimulus when compared to traditional strength training (Slysz et al. 2016; Scott et al. 2015). One study of particular interest performed five sets of five repetitions of three resistance exercises at $70 \%$ one repetition maximum (1RM) for three weeks (Cook et al. 2014). Consistent with previous research, an increase in strength was identified, but also an occlusion-dependent elevation in salivary T (ES 1.50 to 2.19 ) immediately after exposure was highlighted (Cook et al. 2014), potentially resulting from increased metabolic stress (Suga et al. 2012) of vascular occlusion combined with resistance exercise.

Considering the potential role of $\mathrm{T}$ in mediating athletic performance, vascular occlusion has demonstrated use for stimulating hormone ( $T$; Cook et al. 2014) and also muscle fibre recruitment (Takarada et al. 2000; Yasuda et al. 2009) with much lower volumes (25 repetitions at 70\% 1RM) than in previous morning priming studies (i.e. 50 repetitions at 75\% 1RM) (Further details see section 2.2.3, Table 2.1). It has been suggested vascular occlusion with exercise causes an increased metabolic response due to restricted venous outflow; greater duration of metabolic acidosis via the trapping of and accumulation of intramuscular protocols $\left(\mathrm{H}^{+}\right)$and stimulation of metabaroceptors, possibly eliciting an exaggerated acute systemic hormonal response (Pope et al. 2013). This has not been previously investigated in males or females but as it offers application as a pre-competition strategy to offset the circadian decline in $\mathrm{T}$ and improve afternoon performance of strength and power sports such as weightlifting, it warrants further research.

To note, the majority of research relating hormone response and resistance exercise exists within males, however there is evidence of elevated $\mathrm{T}$ and self-selected workloads in females (Cook \& Beaven 2013); back squat performance correlated to individual $\mathrm{T}$ levels $(\mathrm{r}=0.7062 ; \mathrm{p}=0.0103)$, especially when considering the difference in elite versus non-elite females (Cook et al. 2012). Cardinale \& Stone (2006) observed a significant correlation between T and CMJ JH in elite women. With evidence supporting the potential benefit of $\mathrm{T}$ on performance in females, research 
has not previously investigated the effect of morning exercise on afternoon performance in female athletes and therefore warrants further examination.

Alternatively, occlusion applied whilst at passive rest before competition (i.e. limb IPC) is a strategy receiving more attention for its use in anaerobic and endurance competition performances. Within athletic performance, the benefits of limb IPC have been observed in time to exhaustion (De Groot et al. 2010), anaerobic specific performance tests (Jean-St-Michel et al. 2011) and repeated sprint ability (Patterson et al. 2015). Specifically in swimming, limb IPC has shown to be beneficial to performance, potentially owing to greater contribution of adenosine triphosphate (ATP) generated from the aerobic system following limb IPC which is important for 100 to $400 \mathrm{~m}$ swimming performance as reports have suggested 54.1-61.4\% aerobic contribution for $100 \mathrm{~m}$ and 83.2-85.5\% for 400m (Rodriguez \& Mader 2003). Jean-St-Michel et al. (2011) reported five min ischemia followed by five min reperfusion, repeated for four cycles, resulted in $1.1 \%$ improvement in $100 \mathrm{~m}$ performance relative to personal best times. Similarly, Marocolo et al. (2015) completed the same limb IPC protocol prior to performance, resulting in 1.46\% improvement in $100 \mathrm{~m}$ TT (further supporting research see section 2.5.3). It has been reported limb IPC induces acute vascular adaptations, due to the effect of adenosine, resulting in local vasodilation and enhanced blood flow (Tapuria et al. 2008). Consequently, enhanced functional sympatholysis may speed and increase $\mathrm{O}_{2}$ extraction by means of matching demand with supply (Horiuchi et al. 2015) facilitating aerobic contribution. Limb IPC can also cause a faster uptake of acetyl Coenzyme A (acetyl-CoA) by mitochondria thus maintaining lactate accumulation at a metabolically acceptable level due to greater contribution of aerobically generated ATP for exercise (Jean-St-Michel et al. 2011); this may reduce metabolic fatigue impacting performance (for further mechanism see section 2.5.4). Given the nature of swimming and controlled breathing, which can result in exercise induced hypoxemia, decreased $\mathrm{pH}$ and metabolic accumulation, a systemic increase in blood flow and $\mathrm{O}_{2}$ delivery could be speculated to be advantageous to performance.

These mechanisms are associated with the early phase of limb IPC and have been implemented within the 3-4 h window previously reported (Kuzuya et al. 1993). However, race day is frequently congested and restricted by designated warm up timings, strict marshalling regulations and additional factors such as race suits and psychological strategies; therefore, alternative options are investigated to impact swimming performance due to the marginal time differences for medal positions. As reported within the clinical literature, the application of IPC to harness a second or late phase appearing 12-24 h after application (Kuzuya et al. 1993) may have increased practical application. This has only been investigated in one previous study in sport, highlighting a protocol of four cycles of five min occlusion and five min reperfusion applied $1 \mathrm{~h}$ or $24 \mathrm{~h}$ prior to a $5 \mathrm{~km}$ TT did not improve performance. However, previous research in swimming has identified a 
consistent improvement in performance unlike that of $5 \mathrm{~km}$ running TT (e.g. Bailey et al. 2012; Tocco et al. 2015). The mechanisms associated may also have a greater impact for swimming due to the associated nature of the sport. Clinical reports suggest that even after the removal of adenosine it still infers a protective effect lasting up to $72 \mathrm{~h}$ (Loukogeorgakis et al. 2005) and appears dependent on gene expression and de novo synthesis of proteins involving endothelial, immune response and cellular energy metabolism (Koch et al. 2014). Unique to the late phase is the role of reactive oxygen species (ROS) triggering delayed protection, nitric oxide (NO) being both a trigger and a mediator in the late IPC and prevention of endothelial dysfunction normally associated with exercise due to altered neutrophil function; FMD was preserved when IPC was applied $24 \mathrm{~h}$ prior to exercise $(8.7 \%$ before and $8.4 \%$ after) (Loukogeorgakis et al. 2005). Improved endothelial function and prevention of decline in FMD improve aerobic contribution to performance (Dawson et al. 2013; Bailey et al. 2012). The evidenced benefit of limb IPC for swimming performance makes it an attractive strategy to apply in elite sport, but the possibility of applying limb IPC $24 \mathrm{~h}$ would improve practicality and prevent additional requirements in an already busy schedule on the day of competition and therefore warrants further investigation in elite swimming performance.

As specified, the importance of recovery and application of recovery strategies is also a key component that can improve athletic performance during ever increasing congested competition schedules. Acute and chronic exercise induced physiological adaptations, mechanical and biochemical changes occur in response to exercise which cause fatigue, this includes both central and peripheral fatigue (Bishop et al. 2008). Short term fatigue resulting from athletic performance has been negatively linked to subsequent performance (Montgomery et al. 2008), injury (Bishop et al. 2008) and even illness (Cunniffe et al. 2011). Recovery has therefore become an integral part of training and competition to optimise performance. Accordingly, various methods are used in applied practice to indicate the presence of exercise induced muscle damage (EIMD) and fatigue to inform recovery required following competition before successive workouts or indicate readiness for the next training session (Ferguson et al. 2014; Bishop et al. 2008). Specifically within team sport, elevated Creatine Kinase (CK) (Cunniffe et al. 2010; Russell et al. 2016), disruption in $\mathrm{T}$ and cortisol (C) hormonal milieu (Shearer et al. 2015) and impairments in neuromuscular function (NMF) have been measured to identify responses which occur post-match (West et al. 2014) and have shown to be disrupted for at least $48 \mathrm{~h}$ (West et al. 2014; Russell et al. 2016). Since multiple games are played in the week ( $<72 \mathrm{~h}$ separating games) recovery strategies are integral components in the weekly training regimen to prevent any negative effects on performance. However, since the causes of fatigue are varied, depending on the nature of exercise, there are several approaches to restoring homeostasis of the muscle cell (Bishop et al. 2008). Numerous recovery strategies have been reported in the literature; results of a meta-analysis by 
Nédélec et al. (2013) highlighted cold water and contrast therapy ( $88 \%$ teams), active recovery (81\%), massage (78\%), stretching (50\%), compression garments $(22 \%)$ and electrical stimulation $(13 \%)$ were the recovery strategies used in team sport. However, many of these strategies have limitations for application due to required equipment, space, time and cost, with literature also reporting contrasts in the effectiveness of each of these strategies. Ice baths for instance decrease muscle temperature which may be negative for multiple efforts or games performed in one day of competition.

Acknowledging the importance of recovery as one component to improve performance, additional recovery strategies are sought after. Recently, vascular occlusion, the use of blood pressure cuffs applied to specific limbs, whilst in the supine position, to restrict blood flow, has become more prevalent as a recovery strategy (Beaven et al. 2012; Page et al. 2017). However, there are many unanswered questions and limited investigations to date, with inconsistencies in the current literature. Vascular occlusion follows a similar protocol for use as a recovery strategy as that discussed for limb IPC. Other than timing of application, the athlete is rested in the supine position when cuffs are applied, inflated and deflated for cycles of occlusion (ischemia) and reperfusion. Variances exist between continual and intermittent occlusion within the recovery literature (see section 2.6) with further differences in duration of occlusion; successful studies have implemented two cycles of three min occlusion and three min reperfusion and three cycles of five min occlusion and five min reperfusion (Beaven et al. 2012; Page et al. 2017). These two studies (out of five investigating occlusion for recovery; see section 2.6 for all studies) have found a benefit of vascular occlusion on recovery rate (Beaven et al. 2012; Page et al. 2017), however, the exact mechanisms of how this strategy improved performance are debated. Reports suggest vascular occlusion raises the level of adenosine, causing an increase in blood flow and thus $\mathrm{O}_{2}$ and nutrient delivery due to the associated dilation of vasculature, believed to be exaggerated in the reperfusion phase once the cuff pressure is released (see section 2.6.2 for further detail). Alternatively, attenuated inflammatory response (Konstantinov et al. 2004) and reduction of muscle oedema and intramuscular pressure decrease nocioreceptor stimulation, potentially reducing muscle soreness (Howatson \& van Someren 2008). Page et al. (2017) identified increased recovery of CK following vascular occlusion. However, only one indicator of recovery and research completed in un-trained participants reduces practical application, therefore, replication of current research protocols in elite athletes with physiological markers is required to identify the efficacy of occlusion as a recovery strategy.

While some research has been completed into the use of limb IPC and vascular occlusion for precompetition and recovery, there are still numerous unanswered questions in multiple areas of this research area, however specifically for the focus of this thesis, there are many unanswered 
questions on practicality and application for elite athletes spanning a range of sports. A broad question links to participants and methodological protocols; limited research has investigated the use of limb IPC or vascular occlusion in elite athletes, a large majority of literature is reported in healthy un-trained males, which has limited application in elite sport as it is difficult to transfer a possible positive effect to an elite population due to differences in physiological responses in comparison to un-trained participants (Marocolo et al. 2016). For example, statistically significant differences have been observed in systolic blood pressure and heart rate both before and after exercise $(p<0.001)$ between trained and un-trained males. Trained athletes are able to increase their systolic blood pressure with relatively lower heart rates as compared to untrained individuals (Vandavasi \& Sukumar 2016). Furthermore, research has suggested a faster recovery of muscle function when compared to an untrained group (Newton et al. 2008) and therefore efficacy of recovery strategies may vary between trained and untrained individuals.

Within elite sport it is essential all strategies are individualised, however previous research has implemented a standardised cuff pressure despite research acknowledging the same pressure may not restrict blood flow similarly in each individual, especially since the amount of tissue surrounding the blood vessels influences the pressure exerted on the vasculature and impacts the degree of blood flow restriction (Hargens et al. 1987). Thicker skin and subcutaneous fat in the thighs may act as a barrier between the occlusion cuffs and circulatory pathways resulting in low or no change in the diameter of the targeted blood vessels and minimising overall effect of external pressure (Karabulut \& Perez 2013). It is likely the degree of intramuscular pressure depends on the architecture features of the muscle (Hunt et al. 2016) with hypertrophied muscles having an increased volume of tissue than fat and therefore less pressure is required to restrict the vasculature (Wernbom et al. 2006). Initial investigations suggested pressures to restrict blood flow of the lower limbs should be determined by limb circumference for both sexes (Loenneke et al. 2012) with more recent research supporting this finding, however, research has identified brachial blood pressure may be an additional significant predictor (Loenneke et al. 2015). Therefore, to ensure practical application and individualisation for elite athletes to improve performance, this is an area requiring further investigation to determine application in elite sport. There are also three very specific areas where questions are unanswered relating to elite sport, potentially offering application on the day of competition due to protocols and associated mechanisms identified in Figure 2.4 Section 2.3. Firstly, the use of vascular occlusion combined with exercise performed the morning prior to afternoon performance has not received any attention despite hormonal response observed previously (Cook et al. 2014) when performing a lower volume of resistance exercise than adopted in morning priming strategies (Russell et al. 2015), with the lower volume of exercise favourable for athletes to complete prior to competition. Secondly, only one study has researched the effect of the delayed phase of IPC on performance, with potentially excellent 
practical application as the timings would not be restricted by competition day timelines of strict marshalling regulations and warm up timings for example, in addition to the consistent supportive research of the use of limb IPC to improve swimming performance, it may be an attractive strategy for elite athletes and coaches. Thirdly, vascular occlusion provides a practical strategy for recovery due to limited required equipment and space, suitable for use when travelling away, however, research is contrasting and minimal in elite athletes providing too many questions at present to implement within elite sport.

The research questions and practical application for elite sport were designed in accordance to the models proposed by Jones et al. (2017) and Coutts (2016) in which strategies have been designed to undertake and integrate research into practice, suggesting the researcher to develop not inform practice, as development is more synonymous with a successful integrated research practice model (Jones et al. 2017). The models are built upon Kahneman 2011 'Thinking, Fast and Slow' and Pasteur's Quadrant 2007, developing a continuum of research and practice, assessing 'on-field' and 'off-field' brains to develop practice (Jones et al. 2017).

Therefore, the overall aims of this thesis were to assess the use of vascular occlusion and limb IPC for use pre-competition and recovery across elite athletes, both individual and team sports, implementing individualised cuff pressures for all strategies. The specific objectives to be realised:

i. Investigate the use of vascular occlusion during resistance exercise in the morning $(5 \mathrm{~h}$ prior) as a priming strategy to improve afternoon strength/power performance including identifying hormone response and the difference in response between sexes (Chapter 4)

ii. Investigate the use of limb IPC applied in the early ( $2 \mathrm{~h}$ ) and late (24 h) phase on swimming performance (Chapter 5)

iii. Investigate the use of vascular occlusion as a recovery strategy following sprint exercise in team sport (Chapter 6) 


\section{Chapter 2 - Review of Literature}

\subsection{INTRODUCTION}

The purpose of this review was to evaluate and appraise current research that examined the use of vascular occlusion and limb IPC on exercise performance when used as pre-competition and recovery modalities; the following review is separated into sub-sections. Section 2.2 provides an overview of the theoretical background for the use of pre-competition in elite sport, providing discussion around potential pre-competition strategies and associated physiological mechanisms, inclusive of hormone response. This will be followed by recovery strategies used in elite sport and a rationale for how this can positively impact athletic performance.

Section 2.3 provides a review of vascular occlusion and limb IPC, a definition and the clinical background to establish the origin of this strategy, providing context to the following sections. This is followed by section 2.4 and 2.5 which will discuss the impact of vascular occlusion and limb IPC on athletic performance, split into two sections of pre-competition and recovery, respectively. Within each section, current literature available will be reviewed along with reported mechanisms impacting performance. These sections aim to demonstrate that research to date has numerous questions regarding the application and protocol of limb IPC and vascular occlusion and where limitations lie regarding implementation in elite sport. The following criteria was used to determine research reviewed and the following criteria excluded articles from this section of the review: (i) articles that were review papers, (ii) articles that did not report outcomes directly relating to exercise performance, (iii) articles that examined sedentary participants or included participants under 18 or over 40 years (iv) articles that examined multiple or repeated (daily) application of limb IPC and vascular occlusion.

Section 2.6 will discuss variables of blood flow restriction, including cuff pressure and thus will aim to provide rationale for the methodology for limb IPC and vascular occlusion in the experimental chapters. Section 2.7 will discuss methodological considerations of saliva as a medium to measure hormone response along with safety considerations of vascular occlusion, before providing conclusions in section 2.8. 


\subsection{PRE-COMPETITION AND RECOVERY STRATEGIES IN ELITE SPORT}

Coaches, performance scientists and athletes spend a significant amount of time understanding competition demands and ensuring training is appropriate and well planned to result in optimal performance at competition. However, training is only one component impacting performance, alongside competition itself, pre-competition and recovery are two keys areas that can improve athletic performance.

\subsubsection{Pre-competition strategies}

Pre-competition strategies can acutely enhance performance with appropriately designed warm up, passive heat maintenance, post activation potentiation, prior exercise and hormonal priming and limb IPC all reported as strategies implemented within elite sport (Kilduff et al. 2013).

The pre-event warm up is a well-established practice preceding athletic events (Pearce et al. 2012), acting as a means to prepare the body for impending training or competition (Sporer et al. 2012). Evidence to support the positive aspects of an active warm up on performance is extensive, with coaches and athletes supporting this view from a practical perspective; prior physical activity is considered a vital component of preparation for optimal performance (Houmard 1991). A metaanalysis investigating the effects of warm up on physical performance highlighted enhanced performance in $79 \%$ of the criterions examined, with the degree of improvement ranging from 1$20 \%$ (Fradkin et al. 2010), the importance of completing warm up is therefore evident within competitive sport. A recent report has also shown 16 of 21 outcome pairings highlighted a positive effect of a high-load dynamic warm up to enhance power and strength performance (McCrary et al. 2015). Despite this, Fradkin et al. (2010) highlighted 20\% of studies showed no improvement or a detrimental effect consequence of either an inappropriate warm up not suited to the performance to follow, intensity and duration were insufficient or the time delay between warm up and performance prevented improved performance (e.g. West et al. 2013) .

The theoretical intention behind the interest afforded to warm up is to reduce the potential risk of injury and enhance performance by increasing muscle temperature and/or core temperature (Bishop 2003). Previous literature has documented the beneficial effects of an active warm up are the consequence of temperature dependent physiological processes; this is outside the scope of this review, for further detail refer to Bishop (2003). Research has also observed that the physiological responses to warm up may not be solely related to temperature and in fact can be categorised into temperature and non-temperature related factors (Bishop 2003). For example, existing evidence suggests that temperature alone is not deterministic of the volume of oxygen $\left(\mathrm{VO}_{2}\right)$ response and subsequent performance (Burnley \& Jones 2007). Therefore, the concepts of 'priming' and 'prior 
exercise' are appropriate in describing strategies available to coaches and performance scientists to implement prior to competition to improve performance through physiological mechanisms other than those related to temperature (Bishop 2003). The proposed benefits of priming are to prepare the energy systems involved in $\mathrm{O}_{2}$ transport and utilisation, allowing the athlete to reach a higher level of aerobic metabolism more rapidly (Bishop et al. 2001; Bishop et al. 2003). Additional physiological (metabolic, biochemical, neuromuscular) benefits of priming exercise have been reported to improve performance, for example priming exercise seems to increase blood flow, oxygenation, oxidative enzyme activity and electromyography (EMG) activity and accelerate the overall $\mathrm{VO}_{2}$ response (Jones et al. 2003). Ingham et al. (2013) reported an increase of $1.84 \mathrm{mmol} \cdot \mathrm{L}^{-1}$ in blood lactate and $1.43 \mathrm{~s}$ faster $800 \mathrm{~m}$ running performance following prior exercise. It was reported the intervention increased the speed of $\mathrm{O}_{2}$ kinetics and spared the anaerobic metabolism (Intervention: 68\% aerobic and 32\% anaerobic contribution; Control: 62\% aerobic and 38\% anaerobic contribution). Higher initial rates of ATP hydrolysis have also been associated with prior exercise and possible influences on performance (Bailey et al. 2011).

An alternative priming strategy has been termed post activation potentiation, this is a method reported to improve muscle performance following a pre-loading stimulus due to an increase in muscle twitch force or H-reflex amplitude following prior contraction (Gullich \& Sehmidtbleicher 1996; Trimble \& Harp 1998). The mechanisms of muscle potentiation and fatigue co-exist and any resulting performance benefits are dependent on the balance between these two factors (Kilduff et al. 2013); the optimal recovery time to maximise the effect of post activation potentiation on PPO has been reported between 8 and 12 min for the lower body (Kilduff et al. 2007; Kilduff et al. 2008; Crewther et al. 2011; Seitz et al. 2015). Therefore, as with active warm up, relatively close proximity (10-20 $\mathrm{min})$ of completion prior to competition is required, which may be limited by logistics and strict marshalling regulations at competition.

Significant barriers and challenges are present within sport, preventing the completion of optimal pre-competition (priming) routines; this includes time delays, the environment and logistics of sporting competition (Sporer et al. 2012). However, there is opportunity for coaches and athletes to apply several pre-competition strategies and optimise alternative physiological aspects other than temperature-related mechanisms to improve performance, for instance limb IPC may be applied to increase blood flow prior to performance, with reports suggesting application 15 min to $8 \mathrm{~h}$ prior to performance. One aim of this thesis is to look at alternative options to optimise athletic performance, that would be practical to implement within elite sport, subsequently the use of prior exercise, hormonal priming and limb IPC will be discussed for use as pre-competition strategies. The following section will highlight mechanisms involved in prior exercise, hormonal priming; 
the mechanisms associated with limb IPC and sport performance will be outlined after clinical mechanisms of IPC have been discussed.

\subsubsection{Hormone response to exercise}

Aside from priming mechanisms discussed above, the effects of androgen $T$ on strength and muscle adaptations to exercise have been well reported (Sinha-Hikim et al. 2004). Testosterone is a steroid hormone produced from cholesterol via a series of conversions catalysed by specific enzymes (Vingren et al. 2012) and T is considered a key anabolic hormone with multiple physiological functions in the human body. The primary production site of $\mathrm{T}$ is the Leydig cells, found in the testes, which explains approximately 10-fold higher circulating concentrations in men (Vingren et al. 2012). Whereas $T$ is produced in small quantities in the ovaries and the zona reticulans of the adrenal cortex in women (Maroulis \& Triantafillidis 2006), conversion of androgens is another source of $\mathrm{T}$ in females (Vingren et al. 2012). The signal for $\mathrm{T}$ production and release originates in the hypothalamus which is innervated by the central nervous system (CNS) and provides a direct link between the nervous and endocrine systems (Kim 2007). Within the hypothalamus, gonadotrophin releasing hormone $(\mathrm{GnRH})$ is produced and secreted, this stimulates the production and release of lutenising hormone (LH) and follicle-stimulating hormone (FSH) from the gonadotrophs (Kim 2007). In the gonads, LH stimulates T production in the Leydig cells of men and theca cells of women; LH binds to a G-protein coupled membrane receptor which activates cyclic adenosine monophosphate-dependent protein kinases (protein kinase A) which stimulates the rate limiting step in T synthesis (Miller 1988). In women, $\mathrm{T}$ is further processed to estradiol in the granulosa cells adjacent to the theca cells, FSH stimulates production of pregnenolone in the granulosa cells and steroid binding protein production in the liver (Vingren et al. 2012). The signal cascade from FSH is similar to that induced by LH in the theca and Leydig cells; pregnenolone can leave the granulosa cells for the theca cells where it can be further processed to T (Vingren et al. 2012). Initiation of the hypothalamic pituitary gonadal (HPG) axis, leading to $T$ release, is caused either by direct nervous stimulation of the hypothalamus by the CNS or by reduced feedback inhibition on the hypothalamus by $\mathrm{T}$ (Vingren et al. 2012). Testosterone induces negative feedback on the hypothalamus to reduce GnRH release and the gonadotrophs in the anterior pituitary to reduce the release of LH and FSH in response to GnRH; the signal for increased T response with exercise is controlled at the hypothalamus (Vingren et al. 2012).

Testosterone is important for sports performance as it can promote increases in voluntary exercise (Beaven et al. 2008; Crewther et al. 2014); athlete T levels have been positively correlated to selfselected workloads (Goto et al. 2007) whilst greater $\mathrm{T}$ responses to competition have been associated with higher motivation to win (Hellhammer et al. 1985), thus fluctuations in T could be a major factor determining competition performance. For example, within elite populations, 
Crewther et al. (2012) found salivary T concentrations to be highly correlated with both squatting $(\mathrm{r}=0.92)$ and sprint times $(\mathrm{r}=0.87)$ in elite strength trained athletes, but not so much in their less strong counterparts.

Research suggests total $\mathrm{T}$ and free $\mathrm{T}$ are elevated following heavy resistance exercise in men. There appears to be a relative intensity and volume threshold that must be reached to induce a $T$ response; it is well known that brief, intense physical exertion increases the circulating $\mathrm{T}$ levels (Cumming et al. 1987). Acute exercise increases T linearly once a specific threshold intensity is reached, with peak concentrations usually occurring at the end of exercise. From a physiological perspective, Lin et al. (2001) observed that lactate in-vitro dose-dependently increased the production of $\mathrm{T}$ by acting on rat Leydig cells; intensity and volume therefore need to be sufficient to induce this response. Further research by Ratamess et al. (2005) showed that six sets, but not one set of 10 repetitions of squats significantly increased total $\mathrm{T}$ post exercise, suggested to be the result of a larger increase in lactate and high metabolic demand. Beaven et al. (2008) also identified greatest increase in $\mathrm{T}(13.1 \pm 19.9 \%)$ following three sets of five repetitions performed at $85 \%$ of $1 \mathrm{RM}$ and also following four sets of ten repetitions performed at $70 \% 1 \mathrm{RM}$ (increase $11.3 \pm 19.7 \%$ ) whereas three sets of five repetitions performed at $40 \% 1 \mathrm{RM}$ caused a $3.5 \pm 29.7 \%$ decrease in $\mathrm{T}$. These studies highlight the importance of both intensity and volume to stimulate an increase in $\mathrm{T}$.

Alongside T, C is also a biomarker of particular interest in sport (Cook et al. 2014). Cortisol, the principal glucocorticoid in humans, or otherwise known as the major stress hormone, targets an array of both peripheral and central processes (Lupien et al. 2007); the regulation of stress-induced as well as basal C secretion is thus essential for the maintenance of homeostasis (McEwen 2000; Tsigos \& Chrousos 2002). Cortisol is released from the adrenal cortex in response to stress during exercise (Kon et al. 2012), and promotes catabolism via decreased protein synthesis and increased protein degradation (Kraemer \& Ratamess 2005). When triggered outside of circadian rhythms, $\mathrm{C}$ release is specific to stress (Herman et al. 2005). The key target of the various direct and indirect pathways is the paraventricular nucleus (PVN) of the hypothalamus (Herman et al. 2005), upon perception of acute stress, cells within the PVN release corticotropin releasing hormone which travels through the infundibulum to the pituitary gland where it stimulates secretion of adrenocorticotropic hormone (ACTH) into the bloodstream (Dedovic et al. 2009). ACTH binds to receptors in the adrenal cortex, that stimulate secretion of $\mathrm{C}$ into the blood stream. The majority of cells in the human body have receptors for $\mathrm{C}$, thus having a broad variety of effects including metabolic, cardiovascular and immune responses (Buckingham 2009; McEwen 2000). Glucocorticoids are released from the adrenal cortex in response to the stress of exercise, of which $\mathrm{C}$ accounts for approximately 95\% (Kraemer \& Ratamess 2005). Due to the major role C plays in 
tissue remodeling, acute and chronic changes in response to resistance exercise have been examined (Kraemer \& Ratamess 2005).

In sport, $\mathrm{C}$ is often used as a marker of both physiological and psychological stress (Teo et al. 2011), prolonged elevation of $\mathrm{C}$ has been shown to exhibit an inhibitive effect on the neuromuscular system (Teo et al. 2011). For example, Tafet et al. (2001) reported a negative correlation between physical performance and prolonged elevated salivary $\mathrm{C}$ levels. However, the rapid hormone response to maximal exercise promotes the mobilisation of energy reserves during physical activity by stimulating gluconeogenesis, promoting lipolysis and increasing protein catabolism (Viru \& Viru 2004). Previous research has highlighted that the magnitude of the acute endocrine response is influenced by the intensity and volume of the resistance exercise with high volume, moderate to high intensity protocols eliciting greater $\mathrm{C}$ responses (Kraemer \& Ratamess 2005). For example, Kim (2007) reported significant increases in $\mathrm{C}$ in females in response to three sets of ten repetitions at $80 \% 1 \mathrm{RM}$. Thus, the $\mathrm{C}$ response to resistance exercise appears to be dependent on the stress and metabolic requirements of the exercise (Goto et al. 2005).

The impact of exercise on hormone response in females is not so clear, with an increase in free $T$ following resistance exercise found in some studies (Nindl et al. 2001; Copeland et al. 2002; Häkkinen \& Pakarinen 1995; Vingren et al. 2009) but not all studies (Linnamo et al. 2005; Kraemer et al. 1993; Marx et al. 2001), along with inconsistent evidence of $\mathrm{C}$ response to exercise. For example, Linnamo et al. (2005) demonstrated no changes in total $\mathrm{T}$ following five sets of ten repetitions for three exercises using three different loading conditions. Whereas in men, the same maximal loading condition lead to a significant increase in total $\mathrm{T}$ (Linnamo et al. 2005). Kraemer et al. (1991) measured serum T levels before, during, and after resistance exercise and found that circulating $\mathrm{T}$ is significantly increased in males and was not changed in females throughout the duration of the exercise. This is supported by Weiss et al. (1983) investigating sex differences in androgen response to heavy resistance exercise. The T levels of 20 men and 20 women were measured following weightlifting; $\mathrm{T}$ increased significantly in men by $21.6 \%$ but the increase was not significant in women (16.7\%), with men having $43 \%$ higher resting levels than women. The biological mechanisms for a potential exercise-induced increase in $\mathrm{T}$ might be different in females as they do not have Leydig cells and Kvorning et al. (2007) have shown that the Leydig cells are likely involved in acute resistance exercise-induced increase in $\mathrm{T}$ in men. In comparison Edwards \& Neal (2009) identified an increase in T from pre-to post competition in female athletes from intercollegiate sport, reporting oral contraceptive users and non-users, whether analysed by absolute change in $\mathrm{T}$ level or by percent change from before-competition value, increased in response to competition. This effect is in accordance with reports for college-age women rugby players (Bateup et al. 2002). However, it has also been acknowledged that a simulated team 
handball match did not increase saliva T (Filaire \& Lac 2000), suggesting (by comparison to the results reported above) that a simulated competition lacking meaning to participants may be relatively ineffective in elevating $\mathrm{T}$ level in comparison to a competition fixture. Furthermore, Cook \& Beaven (2013) suggested elite female athletes have higher baseline T levels than nonelite females and secondly individual $\mathrm{T}$ concentrations demonstrated strong relationships with selfselected workloads during training, similar to results reported in male participants. Therefore, this provides evidence elite females may exhibit similar $\mathrm{T}$ response to exercise. The variation in previous research may be explained by previous research not considering the effect of competition, athlete level and menstrual cycle or oral contraceptives (e.g. Dabbs \& de La Rue 1991; Sellers et al. 2007).

The nature of inter-hormonal dynamics in females and response to exercise requires more research. Pronounced levels of estradiol levels occur over the course of the menstrual cycle with low levels in the early follicular phase, which peak shortly before or during ovulation and slowly decrease throughout the luteal phase (Kirschbaum et al. 1999). In women, T serves as a precursor for estradiol, the primary (pre-ovulation) peak and secondary (luteal) peak of $\mathrm{T}$ are followed by similar peaks of estradiol (Celec et al. 2009) (Figure 2.1). However, research has suggested when adding day of menstrual cycle into a regression model to test if stability changed as a function of menstrual cycle phase, the effect of menstrual cycle on stability was non-significant for $\mathrm{T}$ and $\mathrm{C}$, for instance $\mathrm{C}$ was found to be highly stable in both men $(\mathrm{r}=0.93)$ and women $(\mathrm{r}=0.73)$ (Liening et al. 2010). Basal C levels before stimulation or immediately after awakening in the morning did not show cyclic variation when previously measured in the follicular versus luteal phase, in agreement with previous reports (Fujimoto et al. 1986; Kanaley et al. 1992). Research has shown men, and women in the luteal phase had the largest salivary free $\mathrm{C}$ responses, which were clearly enhanced when compared to women in the follicular phase in response to a psychosocial test (Kirschbaum et al. 1999). In contrast to the biologically active free hormone fraction, total blood C levels masked these differences with similar levels between the groups, this may suggest female adrenal cortex may be more sensitive to ACTH (Roelfsema et al. 1993; Horrocks et al. 1990) with no apparent change of net $C$ secretion in follicular compared to luteal phases (Kirschbaum et al. 1999). The capacity of the female adrenal cortex to synthesise and secrete $\mathrm{C}$ in response to maximal stimulation is similar to that of men. However, when in the luteal phase, women may display higher circulating salivary free C levels after ACTH stimulation (Kirschbaum et al. 1999). Following morning and evening measurement of salivary hormone concentrations across one menstrual cycle (luteinising hormone marked mid-cycle), it was concluded that the menstrual cycles were statistically significant but smaller than daily cycles or individual differences (Dabbs \& La Rue 1991). 
The use of oral contraceptives should also be considered when examining women's baseline T, C, and response to training and competition. Oral contraceptives can decrease the blood or salivary levels of $\mathrm{T}$ and other androgens in healthy women (Coenen et al. 1996; Graham et al. 2007; Wiegratz et al. 2003; Zimmerman et al. 2014). Oral contraceptives are low doses of exogenouslyadministered synthetic hormones for the naturally occurring levels of estrogen and progesterone that would otherwise vary systematically as part of a normal menstrual cycle (Edwards \& Neal 2009). Reports highlight oral contraceptive users have significantly lower saliva $T$ values than non-users which may result from decreased ovarian secretion of $\mathrm{T}$ owing to oral contraceptive inhibition of hypophyseal secretion of FSH and LH. Moreover, oral contraceptive-related decreases in free $\mathrm{T}$ levels are much greater (61\%) than those observed for total $\mathrm{T}(31 \%)$ and likely due to oral contraceptive-related increase in steroid binding proteins (e.g. sex-hormone binding globulin) (Coenen et al. 1996; Zimmerman et al. 2014), thereby reducing the bioactive free steroid, along with suppression of ovarian and adrenal androgen synthesis (Zimmerman et al. 2014). Schultheiss et al. (2003) determined the impact of menstrual cycle phase and oral contraceptive use on steroid hormone levels in saliva; $\mathrm{T}$ levels were significantly lower in women using oral contraceptives, matching other research that found the same effect (Edwards \& Neal 2009; Anders \& Watson 2006; Wirth \& Schultheiss 2007), however, Schultheiss et al. (2003) found that T levels did not significantly differ across the menstrual cycle for both normally-cycling women and women using oral contraceptives. It is worth noting, oral contraceptive use did not account for any significant differences in levels of $\mathrm{C}$ or progesterone. 


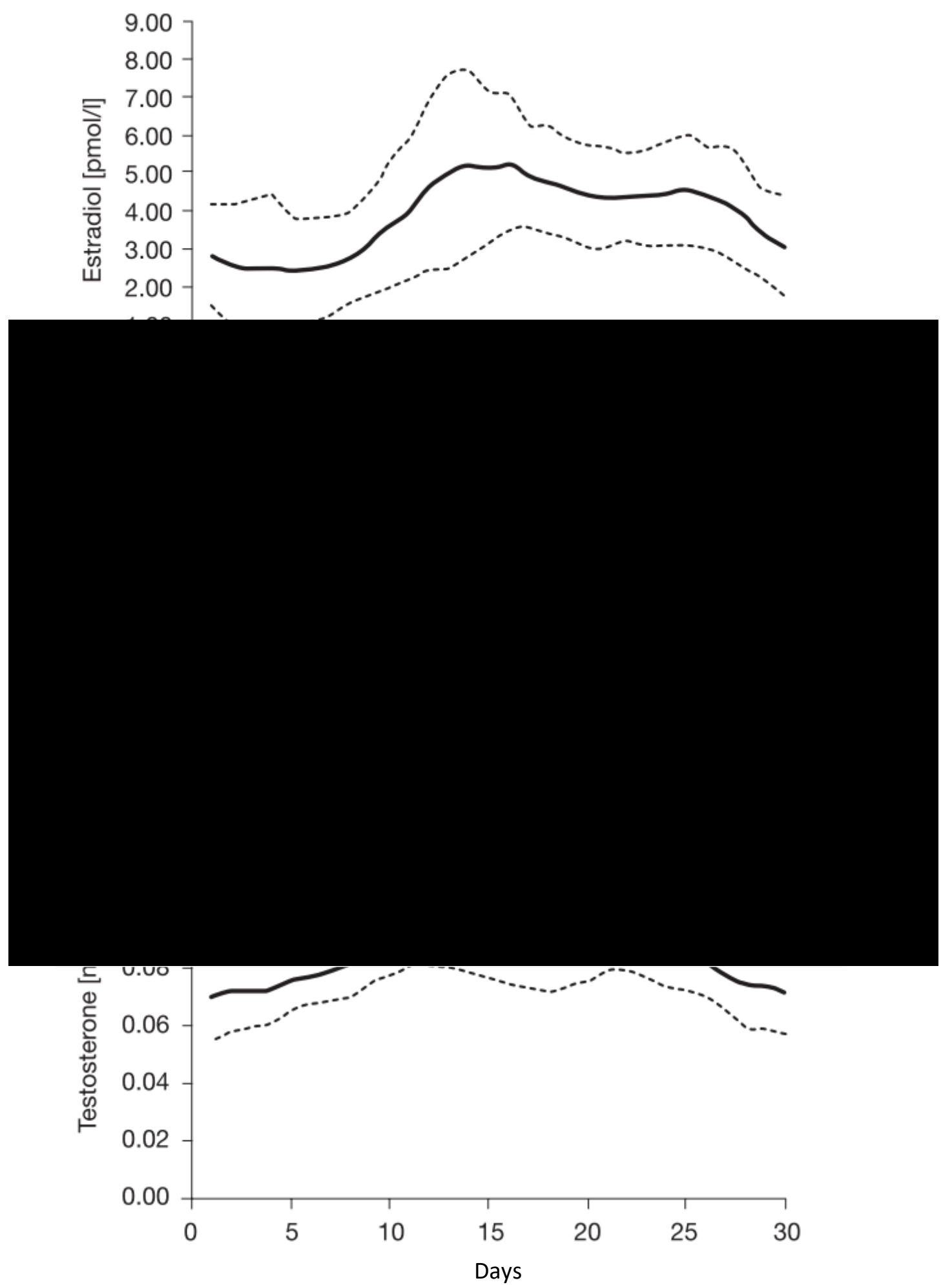

Figure 2.1 Dynamic changes of salivary estradiol, progesterone and testosterone during one menstrual cycle in young healthy women (Adapted from Celec et al. 2009) 
However, research has suggested that women using oral contraceptives were found to repeatedly show blunted free $\mathrm{C}$ responses to psychosocial or physical stress (Kirschbaum et al. 1996). It is unknown whether lower free $\mathrm{C}$ responses reflect a hypo-reactive HPA axis or if oral contraceptive users secrete similar amounts of $\mathrm{C}$, but due to increased production of corticosteroid-binding globulin induced by oral contraceptive medication, the biologically active free $\mathrm{C}$ fraction is largely reduced (Kirschbaum et al. 1999). Whereas contrasting research has reported oral contraceptive use was associated with lower levels of T, but did not affect $C$ levels (Liening et al. 2010); for instance, when adding oral contraceptive use into a regression model to test if stability changed as a function of oral contraceptive use, the effect of oral contraceptives on stability was nonsignificant for both $\mathrm{T}$ and progesterone (Liening et al. 2010). In contrast, oral contraceptive use was found to significantly affect $\mathrm{C}(\mathrm{p}=0.01)$, however, women using oral contraceptives had a higher stability coefficient $(\mathrm{r}=0.81, \mathrm{p}<0.01)$ than normally-cycling women $(\mathrm{r}=0.75, \mathrm{p}<0.01)$ (Liening et al. 2010).

Although oral contraceptive users are responsive to sports competitions (Edwards \& Casto 2013), some research has demonstrated oral contraceptive users display lower absolute $\mathrm{T}$ levels in response to training and competition (Crewther et al. 2015). However, Edwards \& Neal (2009) demonstrated although oral contraceptive users had significantly lower saliva T levels than nonusers before and after competition, both users and non-users showed virtually the same increase in saliva $\mathrm{T}$ over the course of a competition suggesting reporting the change in $\mathrm{T}$ response may be beneficial for females within future research. Furthermore it has been evidenced that individual differences in levels of salivary $\mathrm{C}$ (Liening et al. 2010) and $\mathrm{T}$ tend to be relatively stable (Edwards \& Kurlander 2010).

\subsubsection{Circadian rhythmicity and morning exercise}

Both $\mathrm{T}$ and $\mathrm{C}$ basal levels exhibit circadian rhythms, which are elevated in the morning with a nadir in the late afternoon (Kanaley et al. 2001; Kraemer et al. 2001). Accordingly, circadian rhythmicity of specific physiological responses such as endocrine release have been proposed to modulate characteristics deemed key to sports performance (Teo et al. 2011). Circadian rhythms are endogenous biorhythms that persist on cyclic periods of approximately $24 \mathrm{~h}$ and have been shown to influence a wide array of physiological systems (Hadley \& Levine, 2006). In humans, the primary circadian pacemaker is the suprachiasmatic nucleus (SCN) which is located within the hypothalamus (Teo et al. 2011). The SCN receives direct input regarding the solar cycle from the retina (Hastings \& Herzog 2004). With this information, the SCN coordinates daily biological rhythms (i.e. hormone secretion, temperature fluctuations, neural activation) in line with the solar time and sleep wake cycle (Buijs et al. 2003; Waterhouse et al. 2005). Many physiological 
functions associated with athletic performance have been shown to follow a specific circadian rhythm (Winget et al. 1985).

The basal concentrations of $\mathrm{T}$ and $\mathrm{C}$ are regulated by the hypothalamus, as previously discussed, by pulsatile secretion that defines both ultradian and circadian rhythmicity (Beaven et al. 2010). There are many underlying factors contributing to circadian rhythms in physical performance. These factors can stem from both internal (physiological) and external (environmental) changes that occur throughout the day (Teo et al. 2011). Reilly \& Waterhouse (2009) identified 3 major determinants in the contribution towards a circadian rhythm in sporting performance. The first determinant takes into consideration external (environmental) influences that are usually uncontrollable. The second determinant involves internal (physiological) influences and lastly lifestyle (psycho-biological) influences affecting timing preferential in activities and sleeping patterns. Regarding the internal physiological influences, Buśko \& Opaszowski (2005) have shown rapid elevation in T, unlikely to be explained by the 'typical' hormonal cascade of HPG axis activation, which involves the release of secretagogines from the arcuate nucleus and anterior pituitary gland (Beaven et al. 2010). The pulsatile release and rapid changes of endogenous steroids as a result of an exercise stimulus, overlay the circadian rhythm and characterise an ultradian rhythm that has the effect of exposing tissues to large fluctuations of hormones (Young et al. 2004; Veldhuis et al. 2008). Acknowledging the circadian rhythmicity of $\mathrm{T}$ and $\mathrm{C}$, there is opportunity to influence competition performance as resistance exercise has shown to increase $\mathrm{T}$ and pre-workout $\mathrm{T}$ levels have correlated with the snatch $(\mathrm{r}=0.70)$ and clean and jerk $(\mathrm{r}=0.62)$ 1RM lifts in males. The benefits of resting T concentrations and exercise performance during tests of power and strength have been established (Cardinale \& Stone 2006); T contributes to neuromuscular performance and has been shown to produce rapid changes in intracellular calcium $\left(\mathrm{Ca}^{2+}\right)$ in muscle cells (Estrada et al. 2003) which can regulate membrane potential and muscle contractility (Crewther \& Cook 2010). Considering the potential role of $\mathrm{T}$ in mediating athletic performance, it could be advantageous to attempt to offset the circadian decline in $\mathrm{T}$ as athletes maybe requested to perform in both the morning and afternoon (Cook et al. 2014). 
Table 2.1 The influence of early morning physical activity on afternoon performance

\begin{tabular}{|c|c|c|c|c|}
\hline (Author \& Date) & AM exercise & $\begin{array}{l}\text { Time between AM \& PM } \\
\text { measures (h) }\end{array}$ & PM performance & Outcome \\
\hline (Cook et al. 2014) & $\begin{array}{l}\text { 1.Running } 5 \times 40 \mathrm{~m} \text { sprints } \\
\text { 2.Weights } 3 \mathrm{RM} \text { bench } \\
\text { press \& squat } \\
\text { 3.Passive rest }\end{array}$ & 6 & $\begin{array}{l}\text { 3RM bench \& squat, } 40 \mathrm{~m} \\
\text { sprint, CMJ }\end{array}$ & $\begin{array}{l}\text { Weights improved PM } \\
\text { performance \& reduced } \\
\text { decline in T AM to PM }\end{array}$ \\
\hline (Ekstrand et al. 2012) & $\begin{array}{l}\text { 1.Back squat to failure and } \\
4 \text { reps per set power clean } \\
\text { 2.Passive rest }\end{array}$ & $4-6$ & $\begin{array}{l}\text { Backward overhead shot and } \\
\text { VJ }\end{array}$ & $\begin{array}{l}\text { Increased throws but not jump } \\
\text { performance }\end{array}$ \\
\hline (McGowan et al. 2016) & $\begin{array}{l}1.1200 \mathrm{~m} \text { swim } \\
2.1200 \mathrm{~m} \text { swim \& land } \\
\text { resistance exercise } \\
\text { 3.Passive rest }\end{array}$ & 6 & 100m swimming TT & $\begin{array}{l}1.6 \% \text { and } 1.7 \% \text { improvement } \\
\text { swim performance from AM } \\
\text { exercise }\end{array}$ \\
\hline (Russell et al. 2015) & $\begin{array}{l}\text { 1.Bench press } 5 \times 10 \text { at } \\
75 \% 1 \mathrm{RM} \\
\text { 2.Cycling } 6 \times 6 \mathrm{~s} \text { sprint } \\
\text { 3.Running } 6 \times 40 \mathrm{~m} \text { sprints } \\
\text { 4.Passive rest }\end{array}$ & 5 & $\begin{array}{l}\text { CMJ, reaction time, repeated } \\
\text { sprint ability }(6 \times 40 \mathrm{~m})\end{array}$ & $\begin{array}{l}\text { PM sprint improved \& } \\
\text { reduced decline in T AM to } \\
\text { PM after AM weights \& } \\
\text { running. PM jump height } \\
\text { increased after cycling \& } \\
\text { running. }\end{array}$ \\
\hline
\end{tabular}

AM - morning; PM - afternoon; CMJ - countermovement jump; VJ - vertical jump; RM - repetition maximum; T - testosterone 
Of practical importance, early morning physical activity may influence subsequent activities performed later in the day (Ekstrand et al. 2012). Considering competition is frequently held in the late afternoon when $\mathrm{T}$ has declined, athletes may be susceptible to changes in their physical performance as a function of time, as morning training may influence afternoon performance (Cook et al. 2014) (Table 2.1). Previous research by Ekstrand et al. (2012) demonstrated that a morning resistance session improved throwing performance in well trained shot putters when measured in the afternoon. This has been supported by McGowan et al. (2016), finding morning exercise improved afternoon running and swimming performance, respectively. However, hormone concentrations were not measured within this previous research, whereas additional research completed by Cook et al. (2014) reported morning strength training was associated with improvements in CMJ PPO, $40 \mathrm{~m}$ sprint times, 3RM bench and squat performance performed $6 \mathrm{~h}$ later in rugby union players in alignment with research in this area. However, a strength of this research highlighted morning strength training offset the circadian decline in T. Further research by Russell et al. (2015) measured the hormone response to the effect of morning exercise on the circadian decline in $\mathrm{T}$, whilst also acknowledging the practical considerations associated with precompetition practices and the limited application of the methods of morning exercise examined previously. Therefore, four interventions were assessed; control, weights, cycling and running (Table 2.1). After $5 \mathrm{~h}$ rest, $3 \mathrm{CMJ}$ and repeated sprint ability were assessed. Afternoon sprint performance improved after weights and running, with greater $\mathrm{T}$ concentrations in the afternoon following weights $(+17 \pm 18 \%, \mathrm{p}=0.002)$. Despite acknowledging practical application, five sets of ten repetitions is still a high volume for some athletes and sports such as weightlifting, where $\mathrm{T}$ may impact performance, therefore alternative strategies are well sought after, evidencing the practical opportunity to implement vascular occlusion.

\subsubsection{Fatigue and recovery}

Sport performance and training can result in short term fatigue due to induced physiological adaptations, mechanical and biochemical changes (Bishop et al. 2008) which can negatively affect subsequent performance or result in injury (Bishop et al. 2008) or illness (Cunniffe et al. 2011). Fatigue has previously been defined as sensations of tiredness and associated decrements in muscular performance and function (Kay et al. 2001; Green 1997; Millet et al. 2002; Pinniger et al. 2000). As fatigue is considered an inevitable and negative consequence of physical activity (Kay et al. 2001; Pinniger et al. 2000) there has been much research into its effect on exercise performance (Millet et al. 2003; Millet et al. 2000; Gibson et al. 2001; Lepers et al. 2000; Kay et al. 2000). It has been hypothesized that fatigue has two origins; (i) in the central fatigue hypothesis, the muscles are believed capable of greater output but the CNS acts as a protective mechanism to prevent excessive damage to the muscles (Taylor et al. 2000) which denotes that the CNS fails to drive the motor neurons adequately (Gandevia 2001). (ii) Peripheral fatigue, a state whereby the 
muscles homeostasis has been perturbed, either metabolically through tissue damage, induced alterations in muscle homeostasis (e.g. depletion of creatine phosphate or accumulation of inorganic phosphate) or alterations in excitability due to potassium $\left(\mathrm{K}^{+}\right)$efflux from the cell (Allen et al. 2008), to the point that the muscle is biochemically or mechanically incapable of responding as effectively as it does when rested (Macintosh \& Rassier 2002). Physiological stress induced by intense exercise symptomatically manifests post exercise as a decrease in NMF, increased muscle soreness, stiffness and swelling (Bishop et al. 2008). Athletes completing exercise whilst experiencing these symptoms will likely perform below their potential (Howatson \& van Someren 2008). Along with these perturbations, physical activity can affect the musculature, resulting in EIMD. EIMD is a phenomenon that occurs as a result of unaccustomed, eccentric exercise inducing disruption of the sarcomeres (Brown \& Hill 1991; Fridén et al. 1983), disruption of the cytoskeletal elements that are involved in force transmission (Koh \& Escobedo 2004), damage to the muscle cell membrane (Lovering \& De Deyne 2004; McNeil \& Khakee 1992), impaired excitation-contraction coupling (Warren et al. 1993), loss of $\mathrm{Ca}^{2+}$ homeostasis and a subsequent loss of force production (McCully \& Faulkner 1985). Exercise induced alterations in muscle homeostasis include hydrogen ion $\left(\mathrm{H}^{+}\right)$accumulation, $\mathrm{K}^{+}$, depletion of high energy phosphates (ATP and creatine phosphate) and glycogen (Suzuki et al. 2002; Steele \& Duke 2003). Alongside these symptoms, an increase in intramuscular proteins in the blood is present; this is the result of increased intracellular $\mathrm{Ca}^{2+}$ derived from extracellular sources leading to myofibrillar damage in skeletal muscle (Duncan 1987; Gissel \& Clausen 2001). The influx of $\mathrm{Ca}^{2+}$ into the cytosol initiates a cascade of events that further damage the cell by causing alteration to the cytoskeleton, sarcoplasmic reticulum, mitochondria and myofilaments (Gissel \& Clausen 2001; Byrd 1992). The $\mathrm{Ca}^{2+}$ mediated responses appear to be manifested by the activation of proteolytic and lipolytic pathways that lead to degradation of the cell membrane and sarcolemma, cell infiltration and activation, production of reactive oxygen species (ROS) (Jones et al. 1986) and ultimately regeneration of fibres after several days (Fridén et al. 1983). The loss of membrane integrity allows for leakage of intramuscular proteins such as $\mathrm{CK}$, which can be seen in the blood for many days post exercise (Warren et al. 1999). The presence of the muscle proteins and enzymes in the blood is a commonly reported indicator of muscle damage (Ehlers et al. 2002; Howatson \& van Someren 2008). The response of CK to exercise has received research attention, as strenuous exercise that damages skeletal muscle cell structure results in an increase in blood CK activity.

An understanding of the physiological, neuromuscular and perceptual challenges in sport is required. Accordingly, various methods are used in applied practice to indicate the presence of EIMD and the efficacy of recovery interventions (Ferguson et al. 2014; Bishop et al. 2008). Although valid as independent measures, there is rationale for measuring a set of markers especially as EIMD is a phenomenon encompassing various physiological processes (Damas et al. 
2016). The use of biomarkers providing combination of biological and performance tests offers a more thorough understanding of the physiological responses to exercise, therefore, $\mathrm{CK}$ and hormone response are frequently monitored to provide indirect evidence of muscle damage (Bessa et al. 2016). Specifically within team sports, alongside $C K$, and $T$ and $C$ hormonal milieu, NMF and perception of muscle soreness are indirect markers used to assess EIMD, fatigue and recovery with disruptions previously observed post-match lasting at least $48 \mathrm{~h}$ (Cunniffe et al. 2010; Shearer et al. 2015; West et al. 2014; Russell et al. 2015; Twist et al. 2012).

Creatine Kinase is an enzyme that catalyses the transfer of phosphate from phosphocreatine ( $\mathrm{PCr}$ ) to adenosine diphosphate (ADP) (Koch et al. 2014). As discussed, EIMD can result in the leakage of CK out of the cell and into the blood stream as well as other biochemical markers including lactate dehydrogenase, leukocyte levels, Tumor necrosis factor-alpha, iL-6 and CRP as reported by Bessa et al. (2016). Creatine Kinase provides a more practical marker in the field to measure as venous blood samples are not required, offering a safer, quicker and less invasive option. Previous research reviewing biomarkers of exercise intensity and recovery highlighted CK levels increased $(\mathrm{p}<0.01)$ from 12 to $24 \mathrm{~h}$ after exercise and returned to pre-exercise values within $48 \mathrm{~h}$ (Bessa et al. 2016). This is supported by numerous studies showing increases in CK following team sport matches (Jones et al. 2014; Russell et al. 2015; Russell et al. 2016; Shearer et al. 2015; Twist et al. 2012). For example, Thorpe \& Sunderland (2012) identified 84\% increase in CK from pre-to post-match with similar results reported by McLellan et al. (2010) (peak CK values $24 \mathrm{~h}$ post-match) and Russell et al. (2015) confirming these results. Limitations should be acknowledged regarding CK measurement presented in the literature (e.g. variance across individuals due to ethnicity, gender or age; Bessa et al. 2016), however, CK measured in plasma has been reported to be a reliable indirect marker of EIMD (Koch et al. 2014).

Hormonal disruption is also commonly used to assess physiological response and readiness to train; $\mathrm{T}$ and $\mathrm{C}$ have been identified as reliable markers of the endocrine response to competitive sports performance (Clarkson et al. 1992; Cunniffe et al. 2009; McLellan et al. 2010). Testosterone is the primary anabolic marker for protein signalling (Suzuki 2004) and muscle glycogen synthesis (Salvador et al. 2003). Cortisol is considered an important stress hormone and acts antagonistically with $\mathrm{T}$ to mediate catabolic activity, increasing protein degradation and decreasing protein synthesis in muscle cells (Hoffman et al. 2002). It is possible the various hormonal and muscle enzyme measures may assist in assessing the immediate response and time course of recovery after competition (McLellan et al. 2010). The use of salivary $\mathrm{T}$ and $\mathrm{C}$ assay measures provides a relatively simple, non-invasive procedure that provides a valid and reliable indication of plasmaunbound C (Cadore et al. 2008; Vining et al. 1983) and plasma-free T (Wang et al. 1981). In 
response to a single soccer match, an anticipatory rise in $\mathrm{C}$ has been reported with increases during the match; equivocal findings for T have been observed (Thorpe \& Sunderland 2012).

Decreases in free $\mathrm{T}$ up to $48 \mathrm{~h}$ may occur after acute exercise, it has been suggested that catecholamine may be responsible for the prolonged inhibitory effect of stress on $\mathrm{T}$ biosynthesis (Tanaka et al. 1986). However, decreases may be attributable to increased utilisation of $\mathrm{T}$ during exercise to preserve protein tissue or via a decreased rate of production because of inhibitory mechanisms (Kraemer et al. 1991; Kraemer et al. 2004; Kraemer et al. 2007). Other potential mediators explaining observed decreases in $\mathrm{T}$ with exercise involves the role of stress hormones such as $\mathrm{C}$. It is thought that elevations in $\mathrm{C}$ with exercise may interfere with $\mathrm{T}$ production, either acutely during exercise or chronically as a result of training (Kraemer et al. 2004; Kraemer et al. 2007). Changes in hormone $\mathrm{T} / \mathrm{C}$ ratio have been suggested as a possible indictor of physiological strain (Fry \& Kraemer 1997). Alterations in $\mathrm{T}$ and $\mathrm{C}$ are thought to represent a disturbance in overall anabolic/catabolic balance of the athlete (Hoogeveen \& Zonderland 1996) and are known to affect the recovery state and duration of recovery after exercise (Fry et al. 1991). A change in this balance is dependent on intensity and duration of exercise (Hoogeveen \& Zonderland 1996); a decrease in free $\mathrm{T} / \mathrm{C}$ ratio of $30 \%$ or more in plasma has been shown to indicate temporary incomplete recovery from intensive training in elite speed skaters (Banfi et al. 1993).

Functional tests involving completion of maximal jumps which evaluate PPO and fatigue remain the primary tools to assess recovery in the field (Bessa et al. 2016). PPO is used as a measure to assess NMF, frequently assessed by a CMJ performed on a force platform, as previously established as a criterion method to determine peak mechanical power (Owen et al. 2014). Research suggests the CMJ to be one of the most used tests for monitoring NMF in individual and team sports (Claudino et al. 2016) with numerous researchers finding CMJ performance to be an objective marker of fatigue (Balsalobre-Fernańdez et al. 2014; Jiménez-Reyes \& González-Badillo 2011; Cormie et al. 2009; Coutts et al. 2007); a recent meta-analysis (Claudino et al. 2016) reported the use of average CMJ height to monitor neuromuscular status. Several studies, while reporting an initial decline immediately after the stimulus, observed a short-term recovery in NMF following a second decrease in performance between 24 and $48 \mathrm{~h}$ post, with the mechanisms behind this pattern potentially linked to the time frames associated with the onset of recovery from metabolic disturbance and muscle damage, respectively (Kuitunen et al. 2004). A likely explanation for the initial decrease might be a reduced functioning of the contractile mechanisms of the muscle fibre (Skurvydas et al. 2007) in the presence of metabolites produced during exercise $\left(\mathrm{H}^{+}, \mathrm{ADP}\right.$, Phosphate [Pi]). 
A practical, non-invasive method to assess fatigue and recovery is the use of subjective measures including perception of recovery and muscle soreness. Due to the practicality, research is validating and assessing reliability of these measures. For instance, Impellizzeri \& Maffiuletti (2007) have shown perceived lower body muscle soreness scale (7-point scale) to be a valid and reliable measure of lower limb soreness whilst Laurent et al. (2011) observed participants accurately assessed perceived levels of recovery using the perceived recovery scale (11-point scale) indicated by correspondence with negative and positive changes in total sprint time relative to a prior session. Further research is required; however, the results indicate a subjective approach may be an effective means for assessing recovery.

To date, multiple studies have investigated NMF, hormonal, biochemical and perceptual postmatch fatigue. Twist et al. (2012) monitored the perceptual, NMF and biochemical responses to a rugby league match. The results identified perceived muscle soreness and fatigue both increased relative to baseline (pre-match) on both day one and day two post-match (24 and $48 \mathrm{~h}$ ). CK was also elevated day one and day two whereas flight time of CMJ was reduced in all players immediately post-match and on day one but was recovered by day two suggesting recovery between measured variables i.e. tissue damage from CK but impaired central drive affecting CMJ was affected by disruption of $\mathrm{Ca}^{2+}$ homeostasis and impaired excitation-contraction coupling (Twist et al. 2012). Results are similar when identifying response to a single maximal speed training session; Johnston et al. (2015) reported blood lactate increased immediately post session with CMJ PPO decreasing at the same time point. Muscle soreness increased immediately, $2 \mathrm{~h}$ and $24 \mathrm{~h}$ post training session along with $\mathrm{CK}$ also increasing and remaining elevated at the same time points, whereas $\mathrm{T}$ and $\mathrm{C}$ both decreased at $2 \mathrm{~h}$ post but returned to baseline by $24 \mathrm{~h}$ after the session terminated. The combination of markers to assess fatigue and recovery are being increasingly applied in practice due the variance in physiological responses to exercise and are monitored in response to competition and training to ensure sufficient recovery to optimise subsequent performance. These markers also help determine the efficacy of recovery interventions by allowing acknowledgement of the area of performance and physiology that is impacted whether this is NMF or clearance of biochemical or hormonal markers.

Recovery strategy efficacy may also be determined by monitoring stress-induced hormone reactivity, providing information on individual recovery and readiness to train or compete. Changes in hormone $\mathrm{T} / \mathrm{C}$ ratio have been suggested as a possible indictor of physiological strain (Fry \& Kraemer 1997). Adlercreutz et al. (1986) proposed the use of free T/C ratio as a diagnostic or preventative test to detect overtraining as it gives an indication of the anabolic/catabolic balance in response to training and competition, which are known to affect the recovery state and duration of recovery after exercise (Fry et al. 1991). Crewther \& Cook (2012) investigated post-match 
interventions and subsequent hormonal responses to a physical stress test and game performance to measure stress-induced hormone reactivity. Positive feedback to a video of match performance was associated with significantly $(\mathrm{p}<0.01)$ greater free $\mathrm{T}(36$ to $42 \%)$ responses to a physical stress test when compared to negative feedback provided ( 16 to -3\% free T). Positive feedback was also associated with higher (28 to 51\%) pre-game $\mathrm{T}$ and superior game performance. This is supported by Gonzalez-Bono et al. (1999) reporting T levels affected the contribution individuals made to team competition performance. Literature indicated $\mathrm{T}$ regulates adaptive physiology and physical performance (Crewther et al. 2011). This induces rapid bioavailability and effects on the CNS, such that human behaviour (motivation) towards physical performance may be modified through acute perturbations (Crewther et al. 2011). Extending the above literature, research has highlighted a larger $\mathrm{T}$ response and lower $\mathrm{C}$ response to a mid-week stress test was found before winning (vs losing) (Crewther et al. 2017). Therefore, responses to a mid-week stress test dicate what happens in performance after, how recovered players are from previous matches and following performance. Therefore, $\mathrm{T}$ could provide an important mediator of athlete recovery, moreover $\mathrm{T}$ response to a physical stressor may be indicative of recovery and performance following an association between $\mathrm{T}$ response to a mid-week exercise stressor (three sets of power cleans, three sets of back squat and three sets of bench press at 40,60 and 70\% 1RM) and subsequent winning or losing (Crewther et al. 2017). Thus, monitoring of T responsiveness to a physical 'stress test' could provide additional information on competitive readiness, future resilience and potentially a marker of recovery intervention success.

Keeping in mind EIMD, hormonal disruption and potentially reduced NMF, the post-competition recovery period is an integral component of the management of players/athletes (West et al. 2014). Insufficient recovery, with ensuing fatigue, could potentially lead to poor or inconsistent performance (Barnett 2006) magnified during the competitive season due to accumulative effects from both competition and training (Maso 2004). It is evident in the sport setting there is need for suitable methods to enhance the rate of recovery after fatiguing exercise (Pinar et al. 2012). Recovery of muscle function is primarily a matter of reversing the major cause of fatigue or damage (Bishop et al. 2008). Since the causes of fatigue may be many and varied, there are numerous approaches to restoring homeostasis in the muscle cell (Bishop et al. 2008). For the purpose of this review, as reported by Bishop et al. (2008), recovery is the initial period between successive training sessions or competition with the potential to improve subsequent performance. Recovery has become a key facet of weekly preparation and training regimen (Kellmann 2010) to ensure continued optimal performance in successive training and matches (Taylor et al. 2014). Consequently, recovery strategies are defined as the implementation of a technique or combination of techniques to accelerate the time to achieve full recovery and potentially reduced the risk of injury (Dupont et al. 2010). These strategies include, but are not limited to cold water immersion, 
contrast water therapy, active recovery, neuromuscular electrical stimulation, compression garments and massage. For reviews of these strategies refer to Nédélec et al. (2013) and Bishop et al. (2008) as this is outside the scope of this review.

Several mechanisms are discussed within the literature regarding the underlying purpose of using recovery strategies; strategies aim to reduce acute inflammation from muscle damage and enhance its rate of removal are reported to be a priority in soccer (Nédélec et al. 2013). These strategies are believed to improve the rate of recovery through the manipulation of blood flow, for example, cold water immersion is thought to reduce blood flow to the legs, inducing a redirection of blood flow from the periphery and thereby improve venous return and cardiac efficiency (Vaile et al. 2011), which is assumed to improve $\mathrm{O}_{2}$ and nutrient delivery, and therefore ATP, PCr and glycogen resynthesis (Byrne et al. 2004). One efficient mechanism of recovery enhancement at muscle level may be the augmented removal of exercise metabolites by improving microcirculation (Pinar et al. 2012). However, previous recovery strategies are inconsistent in success of increasing rate of recovery and also are not practical for situations such as away games where equipment, time and space are required. Practical recovery strategies are sought after and/or additional strategies due to inconsistencies in the literature to date. Consequently, an emerging strategy termed IPC or vascular occlusion will be discussed as an alternative option.

\subsection{CLINICAL BACKGROUND AND DEFINITIONS}

Ischemia refers to the restriction in blood supply to a tissue, causing a shortage of $\mathrm{O}_{2}$ required for metabolism (Kalogeris et al 2012). Prolonged ischemia may lead to cellular dysfunction, apoptosis and cell death, which might be amplified by reperfusion (the return of blood supply to tissue after ischemia), resulting in IRI after restoration of blood flow (Jean-St-Michel et al. 2011).

Vascular occlusion originated in the medical literature, in which this strategy referred to intervals of sub-lethal ischemia-reperfusion applied to the heart to provide protection against lethal IRI that occur during myocardial infarction, subsequently reducing tissue damage (Hausenloy \& Yellon 2008). Within a clinical setting it is a strategy utilised to exploit the body's endogenous protective capabilities against the injury incurred by IRI (Hausenloy \& Yellon 2008). In its original concept, it described intra-myocardial protection, which could be relayed from the myocardium served by one coronary artery to another (Hausenloy \& Yellon 2008). The brief episodes of ischemia were shown to slow the rate of ATP depletion during subsequent ischemic episodes and therefore it was thought multiple brief ischemic episodes (strategy termed IPC) might protect the heart from a subsequent sustained ischemic insult (Murry et al. 1990). Specifically, IPC refers to the phenomenon whereby 3-4 brief periods of ischemia, followed by tissue reperfusion, confers 
subsequent protection against the magnitude of tissue injury following ischemia (Thijssen et al. 2016). IPC was first described in a study by Murry et al. (1986), in which the hearts of anaesthetised dogs were preconditioned with 4 cycles of 5 min occlusions of the circumflex artery, each separated by 5 min of reperfusion (Sharma et al. 2015). This was followed by a sustained 40 min occlusion and 4 days of reperfusion. The extent of myocardial infarction in the preconditioned hearts was found to be dramatically reduced to a mere $25 \%$ of that seen in the control hearts which did not receive preconditioning (Murry et al. 1986).

However, the clinical utility of IPC was limited by the invasive approach that needed to achieve ischemia in the target organ before a predictable injury (Jean-St-Michel et al. 2011). An alternative, non-invasive strategy applied the cardio-protection remote from the heart, an approach referred to as remote ischemic preconditioning (RIPC); this idea was first conceived by Przyklenk et al. (1993) and referred to this phenomenon as 'preconditioning at a distance'. This was followed by studies showing that preconditioning of the heart could be achieved by applying brief episodes of ischemia and reperfusion to a remote organ such as the kidney or other abdominal visceral organs (Gho et al. 1996). Birnbaum et al. (1997) made the critical observation that RIPC could also be applied to the limbs. In this investigation, brief cycles of blood flow restriction combined with brief cycles of blood flow restriction with electrical stimulation to the gastrocnemius muscle were applied to induce demand ischemia (Birnbaum et al. 1997). When applied prior to sustained coronary artery occlusion and reperfusion, this intervention reduced infarct size by more than $65 \%$. Kharbanda et al. (2001) were the first to demonstrate that the application of an RIPC stimulus without the need for electrical stimulation reduced the extent of myocardial infarction in-vivo in pigs. This gave rise to a non-invasive method of protecting the heart against lethal IRI that could be applied in a clinical setting. Oxman et al. (1997) demonstrated application of a tourniquet to the hind-limb to induce ischemia, had the ability to reduce reperfusion arrhythmias in a rat heart following a sustained ischemia insult. Through medical development, a non-invasive strategy is now practiced in patients, involving the application of the cardio protection to an organ or tissue remote from the heart (Przyklenk et al. 1993).

The concept of remote organ protection has been extended beyond that of protecting the heart to providing inter-organ protection against IRI including skeletal muscle (Hausenloy \& Yellon 2008). This has been achieved by applying inflated blood pressure cuffs to the upper or lower limbs (Hausenloy \& Yellon 2008). Kharbanda et al. (2002) and Loukogeorgakis et al. (2005) pioneered the use of this approach in human volunteers and in patients. In the original study, transient ischemia and reperfusion of the arm was induced by inflating a blood pressure cuff placed on the upper arm to $200 \mathrm{mmHg}$ for $5 \mathrm{~min}$ and deflating the cuff for $5 \mathrm{~min}$; a cycle which was repeated 3 times (Kharbanda et al. 2002). This RIPC stimulus was able to attenuate the endothelial 
dysfunction in the contralateral arm arising from a 20-min episode of sustained cuff inflation. The same experimental model has been used to reproduce the concept of delayed RIPC, in which the pre-conditioning upper limb ischemia confers an improvement in endothelial function in the contralateral arm 24-48 h later (Loukogeorgakis et al. 2005).

\subsubsection{Clinical mechanisms}

Mechanisms of IPC have been well established; IPC appears to begin with ligand binding Gprotein coupled receptors on the surface of the cardiomyocytes (Sharma et al. 2015). These ligands include adenosine, bradykinin, opioids, angiotensin and endocannabinoids (Sharma et al. 2015). The binding of the cardiomyocyte surface receptors to the respective ligands initiates signalling pathways, which eventually lead to changes in the myocardium, rendering it resistant to IRI (Hausenloy \& Yellon 2008; Sharma et al. 2012), specifically, reports suggest adenosine can increase $\mathrm{O}_{2}$ delivery by dilation of the resistance vessels and decreased inatrophy, which decreases myocardial $\mathrm{O}_{2}$ demand (Eisen et al. 2004). Expression of signalling molecules leads to activation of mediators that transmit the protective signal in the cytosol (Thijssen et al. 2016). Ultimately, the signalling pathways converge at the mitochondria, the most important effector of protection induced by IPC (Thijssen et al. 2016), with the opening of the mitochondrial permeability transition pore being a crucial step in this process (Davidson et al. 2006). Eisen et al. (2004) also described receptor independent triggers, identifying them as nitric oxide (NO), free radicals and $\mathrm{Ca}^{2+}$. The effect of these receptors may be further explained by mediators of IPC; the protective effect of IPC is thought to be due to intracellular mediators of which 2 have been identified; ATP sensitive potassium channels $\left(\mathrm{K}_{\mathrm{ATP}}\right)$ and protein kinase C (PKC) (Eisen et al. 2004). Inner mitochondrial membrane $\mathrm{K}_{\mathrm{ATP}}$ contribute to the protective effects of IPC against prolonged ischemia (Ardehali \& O'Rourke 2005). Further research supports this, in which the myocardial sarcolemmal and the mitochondria $\mathrm{K}_{\mathrm{ATP}}$ have been proposed to be critical activators or mediators in the cardioprotective mechanism of IPC (Yellon \& Downey 2003). The current theory suggests that the ligand-receptor binding at the cell surface activates signal transduction pathways, which terminate at the mitochondria and result in the opening of the mitochondrial $\mathrm{K}_{\mathrm{ATP}}$ channels (Hausenloy \& Yellon 2008). This leads to the generation of mitochondrial ROS that facilitate cardioprotection by either activating pro-survival kinases (Yellon \& Downey 2003) or inhibiting the opening of mitochondrial permeability transition pore. In contrast, Gürke et al. (2000) assessed the possible involvement of $\mathrm{K}_{\mathrm{ATP}}$ channels and adenosine receptors in IPC of skeletal muscle in a rat model. It was identified that either IPC or the $\mathrm{K}_{\mathrm{ATP}}$ channel opener cromakalim significantly improved post-ischemic muscle function. The protective effect of cromakalim was not seen when the $\mathrm{K}_{\mathrm{ATP}}$ channel blocker glibenclamide was added, however this did not block IPC induced protection. Therefore, the results show significant improvements in post-ischemic skeletal muscle function after IPC or cromakalim pre-treatment, but do not support a role of $\mathrm{K}_{\mathrm{ATP}}$ channels or 
adenosine receptors in IPC of skeletal muscle. However, alternative reports have suggested modulation of $\mathrm{K}_{\mathrm{ATP}}$ channels reduced ATP depletion, preserved intracellular $\mathrm{pH}$ and enhanced recovery of ATP and phosphocreatinine levels during reperfusion (Takaoka et al. 1999). These observations are supported (e.g. Pang et al. 1997) and suggest $\mathrm{K}_{\text {AтP }}$ channels serve as an end effector in adenosine pathways (Tapuria et al. 2008).

Alternative mechanisms and impacts of IPC on physiological responses have been investigated, for example Murray et al. (1990) identified preconditioning reduced starting levels of ATP by $29 \%$, but an episode of IPC to the heart resulted in a decrease in adenine nucleotide pool at 0 min of ischemia in comparison to the control $(20.4 \pm 0.4$ vs $29.2 \pm 0.3)$. This identified that IPC caused a slowing in the initial rate of adenine nucleotide degradation during sustained ischemia, with preconditioning resulting in depletion of nearly $50 \%$ of myocardial glycogen reserves. The biochemical analyses from the study revealed energy metabolism during ischemia was slowed by preconditioning; resulting in reduced accumulation of metabolic products and preservation of ATP stores (Murray et al., 1990). A reduced metabolic demand could slow the rate of high-energy phosphate utilisation, consequently preserving ATP stores and reducing the rate of anaerobic glycolysis (Murray et al., 1990). An accumulation of glucose-1-phosphate, glucose-6-phosphate and lactate were shown to be reduced by preconditioning, indicating a reduction in rate of anaerobic glycolysis and high energy phosphate depletion and a limitation of catabolite accumulation (Murray et al., 1990). The study reported this to be the most likely explanation for slower metabolic activity; suggesting if glycolytic rate were limited by glycogen depletion it would have been expected that ATP depletion would have occurred more rapidly in the IPC trial (Murray et al., 1990). It was therefore proposed that IPC reduces myocardial energy demand during ischemia (Murry et al., 1990).

Within clinical literature, there are reports of an early and delayed phase of IPC (Figure 2.2); the delayed or late phase has been known as the 'second window' (Fryer et al. 2002). Baxter et al. (1997) first characterised the time-course of the late phase of IPC and demonstrated that it appears 24,48 , and 72 hours following brief periods of preconditioning ischemia. IPC studies have shown that adenosine mediates both the early and the late phases of preconditioning via different endorgan receptors (Fryer et al. 2002). In the heart adenosine acts via the A1 receptor; adenosine receptors mediate anti-adrenergic effects indirectly by reducing cyclic adenosine monophosphate (cAMP) levels. The A1 receptor-mediated effects involve activation of PKC, tyrosine kinases (Dana et al. 2000), heat shock protein, and mitogen-activated protein kinase, whereas kinases modulate end effector mitochondrial $\mathrm{K}_{\mathrm{ATP}}$ channels (Tapuria et al. 2008). Activation of these receptors and their effects are due to endothelium dependent and independent mechanisms, indirectly through release of $\mathrm{NO}$ and through direct relaxation of vascular smooth muscles. NO 
modulates microvascular perfusion through its vasodilatory effect (Jaeschke et al. 1996) and through its anti-inflammatory actions, including inhibition of stellate cell activation (Rockey \& Chung 1995), neutrophil adhesion (Lefer \& Lefer 1996), and platelet aggregation (Tapuria et al. 2008). The early phase of preconditioning, mediated by endogenous NO, is through generation of cAMP, as shown by Lochner et al. (2002) in the myocardium and subsequently inhibition of cAMP levels as well as reduction in energy demands. The late phase is protein synthesis dependent and is through activation of protein kinase $\mathrm{C}$ and transcription of inducible NO (Nandagopal et al. 2001). Therefore, this biphasic adaptation has been attributed to distinct cellular mechanisms (Franz et al. 2017); early effects are thought to be related to an altered ionic channel permeability or post-translational modification of proteins, while late effects could be induced by an altered gene expression or immune response (Murphy et al. 2010; Konstantinov et al. 2004).

However, the underlying mechanisms of RIPC are not clear, the pathway through which the protective signal is transmitted from a remote organ to organs targeted by RIPC is still being investigated (Sharma et al. 2014). The literature has proposed several theories and mechanisms that may be involved; reports suggest the peripheral stimulus can be chemical, mechanical or electrical and involves activation of peripheral sensory nerves (Heusch et al. 2015), with the signal transfer to the heart and other organs associated with neuronal and humoral communications (Heusch et al. 2015) (Figure 2.3). Further studies have investigated whether endogenous substances such as adenosine, opiods (e.g. Patel et al. 2002) and endocannabinoids (e.g. Hajrasouliha et al. 2008) are released from the remote organ during IPC and are carried to the heart in the blood stream where they have been proposed to activate intracellular pathways of cardioprotection (Hausenloy \& Yellon 2008). Two alternative mechanisms have also been proposed; (i) the influence of the neural pathway and RIPC generating an endogenous substance e.g. adenosine, which activates a local afferent neural pathway stimulating an efferent neural pathway; the effects of adenosine include vasodilation's, inhibition of leukocyte adhesion, neutrophil and platelet function (Bouma et al. 1997) and free radical production (Tapuria et al. 2008) and (ii) a systemic response which may suppress inflammation and apoptosis (Pope et al. 2013).

The hypothesis for a neural pathway was further developed with the proposition that endogenous substances such as adenosine and bradykinin released by the remote preconditioned organ, stimulated afferent nerve fibres (Hausenloy \& Yellon 2008). Regarding a systemic response, studies have investigated the effect of RIPC on the myocardial gene transcription profile (Konstantinov et al. 2005) and the inflammatory response (Peralta et al. 2001) and have discovered that the inflammatory response is suppressed and a favourable profile of gene transcription appears to be achieved that is both anti-inflammatory and anti-apoptotic (Hausenloy \& Yellon 2008). 
This concept, based on assumption that a tissue with some history of ischemic conditions eventually becomes more resistant to ischemia and its potential deleterious effects (Kocman et al. 2015; Lott et al. 1996; Ovize et al. 2013) attracted many researchers in sports medicine and exercise science. The demonstration of the favourable effects of IPC and RIPC on endothelial function, skeletal muscle and the heart raised the possibility that in addition to the benefits identified in a clinical setting, IPC could also be applicable within sport. Consequently, a reasonable number of experimental researchers have attempted to demonstrate beneficial effects of IPC on athletic performance as discussed in the following sections. 


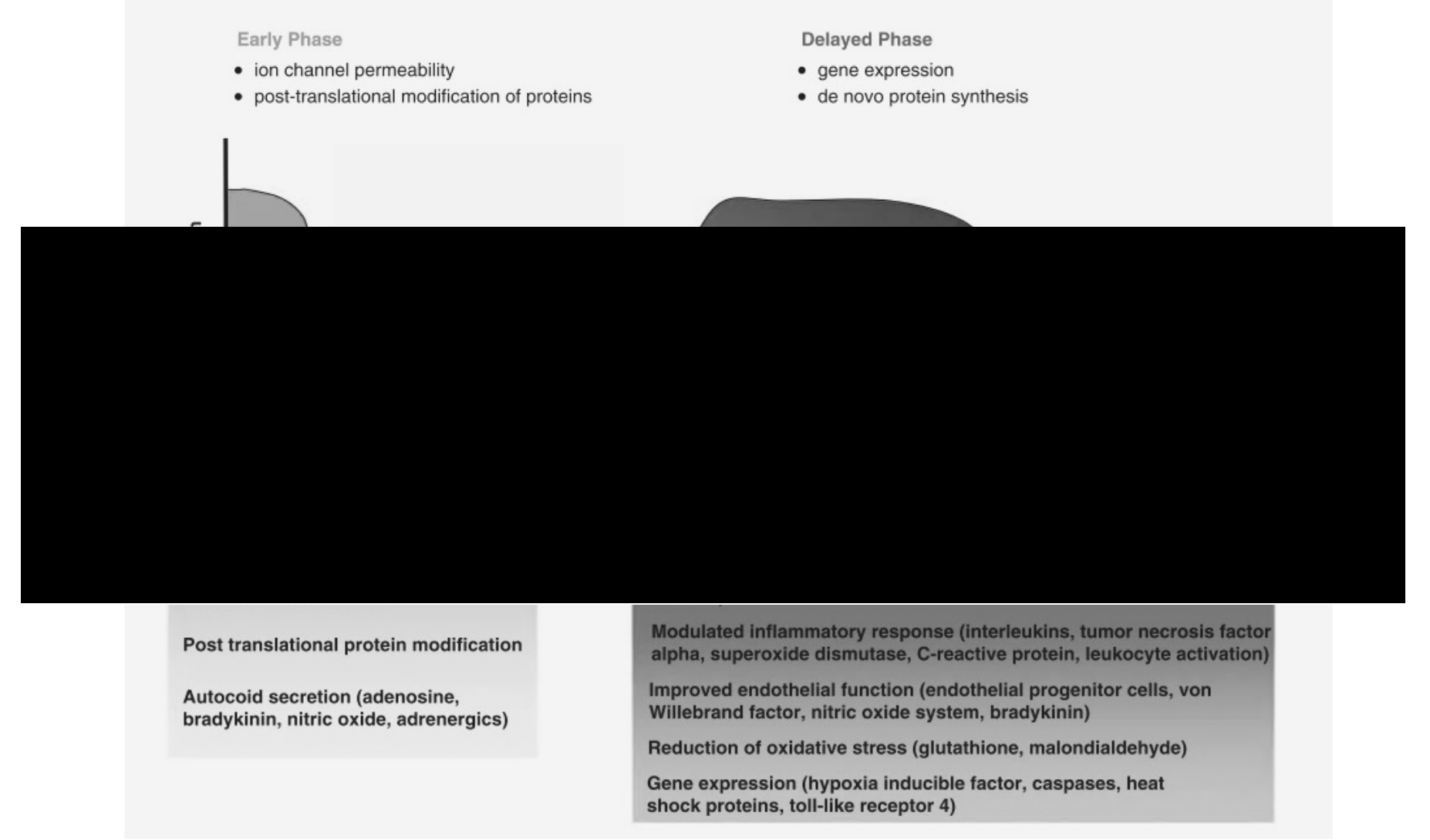

Figure 2.2 Mechanisms of the early and delayed (late) phase of ischemic preconditioning (adapted from Koch et al. 2014) 


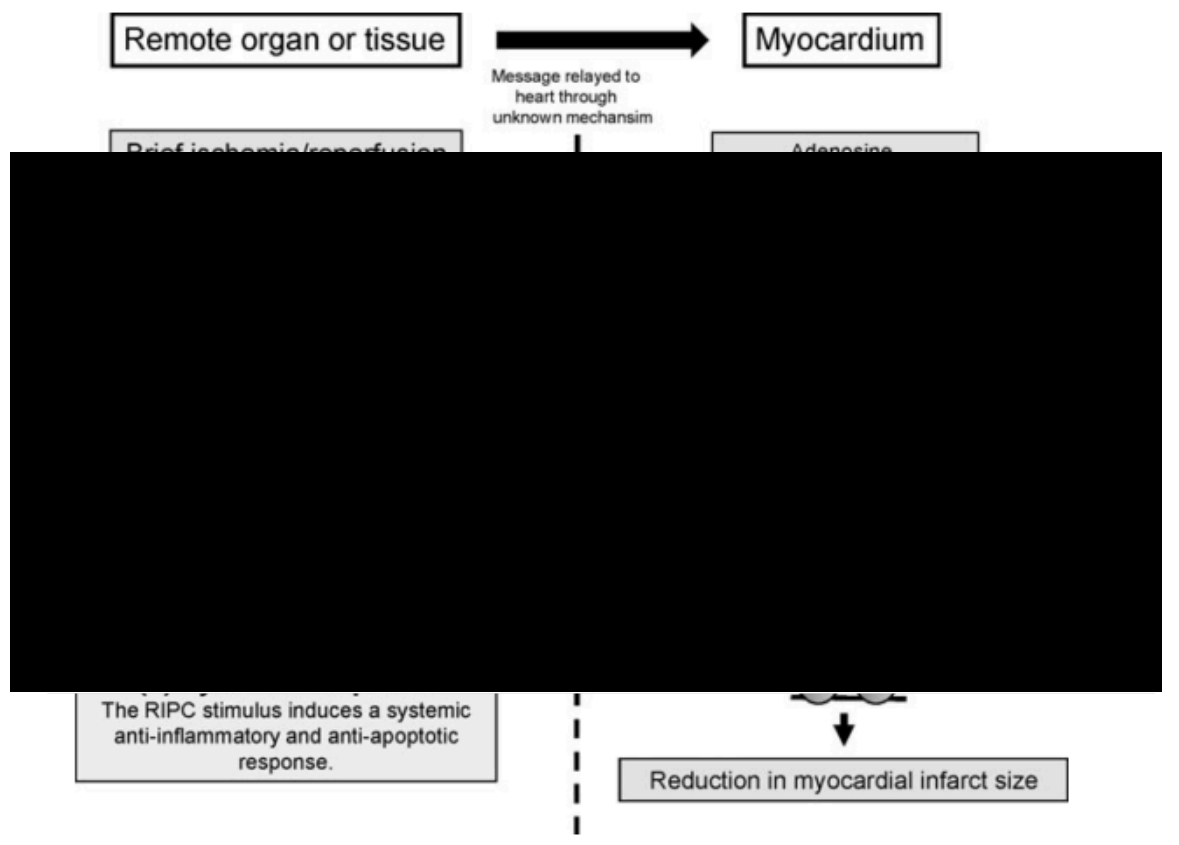

Figure 2.3 Potential mechanistic pathways underlying remote ischemic preconditioning (extracted from Hausenloy \& Yellon 2008) 


\subsubsection{Introduction to IPC and vascular occlusion in sport performance}

The discovery of IPC as a potential tool for improving skeletal muscle performance was first investigated by Libonati et al. (1998); they associated IPC with higher force generation. It is important at this stage to note the term IPC and comparison to RIPC and limb IPC. IPC referred in the clinical literature to an invasive method of pre-conditioning the heart, the definition of RIPC is related to an intervention that does not directly affect the same part/system of the body where it is applied (Przyklenk et al. 1993). In this respect, some studies suggested a humoral hypothesis postulating that an ischemic event leads to the production of substances that could reach another tissue (Marocolo et al. 2016). Previous research has argued that RIPC would not likely be relevant in the majority of sports, if applied to the legs for example, and subsequent sports performed involved the lower extremities; the application would not be 'remote' and therefore, it is the concept of IPC applied to the limb that may have potential to improve exercise performance via both local effects (i.e. to the limb) and remote effects (via cardiovascular or nervous system); this has been referred to as limb IPC to distinguish from the concept of using RIPC to target the remote organ alone. Therefore, for the purpose of this thesis, limb IPC will be terminology used to describe the application of this strategy to the limbs, within a sports performance environment, where cycles of ischemia and reperfusion are used as a pre-conditioning strategy and vascular occlusion will describe the application of cuffs and blood flow restriction during exercise or for use as a recovery strategy.

Within the context of sports performance, limb IPC or vascular occlusion is achieved via application of an external pressure over the proximal portion of the upper or lower extremities (Pope et al. 2013), achieved using a tourniquet, pressurised cuff or elastic band. Generally, the limb IPC protocol involves four cycles of five min occlusion, followed by five min reperfusion period (Thijssen et al. 2016). As this method is relatively easy to conduct and non-invasive, limb IPC may have various advantages in athletic settings (Horiuchi 2017). The aim of external pressures is to maintain partial arterial inflow to the muscle while occluding venous return (Pope et al. 2013) resulting in the pooling of venous blood around the exercising muscle when occlusion is combined with exercise (Fahs et al. 2012) or create cycles of ischemia and reperfusion when applied during passive rest.

Figure 2.4 summarises the suggested mechanisms for the use of vascular occlusion combined with exercise, limb IPC or for use as a recovery strategy. The proposed theory behind the use of vascular occlusion combined with exercise (previously used as a training modality), suggests improved athletic performance due to a reduction in intramuscular $\mathrm{O}_{2}$ delivery (Tanimoto et al. 2005) and decreased venous clearance of metabolites (Inagaki et al. 2011). This can lead to exaggerated levels of metabolic acidosis, which consequently may increase hormone response or increased 
fibre type recruitment (Yasuda et al. 2011), which may improve muscle contraction efficiency through increased muscle force and contractility (Lawson \& Downey 1993) along with an increased efficiency of excitation contraction coupling (Pang et al. 1995).

The mechanisms relating to limb IPC or vascular occlusion during passive rest, have been speculated to include improvements in the vasculature, optimising blood flow to working tissues, improvements in oxygenation during exhaustive exercise (De Groot et al. 2010) and improvement in mitochondrial metabolism (Andreas et al. 2011). The majority of the literature attributes improved performance to the release of adenosine, which provides a protective effect (Goto et al. 1995) as well as causing vasodilation, which subsequently increases blood supply during exercise (De Groot et al. 2010). This can improve performance due to increased metabolic waste removal (Vaile et al. 2011) and repletion of ATP (Connolly et al. 2003). In vivo studies have shown that limb IPC or vascular occlusion can lead to opening of mitochondrial $\mathrm{K}_{\mathrm{ATP}}$ and uncoupling of oxidative phosphorylation (Fryer et al. 2000). It has been speculated that this allows for a faster uptake of aceyl-CoA and thus maintains lactate accumulation at a metabolically acceptable level (Jean-St-Michel et al. 2011). As included in Figure 2.4 the late phase has been associated with ROS, FMD and $\mathrm{NO}$ and consequently increased $\mathrm{O}_{2}$ delivery and extraction along with attenuation of endothelial function to improve aerobic contribution to performance.

To date, limited research has investigated the mechanisms associated with use of vascular occlusion as a recovery modality, however from speculation, it has been suggested that performance is improved consequence of increased blood flow associated with elevated adenosine levels (Liu et al. 1994) and activation of $K_{\text {ATP }}$ channels (De Groot et al. 2010) which increase vasodilation and increase removal of $\mathrm{H}^{+}$. It has also been hypothesized that ischemia may reduce the inflammatory response, decreasing intramuscular pressure and subsequently decreasing pain and muscle soreness associated with EIMD (Figure 2.4).

\subsubsection{Summary}

Despite an increasing number of studies examining the effects of limb IPC and vascular occlusion on exercise performance, preliminary conclusions regarding the beneficial effects are difficult to establish due to inconsistent methods used among studies including protocols, exercise modes, exercise durations, timings and populations. The next sections will highlight current literature which has investigated the use of limb IPC and vascular occlusion for use as a pre-competition or recovery strategy and the suggested mechanisms influencing performance. 


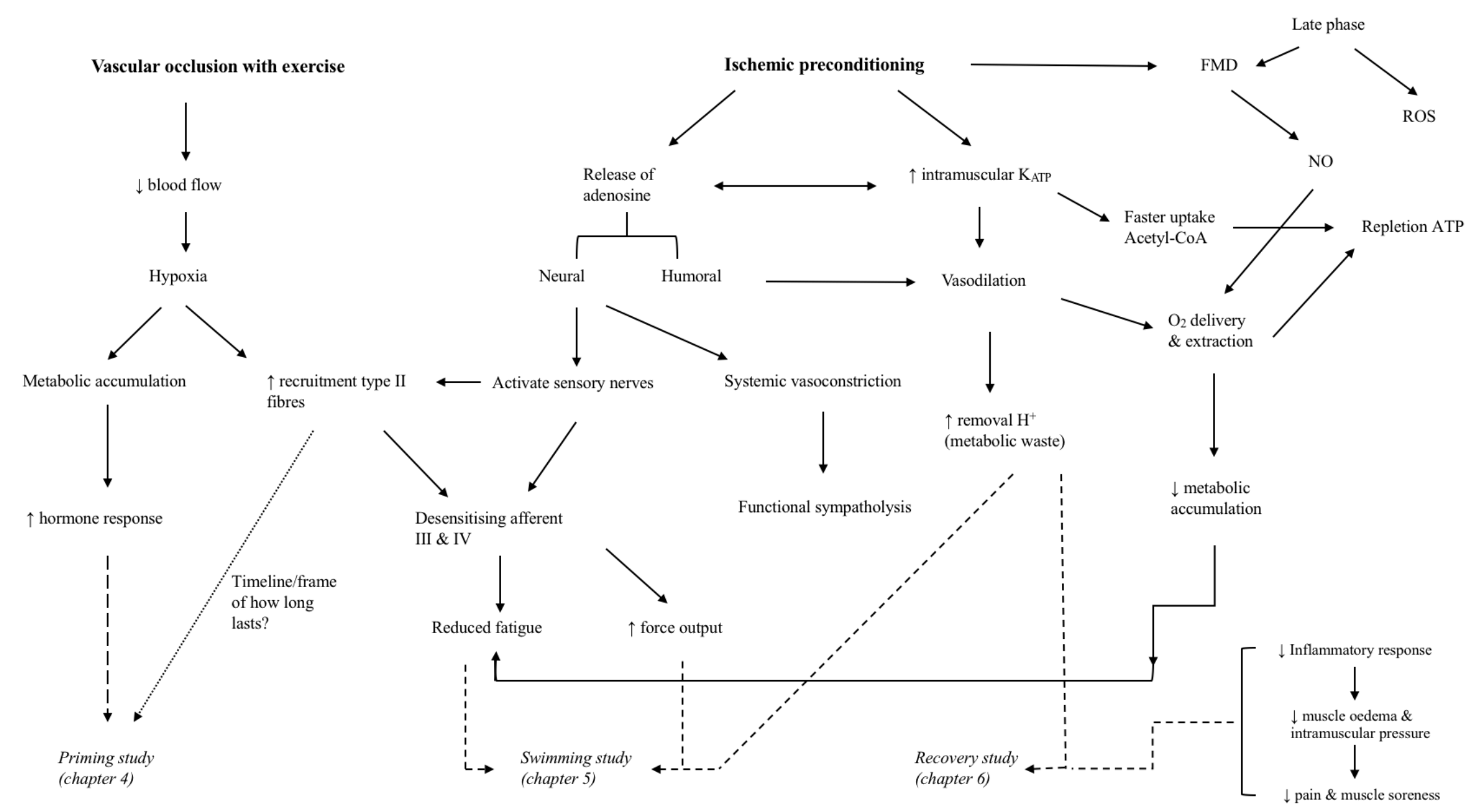

Figure 2.4 The mechanisms associated with each experimental chapter and application of vascular occlusion or limb IPC as pre-competition and recovery strategies 


\subsection{PRE-COMPETITON: THE USE OF VASCULAR OCCLUSION AND LIMB IPC}

On the day of competition there is an opportunity to enhance performance using pre-competition strategies. As previously discussed, these strategies include appropriate warm up protocols, passive heat maintenance, post activation potentiation, priming and more recently vascular occlusion and limb IPC (Kilduff et al. 2013). Within the constraints of athletic performance, the benefits of limb IPC have been observed in time to exhaustion (e.g. De Groot et al. 2010; Crisafulli et al. 2011), anaerobic performance tests (e.g. Bailey et al. 2012; Jean-St-Michel et al. 2011; Lisbôa et al. 2017) and muscle activation (e.g. Yasuda et al. 2014; Teixeira et al. 2017). Although there is some disparity in findings between the effects on athletic performance, it can be postulated that vascular occlusion and limb IPC may provide an ergogenic aid for competition preparation.

\subsubsection{Vascular occlusion with resistance exercise}

Vascular occlusion in combination with exercise has been demonstrated to result in numerous positive training adaptations and acute physiological responses. Within the literature, the effects of vascular occlusion with various exercise modes (e.g. cycling, walking, resistance exercise) have been examined, however for the purpose of this review the focus will remain on vascular occlusion with resistance exercise.

The ability to increase muscle strength and hypertrophy when combined with vascular occlusion is different from the traditional paradigm, which suggests lifting higher intensities to increase such characteristics (Pope et al. 2013). For example, The American College of Sports Medicine recommends lifting weights of at least $70 \% 1 \mathrm{RM}$ to achieve muscular hypertrophy, through increased mechanical work, which increases amino acid transport into cells and enhances the synthesis of contractile proteins (Schoenfeld 2010). However, many studies have shown muscle hypertrophy to occur with training as low as $20 \% 1 \mathrm{RM}$ when combined with vascular occlusion (Abe et al. 2005; Abe et al. 2005; Fujita et al. 2008; Luebbers et al. 2014). Therefore low-load resistance exercise (20-50\% of $1 \mathrm{RM})$, combined with vascular occlusion, has been proposed as an alternative or addition to heavy resistance exercise. Of particular benefit, Yasuda et al. (2011) reported that vascular occlusion training does not require a long recovery time between training sessions (Abe et al. 2005; Yasuda et al. 2009) owing to the very low mechanical stress and minimal muscle damage (Abe et al. 2005; Fujita et al. 2008) therefore allowing for repeated training sessions within a short time frame or preparation for competition performance.

There has been conflicting evidence regarding the benefits and outcomes of this strategy, reports have shown no effects of vascular occlusion on hypertrophy or tension properties (Kubo et al. 
2006; Barcelos et al. 2015), along with no additional benefit compared to traditional resistance exercise (e.g. Kim et al. 2012). However, different applications for the use of training with vascular occlusion within an athletes training program are emerging. For example, during a busy, high volume schedule it can allow for continued resistance exercise training that achieves the same response as high intensity resistance exercise traditionally required, that cannot ordinarily be complete.

Lowery et al. (2014) completed a protocol consisting of individuals using vascular occlusion either for the first 4 weeks or second 4 weeks of an 8 -week resistance exercise program. Both groups, regardless of order, increased muscle thickness significantly from baseline to week 4 (6.9\% and $8.6 \%, \mathrm{p}<0.01)$ and from weeks 4 to week $8(4.1 \%$ and $4.0 \%, \mathrm{p}<0.01)$ respectively. This identified that similar hypertrophy gains were possible from low intensity resistance exercise with vascular occlusion compared to high intensity resistance exercise without vascular occlusion. This can allow for lighter loads to be utilised in the return from injury, but still achieve the same benefit for athletic performance.

An alternative strategy has examined combining high intensity resistance exercise and low intensity resistance exercise with vascular occlusion into one training program. Luebbers et al. (2014) investigated the effect of a 7-week vascular occlusion protocol on measures of muscular strength. Sixty-two American Football Players were divided into four groups, one of which completed supplemental lifting with vascular occlusion. Within this group greater 1RM squat performance $(\mathrm{p}<0.000)$ was achieved compared to the traditional program with supplemental lifts without vascular occlusion ( $\mathrm{p}=0.025$ ) and the traditional high intensity resistance exercise program $(\mathrm{p}=0.009)$. This study was a modification of that implemented by Yamanaka et al. (2012) in which a 4-week period was investigated. Exercise at $20 \% 1 \mathrm{RM}$ with vascular occlusion resulted in an increase of $7.0 \%$ and $8.0 \%$ in squat and bench press performance, respectively in comparison to $3.2 \%$ and $4.9 \%$ in the control condition. These studies provide evidence that when high resistance exercise cannot be completed, or the load is high within a training schedule, but training needs to be sustained, $20 \% 1 \mathrm{RM}$ can be used to either maintain or improve muscular strength and hypertrophy when in combination with a traditional resistance exercise program.

Further studies included in the literature search have shown a direct improvement in performance using vascular occlusion, in the following study $70 \% 1 \mathrm{RM}$ with occlusion was implemented in comparison to $20-30 \% 1 \mathrm{RM}$ in previous studies, which may provide reasoning for the improvement in performance identified. Cook et al. (2014) investigated the use of vascular occlusion during 3 weeks of training. Vascular occlusion resulted in significantly greater improvements in bench press ( $\mathrm{p}=0.0044 ; 1.4 \pm 0.8 \%$ ), squat $\left(\mathrm{p}=1.03 \times 10^{-16} ; 2.0 \pm 0.6 \%\right)$, maximal 
sprint times $(\mathrm{p}=0.0162 ; 0.4 \pm 0.3 \%)$ and $\mathrm{CMJ}(\mathrm{p}=0.0003 ; 1.8 \pm 0.7 \%)$. In addition to previous research in this area, $\mathrm{T}$ and $\mathrm{C}$ concentrations were reported in response to training with vascular occlusion. Large to very large increases in $\mathrm{T}$ were observed in response to vascular occlusion training sessions (Effect Size [ES]: 1.50 to 2.19). An elevation in $\mathrm{T}$ has been shown to benefit performance, increasing adaptation from training, but also as a pre-competition strategy (Kilduff et al. 2013), therefore this may identify a further practical use for this strategy.

Although traditionally used as a training modality, responses to a single bout of low intensity resistance exercise with vascular occlusion have been reported (Table 2.2). When exploring records in more detail, the results are variable with studies showing lower EMG amplitude and repetitions complete (Karabulut et al. 2010; Loenneke et al. 2012), identifying no or an unknown effect (Takada et al. 2012) and records also showing a positive effect (Cayot et al. 2014). Fatela et al. (2016) potentially provide evidence for disparity in previous results. It was identified muscular activation, as well as neuromuscular fatigue varied as a function of relative occlusion intensity; $20 \% 1 \mathrm{RM}$ at 40,60 and $80 \%$ AOP. Torque only decreased in $80 \%$ AOP condition, except for the vastus medialis in the $40 \%$ condition, median frequency decreased in both muscles post exercise in all conditions. Median frequency decrements were of greater magnitude post exercise at higher levels of occlusion. Post reports, examining the interaction between occlusion and neuromuscular response, have shown this form of exercise implicates greater muscular activation to maintain the same total work output (Moritani et al. 1992; Shinohara et al. 1998; Takarada et al. 2000; Takarada et al. 2002; Yasuda et al. 2008). However, several methodological inconsistencies have generated conflicting findings, for instance, the large majority of research applied an arbitrary restrictive pressure for all participants.

In contrast, Neto et al. (2014) identified compromised muscle strength immediately after exercise with occlusion but when performed at $80 \%$ 1RM. However, no biochemical markers were measured and also the level of vascular occlusion was not controlled. Further studies have started to investigate the effect of higher intensity exercise with occlusion immediately prior to performance. Teixeira et al. (2017) applied vascular occlusion during the rest period between sets, whilst exercising and compared this to knee-extension exercise performed at 70\% 1RM without any vascular occlusion. The results infer vascular occlusion applied during the rest intervals increased lactate to a greater extent, possibly due to reduced restoration of ATP, however, EMG of the vastus laterallis indicate a decreased activation in all conditions. It was suggested that although previous studies have identified an increase in muscle activation when combined with low-load resistance exercise, when the load is already relatively high (i.e. $70 \% 1 \mathrm{RM}$ ) vascular occlusion does not further increase activation and may induce acute fatigue of both the contractile apparatus and CNS (Teixeira et al. 2017). These results have been supported by Dankel et al. 
(2017) again identifying that the stimulus of high-load training is sufficient to meet or exceed the stimulus which is provided by vascular occlusion when combined with low-load resistance exercise. A study by Takarada et al. (2000) assessed EMG during high and low-load resistance exercise, demonstrating vascular occlusion was able to augment EMG during elbow flexion exercise at $40 \%$ but not at $80 \% 1 \mathrm{RM}$, however, this study only assessed EMG during one set and the effectiveness of vascular occlusion has been reported to be reliant on the ability to pool metabolites within the muscle (Dankel et al. 2017)

Research suggests benefits of muscle activation when using vascular occlusion with low-load resistance exercise, however there is disparity when combined with high-low resistance exercise. To note, previous studies implementing vascular occlusion are inconsistent in the protocol applied, with intermittent occlusion completed during exercise and released during rest (e.g. Teixeira et al. 2017) compared to occlusion for the duration of exercise and rest (e.g. Neto et al. 2014). Alternative to the benefits of muscle activation on performance, previous research has identified a beneficial effect on hormone levels in response to vascular occlusion combined with exercise. Similar to high intensity protocols, low intensity occlusion has shown to increase serum hormone levels and blood lactate in men (Tanimoto et al. 2005; Takarada et al. 2000) and women (Kim et al. 2014) identifying growth hormone and $C$ increased in response to resistance exercise with and without vascular occlusion suggesting a similar increase in anabolic and catabolic hormone response. Research completed by Cook et al. (2014) implemented high-load (70\% 1RM) with vascular occlusion for a training program in males, but of interest, salivary $\mathrm{T}$ response was monitored immediately post sessions. An occlusion-dependent elevation in salivary $\mathrm{T}$ immediately after exercise was reported, potentially the result of an increased metabolic stress. This offers application as a pre-competition strategy to influence the circadian decline of $\mathrm{T}$ as discussed in section 2.2.2 and 2.2.3 to impact afternoon competition if performed in the morning prior to performance. 
Table 2.2: Characteristics of the studies examining the acute response of resistance exercise combined with vascular occlusion 2010-2017

\begin{tabular}{|c|c|c|c|c|c|c|}
\hline Author & Participants & Exercises & $\begin{array}{l}\text { Limbs occluded } \\
\text { \& Cuff pressure } \\
(\mathrm{mmHg})\end{array}$ & Intensity & $\begin{array}{l}\text { Protocol (sets, } \\
\text { reps, rest) }\end{array}$ & Performance benefit \\
\hline (Cayot et al. 2014) & $\begin{array}{l}7 \text { healthy, active } \\
\text { males }\end{array}$ & MIVC & $\begin{array}{l}\text { Legs }-130 \% \\
\text { resting SBP }\end{array}$ & $\begin{array}{l}20,40,60 \& \\
80 \% \mathrm{MVC}\end{array}$ & $\begin{array}{l}5 \mathrm{~s} \text { contraction, } 30 \mathrm{~s} \\
\text { recovery }\end{array}$ & $\begin{array}{l}\text { Applying VO 5min prior to } \\
\text { exercise enhances metabolic } \\
\text { stress, no difference in NM } \\
\text { activation }\end{array}$ \\
\hline $\begin{array}{l}\text { (Dankel et al. } \\
\text { 2017) }\end{array}$ & $\begin{array}{l}10 \text { male \& } \\
\text { female, } \\
\text { resistance trained }\end{array}$ & $\begin{array}{l}\text { Elbow } \\
\text { flexion }\end{array}$ & $\begin{array}{l}\text { Arms }-70 \% \\
\text { AOP }\end{array}$ & $70 \% 1 \mathrm{RM}$ & $\begin{array}{l}2 \text { sets to failure, } \\
3 \mathrm{~min} \text { rest between }\end{array}$ & $\begin{array}{l}\text { With VO less reps completed, } \\
\text { no difference in muscle } \\
\text { activation }\end{array}$ \\
\hline (Fatela et al. 2016) & $\begin{array}{l}14 \text { males, healthy } \\
\& \text { active }\end{array}$ & $\begin{array}{l}\text { Knee } \\
\text { extension }\end{array}$ & $\begin{array}{l}\text { Legs }-40,60 \\
80 \% \text { AOP }\end{array}$ & $20 \% 1 \mathrm{RM}$ & $\begin{array}{l}30-15-15-15,30 \mathrm{~s} \\
\text { rest between bouts }\end{array}$ & $\begin{array}{l}\text { Muscle activation and NM } \\
\text { fatigue vary relative to } \\
\text { restrictive level }\end{array}$ \\
\hline $\begin{array}{l}\text { (Husmann et al. } \\
\text { 2017) }\end{array}$ & $\begin{array}{l}17 \text { resistance } \\
\text { trained males }\end{array}$ & $\begin{array}{l}\text { Knee } \\
\text { extension }\end{array}$ & Legs - $60 \%$ AOP & $30 \% 1 \mathrm{RM}$ & $\begin{array}{l}30-15-15-15,30 \mathrm{~s} \\
\text { rest between bouts }\end{array}$ & $\begin{array}{l}\text { Decrease MVT, increase } \\
\text { muscle fatigue immediately, } \\
\text { but diminished after } 2 \mathrm{~min}\end{array}$ \\
\hline $\begin{array}{l}\text { (Karabulut et al. } \\
\text { 2011) }\end{array}$ & 14 healthy men & $\begin{array}{l}\text { Leg } \\
\text { extensions }\end{array}$ & Legs-SBPx1.44 & $20 \% 1 \mathrm{RM}$ & $\begin{array}{l}5 \times 20 \text { reps, } 30 \mathrm{~s} \text { rest } \\
\text { between bouts }\end{array}$ & $\begin{array}{l}\text { Greater decrease in MVC } \\
\text { following exercise with VO }\end{array}$ \\
\hline $\begin{array}{l}\text { (Lauver et al. } \\
\text { 2017) }\end{array}$ & $\begin{array}{l}24 \text { healthy } \\
\text { participants }\end{array}$ & $\begin{array}{l}\text { Knee } \\
\text { extension }\end{array}$ & $\begin{array}{l}\text { Legs }-130 \% \\
\text { SBP }\end{array}$ & MVC & $\begin{array}{l}6 \times 10 \text { reps, } 2 \text { min } \\
\text { rest between }\end{array}$ & $\begin{array}{l}\text { NM and metabolic responses } \\
\text { different with VO }\end{array}$ \\
\hline $\begin{array}{l}\text { (Loenneke et al. } \\
\text { 2012) }\end{array}$ & $\begin{array}{l}20 \text { healthy, } \\
\text { active male \& } \\
\text { females }\end{array}$ & $\begin{array}{l}\text { Leg } \\
\text { extension }\end{array}$ & $\begin{array}{l}\text { Legs }- \text { not } \\
\text { reported }\end{array}$ & $30 \% 1 \mathrm{RM}$ & $2 \mathrm{x}$ sets to failure & $\begin{array}{l}\text { Failure occurred sooner with } \\
\text { VO with similar metabolic } \\
\text { stress }\end{array}$ \\
\hline $\begin{array}{l}\text { (Loenneke et al. } \\
\text { 2013) }\end{array}$ & $\begin{array}{l}16 \text { healthy active } \\
\text { males \& females }\end{array}$ & $\begin{array}{l}\text { Knee } \\
\text { extension }\end{array}$ & Leg $-60 \%$ AOP & $30 \% 1 \mathrm{RM}$ & $\begin{array}{l}30-15-15 \text { reps, } 30 \mathrm{~s} \\
\text { rest }\end{array}$ & $\begin{array}{l}\mathrm{VO}+\text { exercise does not result } \\
\text { in prolonged decrements in } \\
\text { torque }\end{array}$ \\
\hline (Neto et al. 2014) & $\begin{array}{l}12 \text { Jiu-Jitsu } \\
\text { fighters }\end{array}$ & Squat & Legs - 110 & $80 \% 1 \mathrm{RM}$ & To failure & $\begin{array}{l}\mathrm{HI}+\mathrm{VO} \text { compromise muscle } \\
\text { strength after exercise }\end{array}$ \\
\hline $\begin{array}{l}\text { (Takada et al. } \\
\text { 2012) }\end{array}$ & $\begin{array}{l}12 \text { male trained } \\
\text { participants }\end{array}$ & $\begin{array}{l}\text { Plantar } \\
\text { flexion }\end{array}$ & $\begin{array}{l}\text { Legs - not } \\
\text { reported }\end{array}$ & $\begin{array}{l}20 \% 1 \mathrm{RM} \\
\text { or } 65 \% 1 \mathrm{RM}\end{array}$ & $\begin{array}{l}\text { 30reps per min for } \\
2 \mathrm{~min}\end{array}$ & $\begin{array}{l}\text { No difference in fast twitch } \\
\text { recruitment }\end{array}$ \\
\hline
\end{tabular}




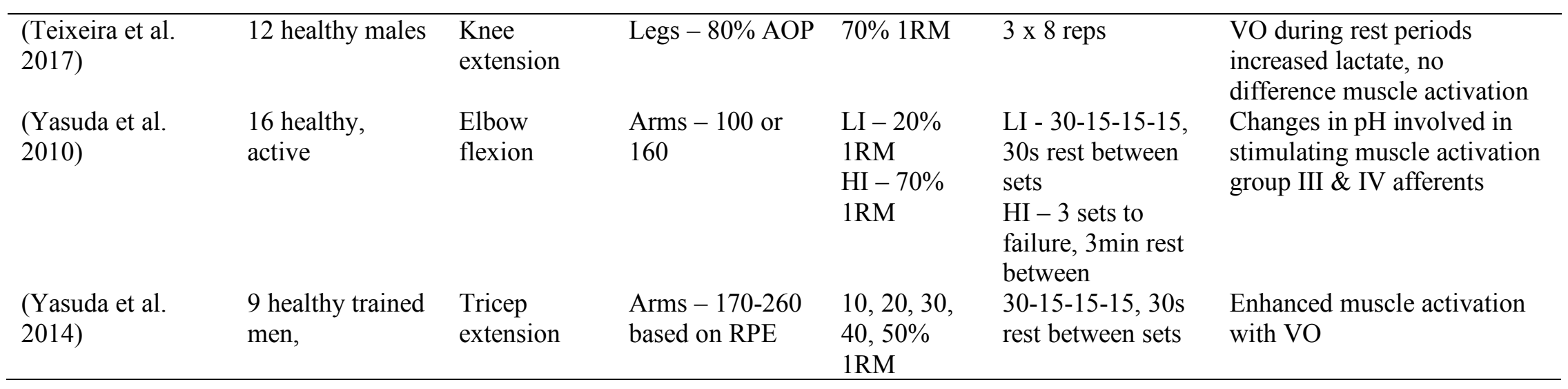

MIVC - maximal isometric voluntary contraction; MVC; maximal voluntary contraction; AOP - arterial occlusion pressure; HI - high intensity, LI - low intensity, VO - vascular occlusion, 1RM - 1 repetition maximum; GH - growth hormone; NM - neuromuscular; MVT - maximal voluntary torque 


\subsubsection{Identified mechanisms for the use of vascular occlusion and resistance exercise}

Consequence of the additive effects of mechanical and metabolic stress (Pearson \& Hussain 2015), it has been hypothesized that resistance exercise with vascular occlusion signals a number of mechanisms for induction of muscle activation and growth. Reviews by Kacin et al. (2015), Pope et al. (2013) and Heitkamp (2015) summarised the potential physiological responses of resistance exercise with vascular occlusion: (i) hypoxia-induced additional or preferential recruitment of fasttwitch muscle fibers, (ii) greater duration of metabolic acidosis via the trapping and accumulation of intramuscular protons $(\mathrm{H}+$ ions) and stimulation of metaboreceptors, possibly eliciting an exaggerated acute systemic hormonal response, (iii) external pressure-induced differences in contractile mechanics and sarcolemmal deformation, resulting in enhanced growth factor expression and intracellular signaling, (iv) metabolic adaptations to the fast glycolytic system that stem from compromised $\mathrm{O}_{2}$ delivery, (vi) production of ROS that promotes tissue growth, (vii) gradient-induced reactive hyperemia after removal of the external pressure, which induces intracellular swelling and stretches cytoskeletal structures that may promote tissue growth, and (vii) activation of myogenic stem cells with subsequent myonuclear fusion with mature muscle fibers (Kacin et al. 2015) .

Expanding on the summarised mechanisms, a primary mechanism identified is the preferential recruitment of fast twitch fibres due to the effect of the hypoxic-induced environment. Heavy load typically used in resistance exercise programs are thought to be a requirement to ensure that most motor units are recruited and thus exposed to the stimulus of strength training (Wernbom et al. 2009). During resistance exercise, motor units and hence muscle fibres are recruited according to the size principle (Dideriksen \& Farina 2013), to increase muscle mass and strength, it is important to activate type II muscle fibres during training (Häkkinen \& Keskinen 1989). Changes in blood flow to skeletal muscle can alter the level of supply of substrates, the removal of metabolites, and fatigue, which significantly affects the recruitment and activation of muscle fibres needed to perform under specific conditions (Karabulut \& Perez 2013). Reducing blood flow to the active skeletal muscle during exercise may affect the pattern and the number of skeletal muscle fibres recruited and therefore the level of neuromuscular activation and adaptation (Karabulut \& Perez 2013).

Research has demonstrated that recruitment of fast-twitch muscle fibres is also possible at low intensities with vascular occlusion, likely due to inadequate $\mathrm{O}_{2}$ supply and high metabolite accumulation (Moritani et al. 1992; Meyer 2006). Both reduced $\mathrm{O}_{2}$ and metabolite accumulation can increase type II fibre recruitment through the stimulation of group III and IV afferents, causing inhibition of alpha motorneuron, increasing fibre recruitment to maintain muscular force and protect against conduction failure (Yasuda et al. 2010; Loenneke et al. 2011). It is the progressive 
increase in the recruitment of type II motor units that elevates the stress on these units and consequently produces adaptation in the form of hypertrophy (Kawada 2005). As a result, overall exercise volume may play a significant role in the muscular adaptations (Fahs et al. 2012). Theoretically, vascular occlusion exercise to volitional fatigue would produce a greater metabolic accumulation and lead to greater adaptations than exercise terminated prior to fatigue.

Similarly, Kacin \& Strazar (2011) evaluated vastus medialis and rectus femoris EMG activity during dynamic knee extensions performed to repetition failure under 2 different conditions i) control $15 \%$ of maximal voluntary contraction without vascular occlusion, ii) $15 \%$ of maximal voluntary contraction with vascular occlusion at $230 \mathrm{mmHg}$. The authors found similar increases in EMG amplitude during both testing conditions. These results might indicate a greater sensory feedback or perception of exertion during vascular occlusion that inhibits motor drive and subsequent levels of muscle activity (Loenneke et al. 2011). Whereas Fujita et al. (2007) found that resistance exercise with vascular occlusion compared to a work matched protocol without vascular occlusion demonstrated increased S6K1 phosphorylation following occlusion, which primarily occurs in type II muscle fibres (Koopman et al. 2006), indirectly linking vascular occlusion to increased recruitment of fast twitch fibres. Hypoxia induced muscular activation is supported by a study conducted by Katayama et al. (2010), where it was determined that hypoxic conditions were responsible for changes in muscle fibre activation from type I to type II as well as increase motor unit recruitment. Since slow twitch muscle fibres rely heavily on oxidative phosphorylation, they may have been unable to contribute to isometric force production due to reduced blood flow to skeletal muscles during the post exercise maximal voluntary contraction trials with vascular occlusion.

However, some investigations have failed to demonstrate increased muscle activation during resistance exercise with vascular occlusion. Research suggests that low intensity vascular occlusion with resistance exercise does not facilitate muscle activation of the same magnitude as higher intensity resistance exercise without vascular occlusion (Suga et al. 2009; Cook et al. 2013). Reports suggest it is unlikely that low intensity vascular occlusion with resistance exercise will stimulate all of the high-threshold motor units as experienced during high intensity resistance exercise, resulting in lower chronic neuromuscular responses following low intensity exercise with vascular occlusion (Scott et al. 2014). This should be considered when implementing this strategy to athletes; it may not benefit sports where rapid force production is required (Scott et al. 2015).

Alternatively, vascular occlusion has been reported to increase intramuscular pressure, thus affecting blood circulation and resulting in even higher lactate build up. The increased metabolic stress caused by accumulation of metabolites is thought to be the result of reduced venous outflow. 
By combining exercise with vascular occlusion, it has been identified that the ischemic (Takano et al. 2005) intramuscular environment induces a greater rate of ATP hydrolysis, exaggerated PCr depletion (Suga et al. 2009), decreased pH (Suga et al. 2009; Suga et al. 2010) and an increased lactate response (Takano et al. 2005; Fujita et al. 2007; Reeves et al. 2006) to cause greater response and adaptation. Metabolic stress has been defined as the decrease in ATP, depletion of $\mathrm{PCr}$, increased inorganic $\mathrm{Pi}$, increased ADP/ATP ratio, increased adenosine monophosphate (AMP) production, and decreased intramuscular $\mathrm{pH}$ and accumulation of lactate. The importance of metabolic stress for hypertrophy has been highlighted by Goto et al. (2003) comparing the effects of 2 volume/intensity matched resistance exercise protocols, the only difference being one protocol included $30 \mathrm{~s}$ rest between sets. Blood lactate concentrations were significantly higher following the without-rest protocol and following 12 weeks of training muscle cross sectional area increased significantly compared to no difference in the rest condition, thus indicating a direct link between metabolic stress and muscle hypertrophy. Furthermore, high intramuscular proton concentrations can inhibit $\mathrm{Ca}^{2+}$ release from the sarcoplasmic reticulum, disrupting excitationcontraction coupling of myofilaments, thereby reducing tension development as manifested in peripheral muscular fatigue (Favero et al. 1993).

Adaptive responses to resistance training with hypoxia (Scott et al. 2014), propose that a localised hypoxic stimulus may play an important role; downstream of the hypoxic stimulus, it is likely that a greater accumulation of metabolites, due to both increased production in the more hypoxic state and limited removal due to vascular occlusion, acts as a primary moderator of the anabolic response to this form of exercise (Schoenfeld 2013). This accumulation may cause increased muscle fibre recruitment (Takarada et al. 2000; Yasuda et al. 2009), along with muscle cell swelling and altered anabolic/catabolic signalling (Fry et al. 2010; Fujita et al. 2007; Laurentino et al. 2012) and elevated endocrine responses (Takarada et al. 2000).

However, a recent review by Loenneke et al. (2011) indicated a minimal $\mathrm{T}$ response to vascular occlusion with resistance exercise as supported by previous research (e.g. Abe et al. 2006; Fujita et al. 2007; Reeves et al. 2006). These results may be attributable to the exercises selected; larger muscle groups have been associated with an increase in T. Furthermore, the low volume or intensity of the exercise protocols used may provide explanation for the difference in results. This has been demonstrated with research completed by Cook et al. (2014), in which large to very large increases in $\mathrm{T}$ were observed in response to vascular occlusion training sessions due to exercise being complete at $70 \% 1 \mathrm{RM}$ rather than low intensity (20-30\% 1RM) as used in previous studies. Further explanation for this disparity in results is apparent when comparing vascular occlusion protocols utilised. In the studies identifying no elevation in $\mathrm{T}$, continuous occlusion rather than 
intermittent occlusion and also the studies utilised blood rather than salivary sampling, which could influence steroid hormone concentrations (Credeur et al. 2010).

\subsubsection{Limb IPC and athletic performance}

Within the literature, 17 out of 24 studies identified improvement in exercise performance, including; $5 \mathrm{~km} \mathrm{TT}$, total exercise time and $100 \mathrm{~m}$ swim TT (Table 2.4).

Due to the direct impact of time to exhaustion on exercise performance, research has recently investigated the use of limb IPC to extend time to exhaustion. Eight studies (Barbosa et al. 2015; Clevidence et al. 2012; Crisafulli et al. 2011; De Groot et al. 2010; Hittinger et al. 2015; Kido et al. 2015; Sabino-Carvalho et al. 2016; Tanaka et al. 2016) have investigated the use of limb IPC on time to exhaustion (Table 2.3). Six of the studies identified improvement in performance, for instance De Groot et al. (2010) examined and identified a beneficial effect of limb IPC on $\mathrm{O}_{2}$ consumption (3\% improvement) and workload (maximal power output $372 \pm 59 \mathrm{~W}$ ) during a maximal cycling test to exhaustion (Table 2.3). However, in contrast, research complete by Clevidence et al. (2012) implemented the same limb IPC protocol (Table 2.3) but documented no improvement in performance. Differences in the exercise test to exhaustion provide somewhat of an explanation for the contrast in results. The reported workload during the initial submaximal component of the incremental test varied significantly with De Groot et al. (2010) initiating exercise at $50 \mathrm{~W}$ for four min compared to $121 \pm 13.5 \mathrm{~W}$ for five min with the elevation remaining higher throughout the test complete by Clevidence et al. (2012). Thus, a higher workload was completed during the initial stages of the test to exhaustion and may explain the inconsistencies in results.

Tanaka et al. (2016) examined the effects of limb IPC on local muscle endurance during fatiguing isometric exercise. Endurance time to task failure was significantly longer following limb IPC than in the control, although quadriceps EMG activity was not significantly different between conditions, deoxygenation dynamics in the quadriceps vastus lateralis muscle was significantly faster in limb IPC than in the control. This suggests enhanced muscular endurance during fatiguing isometric exercise as a result of accelerated muscle deoxygenation dynamics and enhanced mitochondrial metabolism. This is supported by Paradis-Deschenes et al. (2016) identifying three cycles of limb IPC immediately increased muscle perfusion and $\mathrm{O}_{2}$ uptake, conducive to higher repeated force capacity in strength-trained athletes.

Physiologically Kido et al. (2015) and Hittinger et al. (2015) assessed the systemic and local $\mathrm{O}_{2}$ dynamics in response to limb IPC. Disparity in results may be explained between the variation in 
study design and participants examined. In addition to variation in performance test, the cuff pressure applied and total duration of ischemia were not comparable (e.g. Cuff pressure: $>300 \mathrm{mmHg}$ versus $10-20 \mathrm{mmHg}>$ systolic blood pressure, respectively). Furthermore, Kido et al. (2015) reported an improvement in performance in 15 healthy male participants, whereas Hittinger et al. (2015) did not find limb IPC improved subsequent performance, however this was with 15 highly trained male cyclists and triathletes suggesting the training history of the participant may impact the efficacy of limb IPC on athletic performance. However, none of the performance tests from the previously discussed studies were specific to the peak and mean power and duration required for athletic performance in competition, therefore further research in this area may provide increased insight and practical application to the use of limb IPC within sport.

Exercise such as repeated six s or $30 \mathrm{~s}$ sprints is frequently performed in sports performance. This is associated with an increase venous lactate, reduction of arterial $\mathrm{pH}$ and a cause of fatigue (Hargreaves et al. 1998). Limb IPC may improve this type of maximal performance as a result of the opening of $\mathrm{K}_{\mathrm{ATP}}$ channels in the mitochondria by the release of adenosine (Lalonde \& Curnier 2014) consequently improving the metabolic pathway. However, there has been disparity within the results of limb IPC and repeated sprint performance, including a detrimental (Gibson et al. 2013; Paixão et al. 2014), no effect (Gibson et al. 2013; Lalonde \& Curnier 2014) or positive effect (Patterson et al. 2015)

For instance, Gibson et al. (2013) identified no beneficial effect of limb IPC on repeated sprints $(3 \times 30 \mathrm{~m})$ and a potential small negative effect in female participants. It is postulated the protocol implemented (Table 2.3) was insufficient to have an accumulation of metabolites to initiate a biochemical cascade associated with limb IPC (Downey et al. 2007). Furthermore, Gibson et al. (2013) suggested the detriment in female participants could be due to methodological issues of thigh circumference and associated level of limb IPC or participants' perception of discomfort and individual pain tolerance. In addition, Paixão et al. (2014) identified a reduced maximal power in the first wingate test following limb IPC. However, the limb IPC protocol varied with a pressure cuff of $250 \mathrm{mmHg}$ being utilised with no individualisation and may be attributable to the decreased performance. Furthermore, both studies only allowed 12-15 min rest between limb IPC and the onset of the performance test. Records that identified a benefit of performance concluded the limb IPC protocol 30 to $90 \mathrm{~min}$ before the onset of performance, a shorter rest between limb IPC and performance may have a detrimental effect on performance resulting from lowered PCr, ATP and buffer capacity immediately after limb IPC and therefore the outcome on athletic performance may be different following an extended rest period. 
This may be confirmed by research completed by Patterson et al. (2015), although not in trained participants, an improved peak and mean power output (2-4 \%) during the early stages (sprint 13 ) of repeated sprint cycling (12x6 s) was reported following the use of limb IPC, completed 45 min before the start of the exercise test. The variation in methodologies regarding time, distance and recovery of repeated sprint exercise protocols (Table 2.3) in addition to differences in the limb IPC protocol do not allow conclusions or recommendations to be drawn on the effect of limb IPC on repeated sprint exercise performance. Therefore, further research is required on the use of limb IPC before repeated sprints in highly trained athletes ensuring a prolonged rest period is included between limb IPC and the onset of performance.

Within sports competition, it is common to perform single, maximal efforts such as in athletics and swimming. Contradicting results have been identified within this area of performance; Bailey et al. (2012) reported improved $5 \mathrm{~km}$ running TT performance, whereas Tocco et al. (2015) failed to identify an improvement in $5 \mathrm{~km}$ running TT performance after limb IPC (Table 2.3). There were differences in cuff pressure applied, the number of cycles of ischemia and also the time between conclusion of limb IPC and performance test was notably different; five min versus 45 min respectively. This potentially explains the disparity in findings as reported lower levels of PCr, ATP and reduced buffer capacity after limb IPC may impair performance reliant of anaerobic metabolism (Salvador et al. 2016) and highlights the importance of appropriate timing for the implementation of limb IPC in relation to the time of athletic performance, consistent with research investigating repeated sprint performance.

To date, three studies (Jean-St-Michel et al. 2011; Lisbôa et al. 2017; Marocolo et al. 2015) (Table 2.3) have identified a positive effect of implementing limb IPC at a single time point prior to swimming performance. For example, Jean-St-Michel et al. (2011) reported that five min of ischemia followed by five min of reperfusion, repeated for four cycles, implemented $45 \mathrm{~min}$ prior to $100 \mathrm{~m}$ swimming TT improved personal best swimming times by $1.1 \%$. However, no physiological measures are available to determine the underlying mechanism responsible for the improvement in performance. In addition to the maximal competition effort, a submaximal performance test was completed; however, no beneficial effect of limb IPC was identified. This is potentially explained by the differences in pathways of energy utilisation during submaximal and maximal exertion (Jean-St-Michel et al. 2011). During submaximal exercise, mainly the aerobic oxidative pathways produce energy, whereas during maximal performance energy is produced not only by the breakdown of PCr but also by the anaerobic glycolytic pathway (Wells et al. 2009) in addition to the aerobic oxidative system. Furthermore maximal exercise represents a physiologic form of ischemic injury and therefore maybe modified by limb IPC implemented 
pre-competition (Jean-St-Michel et al. 2011). Therefore, this suggests the benefit of limb IPC is influenced by the intensity of exercise performed.

Most recently, Lisbôa et al. (2017) applied limb IPC one $h$, two $h$ and eight $h$ preceding a $50 \mathrm{~m}$ TT performance, with performance improvements of $1.0 \%$ and $1.2 \%$ in two $\mathrm{h}$ and eight $\mathrm{h}$ conditions, respectively. The previous research relating to limb IPC and swimming performance has investigated the effects of the early phase of limb IPC on performance as application has been $<12 \mathrm{~h}$ prior to performance. However, for short duration events (i.e. 10-90 s), a recent metaanalysis showed that a longer duration between limb IPC and exercise resulted in a higher effect size; suggesting that limb IPC may be dependent on the timing of the preconditioning strategy relative to the start of subsequent performance (Salvador et al. 2016). However, only one study (Seeger et al. 2017) relating to sports performance has differentiated between the observed early and late phase of limb IPC reported within the clinical literature (see section 2.3.1), exposing 12 healthy participants to limb IPC either immediately or $24 \mathrm{~h}$ prior to a self-paced $5 \mathrm{~km}$ running TT; no differences in finish time after either limb IPC were found in comparison to the control, but suggested that at an individual level, the effects of limb IPC $24 \mathrm{~h}$ prior to TT were closely related to the effects of limb IPC. However, the study further stated that participants started at a higher pace during the $24 \mathrm{~h}$ limb IPC, although ending with a slower pace; this may suggest application for anaerobic exercise to improve performance.

\subsubsection{Identified mechanisms for the use of IPC as a pre-competition strategy}

There are many physiological determinants that can affect performance, especially across different sporting activities. The use of limb IPC has been explored across varying durations and intensities of exercise as previously outlined, thus highlighting the potential of different mechanisms that could be improving performance.

Within different sporting endeavours there are three distinct yet closely integrated processes that operate together to meet the energy requirements of muscle (Gastin 2001). The splitting of the stored phosphagens, ATP and PCr and the nonaerobic breakdown of carbohydrates comprise the anaerobic energy system (Gastin 2001). This pathway is capable of regenerating ATP at high rates, resulting in large muscle power output (Gastin 2001) for explosive, single effort activities as explored in the above studies describing anaerobic race specific and muscular activation. The capacity of the anaerobic system is limited by the amount of energy that can be released in a single exercise bout (Gastin 2001). In contrast, the aerobic system is capable of producing large amounts of ATP, yet is rate limited by oxidative phosphorylation and the respiratory and cardiovascular systems to deliver $\mathrm{O}_{2}$ to the muscle (Gastin 2001). These systems are able to cope with high, often sustained and diverse energy demands placed on them during sporting activities (Gastin 2001), 
including time to exhaustion. However, there are several factors that can affect fatigue and therefore performance in sport.

Exercise performance in highly trained athletes has been reported to be limited by tissue hypoxemia and acidosis due to a decrease in arterial $\mathrm{pH}$ and substantial rise in venous lactate (Wells et al. 2009), which may therefore represent a type of ischemia-reperfusion stress modifiable by limb IPC (Jean-St-Michel et al. 2011). Exercise-induced arterial hypoxemia may be a significant contributor to the development of fatigue in skeletal, respiratory and cardiac muscles (Noakes 2000) responsible for the physiological limitation in maximal exercise (Jean-St-Michel et al. 2011). Literature has presented limb IPC as a potential strategy to be incorporated before exercise to generate resistance in tissues to the adverse metabolic effects of high intensity exercise (Jean-St-Michel et al. 2011). In the instance where studies were not associated with positive outcomes, it is possible that this was due to variation in the protocol utilised regarding cuff pressure, width and duration; as reported in the previous section all of these variables can have a large impact on the effectiveness and outcome of limb IPC.

Several mechanisms have been proposed to explain the effect of limb IPC on athletic performance. Research has reported the positive effects of limb IPC appear to depend on specific metabolites' (adenosine, bradykinin or opioids) reaching a critical level (Downey et al. 2007). It has been speculated, consistent with clinical literature, that adenosine released during occlusion provides a cardiac protection and stimulates adenosine A1-receptors, which have a protective effect even after adenosine has been removed (Liu et al. 1991). Furthermore, adenosine is a vasodilator that contributes to the vasodilation response during exercise (Joyner \& Proctor 1999); therefore adenosine may help to optimise blood supply during exercise (De Groot et al. 2010), increasing $\mathrm{O}_{2}$ delivery, removal of metabolic waste (Vaile et al. 2011) and repletion of ATP (Connolly et al. 2003). Research suggests if limb IPC and/or the exercise challenge is not severe enough to cause an accumulation of metabolites (adenosine, bradykinin, opioids) required to reach a critical threshold, this may explain the absence of the beneficial effects of limb IPC on athletic performance (Liu et al. 1991). 
Table 2.3 Characteristics of the studies identifying the effect of limb ischemic preconditioning on exercise performance

\begin{tabular}{|c|c|c|c|c|c|c|c|c|}
\hline Study (date) & Participants & $\begin{array}{l}\text { Sport } \\
\text { specific }\end{array}$ & $\begin{array}{l}\text { Series of } \\
\text { occlusion }\end{array}$ & Pressure & $\begin{array}{l}\text { Area } \\
\text { occluded }\end{array}$ & $\begin{array}{l}\text { Time to } \\
\text { test }\end{array}$ & Performance test & Findings \\
\hline $\begin{array}{l}\text { (Bailey et al. } \\
\text { 2012) }\end{array}$ & 13 healthy & Running & $4 \times 5: 5 \mathrm{~min}$ & $220 \mathrm{mmHg}$ & Thigh & $90 \mathrm{~min}$ & $\begin{array}{l}\text { Incremental } 5 \mathrm{~km} \\
\text { running TT }\end{array}$ & $\begin{array}{l}34 \mathrm{~s}(2.3 \% \text { reduction }) \\
\text { improvement in } 5 \mathrm{~km}\end{array}$ \\
\hline $\begin{array}{l}\text { (Barbosa et } \\
\text { al. 2015) }\end{array}$ & 13 healthy & Handgrip & $3 \times 5: 5 \mathrm{~min}$ & $200 \mathrm{mmHg}$ & Thigh & & $\begin{array}{l}\text { Constant load } \\
\text { rhythmic hand grip } \\
\text { \& MVC }\end{array}$ & $\begin{array}{l}\text { Increase time to task } \\
\text { failure by } 11.2 \%\end{array}$ \\
\hline $\begin{array}{l}\text { (Clevidence } \\
\text { et al. 2012) }\end{array}$ & 12 cyclists & Cycling & $3 \times 5: 5 \mathrm{~min}$ & $220 \mathrm{mmHg}$ & Thigh & $5 \mathrm{~min}$ & $\begin{array}{l}\text { Incremental to } 90 \% \\
\text { max PO until } \\
\text { exhaustion }\end{array}$ & $\begin{array}{l}\text { No improvement in } \\
\text { submax or time to } \\
\text { exhaustion }\end{array}$ \\
\hline $\begin{array}{l}\text { (Cocking et } \\
\text { al. 2017) }\end{array}$ & 12 cyclists & Cycling & $\begin{array}{l}4 \times 5: 5 \mathrm{~min} \\
8 \times 5: 5 \mathrm{~min}\end{array}$ & $220 \mathrm{mmHg}$ & $\begin{array}{l}\text { Thigh \& } \\
\text { arms }\end{array}$ & $30 \mathrm{~min}$ & $375 \mathrm{KJ}$ cycling TT & $\begin{array}{l}4 \times 5: 5 \text { min resulted in } \\
17 \mathrm{~s} \text { faster TT }\end{array}$ \\
\hline $\begin{array}{l}\text { (Crisafulli et } \\
\text { al. 2011) }\end{array}$ & 17 healthy & Cycling & $3 \times 5: 5 \mathrm{~min}$ & $\begin{array}{l}50 \mathrm{mmHg}> \\
\text { SBP }\end{array}$ & Thigh & $5 \mathrm{~min}$ & $\begin{array}{l}\text { Incremental to } \\
\text { exhaustion. Total } \\
\text { exercise time, } \\
\text { maximal PO }\end{array}$ & $\begin{array}{l}\text { Improvements total } \\
\text { exercise time, } 4 \% \\
\text { increase PO }\end{array}$ \\
\hline $\begin{array}{l}\text { (Cruz et al. } \\
2015 \text { ) }\end{array}$ & 15 cyclists & Cycling & $4 \times 5: 5 \mathrm{~min}$ & $220 \mathrm{mmHg}$ & Thigh & $33 \mathrm{~min}$ & 60 s sprint cycle & $\begin{array}{l}\text { Improved performance } \\
2.1 \%\end{array}$ \\
\hline $\begin{array}{l}\text { (De Groot et } \\
\text { al. 2010) }\end{array}$ & 15 cyclists & Cycling & $3 \times 5: 5 \mathrm{~min}$ & $220 \mathrm{mmHg}$ & Thigh & $5 \mathrm{~min}$ & $\begin{array}{l}\text { Incremental to } \\
\text { exhaustion }\end{array}$ & $\begin{array}{l}\text { Improved maximal } \\
\text { oxygen consumption } \\
3 \% \text { and PO by } 1.6 \% \text {. }\end{array}$ \\
\hline $\begin{array}{l}\text { (Gibson et } \\
\text { al. 2013) }\end{array}$ & $\begin{array}{l}25 \text { team } \\
\text { sport } \\
\text { athletes }\end{array}$ & Running & $3 \times 5: 5 \mathrm{~min}$ & $220 \mathrm{mmHg}$ & Thigh & $11.1 \mathrm{~min}$ & $\begin{array}{l}3 \mathrm{max} \text { sprints over } \\
30 \mathrm{~m}, 1 \mathrm{~min} \text { rest, }\end{array}$ & $\begin{array}{l}\text { No effect on sprint } \\
\text { speed, small negative } \\
\text { effect on female athletes }\end{array}$ \\
\hline
\end{tabular}




\begin{tabular}{|c|c|c|c|c|c|c|c|c|}
\hline $\begin{array}{l}\text { (Hittinger et } \\
\text { al. 2015) }\end{array}$ & $\begin{array}{l}15 \text { male } \\
\text { cyclists \& } \\
\text { triathletes }\end{array}$ & Cycling & $4 \times 5: 5 \mathrm{~min}$ & $\begin{array}{l}10-20 \mathrm{mmHg}> \\
\text { SBP }\end{array}$ & Thighs & $45 \mathrm{~min}$ & $\begin{array}{l}10 \text { min submax at } \\
55 \% \text { altitude } \\
\text { specific } \mathrm{W}_{\text {peak }} \& \text { test } \\
\text { to exhaustion }\end{array}$ & $\begin{array}{l}\text { No change in } \\
\text { performance }\end{array}$ \\
\hline $\begin{array}{l}\text { (Jean-St- } \\
\text { Michel et al. } \\
\text { 2011) }\end{array}$ & $\begin{array}{l}16 \\
\text { swimmers }\end{array}$ & Swimming & $4 \times 5: 5 \mathrm{~min}$ & $\begin{array}{l}15 \mathrm{mmHg}> \\
\mathrm{SBP}\end{array}$ & Arms & $45 \mathrm{~min}$ & $100 \mathrm{~m} \max \mathrm{TT}$ & $\begin{array}{l}\text { Improvement } 0.7 \mathrm{~s} \text {, no } \\
\text { improvement submax }\end{array}$ \\
\hline $\begin{array}{l}\text { (Kido et al. } \\
\text { 2015) }\end{array}$ & 15 healthy & Cycling & $3 \times 5: 5 \mathrm{~min}$ & $>300 \mathrm{mmHg}$ & Thigh & $12 \mathrm{~min}$ & $\begin{array}{l}\text { Incremental step test } \\
\text { to exhaustion }\end{array}$ & $\begin{array}{l}\text { Increase time to } \\
\text { exhaustion }\end{array}$ \\
\hline $\begin{array}{l}\text { (Kjeld et al. } \\
\text { 2014) }\end{array}$ & $\begin{array}{l}31 \text { divers \& } \\
\text { rowers }\end{array}$ & $\begin{array}{l}\text { Diving \& } \\
\text { rowing }\end{array}$ & $4 \times 5: 5 \mathrm{~min}$ & $\begin{array}{l}40 \mathrm{mmHg}> \\
\mathrm{SBP}\end{array}$ & Arm & $30 \mathrm{~min}$ & $\begin{array}{l}1 \mathrm{~km} \text { row and } \\
\text { distance swam } \\
\text { underwater }\end{array}$ & $\begin{array}{l}\text { Decreased } 1 \mathrm{~km} \text { rowing } \\
\text { time and increased } \\
\text { distance swam }\end{array}$ \\
\hline $\begin{array}{l}\text { (Kraus et al. } \\
\text { 2015) }\end{array}$ & $\begin{array}{l}\text { Total } 43 \\
\text { healthy }\end{array}$ & Cycling & $4 \times 5: 5 \mathrm{~min}$ & Not reported & Arm & $10 \mathrm{~min}$ & $4 \times 30$ s wingate test & $\begin{array}{l}\text { Bilateral but not } \\
\text { unilateral IPC improved } \\
\text { performance }\end{array}$ \\
\hline $\begin{array}{l}\text { (Lalonde \& } \\
\text { Curnier } \\
\text { 2014) }\end{array}$ & 15 healthy & Cycling & $4 \times 5: 5 \mathrm{~min}$ & $50 \mathrm{mmHg}>\mathrm{SBP}$ & Arm & $\begin{array}{l}10 \\
65 \mathrm{~min}\end{array}$ & $\begin{array}{l}6 \times 6 \text { sec sprint }- \\
2 \text { min active rest }\end{array}$ & No improvement \\
\hline $\begin{array}{l}\text { (Lisbôa et al. } \\
\text { 2017) }\end{array}$ & $\begin{array}{l}11 \text { male } \\
\text { swimmers }\end{array}$ & Swimming & $4 \times 5: 5 \mathrm{~min}$ & $\begin{array}{l}220 \text { thighs, } \\
180 \text { arms }\end{array}$ & $\begin{array}{l}\text { Thigh \& } \\
\text { arm }\end{array}$ & 1,2 or $8 h$ & 50m swim TT & $\begin{array}{l}2 \mathrm{~h}-1.0 \% \text { and } 8 \mathrm{~h}- \\
1.2 \% \text { improvement in } \\
\text { performance }\end{array}$ \\
\hline $\begin{array}{l}\text { (Marocolo et } \\
\text { al. 2015) }\end{array}$ & $\begin{array}{l}15 \\
\text { swimmers }\end{array}$ & Swimming & $4 \times 5: 5 \mathrm{~min}$ & $220 \mathrm{mmHg}$ & Arm & $\begin{array}{l}\text { Not } \\
\text { reported }\end{array}$ & 100m swim TT & $\begin{array}{l}\text { Improved performance } \\
\text { in IPC and SHAM } \\
\text { compared to CON }\end{array}$ \\
\hline $\begin{array}{l}\text { (Marocolo et } \\
\text { al. 2016) }\end{array}$ & 13 males & $\begin{array}{l}\text { Resistance } \\
\text { exercise }\end{array}$ & $4 \times 5: 5 \mathrm{~min}$ & $200 \mathrm{mmHg}$ & Thigh & $\begin{array}{l}\text { Not } \\
\text { reported }\end{array}$ & $\begin{array}{l}\text { 3RM sets leg } \\
\text { extension at } 12 \mathrm{RM} \\
\text { load }\end{array}$ & $\begin{array}{l}\text { Improved performance } \\
\text { with IPC and placebo }\end{array}$ \\
\hline $\begin{array}{l}\text { (Paixão et al. } \\
\text { 2014) }\end{array}$ & $\begin{array}{l}15 \text { amateur } \\
\text { cyclists }\end{array}$ & Cycling & $4 \times 5: 5 \mathrm{~min}$ & $230 \mathrm{mmHg}$ & Thigh & $12 \mathrm{~min}$ & $\begin{array}{l}3 \text { Wingate tests, } \\
10 \mathrm{~min} \text { rest }\end{array}$ & $\begin{array}{l}\text { Detrimental to max } \mathrm{PO} \\
\text { in } 1^{\text {st }} \text { Wingate. }\end{array}$ \\
\hline $\begin{array}{l}\text { (Paradis- } \\
\text { Deschenes et } \\
\text { al. 2016) }\end{array}$ & $\begin{array}{l}10 \text { strength- } \\
\text { trained }\end{array}$ & $\begin{array}{l}\text { Resistance } \\
\text { exercise }\end{array}$ & $3 \times 5: 5 \mathrm{~min}$ & $200 \mathrm{mmHg}$ & Thigh & $20 \mathrm{~min}$ & $\begin{array}{l}5 \times 5 \text { sets knee } \\
\text { extension } \\
\text { dynamometer }\end{array}$ & $\begin{array}{l}\text { Increase peak force and } \\
\text { muscle } \mathrm{O}_{2} \text { uptake }\end{array}$ \\
\hline
\end{tabular}




\begin{tabular}{|c|c|c|c|c|c|c|c|c|}
\hline $\begin{array}{l}\text { (Patterson et } \\
\text { al. 2015) }\end{array}$ & 14 healthy & Cycling & $4 \times 5: 5 \min$ & $220 \mathrm{mmHg}$ & Thigh & $45 \mathrm{~min}$ & $\begin{array}{l}12 \mathrm{x} 6 \mathrm{~s} \text { sprints, } 30 \mathrm{~s} \\
\text { passive recovery }\end{array}$ & $\begin{array}{l}2-4 \% \text { increase in mean } \\
\text { and peak power output }\end{array}$ \\
\hline $\begin{array}{l}\text { (Sabino- } \\
\text { Carvalho et } \\
\text { al. 2016) }\end{array}$ & 18 runners & Running & $4 \times 5: 5 \mathrm{~min}$ & $220 \mathrm{mmHg}$ & Thigh & $\begin{array}{l}\text { Not } \\
\text { reported }\end{array}$ & $\begin{array}{l}\text { Discontinuous } \\
\text { incremental test to } \\
\text { exhaustion }\end{array}$ & $\begin{array}{l}\text { Increase time to } \\
\text { exhaustion with IPC } \\
\text { and SHAM compared to } \\
\text { CON }\end{array}$ \\
\hline $\begin{array}{l}\text { (Seeger et al. } \\
\text { 2017) }\end{array}$ & 12 healthy & Running & $4 \times 5: 5 \mathrm{~min}$ & $220 \mathrm{mmHg}$ & Thigh & 1 or $24 \mathrm{~h}$ & $5 \mathrm{~km}$ running $\mathrm{TT}$ & $\begin{array}{l}\text { No improvement in } \\
5 \mathrm{~km} \text { running test }\end{array}$ \\
\hline $\begin{array}{l}\text { (Tanaka et } \\
\text { al. 2016) }\end{array}$ & 12 males & $\begin{array}{l}\text { Resistance } \\
\text { exercise }\end{array}$ & $3 \times 5: 5 \mathrm{~min}$ & $>300 \mathrm{mmHg}$ & Thigh & $5 \mathrm{~min}$ & $\begin{array}{l}\text { Knee extension at } \\
20 \% \text { MVC to failure }\end{array}$ & $\begin{array}{l}\text { Enhanced endurance } \\
\text { and accelerated muscle } \\
\text { deoxygenation }\end{array}$ \\
\hline $\begin{array}{l}\text { (Tocco et al. } \\
2015)\end{array}$ & 11 runners & Running & $3 \times 5: 5 \mathrm{~min}$ & $50>\mathrm{SBP}$ & Thigh & $5 \mathrm{~min}$ & $5 \mathrm{~km}$ running $\mathrm{TT}$ & $\begin{array}{l}\text { No improvement in } \\
5 \mathrm{~km} \text { running test }\end{array}$ \\
\hline
\end{tabular}

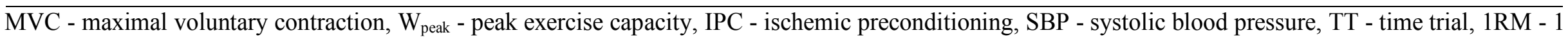
repetition maximum, $\mathrm{CON}$ - control 
Alternatively, to the potential humoral mechanism of adenosine, limb IPC may lead to a local release of adenosine that activates a neural pathway, which subsequently leads to systemic adenosine-receptor activation, implicating a role for adenosine in the neural pathway of limb IPC protection (Liem et al. 2002). The majority of the experimental evidence suggests that limb IPC generates adenosine in the remote tissue, which then activates afferent sensory nerves (Sharma et al. 2015).

Another mechanism explaining limb IPC and athletic performance is the effect of adenosine on the vasculature. Adenosine is a vasodilator that contributes to the vasodilation response during exercise (Joyner \& Proctor 1999). Limb IPC has been shown to increase $\mathrm{O}_{2}$ and blood flow via a vasodilation mediated effect by elevated adenosine levels (Liu et al. 1991). Delivery of $\mathrm{O}_{2}$ to the peripheral tissues by blood is a potential limiting factor during exercise (De Groot et al. 2010); as a result, the vasodilation of blood vessels may overcome this and improve delivery of $\mathrm{O}_{2}$ and clearance of metabolites. However, although the changes in adenosine and $\mathrm{K}_{\text {ATP }}$ channels may enhance maximal exercise-induced blood flow, this does not necessarily improve $\mathrm{O}_{2}$ consumption as $\mathrm{O}_{2}$ extraction can be decreased due to a less efficient flow distribution (Calbet et al. 2006). In contrast, the effects of limb IPC on vasodilators, adenosine and bradykinin, may also contribute to the effects of limb IPC via protection against cellular damage (Riksen et al. 2004) this may assist athletic performance especially when multiple efforts are required within a day or week of competition.

Previous literature suggested the benefit of limb IPC for athletic performance on vascular function, improving muscle blood flow via increases in intramuscular $\mathrm{K}_{\mathrm{ATP}}$ channels in the vascular smooth muscles locally and increased local production of NO due to limb IPC (Horiuchi et al. 2015) as well as from the local release of adenosine (Maczewski et al. 2004) all of which can contribute to vasodilation in muscles (Duncker et al. 1993; Joyner \& Proctor 1999). The vasodilation response after exposure of rat muscles to limb IPC can be blocked using $\mathrm{K}_{\text {ATP }}$ channel blockers (Weselcouch et al. 1993) which suggests activation of $\mathrm{K}_{\text {ATP }}$ channels contributes to the effect of limb IPC on the vasculature (De Groot et al. 2010).

Along with improving muscle blood flow and therefore increasing delivery of $\mathrm{O}_{2}$ to the working muscles, limb IPC initiates functional sympatholysis in humans during exercise (Keller et al. 2004), an important integrative response during exercise to optimally meet the increased metabolic requirements through redistributing blood from inactive areas to active skeletal muscles during exercise (Horiuchi et al. 2015). At the onset of exercise, activation of the sympathetic nervous system results in a systemic vasoconstriction. However, accumulation of metabolites in exercising skeletal muscle locally blunts the sympathetically driven vasoconstriction in the active muscles 
allowing for a marked increase in blood flow to the active muscles (Remensnyder et al. 1962). Horiuchi et al. (2015) showed enhanced functional sympatholysis at rest and at $25 \%$ of maximal voluntary contraction suggesting improved distribution of the regional skeletal muscle blood flow to the most active fibres. This may speed and increase $\mathrm{O}_{2}$ extraction by means of matching $\mathrm{O}_{2}$ demand with $\mathrm{O}_{2}$ delivery or elongating mean capillary transit time as recently suggested by Kido et al. (2015). Additionally, limb IPC may induce up-regulation of the NO pathway (Kharbanda et al. 2001; Loukogeorgakis et al. 2005). As NO antagonises sympathetically mediated vasoconstriction through $\mathrm{K}_{\mathrm{ATP}}$ channel activity (Murphy \& Brayden 1995; Thomas \& Victor 1998), up-regulation of the NO pathway may contribute to the improved functional sympatholysis after limb IPC (Horiuchi et al. 2015b), supporting increased blood flow and $\mathrm{O}_{2}$ delivery to active muscles and contributing to exercise performance.

A reflex pathway may be activated by metabolic changes in the skeletal muscles during limb IPC, which may have a beneficial systemic and/or local effect during subsequent exercise or the metabolic changes in the skeletal muscles induced during limb IPC may positively influence neural pathways to improve performance, through its effect on blood flow locally or systematically (Sharma et al. 2015). For instance, group III and IV afferent sensory neurons respond to mechanical and metabolic alterations in the skeletal muscles during exercise and initiate a reflex that is transmitted to the cardiovascular regulation centers in the brain stem, which in turn increase sympathetic efferent activity and reduces parasympathetic activity during exercise (Murphy et al. 2011). This causes an increase in the heart rate and cardiac output as well as vasoconstriction in non-skeletal muscle vascular beds to divert blood flow to the actively exercising skeletal muscles (Sharma et al. 2015). Limb IPC may cause metabolic changes in the skeletal muscle, activating this neural pathway and thus increasing blood flow and initiating functional sympatholysis. Limb IPC may also lower the sensitivity of the body to fatigue signals, allowing participants to exercise longer enhancing time to exhaustion. Evidence suggests complete skeletal muscle recruitment does not occur during exercise and that regulation of the exercise performance resides in the CNS, which allows the presence of a skeletal muscle recruitment reserve (Noakes 2011). As a result, a desensitising of the afferent groups III and IV potentially allows an increase in neural drive and force output (Gibson et al. 2013). This is reinforced by further literature that proposes limb IPC can cause a greater force generation through augmented neural drive or a reduced perception of fatigue. Limb IPC has been hypothesized to increase work rate in trained cyclists with enhanced muscle recruitment via activation of a proposed muscle recruitment reserve (Crisafulli et al. 2011).

There has been interest in the effect of limb IPC on endothelial function and flow mediated dilation (FMD). Endothelial function is believed to contribute to blood flow regulation (Salvador et al. 2016), increasing tissue oxygenation at rest (Lalonde \& Curnier 2014) to improve vascular 
function and perfusion (Enko et al. 2011; Kraemer et al. 2011) as well as to prevent the decline in vascular function after high intensity exercise (Bailey et al. 2012). Limb IPC mediated increases in muscle blood flow during exercise are likely secondary to the ability of limb IPC to protect against exercise induced and ischemia induced endothelial (Loukogeorgakis et al. 2005; Kharbanda et al. 2002; Bailey et al. 2012) and microvascular dysfunction (Wang et al. 2004).

High intensity exercise has been associated with a significant reduction in artery endotheliumdependent function immediately after cycling (Dawson et al. 2013), however this may be counteracted by the use of limb IPC. For example, results of FMD have shown a change from $5.1 \%$ post control to $3.7 \%$ following $5 \mathrm{~km} \mathrm{TT}$, however following vascular occlusion, FMD was similar post vascular occlusion and post 5km TT; 5.4 and 5.7\% respectively (Bailey et al. 2012). These results highlight that strenuous running is associated with a decrease in endothelial function by $\sim 1.4 \%$. With the use of vascular occlusion this can prevent the exercise-induced decrease in FMD and may provide a strategy to improve endothelial function and therefore improve aerobic exercise performance.

Application of limb IPC to the legs prevents endothelial dysfunction in the upper body, highlighting the systemic effects against IRI beyond the ischemic area (Bailey et al. 2012). These combined effects might improve exercise performance by maintaining the supply of $\mathrm{O}_{2}$ and energy substrates to the skeletal muscles during intense exercise, when the exercising muscle groups might be subject to hypoxia, thereby sustaining contractile activity (Sharma et al. 2015). Previous research has shown that exercise training can improve endothelial function with chronic resistance training protecting against vascular dysfunction in trained athletes, with the addition of limb IPC it can assist with vasodilators (e.g. NO), adenosine and bradykinin that may contribute to the effects of limb IPC via protection against cellular damage (Riksen et al. 2004). Recurring periods of exercise-induced endothelial dysfunction may represent a beneficial stimulus that contributes to longer term improvement of the endothelial function, commonly referred to as hormesis (Padilla et al. 2011), however, limb IPC may provide a strategy to improve endothelial function acutely which may be beneficial prior to athletic performance at competition to increase or maintain $\mathrm{O}_{2}$ and energy substrate supply during intense exercise.

Flow mediated dilation is also related to NO bioavailability and the benefits of limb IPC are related to increased NO synthase (Kido et al. 2015). It is well known that NO is produced from vascular endothelial cells following an increase in shear stress (Kooijman et al. 2008; Green et al. 2014), which is induced by the rapid increase in blood flow, such as that occurs at the onset of exercise or with reperfusion of limb IPC (Kido et al. 2015). Limb IPC is able to improve NO availability (Kimura et al. 2007), in addition to potential vasodilation, NO may impact on the ability to 
consume $\mathrm{O}_{2}$ during exercise as $\mathrm{NO}$ has been shown to attenuate mitochondrial $\mathrm{O}_{2}$ consumption through maintenance of $\mathrm{O}_{2}$ extraction reserve (Cooper \& Brown 2008). Therefore, it was aforementioned that limb IPC could increase blood flow and consequently $\mathrm{O}_{2}$ delivery but there was skepticism whether increased $\mathrm{O}_{2}$ delivery would be utilised by the active skeletal muscle. NO may play a role in the use of limb IPC by increasing $\mathrm{O}_{2}$ extraction to contribute to improved exercise performance.

Research completed by Kido et al. (2015) has identified that limb IPC accelerates the muscular $\mathrm{O}_{2}$ response during systemic whole body exercise, specifically muscle $\mathrm{O}_{2}$ extraction has been analysed through the use of near-infrared spectrometry (NIRS), this reflects the regional balance between $\mathrm{O}_{2}$ utilsation and $\mathrm{O}_{2}$ availability (DeLorey et al. 2003). Prior exercise induced acceleration of the muscle $\mathrm{O}_{2}$ response is associated with mitochondrial enzymatic activation in skeletal muscle (Gandra et al. 2012), this is acutely enhanced by a single bout of exercise (Leek et al. 2001; Fernström et al. 2004) and it may be related to the accelerated $\mathrm{O}_{2}$ metabolism resulting from limb IPC which enhances exercise performance due to skeletal muscle mitochondrial activation, as indicated by accelerated $\mathrm{O}_{2}$ extraction (Kido et al. 2015).

Barbosa et al. (2014) observed deoxygenated hemoglobin and myoglobin concentrations in the forearm were higher at exhaustion point indicating higher $\mathrm{O}_{2}$ extraction after limb IPC. The greater deoxygenated hemoglobin and myoglobin indicates that $\mathrm{O}_{2}$ uptake was proportionally larger than $\mathrm{O}_{2}$ delivery (i.e. greater $\mathrm{O}_{2}$ extraction). Similarly, Kjeld et al. (2014) found lower $\mathrm{O}_{2}$ saturation in the frontal cortex, forearm and thigh at the end of a breath hold after limb IPC of one forearm. However, it cannot be concluded that the greater peak $\mathrm{O}_{2}$ extraction was merely a consequence of longer time to task failure (Barbosa et al. 2014). When compared at equal time points during submaximal exercise, it appears limb IPC does not alter the magnitude of deoxygenation, rather it alters the kinetics, speeding up deoxygenation at the onset of moderate cycling exercise (Kido et al. 2015) which would reduce the $\mathrm{O}_{2}$ deficit (Incognito et al. 2015) and allow increased aerobic energy provision. Such increased aerobic energy provision may reduce the rate of utilisation of the finite anaerobic energy stores, delaying exhaustion (Demarle et al. 2001; Jones et al. 2008; Turnes et al. 2014).

Alternatively, both the cardiovascular and the respiratory systems have been examined and shown to increase during activity following limb IPC, without any improvement in $\mathrm{VO}_{2}$ max but a greater level of physical effort (e.g. Crisafulli et al. 2011). It is speculated that ATP-sparing may occur through a tightening of excitation-contraction coupling, a reduction in futile ion pumping, or through a lower reliance on anaerobic energy production (Pang et al. 1995). If ATP-sparing occurred, it may be expected that muscle efficiency was increased, leading to a larger work per $\mathrm{O}_{2}$ 
consumed (De Groot et al. 2010). Crisafulli et al. (2011) reported that the cascade of metabolic effect induced by the limb IPC strategy could produce a more efficient muscle contraction, thereby promoting an ATP-sparing effect, which in turn, could lead to a larger workload $/ \mathrm{O}_{2}$ consumption and reduce the energy requirements per unit of work done (Crisafulli et al. 2011). It was speculated that limb IPC permits the recruitment of additional higher order motor units via enhanced central motor efferent command, allowing subjects to exercise slightly beyond their individual critical threshold of fatigue for the task (Crisafulli et al. 2011). In practical terms, these effects could be increasing/maintaining the rate of force development during exercise, which would be very important in the context of sprint swimming exercises (Lisbôa et al. 2017).

In vivo studies have shown that limb IPC leads to opening of mitochondrial $\mathrm{K}_{\mathrm{ATP}}$ channels and uncoupling of oxidative phosphorylation (Fryer et al. 2000). As a result, it was proposed that limb IPC allows for faster uptake of acetyl-CoA by mitochondria thus maintaining lactate accumulation at a metabolically acceptable level due to greater contribution of aerobically generated ATP for exercise rather than involvement from the anaerobic capacity (Jean-St-Michel et al. 2011). However, some studies have reported lower PCr, ATP and total adenosine nucelotides in addition to reduced buffer capacity immediately after limb IPC (Salvador et al. 2016); this may impair performance reliant on anaerobic metabolism and highlights the importance of appropriate timing for implementation of limb IPC.

The proposed mechanisms discussed predominately occur as a result of the early phase of limb IPC, however, as reported in the clinical literature, there are two phases that result from limb IPC; an early phase is activated immediately and is no longer evident after 4h (Sack et al. 1993; Burckhartt et al. 1994), a second phase presents $24 \mathrm{~h}$ after the application of the limb IPC stimulus and sustained for at least $48 \mathrm{~h}$ (Loukogeorgakis et al. 2005), in some cases the protective effect may still be seen after $72 \mathrm{~h}$ (Eisen et al. 2004), this has been termed delayed or late preconditioning. Once the early and late effects of limb IPC on the cardiovascular system were demonstrated in clinical experimental settings (Hagar et al. 1991; Yamashita et al. 1998), it would be interesting to determine the ideal window of time after which limb IPC could result in exercise benefits (Marocolo et al. 2016).

Relating to athletic performance, both phases have been shown to be mediated by endogenous substances activating $\mathrm{K}_{\mathrm{ATP}}$ channels (Loukogeorgakis et al. 2005; Randhawa et al. 2015). The early phase affects ion channel permeability and the post translational modification of proteins, with release of autocoids such as adenosine, NO and bradykinin (Koch et al. 2014). Whereas, the later phase appears dependent on gene expression and de novo synthesis of proteins involving endothelial health, hemostasis, immune response and cellular energy metabolism (Koch et al. 
2014). A large part of the genetic response involves mitochondrial proteins, leading to more efficient energy homeostasis (Perez-Pinzon 2004). Unique to late phase of limb IPC is the role of ROS, triggering delayed protection (Kaeffer et al. 1997; Bolli et al. 1997; Sun et al. 1996). NO generated in ischemic tissue during ischemia has been proposed as both a trigger and a mediator in late preconditioning (Bolli et al. 1998; Bolli 2000). The formation of peroxynitrite anion from $\mathrm{NO}$ and free $\mathrm{O}_{2}$ radicals is considered an important upstream event during the triggering of delayed limb IPC (Bolli 2000).

There appears to be different timing of mechanisms resulting from limb IPC and therefore may explain variation in results seen to date on performance as timing for specific exercise (e.g. time to exhaustion, single effort anaerobic and repeated sprint) may be influenced by the early and late phase of protection. Therefore, an appropriate protocol needs to be established to effectively utilise these two distinct phases and the resultant physiological responses to improve performance.

\subsubsection{Summary}

It is plausible that the reduced $\mathrm{O}_{2}$ delivery and elevated metabolic accumulation as a result of decreased venous outflow initiates several mechanisms that contribute to the response seen when combining vascular occlusion with exercise including the hormonal response following high load exercise with vascular occlusion whereas limb IPC increases the release of adenosine having a subsequent effect on vasodilation, blood flow and $\mathrm{O}_{2}$ delivery and extraction. The previous results have highlighted the different applications of vascular occlusion and limb IPC with athletes and support its use as a pre-competition strategy. There is a large variance in protocols regarding percentage of 1RM, volume of sets and repetitions and continuous versus intermittent occlusion when combing vascular occlusion with resistance exercise, therefore, it is hard to draw conclusions on the most suitable or effective protocol to implement. There are further disparities in the protocol applied for limb IPC, with several mechanisms attenuating improvement in performance with factors such as cuff pressure and time between limb IPC and athletic performance that are influencing the results and outcomes of limb IPC and exercise performance. Due to the noninvasive nature and easy application of vascular occlusion or limb IPC, it may be advantageous to use within sport (De Groot et al. 2010), for example limb IPC could be used as an intervention before athletes commence their warm up routine (Gibson et al. 2013). However, little is known about the optimal limb IPC protocol and timing to improve exercise performance at present (De Groot et al. 2010). 


\subsection{USE OF VASCULAR OCCLUSION FOR RECOVERY}

The effect of vascular occlusion as a recovery modality has only recently received attention and potential application in athletic performance. Initial research by Beaven et al. (2012) reported varied results on the effect on performance; beneficial effects were identified on power output and sprint performance immediately and $24 \mathrm{~h}$ post. The three min cycles of occlusion and reperfusion were selected to fulfil the duration threshold criterion that has been reported previously (Van Winkle et al. 1991). This threshold has been suggested to reflect the period of ischemia required to accumulate sufficient localised adenosine concentrations where the adenosine receptors are sufficiently populated to elicit a preconditioning effect (Beaven et al. 2012). Importantly, a total ischemic stimulus of 4-6 min has been shown to be most effective at eliciting a protective effect in human myocardium, regardless of the number of cycles of ischemia and reperfusion (Ghosh et al. 2000). Although the occlusion intervention used was effective in improving specific aspects of subsequent exercise performance, negative effects at different time points and different performance measures were also identified resulting in inconclusive results (Beaven et al. 2012). 
Table 2.4 The use of vascular occlusion as a recovery modality

\begin{tabular}{|c|c|c|c|c|c|c|}
\hline Study (date) & Participants & Intervention & $\begin{array}{l}\text { Control } \\
\text { condition }\end{array}$ & Performance test & Time points & Findings \\
\hline $\begin{array}{l}\text { (Beaven et } \\
\text { al. 2012) }\end{array}$ & $\begin{array}{l}14 \text { healthy male } \\
\& \text { females }\end{array}$ & $\begin{array}{l}220 \mathrm{mmHg} \text { for } \\
2 \times 3: 3 \mathrm{~min}\end{array}$ & $\begin{array}{l}15 \mathrm{mmHg} \text { post } \\
\text { exercise for } \\
2 \times 3: 3 \mathrm{~min}\end{array}$ & $\begin{array}{l}\text { 3x squat jump, 3x } \\
\text { CMJ, } 6 \text { reps leg } \\
\text { press, } 6 x 40 \mathrm{~m} \\
\text { sprints }\end{array}$ & 0 and $24 \mathrm{~h}$ & $\begin{array}{l}\text { Beneficial \& negative } \\
\text { effects performance tests } \\
\text { immediately \& } 24 \mathrm{~h} \text { later }\end{array}$ \\
\hline $\begin{array}{l}\text { (Borne et al. } \\
\text { 2016) }\end{array}$ & $\begin{array}{l}33 \text { sprint cyclists } \\
\text { and middle- } \\
\text { distance runners }\end{array}$ & $\begin{array}{l}90-110 \mathrm{mmHg} \\
\text { for } 24 \mathrm{~min}\end{array}$ & $\begin{array}{l}\text { Supine rest } \\
24 \mathrm{~min}\end{array}$ & 30s Wingate test & $0 \mathrm{~h}$ & $\begin{array}{l}\text { No beneficial effect of } \\
\text { occlusion }\end{array}$ \\
\hline $\begin{array}{l}\text { (Garcia et al. } \\
\text { 2017) }\end{array}$ & $\begin{array}{l}8 \text { male rugby } \\
\text { union players }\end{array}$ & $\begin{array}{l}220 \mathrm{mmHg} \text { for } \\
3 \times 5 \mathrm{~min}, 2 \mathrm{~min} \\
\text { reperfusion }\end{array}$ & $\begin{array}{l}\text { Supine rest } \\
21 \mathrm{~min}\end{array}$ & $\begin{array}{l}\text { Agility test, CMJ, } \\
\text { 30s continuous } \\
\text { jump test }\end{array}$ & $1 \mathrm{~h}$ & $\begin{array}{l}\text { No acute improvement in } \\
\text { recovery }\end{array}$ \\
\hline $\begin{array}{l}\text { (Northey et } \\
\text { al. 2016) }\end{array}$ & $\begin{array}{l}12 \text { strength } \\
\text { trained males }\end{array}$ & $\begin{array}{l}220 \mathrm{mmHg} \text { for } \\
2 \times 3: 3 \mathrm{~min} \& \\
\text { supine rest } \\
33 \mathrm{~min}\end{array}$ & $\begin{array}{l}\text { Supine position } \\
45 \mathrm{~min}\end{array}$ & $\begin{array}{l}\text { Concentric peak } \\
\text { isokinetic torque, } \\
\text { jump height from } \\
\text { SJ \& CMJ }\end{array}$ & $0 \mathrm{~h}, 1 \mathrm{~h}, 24 \mathrm{~h}$ & $\begin{array}{l}\text { No improvement in } \\
\text { recovery }\end{array}$ \\
\hline $\begin{array}{l}\text { (Page et al. } \\
\text { 2017) }\end{array}$ & 16 healthy males & $\begin{array}{l}220 \mathrm{mmHg} \text { for } \\
3 \times 5: 5 \mathrm{~min}\end{array}$ & $\begin{array}{l}20 \mathrm{mmHg} \text { for } \\
3 \times 5: 5 \mathrm{~min}\end{array}$ & CMJ, MIVC & 24,48 and $72 \mathrm{~h}$ & Accelerated recovery \\
\hline
\end{tabular}

CMJ - countermovement jump; SJ - squat jump; MIVC - maximal isometric voluntary contraction. 


\subsubsection{Use of vascular occlusion as a recovery strategy}

To date, five studies have investigated the use of vascular occlusion as a potential recovery strategy (Table 2.4) with two studies reporting a beneficial effect (Beaven et al. 2012; Page et al. 2017), and three studies reporting no effect (Northey et al. 2016; Borne et al. 2016; Garcia et al. 2017). The initial study by Beaven et al. (2012) examined the effect of vascular occlusion on recovery, 14 participants performed a series of exercises inducing muscle damage and performance measures prior to being exposed to a control or vascular occlusion condition (Table 2.4). The results indicated vascular occlusion had a beneficial effect on mean squat jump height immediately after, although there were detrimental effects on mean eccentric peak velocity and peak acceleration in the CMJ and mean eccentric PPO in the squat jump immediately post intervention. The results after $24 \mathrm{~h}$ show a beneficial effect in the CMJ after vascular occlusion, specifically on mean concentric and eccentric peak velocity. The inconclusive results initiated further work in this area, and more specifically, in well trained participants.

However, there is disparity within the research that may be explained by methodological differences in the implementation of vascular occlusion, variance in participant activity levels and variability in the stimulus for EIMD. Neither Borne et al. (2016), Northey et al (2016) or Garcia et al. (2017) found vascular occlusion improved rate of recovery in trained participants. Although differences in participant activity levels existed, the methodologies also varied, for instance, Borne et al. (2016) applied 24 min of continual pressure using elastic wraps versus the intermittent application of pressure for $12 \mathrm{~min}$ in other studies (Beaven et al. 2012; Northey et al. 2016). It is possible that the training status of the individuals may explain the differences between studies. For example, the participants in the study by Page et al. (2017) were recreationally active and therefore maximal isometric voluntary contraction was reduced by $18.5 \%$ following EIMD in the control group. In contrast, the participants in the study by Northey et al. (2016) had a history of strength training which resulted in a smaller loss of force production (4\%), which was likely due to the repeated bout effect (Howatson et al. 2007), suggesting that vascular occlusion was less likely to play a role in the trained group. Moreover, standardised cuff pressures have been used (Table 2.4) irrespective of participant characteristics despite recommendations regarding individualisation of cuff pressures relative to thigh girth and resting blood measurements (Loenneke et al. 2015). Similarly, inconsistencies in the timing of recovery markers (1-72h post occlusion) have occurred between studies.

\subsubsection{Proposed mechanisms for the use of vascular occlusion as a recovery modality}

Recovery after strenuous exercise often relies on alleviating the effects of impaired muscle function and soreness (Cochrane et al. 2013). The most frequently proposed mechanism through 
which strategies may contribute to recovery after high-intensity exercise is by increasing or maintaining local and/or systemic blood flow (Barnett 2006; Bishop et al. 2008). Effective recovery strategies enhance blood flow to the recovering muscles, which is assumed to improve $\mathrm{O}_{2}$ and nutrient delivery, and therefore ATP, PCr and glycogen resynthesis (Byrne et al. 2004), while at the same time transporting lactate and metabolic by-products away from the active muscles to removal sites and non-exercised muscles (Cheung et al. 2003). According to some studies, an increase in local and/or systemic blood flow can be achieved through several parameters such as activation of the skeletal muscle pump and release of endothelium-derived relaxing factors which increase venous return and arterial inflow (Davies et al. 2009; Delis et al. 2000). Garcia et al. (2017) speculated the vascular occlusion would induce improvement due to increased blood flow (i.e., reperfusion) and muscular $\mathrm{O}_{2}$ utilisation for a faster recovery of muscular function. However, blood flow and $\mathrm{O}_{2}$ consumption were not assessed in that study and their mechanistic statements were speculative.

The proposed beneficial effects of vascular occlusion on exercise performance and recovery have been attributed to post occlusion increases in blood flow associated with elevated adenosine levels (Liu et al. 1994) and activation of $\mathrm{K}_{\mathrm{ATP}}$ channels (De Groot et al. 2010), which potentially result in elevated blood flow. Within the clinical literature, as previously discussed in section 2.3, vascular occlusion has been used to protect cardiac and skeletal tissue against IRI (Zhao \& VintenJohansen 2006), this is the damage that occurs when blood supply returns to the tissue after a period of prolonged ischemia, causing metabolic and contractile damage (Wang et al. 2011). The metabolic and contractile damage observed following IRI, is similar to that seen in EIMD, namely increased intracellular $\mathrm{Ca}^{2+}$ concentrations (Warren et al. 1995) and an increase in appearance of muscle proteins in the blood and cytokine markers such as CK, lactate dehydrogenase and interleukin-6 (Wang et al. 2011; Chan et al. 2004). Vascular occlusion has shown to attenuate IRI and thus may potentially be used to speed up the recovery process following EIMD, via increased blood flow due to its effects on activating $K_{\text {ATP }}$ channels (De Groot et al. 2010), elevating adenosine levels (Liu et al. 1994) and/or reducing the inflammatory response (Konstantinov et al. 2004), yet the exact mechanisms are currently unknown. Whilst vascular occlusion may attenuate the functional and metabolic damage associated with EIMD, few studies have examined the efficacy of this intervention on recovery (Page et al. 2017).

Page et al. (2017) investigated physiological response; the data demonstrated occlusion attenuated the increase in $\mathrm{CK}$ as evidenced by reduction at 24 and $48 \mathrm{~h}$ following exercise. The reduction in CK following the application of vascular occlusion suggested cell membrane integrity was maintained to a greater extent than the control group, suggesting a reduction in the inflammatory response to EIMD. A reduction in inflammatory response leads to a reduction in muscle oedema 
and intramuscular pressure, which reduces the stimulation of nociceptors, potentially reducing pain, stiffness and muscle soreness sensations (Howatson \& van Someren 2008), which corresponds to the positive reduction on muscle soreness following EIMD in the research by Page et al. (2017).

Speculatively, the mechanism(s) by which occlusion might lead to reduced soreness/pain may be due to a downregulation of circulating leukocytes (Konstantinov et al. 2004) and/or increased NO which is up-regulated in response to vascular occlusion (Rassaf et al. 2014), and appears to be an important intracellular and intercellular regulator of muscle inflammation and muscle remodeling (Tidball 2005). It should be noted however that a reduced inflammatory response may not always be sought. Whilst this may be beneficial for short term recovery, the inflammatory response is important for adaptations to exercise and thus practitioners should take care as to when they apply different recovery techniques (Page et al. 2017).

Although mechanisms were not the primary focus of the investigation by Beaven et al. (2012), it was reported that ischemia can improve muscle function (Lawson \& Downey 1993) as well as exercise performance and maximal $\mathrm{O}_{2}$ uptake in humans (De Groot et al. 2010). It is possible that hypoxia has an effect on muscle spindle reactivity and the alterations of sensorimotor control may contribute to the observed effects on eccentric measures (Hoshikawa et al. 2010). In contrast, the majority of the beneficial effects of occlusion were observed after $24 \mathrm{~h}$ in the study completed by Beaven et al. (2012), the authors suggested that the increased blood flow due to reperfusion and improved muscular $\mathrm{O}_{2}$ utilisation may have led to a more rapid return of muscle function. However, as stated the physiological mechanisms were not examined in the study and therefore this is not conclusive.

\subsubsection{Summary}

Increasing literature is investigating the use of vascular occlusion implemented as a recovery strategy to improve athletic performance following EIMD. However, within the five studies completed, there are many disparities between the protocol implemented, varying from the cuff pressure used, duration of occlusion and reperfusion, along with participant activity level, training history and timing of and performance tests enforced. All of these factors combined prevent any conclusions to be drawn regarding the use of vascular occlusion for recovery and therefore requires further investigation. 


\subsection{VARIABLES OF LIMB IPC AND VASCULAR OCCLUSION}

The inconsistencies in the current literature on the experimental results of limb IPC and vascular occlusion may be a result of the use of different equipment and/or variation in the protocol and procedures previously utilised. A standard protocol for limb IPC has involved a number of cycles of inflation of a cuff on the arm or leg followed by cuff deflation for a short period of time (Sharma et al. 2014). Examples of previous protocols include 3-4 cycles of cuff inflation and deflation, five min cycles, using a cuff inflation of 220mmHg (Bailey et al. 2012; Clevidence et al. 2012; De Groot et al. 2010; Gibson et al. 2013) or continuous occlusion combined with low intensity exercise. A range of cuff pressures and cuff widths have currently been reported within the literature (Table 2.5).

\subsubsection{Cuff Pressure}

It is common in limb IPC and vascular occlusion protocols for the cuff to be inflated to the same absolute occlusion pressure (mmHg) (Jean-St-Michel et al. 2011; Beaven et al. 2012; Cook et al. 2014). However, a standard occlusion pressure may not reduce blood flow to the same degree in different individuals (Hunt et al. 2016). As such, the level of $\mathrm{O}_{2}$ delivery as well as accumulation and clearance rate of local metabolic by-products are likely to be different between individuals (Takarada et al. 2000; Yasuda et al. 2008; Karabulut et al. 2011). Furthermore, complete arterial occlusion causes greater ratings of perceived exertion compared to partial occlusion and limits the tolerable duration of exercise (Yasuda et al. 2009), reducing the effectiveness of vascular occlusion when combined with exercise (Sumide et al. 2009; Kacin \& Strazar 2011).

Within the systematic review completed by Fahs et al. (2012), it was reported that the majority of studies have utilised a cuff pressure of between $140-240 \mathrm{mmHg}$ for the lower body and 100$160 \mathrm{mmHg}$ for the upper body. However, restrictive cuff pressures in this range may not be appropriate for all individuals. The pressure applied should be high enough to occlude venous return from the muscle but low enough to maintain arterial inflow into the muscle (Loenneke et al. 2014). The optimal pressure for beneficial effects on skeletal muscle are currently disputed; however, preliminary data has suggested that there may be a point where greater pressure may not augment the response (e.g. metabolic accumulation) but may actually result in decrements (e.g. decrease muscle activation and fatigue) (Loenneke et al. 2014), the latter more important when applying vascular occlusion with exercise.

Therefore, applying the same pressure to every individual could inadvertently lead to the complete occlusion of blood flow for some participants (Jessee et al. 2016). Figure 2.5 from Jessee et al. (2016) highlights the effect of applying an absolute pressure of $160 \mathrm{mmHg}$, resulting in arterial 
occlusion at rest for many participants in the upper body [also true in lower body (Loenneke et al. 2013)]. This same pressure may not necessarily restrict the same amount of blood flow in all individuals or under conditions in which a different vascular occlusion device is used, since the amount of tissue surrounding the blood vessels influences the pressure exerted on the vasculature and therefore, the degree of occlusion (Hargens et al. 1987). Consequently, the stimulus and response may differ depending on the degree of occlusion in each individual. To control for this, efforts to make restriction relative are made by applying a pressure $50 \mathrm{mmHg}$ greater than systolic blood pressure or $20 \mathrm{mmHg}$ less than brachial systolic blood pressure (Horiuchi 2017; Pope et al. 2013). Although this makes it relative, the resulting pressure may vary as a percentage of arterial occlusion due to differences in cuff width/type (Jessee et al. 2016) and furthermore no guidance whether pressure should be above or below and to what degree greater than systolic blood pressure. Thus, pressure should also be made relative to the individual and cuff being used during the actual occlusion session.

It is often speculated in the literature that thigh circumference or composition of the limb may restrict flow differently between individuals which might account for some of the variability in the response to vascular occlusion combined with exercise (Karabulut et al. 2011). Karabulut \& Perez (2013) proposed that variation in the regional body composition between genders could change the degree of pressure caused by the tightness of the cuff used during occlusion and the subsequent effect on neuromuscular activation. Thicker skin and subcutaneous fat in the thighs may act as a barrier between the occlusion cuffs and circulatory pathways resulting in low or no change in the diameter of the targeted blood vessels minimising overall effect of external pressure. Karabulut $\&$ Perez (2013) completed a study to investigate the effects of this; participants were required to have their upper leg length, thigh circumference and skin and subcutaneous fat thickness determined. Following this an elastic cuff was placed proximal to the inguinal area of the right thigh and was manually tightened to the final pressure which was determined by multiplying arm systolic blood pressure by 1.44. Participants lifted the same weight (set between 41 and $68 \mathrm{Nm}$ ) during dynamic exercise for both testing sessions. The study reported that skin and subcutaneous fat thickness was the most important independent variable creating variations in the amount of pressure being applied by the tightness of the occlusion cuff. The changes in EMG response due to tightness of cuff and the effect of skin, and subcutaneous fat thickness on tightness of cuff provide important details on the occlusion protocol and leg composition on NMF during exercise with vascular occlusion (Karabulut \& Perez 2013). It is likely the degree of intramuscular pressure depends on the architecture features of the muscle (Hunt et al. 2016). Hypertrophied muscles have a greater thickness and volume, and subsequently blood flow occlusion may occur at relatively lower pressures (Wernbom et al. 2006). Despite this, fat as opposed to muscle cross-sectional area 
has explained most of the variance in the cuff pressure required to completely occlude arterial flow (Loenneke \& Abe 2012).

However, hierarchical regression models have been used to determine what factors (i.e., gender, leg size, blood pressure, and limb composition) should be accounted for when prescribing the cuff pressure for this training technique (Loenneke et al. 2012). An interesting finding was that the leg circumference (field method) models predicted the cuff pressure needed to restrict arterial blood flow equally or better than the limb composition (lab method) models. This provided evidence that measuring limb circumference rather than limb composition would be adequate for determining restrictive cuff pressure (Loenneke et al. 2012).

Consequently, Loenneke et al. (2013) further investigated occlusion pressures that were relative to limb circumference. Thigh circumference was measured $33 \%$ distal to the inguinal crease due to providing an accurate representation of the site at which the cuffs would be applied. Thigh circumference was plotted with arterial occlusion pressure (AOP) for each individual. For the occlusion protocol, an inflation pressure of $60 \%$ of participants predicted AOP was used to ensure that the inflated cuff pressure would not cause arterial occlusion. The following measurements and occlusion pressure was determined; $<45-50 \mathrm{~cm}=120 \mathrm{mmHg} ; 51-55 \mathrm{~cm}=150 \mathrm{mmHg} ; 56-59 \mathrm{~cm}$ $=180 \mathrm{mmHg}$ and $\geq 60 \mathrm{~cm}=210 \mathrm{mmHg}$. However, all of these results have only been tested within the lower body and therefore may not translate to occlusion for the upper body and published research has not yet examined the efficacy of these recommendations for enhancing adaptive responses to training (Scott et al. 2015).

To account for inter-individual differences, some investigators have implemented occlusion as a percentage of estimated AOP (Scott et al. 2015). The pressure can be made relative to an individual's AOP, which is the minimum pressure required to completely occlude blood flow. By setting the pressure at a percentage of the pressure required to completely occlude blood flow with a specific cuff, it should allow a stimulus that accounts for differences in cuff type and limb size (Jessee et al. 2016). Within the literature the use of a hand-held bidirectional Doppler probe placed on the posterior tibial artery for detection of complete arterial occlusion has been reported, to subsequently calculate percentage of pressure required to cause partial arterial occlusion (e.g. Laurentino et al. 2008).

Within this protocol, a nylon cuff $(17.5 \times 90 \mathrm{~cm})$ was placed at the top of the thigh and inflated to the lowest point at which an auscultatory pulse was no longer detected. This value was defined as the AOP (Teixeira et al. 2017). When arterial flow was no longer detected, cuff pressure was decreased by $100 \mathrm{mmHg}$ until arterial flow was present. This process has also been reported for 
wide $(13.5 \mathrm{~cm})$ and narrow $(5 \mathrm{~cm})$ cuffs (Loenneke et al. 2012); the results highlighted that restrictive cuff pressure should be based on width of the cuff; the wider cuffs cut off arterial flow at a lower pressure compared to the narrow cuffs. Reports suggest that an arbitrary pressure of $100 \mathrm{mmHg}$ with a $13.5 \mathrm{~cm}$ cuff was similar to a pressure of $210 \mathrm{mmHg}$ with a $5 \mathrm{~cm}$ cuff (Loenneke et al. 2013). By measuring the AOP with the cuff actually used during the occlusion protocol, then applying the stimulus as a percentage of that value, this ensures that the methodology is replicable, and allows investigators to compare various combinations of load and relative restriction pressures (Loenneke et al. 2013).

Due to the increasingly common use of Doppler ultrasound to determine occlusion pressure Bezerra de Morais et al. (2017) examined the reproducibility of Doppler ultrasound for the measurement of occlusion pressure in upper limbs and determined the predictive variables. Thirteen male volunteers attended three times to the laboratory. On the first day, arm circumference, systolic and diastolic blood pressure, and brachial artery occlusion pressure were assessed. On second and third days, only occlusion pressure was assessed. A coefficient of variation $(\mathrm{CV})$ of $5.6 \%$ and an intra-class correlation coefficient (ICC) of 0.795 for the Doppler was found. In the analysis of occlusion pressure predictive variables, the Spearman coefficients were 0.813 systolic blood pressure, 0.786 diastolic blood pressure and 0.388 arm circumference. Therefore, these results support that Doppler has good reproducibility for occlusion pressure and that systolic and diastolic blood pressure should be considered to determine occlusion pressure in upper limbs. 


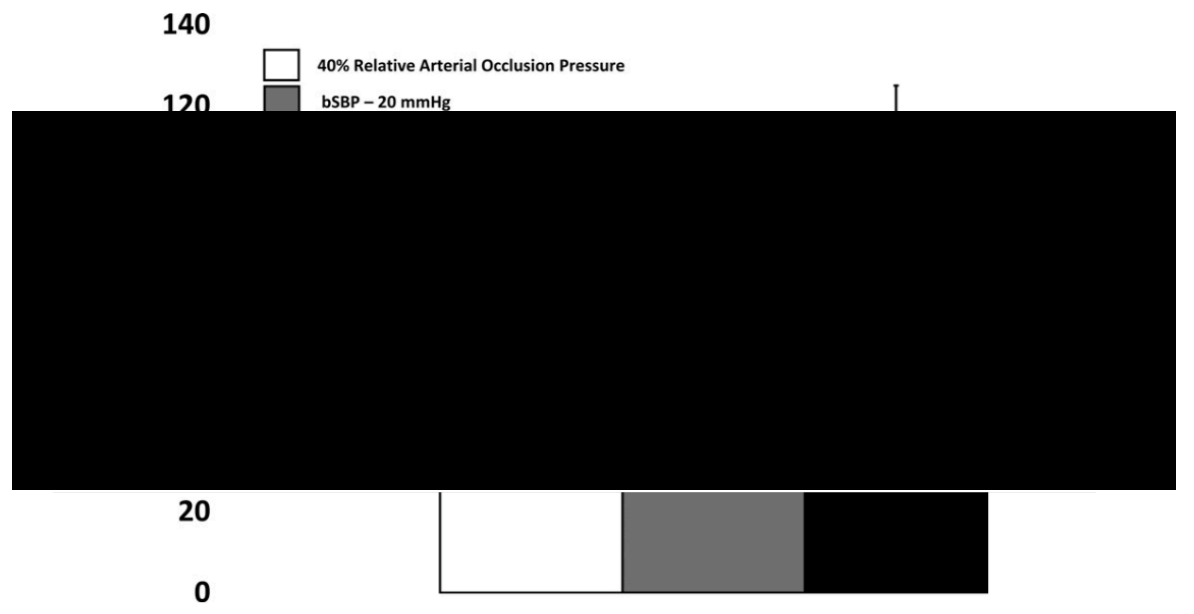

Figure 2.5 The percentage of arterial occlusion applied when using vascular occlusion with absolute and relative pressures (bSBP - brachial systolic blood pressure). Data from Loenneke et al. (2015), extracted from Jessee et al. (2016). 
Previous debate exists whether systolic blood pressure should even be considered since studies report a moderate $(r=0.56$, Younger et al. 2004) if not absent $[r=0.05$, Crenshaw et al. 1988; Loenneke et al. 2012) relationship between systolic blood pressure and limb occlusion pressure. This suggests that basing cuff pressure on systolic blood pressure alone may not lead to optimal occlusion and further variables should be investigated (Hunt et al. 2016). Loenneke et al. (2015) provided evidence regarding the use of systolic blood pressure as a predictor but extended the research, investigating the impact of cuff pressure on upper and lower limbs, identifying optimum individual cuff pressure. Within this study 171 participants were investigated; male and female un-trained participants (reported absence of cardiovascular or metabolic disease as criteria). Following baseline measures (blood pressure, arm and thigh circumference, muscle and fat thickness) AOP was determined using a Doppler ultrasound. For the upper body two different models of hierarchal linear regression were used to determine which variables predicted the pressure at which arterial occlusion occurred. Models included arm circumference or composition and systolic and diastolic blood pressure. Similar was repeated for the lower body; thigh circumference, systolic and diastolic were added into the model.

Results from this study identified that arm circumference or tissue composition may need to be accounted for when using vascular occlusion (Loenneke et al., 2015). However, absolute arm size may be more important than composition. Similarly, thigh circumference was reported as the biggest predictor of arterial occlusion in the lower body, highlighting that pressure in the lower body should be individualised on the basis of thigh size (larger thigh requires greater pressure). The results highlighted that for both males and female's thigh girth and arterial blood pressure are important determinants for vascular occlusion. Limitations of the study that should be considered, all measurements were taken at rest in the supine position and therefore research is required to identify if the same results are identified during exercise when in the upright position. Furthermore, little evidence has determined what percentage of arterial occlusion is required to achieve complete venous occlusion, which is the primary aim of vascular occlusion implementation for training, pre-competition or recovery strategies.

This research supported previous findings in which thigh circumference was the biggest predictor of arterial occlusion in the lower body (Loenneke et al. 2012). This reinforces the idea that pressures in the lower body should to some degree be individualised on the basis of thigh size, meaning bigger thighs require greater pressures and smaller thighs require less pressure (Loenneke et al. 2015). When the regression models were separated by sex, circumference was still explaining the most variance in arterial occlusion. When applying occlusion to the upper body, results suggest brachial systolic blood pressure and arm circumference should be accounted for when setting the pressure. The fact that brachial systolic blood pressure explains a significant amount of the 
variance with arterial occlusion is logical given that they are essentially measures of the same thing (Loenneke et al. 2015). As a result equations were reported by Loenneke et al. (2015) to determine upper (2.1) and lower (2.2) body occlusion pressure based on blood pressure and arm or thigh circumference, respectively. This method of determining individual cuff pressure in the absence of a Doppler ultrasound is practical to use in the field.

Respective equations (Loenneke et al., 2015):

(2.1) Arterial occlusion $(\mathrm{mmHg})=0.514(\mathrm{SBP})+0.339(\mathrm{DBP})+1.461($ arm circumference $)$ $+17.236$

(2.2) Arterial occlusion $(\mathrm{mmHg})=5.893($ thigh circumference $)+0.734(\mathrm{DBP})+0.912(\mathrm{SBP})$ $-220.046$

Where $\mathrm{SBP}=$ systolic blood pressure; $\mathrm{DBP}=$ diastolic blood pressure

Laurentino et al. (2008) determined the pressure required for complete vascular restriction at the upper thigh during rest, and subsequently employed an occlusion pressure of $80 \%$ AOP during low-load resistance training. However further results have demonstrated that an individualised cuff pressure of $50 \%$ estimated AOP appears to maximise EMG amplitude and increase acute decrements in torque during and following low-load knee extension exercise (Loenneke et al. 2015). This is supported by research suggesting increased restrictive pressures (60\% estimated AOP) did not result in further augmentation of these responses (Scott et al. 2015) and Mattocks et al. (2017) indicated higher RPE ratings and discomfort and lower volume completed at $90 \%$ compared to $50 \% \mathrm{AOP}$ and below.

Further research by Fatela et al. (2016) examined fourteen men exercising at $20 \% 1 \mathrm{RM}$ combined with 40, 60 and $80 \%$ AOP. Restrictive pressures were calculated based on direct blood-flow measurements taken at rest on each participant in accordance with previous Doppler measures. Results demonstrated muscular activation, as well as NMF, varies as a function of relative occlusion. This extends previous statements and demonstrating the individual determination of vascular restriction levels is crucial before engaging in occlusion exercise. In addition to the emphasis on individualisation of cuff pressure this study also demonstrated that muscle fatigue is dependent on the relative level of vascular restriction which is novel within the literature and highlights the protocol still requires further investigation. The rapid decrease in peak velocity at pressures above $70 \%$ align previous findings, in which pressures above $60 \%$ would theoretically affect both the arterial and the venous circulation (Mouser et al. 2017). Due to the decrease in 
transmitted pressure toward the centre of the limb, it would be expected that pressures greater than $60 \%$ of arterial occlusion are required before the arteries become compressed. Once compressed, blood velocity decreases rapidly, likely due to the decreased amount of time that the artery remains open during systole (Mouser et al. 2017).

Scott et al. (2015) questioned if equivalent restrictive pressures are used for both the arms and the legs, reporting it is likely that either arterial inflow in the arms will be limited or that insufficient venous occlusion for venous pooling will occur in the legs. Based on previous research where limb circumference explained most of the variance in the cuff pressure required to occlude arterial flow and may influence the fatigue response during occlusion, a different cuff pressure would be required to elicit optimal occlusion of upper and lower limbs (Hunt et al. 2016). Research completed by Sharma et al. (2014) tested a range of cuff inflation pressures (140, 160 and $180 \mathrm{mmHg}$ ) to induce limb ischemia in upper and lower limbs; results identified that ischemia can be induced in the upper limb at a much lower cuff inflation pressure compared with $200 \mathrm{mmHg}$ that is generally used for limb IPC, provided cuff inflation pressure is $\sim 30 \mathrm{mmHg}$ greater than resting systolic blood pressure. It was also proposed that to induce occlusion in the lower limb, a cuff pressure of $\sim 55 \mathrm{mmHg}$ greater than resting systolic blood pressure is required to induce ischemia; $140 \mathrm{mmHg}$ did not occlude and $160 \mathrm{mmHg}$ cuff pressure only occluded in one volunteer. In the upper limb, cuff inflation of 160 and $180 \mathrm{mmHg}$ caused a decrease in $\mathrm{pH}$ of capillary arterial blood, whereas there was no change in $\mathrm{pH}$ at any cuff pressure in the lower limb. The results highlighted different physiological responses and tolerability of limb IPC based on the limb and blood pressure cuff inflation used (Sharma et al. 2014). Furthermore, Hunt et al. (2016) determined the level of blood flow restriction at different external cuff pressures applied to upper and lower limbs. With wide cuff $(13 \mathrm{~cm})$ application, the external pressure required to illicit $60 \%$ occlusion in the popliteal was $111 \pm 12 \mathrm{mmHg}$ and was greater than in the brachial at $101 \pm 12 \mathrm{mmHg}$. Mean arterial pressure and adipose thickness were the largest independent determinants of lower and upper body partial occlusion (60\% AOP) pressures, respectively. Despite entering further variables into an upper (diastolic blood pressure and body mass index) and lower (diastolic blood pressure and adipose thickness) limb regression model, the majority of variance remains unexplained.

Increased attention has been provided to the influence of cuff pressure within the last year (20162017), especially regarding occlusion pressure applied during exercise. Methods of individualising cuff pressure have been acknowledged and initial research into factors affecting pressure and percentage occlusion required are appearing within the literature, for instance cuff pressure above $60 \%$ AOP does not seem to offer any additional advantage. However, variance amongst Doppler regarding protocols exist; in some instances, research has completed 
determination of AOP whilst supine, whereas others during standing. Furthermore, there is a disparity between load (20 or 30\% 1RM) and upper and lower body. Due to inconsistency in the research, there is still no definitive protocol to apply. In the instance Doppler is unavailable within the field in elite sport, the method described by Loenneke et al. (2015) which differentiates between upper and lower body may provide a good alternative. The efficacy and application in elite sport is yet to be determined. Limbs with a larger circumference require higher occlusive pressures to reach the same level of arterial occlusion. Furthermore, limb circumference was, if not more effective than laboratory-based measures of limb composition in predicting the pressure required to restrict arterial blood flow (Loenneke et al. 2012). The majority of research into vascular occlusion pressure when being combined with exercise, is moving towards individualised cuff pressure, however, no attempt has been established to individualise when limb IPC is applied at passive rest, other than use of systolic blood pressure, considerations should also be made regarding the impact of perception, pain and discomfort if high pressures are applied in individuals with smaller thigh circumference.

\subsubsection{Cuff Width}

Vascular occlusion in the research literature is typically accomplished via inflation of a pneumatic cuff placed at the proximal portion of the limb; this is further modified by differing inflation pressures as discussed and cuff widths (Mouser et al. 2017). Many studies utilising occlusion have applied a single arbitrary pressure to all participants and not accounted for cuff width (Table 2.5).

Currently, wide and narrow, non-elastic and elastic cuffs are used in the research (Rossow et al. 2012). The size of the cuff, especially the width, has been variable within the literature despite it being an important variable to consider as wider cuffs have been shown to be more effective in restricting blood flow at lower inflation pressures compared to narrower restrictive cuffs (Loenneke et al. 2012). Many studies have used KAATSU master elastic restrictive cuffs, which are typically $3 \mathrm{~cm}$ wide for upper body and $5 \mathrm{~cm}$ for lower body. Other studies have used a variety of cuffs ranging in width from $3 \mathrm{~cm}$ to $18.5 \mathrm{~cm}$ dependent on material and manufacturing make used. However, many of the studies within the literature do not report the width of the cuff used (Fahs et al. 2012), this is problematic as one determinant of the amount of occlusion is dependent upon the size of the cuff used (Loenneke et al. 2015). As cuff width increases, the pressure requirements to achieve a given percentage of restricted blood flow decreases (Loenneke et al. 2012). For example, applying $200 \mathrm{mmHg}$ across $5 \mathrm{~cm}$ with a KAATSU master cuff will likely produce a different stimulus than $200 \mathrm{mmHg}$ applied over $13.5 \mathrm{~cm}$ with a different cuff.

It has been noted that if using wider restrictive cuffs for vascular occlusion in combination with elevated systolic cuff pressures, the exercise bout may become uncomfortable and the exercise 
volume may be limited (Fahs et al. 2012). For example, high levels of pain in the quadriceps have been reported during acute occlusion during knee extensor exercise using a cuff $13.5 \mathrm{~cm}$ wide inflated to $200 \mathrm{mmHg}$ during exercise (Wernbom et al. 2006). When directly comparing the effect of cuff width, it has been identified that the perceptual response to occlusion knee extension exercise with wider $(13.5 \mathrm{~cm})$ cuffs causes greater ratings of pain and perceived exertion during exercise and limits the exercise volume compared to narrow $(5 \mathrm{~cm})$ cuffs inflated to the same pressure (Rossow et al. 2012). In addition to increased perception, the wide cuffs caused a greater elevation in heart rate, brachial and central blood pressure (Rossow et al. 2012) therefore this should be a consideration during study design. There also needs to be distinguished protocols between lower limb and upper limbs. For the upper body, relatively narrow cuffs $(3-3.5 \mathrm{~cm})$ appear to be preferable. Additionally, narrow cuffs may be beneficial as wider cuffs can restrict the full range of motion during exercise (Loenneke et al. 2015).

Physiologically, Fahs et al. (2012) indicated a wider $(18.5 \mathrm{~cm})$ cuff, set to pressures of 150 and $200 \mathrm{mmHg}$, elevated intramuscular inorganic Pi, however, when a narrow cuff $(5 \mathrm{~cm})$ was applied, although with slightly higher restrictive pressures $(230$ vs. $180 \mathrm{mmHg})$, emanated more intramuscular inorganic Pi during exercise. This suggests to increase fatigue and motor unit recruitment with low loads during lower body vascular occlusion exercise, slightly higher restrictive cuff pressure may be optimal with narrow cuffs while lower restrictive cuff pressures may be sufficient with wider cuffs (Fahs et al. 2012).

Two recent studies have investigated the effect of cuff width on required inflation pressure in the upper body. Jessee et al. (2016) examined differences in upper arm AOP between three different cuff widths and how individual characteristics influenced this. Differences in AOP were observed between cuff widths $(\mathrm{p}<0.001)$. The 5 -cm-wide cuff required the greatest inflation pressure (145 $\pm 19 \mathrm{mmHg})$, followed by the $10 \mathrm{~cm}(123 \pm 13 \mathrm{mmHg})$, and 12 -cm-wide cuff $(120 \pm 12 \mathrm{mmHg})$. A model encompassing arm circumference, arm length, arterial and brachial blood pressure and sex explained the most variance in AOP cuff pressure. However, arm circumference explained the most unique variance for each cuff. It was concluded that the AOP is dependent upon cuff width, highlighting the need for authors to report cuff width and consider the impact it has on restriction with a key point highlighted that an inverse relationship exists between cuff width and AOP in the upper body (Jessee et al. 2016).

Consistent, Mouser et al. (2017) identified an inverse relationship between systolic pressure and cuff width for both men $(5 \mathrm{~cm}: 160 \pm 16 \mathrm{mmHg} ; 10 \mathrm{~cm}: 125 \pm 8 ; 12 \mathrm{~cm}: 122 \pm 9, \mathrm{p}<0.001)$ and women (5 cm: $139 \pm 18 \mathrm{mmHg} ; 10 \mathrm{~cm}: 114 \pm 11 ; 12 \mathrm{~cm}: 110 \pm 10, \mathrm{p}<0.001)$. For the diastolic pressure measurement, there was also an inverse relationship with cuff width for both men $(5 \mathrm{~cm}$ : 
$104 \pm 13 \mathrm{mmHg} ; 10 \mathrm{~cm}: 73 \pm 8 ; 12 \mathrm{~cm}: 68 \pm 9, \mathrm{p}<0.001)$ and women $(5 \mathrm{~cm}: 89 \pm 15 \mathrm{mmHg} ; 10$ $\mathrm{cm}: 67 \pm 10 ; 12 \mathrm{~cm}: 63 \pm 11, \mathrm{p}<0.001)$. Following an examination of relative pressures and their relationship to the measured diastolic blood pressure with each cuff, it was reported lower restriction pressures only affect the venous system indicating cuff pressures that are below the diastolic pressure will affect venous return without compressing the arteries (Mouser et al. 2017).

However, the majority of this research, especially recently, has been within the upper body and therefore there is a lack of research specifically for the effect and recommendations of cuff width on occlusion pressure in the lower limbs. Currently, no optimal cuff width/pressure combination has been established for vascular occlusion and limb IPC; therefore, no standard exists regarding the application of occlusion. As such, potential issues arise in finding an optimal restriction pressure due to the numerous different cuff widths and pressures used (Table 2.5). Despite this, it is important to consider the effect of the cuff width on pressure applied and percentage AOP. In addition to cuff pressure and width, the following sections will outline other influencing factors.

\subsubsection{Cuff Material}

Within the literature there has been a gradual move towards application of relative as opposed to absolute restrictive pressures and acknowledgement of the impact of cuff pressure. However, within the literature, not all methodological concerns have been addressed (Buckner et al. 2017). Specifically, depending on the device, cuffs are made from different materials. For example, a variety of devices have been used to restrict blood flow during exercise, including elastic knee wraps, elastic belts with a pneumatic bag inside, nylon pneumatic cuffs, or a traditional nylon blood pressure cuff (Loenneke et al. 2012).

While elastic automated cuff systems have been developed, it may be more practical to employ inflatable cuffs or simple elastic wraps, particularly when large groups are performing vascular occlusion (Scott et al. 2015). The use of elastic knee wraps in particular has been recently popularised in research and real-world contexts as a practical method for implementing vascular occlusion without the need for expensive specialised equipment (Loenneke \& Pujol 2009). Loenneke et al. (2014) examined differences in cuff type (nylon vs. elastic) in the lower body, finding that there were no differences in the repetitions to fatigue or perceptual response between different type of cuffs, suggesting that the occlusion stimulus was relatively similar. This is supported by Dankel et al. (2017) reporting no difference between EMG amplitude or post-exercise strength measures when a $3 \mathrm{~cm}$ elastic cuff $(160 \mathrm{mmHg})$ was compared to $5 \mathrm{~cm}$ nylon cuff $(40 \%$ individuals AOP), the only difference identified was lower levels of discomfort with the nylon cuff. Whereas, Buckner et al. (2017) reported resting AOP was different between nylon (139 \pm $14 \mathrm{mmHg})$ and elastic $(246 \pm 71 \mathrm{mmHg}, \mathrm{p}<0.001)$ cuffs, however, when exercising at $40 \%$ of each 
cuff's respective AOP (nylon: $57 \pm 7$ vs. elastic: $106 \pm 38 \mathrm{mmHg}, \mathrm{p}<0.001$ ), there were no differences in repetitions to failure, torque, muscle thickness, or muscle activation between the cuffs. Therefore, it was concluded exercising with cuffs of different material but similar width resulted in the same acute muscular response when the cuffs were inflated to a pressure relative to each individual cuff. Furthermore, of importance to highlight, differences in resting AOP were observed despite similar-sized cuffs. This demonstrates the importance of measuring AOP before applying the vascular occlusion stimulus, for example, if an arbitrary pressure of $200 \mathrm{mmHg}$ were applied with each cuff, the majority of individuals in the study using the nylon cuff would be fully occluded, whereas, the elastic cuff condition would be receiving, on average, a stimulus of approximately $80 \%$ AOP. These differences in AOP are contrary to the findings of Loenneke et al. (2014) who observed no differences in AOP between cuff types (nylon vs. elastic) in the lower body. As such, anatomical differences between the upper and lower body may explain some of this discrepancy (Buckner et al. 2017).

\subsubsection{Continuous vs intermittent occlusion}

Occlusion with resistance exercise has previously been completed with either continuous or intermittent blood flow restriction during the exercise training (Fahs et al. 2012). During intermittent vascular occlusion with exercise, muscle fatigue can quickly be reduced during rest periods between exercise sets, similar to free flow exercise. During resistance exercise some blood flow restriction occurs due to the muscle contraction. Therefore, external blood flow restriction applied only during the exercise period may not provide an additional stimulus (i.e. induce muscle fatigue) to cause muscular adaptations. In fact, during low-load resistance exercise, continuous blood flow restriction has been shown to be more effective in causing muscle fatigue (Cook et al. 2007) and metabolic stress (Suga et al. 2012) compared to intermittent blood flow restriction.

An alternative to continuous occlusion training is to deflate the cuff during rest periods, otherwise known as intermittent occlusion. Similarly, intermittent occlusion has been shown to increase muscle strength (Evans et al. 2010) and size (Kacin \& Strazar 2011) over no vascular occlusion. Although both continuous and intermittent vascular occlusion training appear to increase skeletal muscle size and strength at low loads, the efficacy of intermittent and continuous occlusion training have received limited attention (Fitschen et al. 2014). 
Table 2.5: Summary of restrictive cuff pressures, cuff widths and times between vascular occlusion/limb ischemic preconditioning and performance (2015-2017 \& excluding reviews)

\begin{tabular}{|c|c|c|c|}
\hline Author & $\begin{array}{l}\text { Time between occlusion } \\
\text { \& performance (min) }\end{array}$ & $\begin{array}{l}\text { Restrictive cuff } \\
\text { pressure (mmHg) }\end{array}$ & $\begin{array}{l}\text { Restrictive cuff } \\
\text { width }(\mathrm{cm})\end{array}$ \\
\hline (Barbosa et al. 2015) & 25 & 200 & $\mathrm{X}$ \\
\hline (Cocking et al. 2017) & 20 & 220 & 13.5 \\
\hline (Cruz et al. 2015) & 90 & 220 & $\mathrm{X}$ \\
\hline (Dankel et al. 2017) & Applied during $3 \mathrm{~min}$ rest & $70 \%$ AOP & 5 \\
\hline (Fatela et al. 2016) & $\mathrm{X}$ & $40,60,80 \%$ AOP & 13 \\
\hline $\begin{array}{l}\text { (Hittinger et al. } \\
\text { 2015) }\end{array}$ & 45 & $10-20>$ SBP & $\mathrm{X}$ \\
\hline $\begin{array}{l}\text { (Husmann et al. } \\
\text { 2017) }\end{array}$ & During $5 \mathrm{~min}$ exercise & $60 \%$ AOP & 10 \\
\hline (Kido et al. 2015) & 5 & $>300$ & $\mathrm{X}$ \\
\hline (Kraus et al. 2015) & 15 & $\mathrm{X}$ & $\mathrm{X}$ \\
\hline (Lauver et al. 2017) & During exercise & $130 \% \mathrm{SBP}$ & 6 \\
\hline (Lisbôa et al. 2017) & $60,120 \& 480$ & 220 & $\mathrm{X}$ \\
\hline $\begin{array}{l}\text { (Marocolo et al. } \\
\text { 2015) }\end{array}$ & 15 & 200 & $\mathrm{X}$ \\
\hline $\begin{array}{l}\text { (Marocolo et al. } \\
\text { 2016) }\end{array}$ & 8 & 200 & 13 \\
\hline $\begin{array}{l}\text { (Paradis-Deschenes } \\
\text { et al. 2016) }\end{array}$ & 20 & 200 & $\mathrm{X}$ \\
\hline $\begin{array}{l}\text { (Patterson et al. } \\
\text { 2015) }\end{array}$ & 45 & 220 & 14.5 \\
\hline $\begin{array}{l}\text { (Sabino-Carvalho et } \\
\text { al. 2016) }\end{array}$ & 10 & 220 & 13.5 \\
\hline (Seeger et al. 2017) & $60,24(\mathrm{~h})$ & 220 & $\mathrm{X}$ \\
\hline (Tanaka et al. 2016) & 5 & $>300$ & $\mathrm{X}$ \\
\hline (Teixeira et al. 2017) & During exercise & $80 \%$ AOP & 17.5 \\
\hline (Tocco et al. 2015) & 5 & $50>\mathrm{SBP}$ & $\mathrm{X}$ \\
\hline Average & $22 \min$ (exclude $24 \mathrm{~h}$ ) & & 11.8 \\
\hline Range & $5 \min -24 h$ & & $5-17.5$ \\
\hline
\end{tabular}

$\mathrm{AOP}$ - arterial occlusion pressure; SBP - systolic blood pressure 
Previous research has shown knee extension exercise with intermittent blood flow restriction only produced a 3.4\% increase in mid-thigh knee extensor cross sectional area and no significant increases in strength (Kacin \& Strazar 2011). In contrast, another study observed an 18\% increase in force during a maximal voluntary contraction of the triceps surae after 4-weeks of training with intermittent blood flow restriction (Evans et al. 2010). The latter study did not observe any increase in strength in the contralateral limb trained without any blood flow restriction suggesting that even intermittent blood flow restriction may augment strength adaptations.

Fitschen et al. (2014) performed two studies to compare intermittent and continuous occlusion exercise. In study one, eleven participants randomly proceeded through three treatments of unilateral leg extensions to failure: (i) continuous occlusion, (ii) intermittent occlusion and (iii) control (exercise without occlusion). Continuous occlusion resulted in significantly greater pain than intermittent occlusion or control $(\mathrm{p}<0.05)$, yet pain did not significantly differ between intermittent occlusion exercise and the control condition during any set. Both occlusion conditions resulted in significantly fewer repetitions to failure than control. This suggests that an acute bout of intermittent occlusion exercise may produce as much muscle fatigue as an acute bout of continuous occlusion exercise, but with less pain. This is supported by a previous study comparing pain during continuous occlusion exercise with normal low-load exercise finding that participants experienced significantly more pain during continuous occlusion exercise (Fitschen et al. 2014). This suggests intermittent occlusion exercise may produce similar fatigue as continuous occlusion exercise, but with significantly less pain (Fitschen et al. 2014).

\subsubsection{Limb IPC dose}

The limb IPC procedures applied in the majority of literature relating to athletic performance mimicked that used by De Groot et al. (2010). This process involved rendering the limb ischemic for five min, followed by five min of intermittent reperfusion and was based on previously published studies assessing the effects of brief cycles of limb ischemia-reperfusion in human participants on myocardial injury and endothelial function (e.g. Hausenloy \& Yellon 2008). Total occlusion periods range from $6 \mathrm{~min}$ up to $>30 \mathrm{~min}$, with complete limb IPC and vascular occlusion protocol lasting up to $40 \mathrm{~min}$ (Figure 2.6) (Marocolo et al. 2016). Specifically for limb IPC and vascular occlusion for recovery, occlusion is applied in cycles dispersed with periods of reperfusion. Generally, the protocol involves three or four cycles of five min occlusion above systolic blood pressure, followed by a five min reperfusion period, with a range of applied pressures previously reported (Thijssen et al. 2016) (Table 2.5). Further, the numbers of ischemic reperfusion cycles have varied in previous studies with further disparity between limb IPC and vascular occlusion for recovery. Studies have explored the impact of increasing the 'dose' of the traditional limb IPC protocol (i.e. 3-4 cycles) (Thijssen et al. 2016), specifically Cocking et al. 
(2017) examined cycling performance following five different limb IPC protocols. Responses to traditional limb IPC (4x5-min legs) were compared to: (i) $8 \times 5$-min legs and SHAM, (ii) 4x5-min unilateral legs, and (iii) 4x5-min arms. It was identified traditional limb IPC was associated with a $17 \mathrm{~s}$ faster cycling time compared to SHAM. However, applying increased ischemia ( $8 \times 5-\mathrm{min})$ had no impact on performance. Unilateral limb IPC was associated with $18 \mathrm{~s}$ slower performance compared to bilateral protocol and cycling times following remote and local limb IPC were not different. Therefore, it was concluded the traditional 4x5-min limb IPC stimulus resulted in the fastest cycling time compared to SHAM, there was no benefit of applying a greater number of cycles or employing unilateral limb IPC (Cocking et al. 2017). 


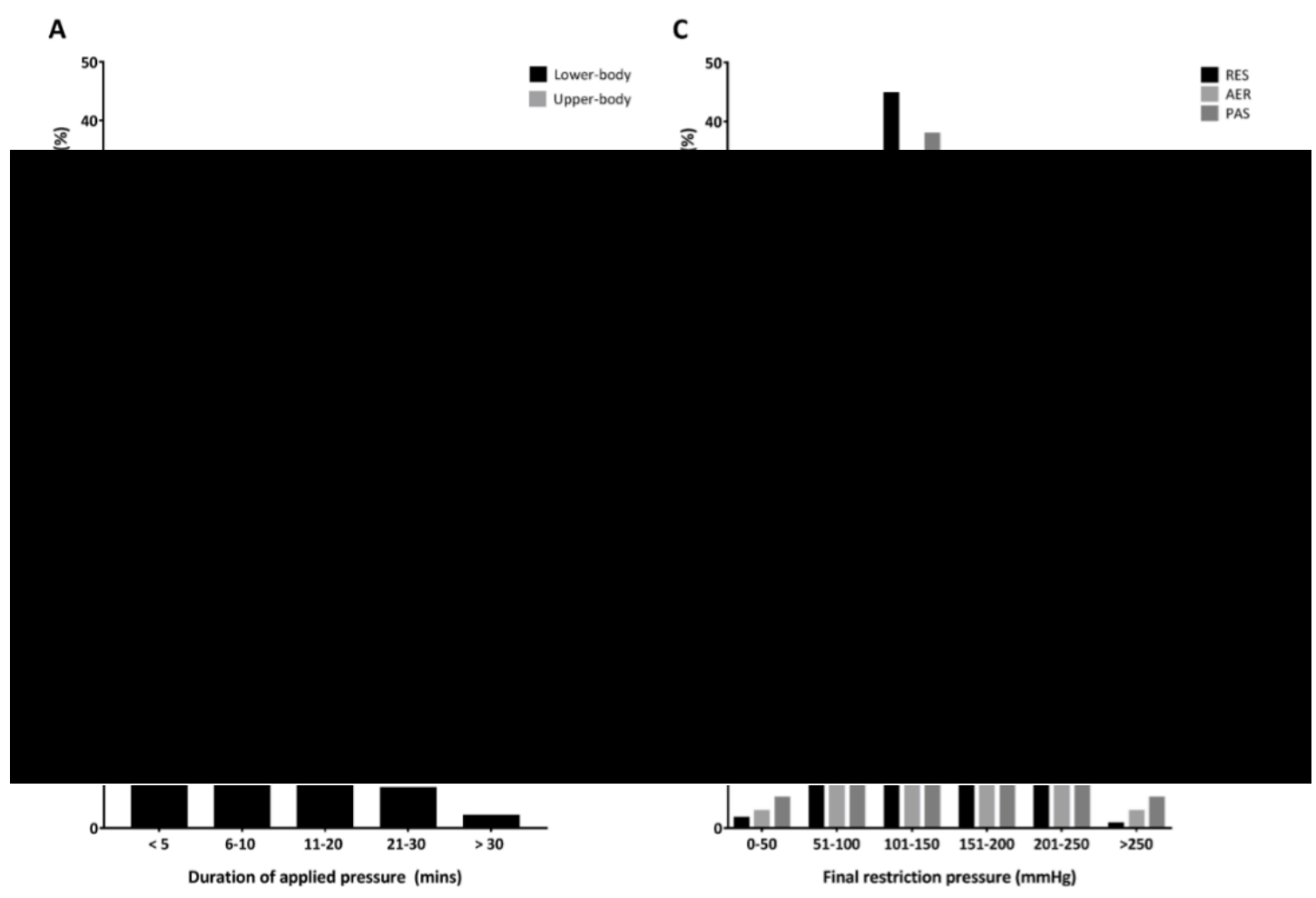

Figure 2.6 Prescription variables used in practice by performance scientists to implement limb ischemic preconditioning, identifying cuff width, restriction pressure and duration of applied pressure. (RES - resistance exercise; AER - aerobic exercise; PAS - passive) (From Patterson \& Brandner 2017) 


\subsubsection{Summary}

Individual differences (e.g. limb size) as well as methodological differences, for example restrictive cuff size and cuff pressure are variables that must be considered with limb IPC and vascular occlusion (Fahs et al. 2012). Manipulation of these variables will affect the degree to which blood flow is restricted and this may affect the acute responses (Fahs et al. 2012). Results from previous literature highlight the variation in physiological response and tolerability of vascular occlusion and limb IPC dependent on the cuff width and cuff inflation pressure used (Sharma et al. 2014). Currently, no optimal cuff width/pressure combination has been established for limb IPC or vascular occlusion; therefore, no standard exists regarding the application of this strategy. As such, potential issues arise in finding an optimal restriction pressure due to the numerous different cuff widths and pressures implemented (Jessee et al. 2016). Although precise recommendations are not available, it is in agreement that cuff pressure should be individualised to ensure it is high enough to occlude venous return from the muscle, but low enough to maintain arterial inflow into the muscle when applied during exercise and reports suggest pressure needs to be above systolic blood pressure for limb IPC. A balance between cuff width and cuff pressure needs to be established and determined within the literature to identify a suitable protocol to stipulate an optimal response to limb IPC and vascular occlusion and the effects it can have as a pre-competition and recovery strategy. Limb circumference and blood pressure should be considered to individualise occlusion pressure or determined as a percentage of AOP. Regarding dose of limb IPC and vascular occlusion, a consensus does not exist with regard to the protocol; the number of cycles, duration and the reperfusion periods vary considerably among studies, as well as period of cessation of the IPC procedure and onset of exercise. Despite this, this modality has vast applications to many different populations, both trained and untrained, as such, it is necessary to thoroughly understand the stimulus applied to prescribe the appropriate pressure to facilitate the desired response.

\subsection{METHODLOGICAL CONSIDERATIONS}

\subsubsection{Saliva as a diagnostic tool in sport and exercise}

Blood collection and muscle biopsies are considered gold standard for collection of biomarkers (Lindsay \& Costello 2016). However, as they are both invasive, saliva is becoming increasingly useful due to its relative ease, non-invasive and time efficient collection protocol (Lindsay \& Costello 2016). In comparison to serum and plasma, saliva collection requires less medical training and is safer to collect in the field than blood collection (Pritchard et al. 2017), alongside the benefits of rapid, frequent collection without stress to the participant (Chicharro et al. 1998; Cadore et al. 2008). The measurement of physiological biomarkers in whole saliva can provide a significant 
tool for assessing the immunological and endocrinological status associated with exercise and training (Papacosta \& Nassis 2011) and provides researchers with the ability to conduct repeated sampling over the course of minutes, hours, days, or longer (Dabbs \& La Rue 1991). Saliva originates mainly from 4 pairs of glands; parotid, sublingual, submandibular and minor (Proctor $\&$ Carpenter 2007) with relative contributions ranging from 4 to $65 \%$ (Chicharro et al. 1998). Each gland can produce variable amounts of salts, ions and proteins (Hu et al. 2004) which can be influenced by factors including psychological and hormonal status, physical exercise, flow rate, circadian rhythm, size and type of the stimulus (Chicharro et al. 1998; Schipper et al. 2007).

Saliva is primarily composed of water with an abundance of weak and strong ions, where the concentration is dependent on secretion stimulation type (Chiappin et al. 2007), however, saliva composition also consists of hormones, peptides, electrolytes, mucus, antibacterial compounds and various enzymes (Chicharro et al. 1998). The total concentrations of most of these compounds are much lower in saliva when compared with serum or plasma; however, they can provide a reliable reference for their respective blood concentrations (Cadore et al. 2008) as specific substances that circulate in the blood can enter into the salivary ducts by passive filtration through the membrane barrier (Bloomer 2015). Steroid hormones detectable in saliva include C, androgens including T, estrogens and progesterone (Papacosta \& Nassis 2011). Therefore, saliva has been reported to be an ideal medium for the analysis and diagnosis of exercise stress, due to containing a variety of enzymes, hormones, antibodies, antimicrobial constitutes and growth factors (Zelles et al. 1995; Rehak et al. 2000).

The use of salivary biomarkers allow prediction and evaluation (Lindsay \& Costello 2016), offering potential in both acute assessment of physiological and psychological stress and chronic assessment. Steroid hormones leak into saliva from plasma, however, only the free unbound fraction is involved. Albumin and sex-hormone binding globulin do not allow the bound fraction of the hormones to get into the saliva due to their molecular weights (Celec et al. 2009). Thus, salivary concentrations represent valuable markers for the free, unbound and bioactive fraction of the particular steroid (Arregger et al. 2007). Specifically for C, literature has suggested saliva to be a more robust measure of stress than serum $\mathrm{C}$ because salivary $\mathrm{C}$ is a better measure of adrenal function (Aardal \& Holm 1995). For instance, significant correlations have been reported between blood and salivary C concentrations at rest (Lippi et al. 2009; Rantonen et al. 2000). Post-exercise, significant correlations were found between salivary and blood concentrations of $\mathrm{C}$ following intense standardised exercise protocols (Cadore et al. 2008; Stupnicki \& Obminski 1992; O'connor \& Corrigan 1987), a $30 \mathrm{~s}$ wingate test (Crewther et al. 2010) and training matches (Moreira et al. 2009; Doan et al. 2007). Gozansky et al. (2005) provided evidence to support that salivary C represents the biologically active, free fraction of blood $\mathrm{C}$, in this study salivary $\mathrm{C}$ measures 
displayed a greater relative increase in response to exercise and intravenous administration of corticotrophin-releasing hormone compared with blood $\mathrm{C}$ measures. Therefore, reports suggested $\mathrm{C}$ in saliva may provide a better measure for the assessment of dynamic hypothalamic-pituitaryadrenal axis activity. Further evidence has been provided by Crewther et al. (2010) who validated the use of salivary $\mathrm{C}$ measures in response to a short, high-intensity cycle bout, also displaying that the hormonal changes to exercise are greater in saliva than corresponding blood measurements; significant $(\mathrm{p}<0.001)$ strong correlations were demonstrated between salivary $\mathrm{C}$ and plasma $\mathrm{C}$ between individuals $(\mathrm{r}=0.81-0.84)$ and within individuals $(\mathrm{r}=0.83-0.84)$.

Salivary hormone levels also reflect the free plasma concentration and biologically active fraction of $\mathrm{T}$ that is available to interact with androgen receptors (Kraemer \& Ratamess 2005). Several studies suggested that a relationship exists between measures of salivary $\mathrm{T}$ and strength-related performance capacity (Crewther et al. 2010; Beaven et al. 2008; Beaven et al. 2008; Crewther et al. 2009). Monitoring salivary $\mathrm{T}$ levels in sports is a practical way to determine the preferred volume and intensity of exercise and this in turn should lead to enhanced functional gains (Beaven et al. 2008). In males, significant correlations have been reported between salivary and blood concentrations of T at rest. (Vittek et al. 1981; Shirtcliff et al. 2002). Salivary measures of T have provided a reliable indicator of serum or plasma concentrations, as salivary $\mathrm{T}$ concentrations were found to be strongly correlated with measures of bioavailable T (Morley et al. 2006). In regard to acute exercise, Crewther et al. (2010) showed that the relative peak increase in salivary $\mathrm{T}$ to a short high- intensity sprint exceeded the relative increases in total and free $\mathrm{T}$ in plasma (between individuals $\mathrm{T}: \mathrm{r}=0.57-0.61 ; \mathrm{C}: \mathrm{r}=0.83-0.84$; within individuals $\mathrm{T}: \mathrm{r}=0.71-0.73$ ), suggesting that salivary measures are more sensitive for assessing the hormonal response to exercise.

Unfortunately, not all studies use the same assay procedures to measure salivary $\mathrm{T}$ and this may be problematic when attempting to make comparisons across studies. Likewise, differences in sample collection procedures, storage, and processing of saliva can influence. For example, Arregger et al. (2007) noted that saliva measures of T were strongly correlated to blood measures in a sample of eugonadic $(\mathrm{r}=0.92)$ and hypogonadic $(\mathrm{r}=0.97)$ men. However, in this study, an adapted radioimmunoassay procedure was used for the saliva $\mathrm{T}$ assay, rather than an enzymelinked immunosorbent assay (ELISA) procedure. In comparison, Bloomer (2015) compared saliva and serum free $\mathrm{T}$ concentrations in 20 healthy men using ELISA procedures. Men provided both a saliva and blood sample on the same day in the morning hours following an overnight fast. Special care was taken in the collection, storage, and processing of samples. Following complete thawing and mixing of samples, both fluids were analysed in duplicate using commercially available ELISA kits, both prior to and following centrifugation. Saliva T values were $348.8 \pm$ 
$210.0 \mathrm{pg} \cdot \mathrm{mL}-1$ with centrifugation, whereas serum $\mathrm{T}$ values were $8.3 \pm 3.7 \mathrm{pg} \cdot \mathrm{mL}-1$ with centrifugation. It was concluded, if utilising ELISA procedures, saliva and serum $T$ values cannot be used interchangeably, at least when utilising the ELISA procedures employed in this study. The results demonstrated that saliva and serum samples result in dramatically different values of free $\mathrm{T}$ when analysed using two commercially available ELISA procedures, as such, when measuring free $\mathrm{T}$ performance scientists should identify one method and maintain that method throughout all testing. However, when assay method is consistent, reports have shown salivary $\mathrm{T}$ levels are modestly correlated with serum levels for males; positive correlations were reported for T levels between saliva and total serum $\mathrm{T}(\mathrm{r}=0.71)$ and free $\mathrm{T}(\mathrm{r}=0.67)$ (Shirtcliff et al. 2002).

\subsubsection{Summary}

Within previous research, saliva samples for measuring levels of endogenous steroid hormones, have claimed that salivary assays "do not meet the criteria for routine diagnostic tests and their introduction into laboratory repertoire cannot be justified at present" (Wood 2009, p. 193). Whereas others have argued that "saliva...has proven to be reliable and, in some cases, even superior to other bodily fluids" (Gröschl 2008, p. 1759) for measuring hormone levels (Liening et al. 2010). However, specifically within sport, salivary $\mathrm{T}$ and $\mathrm{C}$ have previously been validated in response to short high-intensity exercise and are sensitive to changes caused by exercise (Crewther et al. 2010). The saliva measures more accurately reflect $(\mathrm{r}=0.92-0.97)$ blood free $\mathrm{T}$ or $\mathrm{C}$ concentrations to provide valuable data on the biologically active portion (1-5\% of total hormones) that is available to tissue (Crewther et al. 2010). Therefore, these findings support the use of saliva as a medium for steroid determination in sport.

\subsubsection{Safety Considerations}

There has been extensive evidence that indicated vascular occlusion combined with training can provide a unique, beneficial mode of exercise, even in clinical settings, because it produces positive training adaptations equivalent to the physical activity of daily life (10-30\% of maximal work capacity) (Abe et al. 2006). There are several uncertainties regarding the interplay of various extrinsic and intrinsic factors on its safety and efficiency (Kacin et al. 2015). Among extrinsic factors tourniquet cuff pressure, size and shape have been identified as key for safety and efficiency. Among intrinsic factors, limb anthropometrics, patient history and presence of cardiac, vascular, metabolic or peripheral neurologic conditions have been recognised as most important (Kacin et al. 2015). A review by Kacin et al. (2015) identified the following variables as the most probable health-hazards: (i) mechanical injury to the skin, muscle, and peripheral nerves, (ii) venous thrombosis due to vascular damage and disturbed hemodynamics and (iii) augmented arterial blood pressure responses due to combined high body exertion and increased peripheral vascular resistance. 
There are several safety considerations when using vascular occlusion in training when compared to regular exercise including the impact on the cardiovascular system (central and peripheral), muscle damage, oxidative stress and nerve conduction velocity (Loenneke et al. 2011). Caution should be heeded as excess pressures could induce substantial compression of not only vasculature but also neural tissue, especially if the cuffs are inflated during muscle contraction (Clevidence et al. 2012) causing symptoms such as numbness. Furthermore, research has reported that inappropriate implementation of vascular occlusion can result in detrimental effects, including petechial haemorrhage and dizziness (Scott et al. 2014).

A survey on the safety of occlusion training was published by Nakajima et al. (2006) based on reports from 105 training centres in Japan. A total of over 12600 individuals had trained using tourniquets in combination with different modes of exercise (walking, cycling, weight training). The most common side effects were subcutaneous hemorrhage (incidence 13.1\%) and temporary numbness (1.3\%). More serious side effects were rare; venous thrombosis $(0.055 \%)$, deterioration of ischemic heart disease $(0.016 \%)$, cerebral infarction $(0.008 \%)$, rhabdomyolysis $(0.008 \%)$ and pulmonary embolism $(0.008 \%)$. Although the estimated risk of venous thrombus is very low, it is a real risk, consequently, consideration must be given to personal or family history of conditions affecting blood flow through local vessels or the wider cardiovascular system. Furthermore, lack of blood perfusion to a limb and extreme physical exertion are both well-known causes of rhabdomyolysis. This is a clinical syndrome resulting from skeletal muscle damage and the release of potentially toxic substances into the circulation (Allison \& Bedsole 2003). It may be caused by trauma or muscle hypoxia and manifests as muscle pain and weakness. Therefore, consideration must be given in the case when other conditions associated with rhabdomyolysis are present (Kacin et al. 2015). Caution must be taken in individuals who lack any previous training history as unaccustomed exercise can also be associated with an increased risk of rhabdomyolysis. Participants with a history of or an increased risk of cardiovascular disease should be thoroughly screened prior to implementation of vascular occlusion (Kacin et al. 2015).

Vascular occlusion combined with training has also been associated with central responses of the cardiovascular system. Fahs et al. (2012) highlight that during vascular occlusion with exercise, awareness should be present that reductions in arterial blood flow to skeletal muscle increase the chemoreflex causing greater elevations in heart rate and blood pressure during exercise. For example, during walking exercise, occlusion has been shown to increase the heart rate and blood pressure response (Renzi et al. 2011). Furthermore, higher $(200 \mathrm{mmHg})$ cuff pressures increased heart rate and blood pressure to a greater extent during exercise compared to lower $(160 \mathrm{mmHg})$ cuff pressures (Sakamaki et al. 2008). Complete arterial occlusion (300mmHg) during unilateral 
elbow flexion resistance exercise has been shown to increase heart rate to a greater extent than moderate arterial occlusion (160 mmHg) (Fahs et al. 2012). In support, low intensity vascular occlusion training (20\% 1RM) has been found to result in slightly higher values for heart rate (109 vs $96 \mathrm{bpm}$ ) and systolic (182 vs $155 \mathrm{mmHg})$, diastolic (105 vs $99 \mathrm{mmHg}$ ) and mean arterial blood pressure (127 vs $113 \mathrm{mmHg}$ ) (Takano et al. 2005); however these values are well below changes that occur during high intensity resistance training (Loenneke et al. 2011). Nakajima et al. (2006) found that numbness was sometimes reported in response to a vascular occlusion exercise bout. This raises an important safety question, with respect to the numbness and possible nerve conduction blockage which is often seen following surgery or when external compressions are applied to a limb causing both ischemia and a slowing of nerve conduction velocity (Mittal et al. 2008). Clark et al. (2011) reported that nerve conduction velocity was unchanged after 4 weeks of low intensity vascular occlusion training (30\% 1RM). This was an expected outcome because the overall length of the restrictive exercise bout only lasted approximately $10-15 \mathrm{~min}$.

The potential concern with increasing pressures too high is that arterial blood flow maybe completely removed during the rest periods between sets and possibly during the exercise itself (Loenneke et al. 2014). Complete arterial occlusion is a safety concern because this can cause the formation of a thrombus and can induce a microvascular occlusion even after reperfusion (Laurentino et al. 2008). This post-reperfusion occlusion could result in muscle damage and/or cell necrosis (Laurentino et al. 2012; Loenneke et al. 2011). However, Madarame et al. (2010) reported that there was no increase in prothrombin fragments $1+2$ or thrombin-antithrombin III complex (markers of thrombin generation) following continuous occlusion at 30\% 1RM with 30 sec rest.

While low-load resistance exercise with occlusion can increase the strength and hypertrophy of skeletal muscle, concomitant increases in the strength of connective tissues may not occur due to decreased mechanical loading (Scott et al. 2014). A disproportionate increase in muscle and connective tissue strength may result in musculotendinous injury, particularly if heavy loads are subsequently used which can be lifted by the muscles but not tolerated by connective tissues (Kubo et al. 2006).

Despite the potential risks reported above, research to data has concluded that occlusion exercise is a safe method for training athletes, healthy persons (Clark et al. 2011) and potentially those individuals with varying medical concerns (Nakajima et al. 2006). Supporting evidence by Loenneke et al. (2012), reports that vascular occlusion appears to be safe and poses no greater risk than traditional high intensity resistance exercise with respect to the cardiovascular system, muscle damage, oxidative stress and nerve conduction velocity. There have been no long term negative 
consequences of occlusion reported within the literature; it is important to acknowledge the method and protocol has been established and derived from a clinical environment where it is used to reduce myocardial infarct size and therefore has received a large body of research (Mattocks et al. 2017). For clinical application, tourniquet pressure above $170 \mathrm{mmHg}$ for upper and $270 \mathrm{mmHg}$ for lower limbs is usually used (Fitzgibbons et al. 2012). However, within an athletic setting, the use of arbitrary pressures may place some individuals under complete arterial occlusion which may increase the potential risk of an adverse event and therefore highlights the importance, not only for efficacy of the intervention, but also safety, to individualise the occlusion protocol (Dankel et al. 2017). Furthermore, applying higher relative pressures results in the greatest cardiovascular response, higher perceptual ratings, and greater decrease in exercise volume compared to lower restriction pressures (Mattocks et al. 2017). Therefore, the perceptual responses from lower relative pressures may be more appealing and provide a safer and more tolerable stimulus for individuals.

\subsubsection{Summary}

Taken from Nakajima et al. (2006), it would be recommended to screen participants prior to completion of vascular occlusion for several contraindications, for instance, history of deep-vein thrombosis, pregnancy, varicose veins, and several other factors relating to the patient's history of disease and inactivity. This approach may be beneficial for identifying those at risk of detrimental complications during vascular occlusion. Nonetheless, when used in a controlled environment by trained and experienced personnel, vascular occlusion training appears to provide a safe training alternative for most individuals regardless of age and training status (Loenneke et al. 2011), with previous in depth reviews suggesting correct implementation of vascular occlusion presents no greater risk than traditional and heavy exercise modes ( Loenneke et al. 2011).

\subsection{CONCLUSIONS AND RECOMMENDATIONS}

Pre-competition and recovery are two areas that can improve athletic performance, in which performance scientists can implement strategies on the day prior to competition to optimise performance, for example through the attenuation of $\mathrm{T}$ by completing morning exercise prior to afternoon performance. There is also opportunity to assist in the rate of recovery from exercise induced fatigue and muscle damage. Previous strategies, although beneficial, maybe impacted by competition schedules, timings and equipment, therefore, vascular occlusion and limb IPC offer a strategy that may have the ability to be applied both for pre-competition performance improvement and increase rate of recovery. 
Vascular occlusion and limb IPC were initially utilised within a clinical setting, derived from a medical technique to reduce myocardial infarct size, specifically through the release of adenosine which increases $\mathrm{O}_{2}$ delivery by dilation of blood vessels. Expression of signalling molecules leads to activation of mediators that transmit a protective signal in the cytosol (Thijssen et al. 2016), ultimately converging at the mitochondria and crucially opening the mitochondrial permeability transition pore (Davidson et al. 2006). The clinical literature highlights an early and late phase mediated by adenosine via different end-organ receptors (Fryer et al. 2002), in which the early phase provides protection 3-4 $\mathrm{h}$ after the initial ischemic stimulus, whereas the late phase appears $24 \mathrm{~h}$ and lasts up to $72 \mathrm{~h}$ following brief periods of IPC (Baxter et al. 1997).

This concept, based on assumption that a tissue with some history of ischemic conditions eventually becomes resistant to ischemia and its potential positive effects (Ovize et al. 2013) attracted researchers in sports medicine and exercise science, now applied in three distinct areas of performance; pre-competition, training and recovery.

Within this review, the focus was on pre-competition and recovery strategies; the evidence supports the use of vascular occlusion as a pre-competition strategy, the studies examined showed a benefit to performance, when combining vascular occlusion with resistance exercise. Although traditionally used for training purposes, research has identified the acute response of completing resistance exercise with vascular occlusion and sequential effect on physiological mechanisms including recruitment of fast twitch motor units and hormonal response, however application precompetition is yet to be assessed.

When applied during supine rest, limb IPC has reported to improve exercise time to exhaustion, repeated sprints and single anaerobic performance, with consistent support for its application to improve swimming performance. The limb IPC strategy employed varies within the research, with differences in cycles of occlusion between sport modalities (3-4 cycles) and also the timeline of cessation of limb IPC to the onset of performance, varying from five min prior to endurance performance to 30-90 min prior to anaerobic performance. There is limited support on the use prior to submaximal exercise and there is disparity in findings of implementing limb IPC prior to repeated sprint performance, therefore further research is required on the use of limb IPC, potentially the standardisation of a protocol regarding cuff pressure, width and timings may resolve some of the disparity within the literature to date. Further research is also required on protocols and testing specific to sports competitions and ensuring protocols are individualised.

Some positive results were identified using vascular occlusion as a recovery strategy, proposed to be due to increased blood flow associated with adenosine levels (Liu et al. 1994) and improved 
muscular $\mathrm{O}_{2}$ utilisation following reperfusion, leading to a more rapid return of muscle function (Beaven et al. 2012). However, this is speculated, in addition to current inconsistent results, prevents provision of a definitive conclusion and application in an athletic environment. Further research is required to identify the physiological mechanisms involved in vascular occlusion for recovery.

Across both pre-competition (with and without resistance exercise) and recovery, large methodological differences exist, for example, restrictive cuff size and cuff pressures are variables that must be considered with limb IPC and vascular occlusion. Manipulation of these variables will affect the degree to which blood flow is restricted, consequently affecting the acute response (Fahs et al. 2012). No optimal cuff width/pressure combination has been established which is problematic as no standard exists regarding the application of this strategy when combined with resistance exercise, applied at passive rest for limb IPC pre-competition or use as a recovery strategy.

To conclude, further research is required in athletes to determine the effects of vascular occlusion and limb IPC, taking into account emerging research on the effect of occlusion protocols (e.g. cuff pressure, time between occlusion and performance) and continued research into the underlying physiological mechanisms to determine how this strategy can be best applied to improve athletic performance. Identified areas of research, as a consequence of this review, are specified below:

- Future trials are required to identify the use of vascular occlusion with high intensity resistance exercise to be used a pre-competition strategy (Chapter 4).

- Limited research (one study) has investigated the effect of early and late phase of limb IPC on athletic performance and therefore requires further attention both regarding physiological mechanisms and impact on performance (Chapter 5).

- The use of vascular occlusion as a recovery strategy is unclear, data at present is not conclusive and the physiological mechanisms are unknown (Chapter 6).

- The effect of limb IPC on repeated sprint is inconclusive and more information is required before applying in sport

- Optimal cuff pressure, length of occlusion and rest time between vascular occlusion/limb IPC and onset of performance requires further investigation for both pre-competition and recovery

- Performance tests need to be specific to elite competition performance and tested with athletes as currently practice is inferred from a healthy, un-trained population (Chapter 4$6)$. 


\section{Chapter 3 - General methods}

\subsection{INTRODUCTION}

This chapter provides details of methods that were used in two or more of the subsequent experimental chapters. Methods that were unique to a particular study are presented in the methods section of the respective study chapter.

\subsection{PARTICIPANTS}

Swansea University ethics committee approved all of the studies in this thesis (Appendix A). All participants were informed of the experimental procedures and the purpose of the study (Appendix B), along with possible risks associated with the study and provided written informed consent before participation (Appendix C). All participants were free to withdraw at any time during the studies. For all studies, elite, well-trained athletes were recruited to participate. All participants had a minimum of five years training experience within their specific sport and were competitive at National standard or above. The participant characteristics for each study can be seen in further detail in Table 3.1 .

Estimated day of menstrual cycle was calculated from the information that female participants provided at the first experimental trial. Consistent with the method reported by Liening et al. (2010), the day of menstrual cycle at the subsequent experimental trials was calculated by adding seven days to the day of menstrual cycle after the first-time point, and repeated for subsequent sessions. The self-reported average cycle length was used to account for those participants who had begun a new cycle between the collection dates.

\subsection{BLOOD PRESSURE ASSESSMENT}

Blood pressure assessment was completed in all experimental chapters. Participants were required to sit in a chair with a back support, with both feet flat on the floor for ten min prior to obtaining measurement (Pickering et al. 2005; Daskalopoulou et al. 2015). Once this position was established, the cuff size was checked ensuring the bladder covered at least $2 / 3$ of the distance around the arm and was not overlapping. The cuff was placed on the bare arm, with the midline of the inflatable bladder positioned over the brachial artery at the mid-point of the upper arm, approximately two fingerbreadths above the arm crease (Frese et al. 2011). Due to the effects of hydrostatic pressure, the position of the arm when blood pressure is measured can affect the 
reading (Daskalopoulou et al. 2015). Therefore, the arm was supported so that the cuff was at the level of the heart. An automated blood pressure monitor (Omron Healthcare, Europe) was used to determine systolic and diastolic blood pressure. Pickering et al. (2005) recommended the average of two readings taken at an interval of at least one min. If the difference between the first and second reading was $>5 \mathrm{mmHg}$, an additional reading was taken and the average of the three recorded.

\subsection{OCCLUSION CUFFS AND PRESSURE}

Occlusion cuffs were applied to the most proximal point of the thighs in all studies (Figure 3.1). The cuffs (11 cm; Sports Rehab Tourniquet, Sportsrehab) contained a pneumatic bag along its inner surface that was connected to a pressure gauge and manually inflated to either $15 \mathrm{mmHg}$ in control conditions or an individualised cuff pressure, consistent across all experimental chapters. The individualised cuff protocol was selected as a stimulus likely to cause lower limb ischemia, but acknowledging that the same pressure may not restrict the same amount of blood flow in every individual since the amount of tissue surrounding the blood vessel may influence the pressure exerted on the vasculature and therefore the degree of blood flow restriction (Hargens et al. 1987). Initial investigations by Loenneke et al. (2012) suggested pressures to restrict blood flow of the lower body should be determined by limb circumference. A more recent study (Loenneke et al. 2015) replicated these previous findings that thigh circumference is the biggest predictor of arterial occlusion in the lower body $(\beta=0.570)$. This reinforces pressures in the lower body should be to some degree individualised according to thigh size, meaning bigger thighs required greater pressures, consistent for both sexes. However, this research also identified brachial systolic blood pressure may be an additional significant predictor $(\beta=0.231)$. Therefore, cuff pressure was calculated using Equation 3.1 from the research complete by Loenneke et al. (2015) using thigh girth and blood pressure measurements as detailed in 3.3 and 3.5. This accounted for the reports suggesting pressures needed to be above systolic blood pressure for limb IPC (Horiuchi 2017; Pope et al. 2013). Once cuff pressure was determined, $60 \%$ of arterial occlusion was calculated following previous research identifying no additional benefit of increasing pressure higher (e.g. Counts et al. 2016) (for further information see section 2.4.1).

Equation 3.1:

Arterial occlusion $(\mathrm{mmHg})=5.893(\mathrm{~cm}-$ Thigh circumference $)+0.734(\mathrm{mmHg}-\mathrm{DBP})+0.912$ $(\mathrm{mmHg}-\mathrm{SBP})-220.046$

Where DBP is diastolic blood pressure and SBP is systolic blood pressure 
Table 3.1: Summary of participant characteristics for each study

\begin{tabular}{|c|c|c|c|c|c|c|}
\hline & Sport & $\mathbf{N}$ & Gender & Age (yrs) & Height (cm) & $\begin{array}{l}\text { Weight } \\
\text { (kg) }\end{array}$ \\
\hline $\begin{array}{l}\text { Study } 1 \\
\text { (Chapter 4) }\end{array}$ & $\begin{array}{l}\text { Weightlifting } \\
\& \text { athletics }\end{array}$ & 22 & $\begin{array}{l}10 \text { males } \\
12 \text { females }\end{array}$ & $\begin{array}{l}21 \pm 3 \\
26 \pm 5\end{array}$ & $\begin{array}{l}188 \pm 9 \\
162 \pm 8\end{array}$ & $\begin{array}{l}85 \pm 10 \\
64 \pm 9\end{array}$ \\
\hline $\begin{array}{l}\text { Study } 2 \\
\text { (Chapter 5) }\end{array}$ & Rugby union & 24 & All males & $21 \pm 3$ & $185 \pm 9$ & $97 \pm 10$ \\
\hline $\begin{array}{l}\text { Study } 3 \\
\text { (Chapter 6) }\end{array}$ & Swimming & 20 & $\begin{array}{l}14 \text { males } \\
6 \text { females }\end{array}$ & $\begin{array}{l}21 \pm 2 \\
19 \pm 1\end{array}$ & $\begin{array}{l}182 \pm 3 \\
166 \pm 6\end{array}$ & $\begin{array}{l}77 \pm 5 \\
59 \pm 7\end{array}$ \\
\hline
\end{tabular}




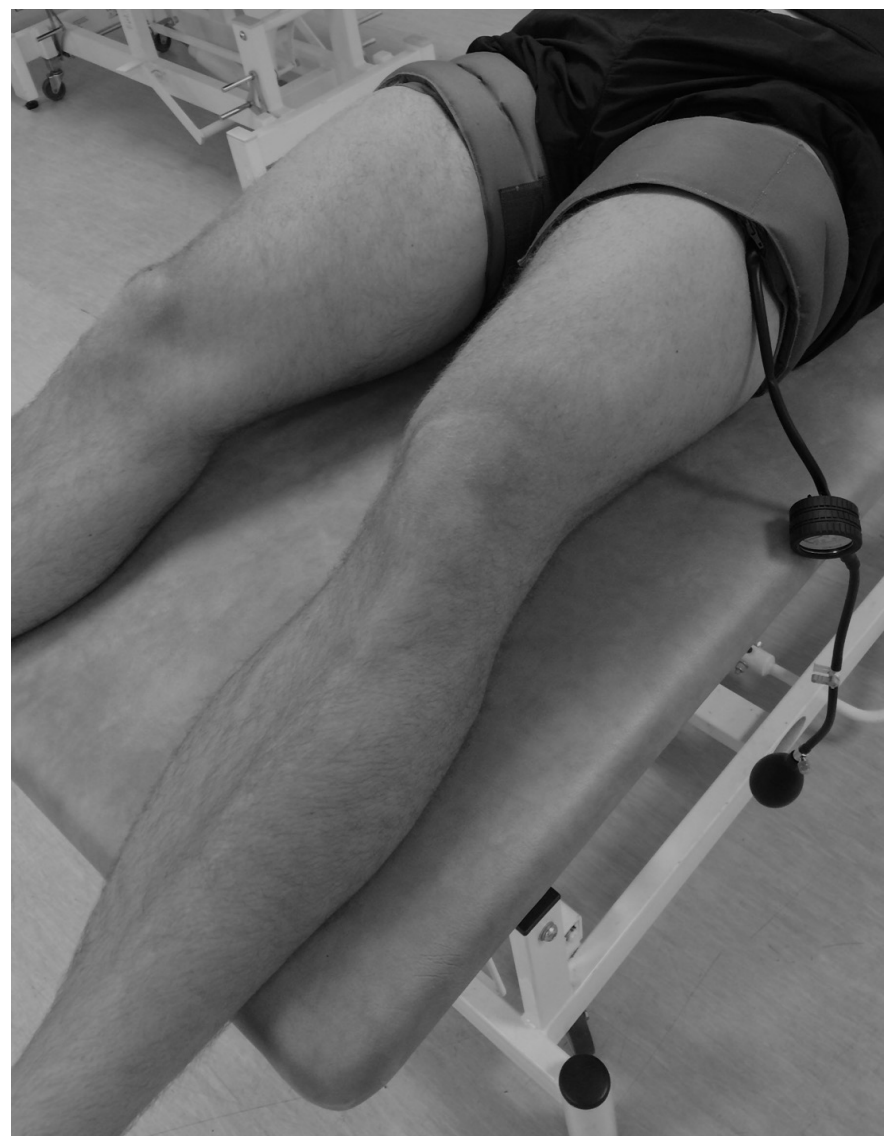

Figure 3.1 Application of occlusion cuffs whilst in a supine position 
The control condition was determined following previous research in which 10-20 $\mathrm{mmHg}$ has been previously used within a control condition (Jean-St-Michel et al. 2011; Bailey et al. 2012; Patterson et al. 2015; Tocco et al. 2015) and has been shown to cause no alteration to the arterial inflow but allows increased control over the placebo effect as cuffs are worn in both conditions.

\subsection{THIGH GIRTH ASSESSMENT}

Thigh girth measurements were required to estimate cuff inflation pressure. The same individual completed the thigh girth measurements $(\mathrm{CV}<0.54 \%$ within-individual assessment $)$; measurements followed the International Society for the Advancement of Kinathropometry protocol. Circumference of the right thigh was measured at $33 \%$ of the distance from the inguinal crease and top of the patella on the midline of the thigh. Circumferences were measured at this mark to try and capture an accurate representation of the site at which the cuffs would be applied, in accordance with Loenneke et al. (2015). Thigh girth was measured, rested and then re-tested; measurements were required to be within $5 \%$ otherwise a third measurement was to be completed. The average was recorded and used to determine cuff pressure.

\subsection{BLOOD COLLECTION}

Capillary whole blood samples were collected for all experimental chapters; to complete this a small incision was performed on the fingertip using a single-use disposable lancet (Accu-check, Roche Diagnostics, $\mathrm{GmbH})$. After applying gentle pressure to the finger, a blood sample was collected in a capillary tube, the volume was specific to the measurement device being used as detailed below.

\subsubsection{Blood Lactate}

For chapters 4 and 6, a $20 \mu$ capillary tube was used to collect a blood sample as detailed above (Davison et al. 2000), this was immediately placed in a pre-filled reaction tube, mixed with a lysing stabilising agent in a safe-lock vial (Davison et al. 2000). The sealed vial was shaken and analysed. The blood sample was analysed for lactate using an automated lactate analyser (Biosen C-Line Clinic, EKF Diagnostic GmgH, Barleben, Germany). Prior to each testing session the analyser was calibrated with a standard known solution $\left(12 \mathrm{mmol} \cdot \mathrm{l}^{-1}\right)$ before any analysis was carried out (Davison et al. 2000). Previous research has identified the Biosen as a valid and reliable analyser for a full range of blood lactate values collected from a series of maximal and submaximal exercise tests, at a range of intensities from rest $\left(0.85 \mathrm{mmol} \cdot \mathrm{L}^{-1}\right)$ to volitional exhaustion $\left(16.37 \mathrm{mmol} \cdot \mathrm{L}^{-1}\right)$ (Davison et al. 2000). The Biosen was compared against Yellow Springs Instrument (YSI) across a range of samples taken from rest to after maximal exercise; test re-test of the Biosen for reliability 
and stability of samples were assessed. When compared against the YSI, a very strong relationship was evident with the Biosen (coefficient of determination 0.975), the average bias was 0.37 $\mathrm{mmol} \cdot \mathrm{L}^{-1}$ and $95 \%$ limits of agreement were 1.59 to $-0.85 \mathrm{mmol} \cdot \mathrm{L}^{-1}$, with the bias consistent across a range of values. For test re-test reliability, a high coefficient of determination was established $\left(r^{2}=0.995\right)$, however paired t-test showed the second measurement was significantly higher $(6.44$ $\left.\pm 3.62 \mathrm{mmol} \cdot \mathrm{L}^{-1}\right)$ than the first $\left(6.41 \pm 3.61 \mathrm{mmol} \cdot \mathrm{L}^{-1}\right)$, although the magnitude of increase was very small $\left(0.03 \mathrm{mmol} \cdot \mathrm{L}^{-1}, 95 \%\right.$ CI $\left.0.01-0.05\right)$. The coefficient of variation of test re-test data for the Biosen was $1.4 \%$ compared to $1.2-3.7 \%$ for YSI. The benefit of the Biosen was also displayed when assessing stability, there were no differences between samples when measured immediately or after $7 \mathrm{~h}$ or $24 \mathrm{~h}$ when kept at room temperature $\left(18-22^{\circ} \mathrm{C}\right)$.

For chapter 5, blood lactate was assessed using an ISTAT 1 analyser (Abbott, point of care testing) as this was being used to determine additional blood gases and $\mathrm{pH}$ as well. Further details of this analyser are provided in section 3.6.2.

\subsubsection{Blood $\mathrm{pH}$ and gases}

To measure $\mathrm{pH}$ and blood gases (blood lactate, $\mathrm{pH}$, percentage oxygen saturation $\left[\mathrm{sO}_{2} \%\right.$ ], partial pressure of oxygen $\left[\mathrm{PO}_{2}\right]$, partial pressure of carbon dioxide $\left[\mathrm{PCO}_{2}\right]$, total carbon dioxide $\left[\mathrm{TCO}_{2}\right]$, bicarbonate $\left[\mathrm{HCO}_{3}\right]$ and Base Excess $\left.[\mathrm{BEecf}]\right)$ in chapter 5, a $60 \mu \mathrm{l}$ sample was taken from the finger as described above and was analysed using an ISTAT 1 analyser (Abbott, point of care testing) and CG4+ cartridges (Abbott, point of care testing). Prior to data collection the ISTAT was calibrated according to the manufacturer's specifications by an electronic simulator and level one and three ISTAT control solutions (Abbott, point of care testing). Cartridges were stored prior to use as per manufacturer's instructions $\left(2-8^{\circ} \mathrm{C}\right)$ and removed to room temperature $\sim 5$ min prior to use. Upon completion of collection, the capillary blood sample was immediately expelled from the capillary tube into the sample well of the cartridge to prevent exposure to $\mathrm{PO}_{2}$ and $\mathrm{CO}_{2}$, this was analysed immediately. To allow for immediate analysis two ISTAT 1 analysers were used, however the same analyser was used for all data points of one individual to avoid inter-sample variation.

In each disposable cartridge, the measurement is based on ion-selective electrodes with potentiometric measurement for $\mathrm{pH}$ and $\mathrm{PCO}_{2}$ (analytical range $\mathrm{pH} ; 6.5-8.0, \mathrm{PCO}_{2} ; 0.67-17 \mathrm{kpa}$ ) and on a Clark electrode and amperometric measurement for $\mathrm{PO}_{2}$ (analytical range $\mathrm{PO}_{2} ; 0.67-107$ kpa), then comparison with an internal calibrator (Verwaerde et al. 2002). Oxygen saturation is calculated using an algorithm involving $\mathrm{pH}, \mathrm{PO}_{2}$ and $\mathrm{PCO}_{2}$; further measurements are calculated using the according equations (Verwaerde et al. 2002): 
Equations:

(3.2) $\log \mathrm{HCO}_{3}{ }^{-}=\mathrm{pH}+\log \mathrm{PCO}_{2}-7.608$

(3.3) $\mathrm{TCO}_{2}=\mathrm{HCO}_{3}{ }^{-}+0.03 \mathrm{PCO}_{2}$

(3.4.) $\mathrm{BEecf}=\mathrm{HCO}_{3}{ }^{-}-24.8+16.2(\mathrm{pH} 7.4)$

Blood gases and $\mathrm{pH}$ analysed with ISTAT have previously been compared against two autocalibrated bench top analysers (Radiometer analysers ABL 700 and 520) (Verwaerde et al. 2002). The ISTAT correlated well with the two control analysers $(r>0.993)$, with differences of 0.010 for $\mathrm{pH}, 0.19 \mathrm{kpa} \mathrm{PCO}_{2}$ and $0.41 \mathrm{kpa}$ for $\mathrm{PO}_{2}$ showing accuracy in comparison to alternative analysers (Verwaerde et al. 2002). This is supported by Kok et al. (2015) comparing the ISTAT CG4+ to clinical laboratory analysers (e.g. XE-5000, ABL800 FLEX). There was a good correlation (defined as a bias of $<10 \%$ between the ISTAT and the reference method) for $\mathrm{pH}, \mathrm{PCO}_{2}, \mathrm{HCO}_{3}$ and $\mathrm{sO}_{2} \%$. Dascombe et al. (2007) also confirmed intra-test reliability of the ISTAT analyser; ICC for all analytes were observed to be strong following maximal intensity exercise (ICC $=0.77-0.95$; where 0.7-0.7 deemed strong correlation) and technical error of measurement (TEM) of $<15 \%$ was deemed acceptable ( $\mathrm{pH} ; 0.24 \%$, Blood lactate; $3.12 \%$, all other measured blood gas parameters 2.02-8.85\%) confirming reliable analysis when using the ISTAT portable analyser.

\subsubsection{Creatine Kinase}

EIMD has frequently been examined using CK activity as an indirect marker in sport (Peake et al. 2005; Russell et al. 2016). Creatine kinase is an enzyme that catalyses the transfer of phosphate from PCr to ADP (Koch et al. 2014). The associated morphological changes of skeletal muscle damage within the muscle cell is accompanied by the leakage of proteins out of the cell and into the blood circulation (Hortobagyi \& Denahan 1989), therefore increases in CK in the blood stream are considered to be the result of increased membrane permeability due to damage. Despite suggested limitations associated with CK responses that are presented within literature (e.g. variance across individuals due to ethnicity, gender and age) (Baird et al. 2012), CK has been reported to be an indirect marker of EIMD (Koch et al. 2014).

For determination of $\mathrm{CK}$ in chapter 6 , a $120 \mu \mathrm{L}$ capillary blood sample was collected as descried above and immediately centrifuged (Labofuge 400R, Kendro Laboratories, Germany) at $3000 \mathrm{rpm}$ for $10 \mathrm{~min}$ for the extraction of plasma. This was subsequently stored at $-80^{\circ} \mathrm{C}$ until later analysis. The plasma samples were left to thaw before $6 \mu \mathrm{L}$ was used in the analysis of CK using an automated analyser (ABX Pentra 400, Horiba ABX, Montpellier, France), determined spectrophotometrically according to manufacturer procedures using a commercial kit. Prior to any analysis, calibration was completed using ABX Pentra multical and controls (ABX Pentra CK 
Control, $\mathrm{N}$ control, $\mathrm{P}$ control) and ensured this was within the defined range according to the manufacturer. Sample testing was carried out in duplicate, intra sample CV values were $<2.0 \%$. Coudene et al. (2005) have previously reported ABX Pentra $400 \mathrm{CV}$ values were within expected values when within-run imprecision (0.79\%) and between-run imprecision for serum and plasma assay (2.61\%). When compared with the Olympus AU640 a very strong correlation was reported ( $\mathrm{r}=0.98$ ). It was concluded the Pentra 400 had good reliability and practicality (Coudene et al. 2005).

\subsection{HORMONAL ANALYSIS}

Saliva collection, for chapter 4 and 6 , required passive drooling $(\sim 2 \mathrm{ml})$ into a sterile vial (SalivaBio, Salimetrics LLC, USA) following research suggesting this as the most reliable option (Granger et al. 1994) and steroid hormones not affected by flow rate (Papacosta \& Nassis 2011). Participants were required to refrain from brushing of teeth, drinking hot fluids, or eating hard foods in the $2 \mathrm{~h}$ before sampling to minimise the possibility of any blood contamination of saliva. This is a result of research showing concentrations of $\mathrm{T}$ increase in saliva following tooth brushing (Schramm et al. 1993) and research highlighting blood steroid hormone concentrations are generally an order of magnitude higher than that found in saliva (e.g. plasma cortisol is typically 200-800 $\mathrm{n} \cdot \mathrm{mol}^{-1}$ but in saliva is only 3-30 $\mathrm{n} \cdot \mathrm{mol}^{-1}$ ), therefore blood leakage into the mouth might lead to increased concentrations of steroids, which is in proportion to the leakage of blood into saliva (Schwartz \& Granger 2004). ELISA can detect concentrations of salivary steroids as a low as $2 \mathrm{pg} \cdot \mathrm{ml}^{-1}$ with sensitivity of $1 \mathrm{pg} \cdot \mathrm{ml}^{-1}$ (Lindsay \& Costello 2016). As a result, large concentration differences between blood and saliva exist, therefore robust saliva collection (as detailed above) is imperative for precise and accurate results as contamination of blood e.g. bleeding of gums from biting hard foods can significantly alter concentrations of specific analytes (Hofman 2001; Lindsay \& Costello 2016).

Furthermore, food or drink high in sugar content, caffeine or acidity can cause increased acidity, lowering mouth $\mathrm{pH}$ levels and leading to compromised antibody-antigen binding and enzyme activity thus leading to invalid immunoassay results (Granger et al. 2004).

All samples were chilled on ice immediately after collection, were stored at $-15^{\circ} \mathrm{C}$ within one $\mathrm{h}$ after collection to avoid bacterial growth and were transferred to $-80^{\circ} \mathrm{C}$ within $4-12 \mathrm{~h}$ of collection as previously recommended (Hansen et al. 2008). Post thawing, centrifugation (Micro Centaur, MSE, London, United Kingdom; five min at 3000 revolutions $\cdot \mathrm{min}^{-1}$ ) preceded duplicate analysis of $\mathrm{T}$ and $\mathrm{C}$ concentrations due to known reliable reflections of gonadal function (Vittek et al. 1987; Dabbs 1990) using indirect ELISA kits (Salimetrics Europe Ltd., Suffolk, U.K.) and optical 
density, determined on a plate reader at $450 \mathrm{~nm}$ in accordance to the manufacturer. The lowest detection limits for $\mathrm{T}$ and $\mathrm{C}$ were $6.1 \mathrm{pg} \cdot \mathrm{ml}^{-1}$ and $0.012 \mu \mathrm{g} \cdot \mathrm{dl}^{-1}$ respectively, and inter-assay $\mathrm{CV}$ values were $<10.0 \%$. To eliminate inter-assay variance, each athlete's samples were run within the same assay.

Saliva is becoming increasingly useful due to its relative ease, non-invasive and time efficient collection protocol (Lindsay \& Costello 2016) which is safer in the field than blood collection (Pritchard et al. 2017). Saliva has been reported to be an ideal medium for the analysis and diagnosis of exercise stress, $\mathrm{C}$ and $\mathrm{T}$ whose concentrations represent serum-free levels (Kumar et al. 2005) are reliable in comparison to serum for reflecting gonadal function and circadian patterns (Johnson et al. 1987; Dabbs 1990). Validation studies have demonstrated moderate to strong correlations $(\mathrm{r}=0.7-0.92)$ between salivary $\mathrm{T}$ and $\mathrm{C}$ concentrations and the corresponding blood total hormones at rest (Arregger et al. 2007; Vining et al. 1983). The saliva measures more accurately reflect $(\mathrm{r}=0.92-0.97)$ blood free $\mathrm{T}$ or $\mathrm{C}$ concentrations to provide valuable data on the biologically active portion (1-5\% of total hormones) that is available to tissue (Crewther et al. 2010). Although there is some disparity in the literature between saliva and plasma (between individuals $\mathrm{T}$ : $\mathrm{r}=0.57-0.61 ; \mathrm{C}: \mathrm{r}=0.83-0.84)$, significant strong correlations have been observed between salivary $\mathrm{T}$ and plasma $\mathrm{T}$ within individuals $(\mathrm{r}=0.71-0.73)$ and similarly for salivary $\mathrm{C}$ and plasma $\mathrm{C}$ within individuals $(\mathrm{r}=0.83-0.84)$ (Crewther et al. 2010). Therefore, salivary $\mathrm{T}$ and $\mathrm{C}$ has previously been validated in response to short high-intensity exercise, sensitive to changes caused by exercise (Crewther et al. 2010) (Further information refer to section 2.7.1).

\subsection{NEUROMUSCULAR PERFORMANCE}

Neuromuscular performance assessment was completed in chapters 4 and 6.

\subsubsection{Data collection}

The ground reaction force (GRF) was collected using a portable force platform with a built-in charge amplifier (Type 9286BA, Kistler Instruments Ltd., Farnborough, UK). In agreement with previous research (Owen et al. 2014), the vertical force range was set at $20 \mathrm{kn}$ and the GRF was sampled at $1000 \mathrm{~Hz}$ through a 16-bit analogue to digital converter (Kistler instruments Ltd., Farnborough, UK) using Kistler bioware software. The equipment was factory calibrated and its calibration was checked prior to each testing session using known masses. Both CMJ (chapter 4 and 6) and isometric mid-thigh pull (IMTP) (chapter 4) were performed. 


\subsubsection{Determination of body weight}

As identified in previous research (Owen et al. 2014), body weight was determined by taking the mean GRF value, as measured by the force platform, for one and half $\mathrm{s}$ of the stance phase immediately before the signal to jump being given.

\subsubsection{Countermovement jump}

All participants were familiar with CMJ testing and received verbal instructions prior to testing. To isolate the lower limbs, participants were instructed to stand with arms akimbo (Hatze 1998). Participants remained stationary for at least $2 \mathrm{~s}$ in an upright position for determination of body mass and as a reference from which jump initiation time was determined. After the initial stationary phase, participants performed a CMJ, dipping to a self-selected depth and then performing a vertical jump to achieve maximum height. Participants landed on the force platform and arms kept akimbo throughout the movement. Participants completed two maximum jumps with $90 \mathrm{~s}$ rest between efforts. Before completion, all participants performed a standardised warmup protocol ( $\sim 5 \mathrm{~min})$ consisting of activation exercises and dynamic stretches (Appendix D).

The vertical component of the GRF and the participant's body mass was used to determine instantaneous velocity and displacement of the centre of gravity. Jump height (JH) was defined as the difference in vertical displacement of the centre of gravity between take-off (toes leave the force plate) and maximum displacement (West et al. 2011). Instantaneous power output was determined using Equation 3.5 and the highest value produced was deemed PPO in accordance with previous methods (Owen et al. 2014; West et al. 2011). Peak velocity $\left(\mathrm{V}_{\max }\right)$ was taken as the highest rate of force development (RFD) during the concentric or eccentric phase of the jump. The concentric phase was defined as succeeding the point that the instantaneous velocity of the centre of gravity equaled zero after the initiation of the jump (West et al. 2011).

Equation 3.5

Power $(\mathrm{W})=$ vertical GRF $(\mathrm{N}) \mathrm{x}$ Vertical velocity of center of gravity $\left(\mathrm{m} \cdot \mathrm{s}^{-1}\right)$

\subsubsection{Isometric mid-thigh pull}

For IMTP testing, participants stood on a portable force platform (Kistler Instruments Ltd., Farnborough, United Kingdom), which was positioned in the base of a portable power rack, directly under the bar. Participants assumed a body position like the second pull of a power clean with a flat trunk position and their shoulders in line with the bar; maintaining knee angles of $\sim 120$ $130^{\circ}$ (checked with goniometry, Smith \& Nephew, Hull, United Kingdom) (West et al. 2011). Once the bar height was established, to allow for differences in participant statures, the participants stood on the force platform and were strapped to the bar (West et al. 2011). A quiet standing phase of $5 \mathrm{~s}$ was completed before initiation of the IMTP. Participants were instructed to pull as hard 
and as fast as possible on the bar for a period of $5 \mathrm{~s}$. West et al. (2011) provides evidence that the IMTP test can be used to measure maximal strength and explosiveness, both of which are related to jump and sprint acceleration performance. The vertical component of the GRF during the IMTP was measured using the portable force platform; the peak force was determined from the vertical component of the GRF-time history during the IMTP minus the participant's body weight (West et al. 2011). The force at $100 \mathrm{~ms}(\mathrm{~F} 100 \mathrm{~ms})$ was defined as the absolute value of the vertical component of the GRF minus the participants body weight $100 \mathrm{~ms}$ after start time. The RFD was taken as the maximum value of the first derivative of the vertical component of the GRF-time history after start time (West et al. 2011).

\subsection{STATISTICAL ANALYSIS}

All data is presented as mean \pm standard deviation (SD). Statistical analyses were carried out using SPSS (SPSS Chicago, IL) with significance being accepted at $\mathrm{p} \leq 0.05$. Following confirmation of parametric assumptions, and tests of sphericity, a time $\mathrm{x}$ trial repeated measures analysis of variance (ANOVA) assessed between-trial differences for variables assessed on multiple occasions per trial at matched time points (i.e., salivary $\mathrm{T}$ and $\mathrm{C}$ ) was complete for chapter 4 . Indices measured only in the experimental trial (i.e. blood lactate, and $\mathrm{T}$ and $\mathrm{C}$ measured pre-and postexercise only) or once per trial (i.e., variables from CMJ and IMTP analyses) were compared using paired t-tests. Post hoc comparisons (Bonferroni adjustments) were made where necessary and effect sizes (ES) were calculated (Cohen 1988). ES were deemed small (0.1), moderate (0.3) and strong (0.5) as per Cohen (1988). Analyses were completed for the combined group, males, females and weightlifting only. Correlations between physiological and performance measures were investigated using Pearson's product-moment correlation coefficients.

For chapter 5 and 6 , following confirmation of parametric assumptions, repeated measures multivariate analysis of variance (MANOVA) with Bonferroni adjustment assessed between-trial differences for variables with multiple time points per trial (i.e. chapter 5: blood lactate, $\mathrm{pH}, \mathrm{sO}_{2} \%$, $\mathrm{PO}_{2}, \mathrm{PCO}_{2}, \mathrm{HCO}_{3}$ and BEecf; chapter 6: T, C, perception muscle soreness, perception recovery, $\mathrm{CK}$, blood lactate, PPO and $\mathrm{JH}$ ). For chapter 5, one-way ANOVA assessed between-trial differences for all performance variables from the swimming TT and RPE recorded post-TT; completed for the combined group, $100 \mathrm{~m}$ and $200 \mathrm{~m}$ also. Smallest worthwhile change in performance was calculated as the difference in performance from the control to $2 \mathrm{~h} \mathrm{IPC}$ and $24 \mathrm{~h}$ IPC. In chapter 6, paired samples t-tests were performed for between-trial comparisons of data expressed over a single time point within a trial (i.e. mean and total sprint times, $\mathrm{T}$ and $\mathrm{C}$ pre-and post-stress test). 


\section{Chapter 4 - The effects of morning exercise with vascular occlusion on afternoon performance}

\subsection{STUDY INFORMATION}

Opportunities exist on the day of competition to influence subsequent performance as athletes may be susceptible to changes in their physical performance as a function of time. Previous research has investigated the use of prior morning exercise to improve afternoon strength performance, associated with an attenuation of $\mathrm{T}$ throughout the day. However, practicalities of implementing previously researched protocols exist and limit application in elite sport and use on the morning of competition. Therefore, alternative strategies are sought after.

Aim: investigate the use of vascular occlusion during resistance exercise in the morning ( $5 \mathrm{~h}$ prior) as a priming strategy to improve afternoon strength/power performance

\section{Objectives:}

- To identify the effect of resistance exercise combined with vascular occlusion on hormone response regarding attenuation of a circadian decline in $\mathrm{T}$

- To identify if there is a difference in $T$ response in male and female athletes

- To identify if an attenuation of $\mathrm{T}$ has a subsequent impact on afternoon performance in male and female athletes

Hypothesis: completing resistance exercise combined with vascular occlusion, performed in the morning, will reduce the decline in $\mathrm{T}$ over $5 \mathrm{~h}$, consequently improving strength/power performance in the afternoon

Outcome measures: T, CMJ, IMTP 


\subsection{INTRODUCTION}

Physical performance can vary over the course of the waking day (Drust et al. 2005; Ammar et al. 2014). Accordingly, circadian rhythmicity of specific physiological responses such as core temperature and endocrine release have been proposed to modulate characteristics deemed key to sport performance (Teo et al. 2011). As competitions commence over a wide range of times (e.g., 11:00 h $-22: 00 \mathrm{~h}$ ), opportunities exist to attenuate circadian-related performance decrements (Chtourou et al. 2012). Therefore, the day of competition has recently been proposed as an opportunity to enhance subsequent performance (Kilduff et al. 2013; Russell et al. 2015).

Testosterone levels are predictive of physical performance (T Crewther et al. 2012); therefore, this biomarker is of interest in athletic performance (Cook \& Crewther 2012; Crewther et al. 2013). In the case of T, circadian rhythmicity displays an early morning (AM) peak before declining (AlDujaili \& Sharp, 2006; Kraemer et al. 2001; Teo et al. 2011) across times corresponding to the start of afternoon/evening (PM) competitions for example over $4 \mathrm{~h}$ a decline of $-10.2 \pm 2.4 \mathrm{pg} \cdot \mathrm{ml}^{-1}$ (Cook et al. 2014) and $-53 \pm 23 \mathrm{pg} \cdot \mathrm{ml}^{-1}$ over $5 \mathrm{~h}$ (Russell et al. 2015) have been reported. Research has highlighted beneficial effects of offsetting a PM decline in T by performing AM priming exercise and enhancing PM performance (Cook et al. 2014; Ekstrand et al. 2012; Russell et al 2015; McGowan et al. 2016; refer to section 2.2.3, Table 2.1). Cook et al. (2014) reported an attenuated decline in $\mathrm{T}$ and improved ( 3\%) lower body strength and power performances $6 \mathrm{~h}$ following an AM training session that incorporated heavy resistance exercise (bench press and back squat routines up to $100 \%$ of $3 \mathrm{RM}$ ) in male rugby union players. Similarly, a $2 \%$ improvement in PM sprint performance occurred following heavy AM upper body resistance exercise (bench press: five sets, 10 repetitions, $75 \%$ one repetition maximum; $1 \mathrm{RM}$ ), also in male rugby union players; a response which corresponded to increased $T$ responses of $+17 \pm 18 \%$ above control values (Russell et al. 2015). Whilst the use of heavy ( $>75 \% 1 \mathrm{RM})$ and relatively high volume (up to 50 repetitions) resistance exercise in the hours preceding subsequent exercise is supported as a means of offsetting the circadian decline in $\mathrm{T}$ (direct stimulatory effect of lactate leading to an increase in T release (Lu et al. 1997) and improving PM performance (Russell et al. 2015; Cook et al. 2014), such approaches may not be practical to pre-competition routines. For example, weightlifters would not favour the completion of the high volume of repetitions previously reported on competition day.

Vascular occlusion is an emerging pre-competition strategy, when combined with resistance exercise, vascular occlusion has demonstrated utility for stimulating training effects with lower volumes (i.e., 25 repetitions at 70\% 1RM [Cook et al. 2014]) of exercise than used in previous 
priming studies (i.e., 50 repetitions at 75\% 1RM [Russell et al. 2015]). Proposed mechanisms (see section 2.5.2) that may benefit pre-competition use include increased metabolic stress (Suga et al. 2012) and muscle fibre recruitment (Takarada et al. 2000; Yasuda et al. 2009). Research identifies a relative intensity and volume threshold must be reached to induce a T response (Cumming et al. 1987), for instance (Ratamess et al. 2005) recognised 6x10 but not 1x10 repetitions of resistance exercise increased $\mathrm{T}$ and thus volume and intensity are required to be sufficient to induce this response. Vascular occlusion is suggested to increase metabolic response due to restricted venous outflow which subsequently enhances the duration of metabolic acidosis via the trapping and accumulation of intramuscular protons $\left(\mathrm{H}^{+}\right)$and stimulation of metabaroceptors possibly eliciting an exaggerated acute systemic hormonal response (Pope et al. 2013). Schoenfeld (2013) reported a greater accumulation of metabolites, due to increased production in a more hypoxic state and limited removal, may act as a primary moderator of anabolic response. Results published by (Cook et al. 2014) support this; the use of vascular occlusion combined with exercise resulted in an occlusion-dependent elevation in salivary $\mathrm{T}$ immediately after exposure.

Acknowledging the practicalities associated with the pre-competition practices of professional athletes, methods of priming PM performances with lower exercise volumes may be better accepted by athletes and coaches on competition day. The use of vascular occlusion may therefore provide an opportunity to reduce the decline in T from AM to PM and contribute to improved PM performance, using lower volumes of resistance exercise than examined previously in AM priming studies (Russell et al. 2015; Cook et al. 2014). Therefore, this study sought to ascertain whether, in comparison to a passive rest control condition (Con), completion of AM resistance exercise with vascular occlusion (RE+VO) elicits improved PM performance in field athletics and weightlifting athletes. It was hypothesized that the inclusion of morning $\mathrm{RE}+\mathrm{VO}$ would reduce the decline in $\mathrm{T}$ from $\mathrm{AM}$ to $\mathrm{PM}$ and thus transfer to improved PM performance.

In addition to the primary aim, previous research into morning priming and the $\mathrm{T}$ response to vascular occlusion have been predominately completed in male participants including circadian rhythmicity research is sport. Ekstrand et al. (2012) and Ingham et al. (2013) investigated AM exercise on PM performance in both male and females, identifying a positive response, however results were reported as a group, not distinguishing between sexes and hormonal responses were not measured. Specifically regarding circadian rhythmicity, Kraemer et al. (2001) investigated the effect of heavy resistance exercise on the circadian rhythm of salivary $\mathrm{T}$ in men and Hatfield et al. (2016) examined the effects of circadian rhythm on power, force and hormonal response in young men. To date, no investigation exists regarding the use of AM exercise in females and limited research exists in elite athlete, female response to exercise and circadian rhythmicity. Cook et al. (2012) and Cook \& Beaven (2013) provide two investigations in female athletes; comparison of 
baseline free $\mathrm{T}$ and $\mathrm{C}$ concentrations between elite and non-elite female athletes and salivary $\mathrm{T}$ is related to self-selected training load in elite female athletes. Findings suggesting elite female athletes have higher baseline $\mathrm{T}$ levels than non-elite females and secondly individual $\mathrm{T}$ concentrations demonstrated strong relationships with self-selected workloads during training, similar to results reported in male participants (Cook \& Beaven 2013). This evidence suggests AM exercise may improve performance and hormone response as identified in male counterparts, however, no previous research exists in females or specifically with female athletes. Therefore, this study also aimed to provide novel research on the effect of AM exercise on PM performance and diurnal changes in $\mathrm{T}$ concentration in female athletes.

\subsection{METHOD}

\subsubsection{Experimental overview}

In a group of internationally competitive athletes, the effects of AM exercise completed prior to PM exercise performance were investigated using a crossover, randomised control trial. The study consisted of two interventions; Con and RE+VO. In both conditions, AM and PM ( 9.00 h and $\sim 14.00 \mathrm{~h})$ saliva samples were provided to assess hormone responses. The AM procedures consisted of participants randomly assigned to the Con or $\mathrm{RE}+\mathrm{VO}$, followed by $\sim 5 \mathrm{~h}$ rest until PM measures of CMJ and IMTP were completed. The RE+VO condition consisted of five sets of five repetitions of back squat at 70\% 1RM with bi-lateral occlusion cuffs placed at the most proximal point of the thigh inflated to an individualised cuff pressure that was determined beforehand from thigh girth, and systolic and diastolic blood pressures. Testing procedures were completed at a consistent time to limit circadian variation and were separated by seven days.

\subsubsection{Participants}

Following ethical approval and informed consent being attained, 22 international standard athletic (pole vault and high jump) and weightlifting athletes were recruited through the Welsh Institute of Sport (10 males, 12 females; Age: $24 \pm 6$ years, mass: $74 \pm 15 \mathrm{~kg}$, stature: $168 \pm 14 \mathrm{~cm}$, competition level: Welsh International; training history: $5 \pm 4$ years; training 1-2 times daily, $6 \mathrm{x}$ week; refer to Table 4.1 for male and female split), completed the study protocol. Testing was conducted in the participant's maintenance phase of training and rested and abstained from alcohol for $24 \mathrm{~h}$ before each main trial. As part of consent procedures, female participants were asked about current hormone contraceptive use, of which three participants were currently using oral contraceptive pills; all other female participants reported regular menstrual cycling, determined according to section 3.2 . 


\subsubsection{Main trial procedures}

Upon arrival for each main trial ( 09:00 h), participants rested for $10 \mathrm{~min}$ before blood pressure and thigh girth measurements were taken (Omron Healthcare, Europe; systolic $>140 \mathrm{mmHg}$ and/or diastolic $>90 \mathrm{mmHg}$ precluded further study involvement) (section 3.3). A baseline saliva sample was collected before the experimental interventions and female participants provided detail on the stage of menstrual cycle. A $5 \mathrm{~h}$ passive rest period followed, during which unnecessary ambulation was minimised. Thereafter, PM $(\sim 14: 00 \mathrm{~h})$ assessments of salivary hormones and physical performance tests (CMJ and IMTP) were completed.

For RE+VO, participants provided a $20 \mu \mathrm{L}$ fingertip capillary blood sample to determine baseline values (analysed retrospectively for lactate concentrations; Biosen C-Line Clinic, EKF Diagnostic GmgH, Barleben, Germany) before undertaking a standardised warm-up (5-min cycle, six sets of back squat from 30-65\% 1RM). Following 5-min of recovery, five sets of five repetitions of back squat (70\% 1RM, $60 \mathrm{~s}$ intra-set recovery) (Cook et al. 2014) were performed while wearing $11 \mathrm{~cm}$ wide occlusion cuffs (Sports Rehab Tourniquet, Sportsrehab) on the most proximal point of each thigh. Immediately prior to each set, cuffs were inflated to $60 \%$ of individually calculated pressures (147-240mmHg) (Table 4.2), before being deflated during recovery. Individual pressures were calculated from thigh girth and resting blood pressure measurements and calculated in accordance with Loenneke et al. (2015) (see section 3.4). Immediately after the final set, saliva and capillary blood samples were collected. Such procedures took $\sim 30 \mathrm{~min}$ to perform. For Con, no morning exercise was undertaken, participants presented themselves for baseline measures only and were instructed to remain seated for $\sim 30$ min whilst the RE+VO were undertaking the intervention. All participants returned after $5 \mathrm{~h}$ rest period for PM measures, a saliva sampled was completed first, prior to CMJ and IMTP.

Saliva collection was completed as detailed in Chapter 3, section 3.7. Assessment of CMJ and IMTP performance was completed using a force platform (type 92866AA, Kistler Instruments Ltd., Farnborough, UK), in accordance with the described methodology specified in Chapter 3, section 3.8, PPO, JH and peak force were calculated. 


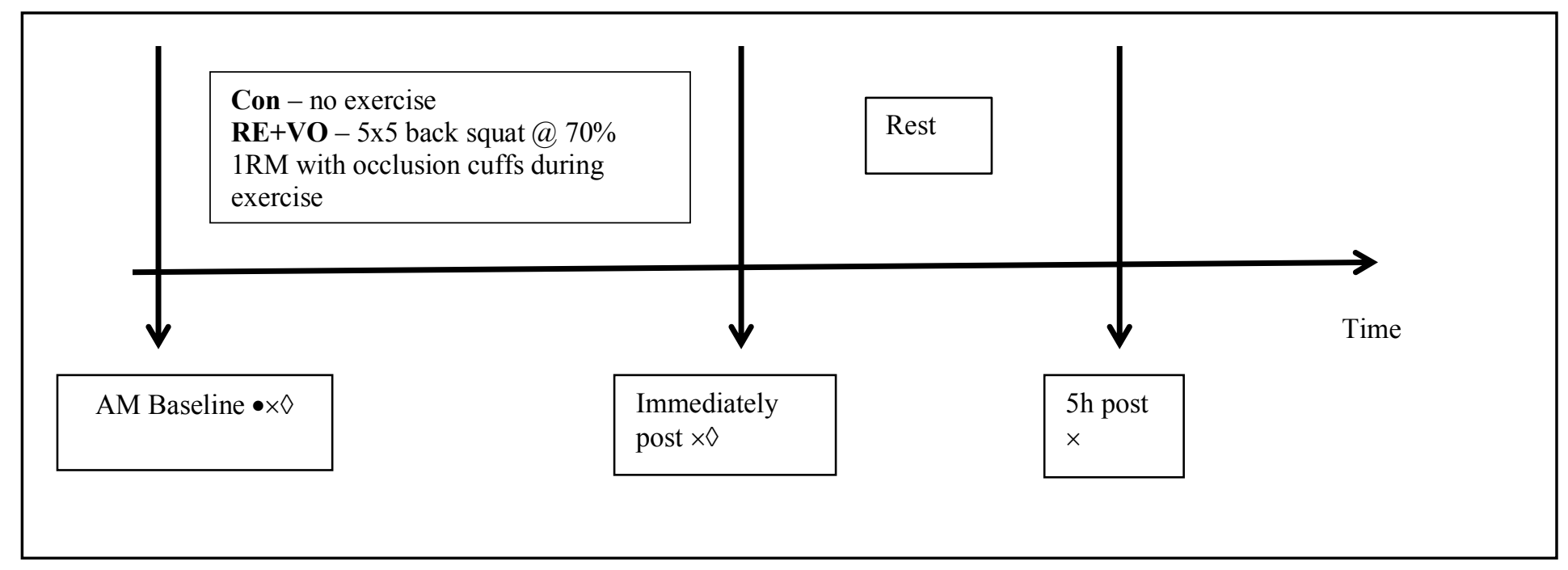

Figure 4.1: Timeline of data collection. Con; control: RE+VO; resistance exercise with vascular occlusion: RM; repetition maximum. Measurements: blood pressure $(\bullet)$, testosterone and cortisol $(\times)$, blood lactate $(\diamond)$, Countermovement jump \& isometric mid-thigh pull ( ) 
Table 4.1: Participant characteristics

\begin{tabular}{lll}
\hline Baseline Characteristics & Males & Females \\
\hline Age (yrs) & $21.2 \pm 3.8$ & $26.2 \pm 5.8$ \\
Height $(\mathrm{cm})$ & $188.4 \pm 9.2$ & $161.9 \pm 8.1$ \\
Weight $(\mathrm{kg})$ & $85.3 \pm 10.2$ & $63.9 \pm 9.4$ \\
Resting systolic blood pressure & $138.1 \pm 13.3$ & $121.4 \pm 10.6$ \\
(mmHg) & \\
Thigh girth (cm) & $61.7 \pm 4.3$ & $57.8 \pm 4.6$ \\
Training history (yrs) & $3.9 \pm 2.9$ & $5.9 \pm 4.9$ \\
Cuff Pressure (mmHg) & $218.1 \pm 12.2$ & $189.2 \pm 24.0$
\end{tabular}

Values are expressed as Mean \pm standard deviation 


\subsubsection{Statistical analysis}

All data is presented as mean $\pm \mathrm{SD}$. Following confirmation of parametric assumptions and sphericity, a time $\mathrm{x}$ trial repeated ANOVA assessed between-trial differences for variables assessed on multiple occasions per trial at matched time points (i.e., salivary $\mathrm{T}$ and $\mathrm{C}$ ) was complete. Indices measured only in $\mathrm{RE}+\mathrm{VO}$ trial (i.e. blood lactate, and $\mathrm{T}$ and $\mathrm{C}$ measured preand post-exercise only) or once per trial (i.e., variables from CMJ and IMTP analyses) were compared using paired t-tests. Correlations between physiological and performance measures were investigated using Pearson's product-moment correlation coefficients. Where appropriate results were analysed in homogenous groups e.g. males and females. Please refer to Chapter 3 , section 3.9 for more detail. 
Table 4.2: Individual cuff pressures

\begin{tabular}{ll}
\hline Individual cuff pressures $(\mathrm{mmHg})$ & \\
\hline Male Participants & Female Participants \\
\hline 194.1 & 172.2 \\
227 & 161.9 \\
192.7 & 195 \\
240 & 200 \\
219.8 & 200 \\
220 & 163.9 \\
210.9 & 147.7 \\
219 & 185 \\
220 & 217 \\
223.9 & 206.7 \\
& 220 \\
& 206.6 \\
\hline $216.7 \pm 14.4$ & $189.7 \pm 23.4$ \\
\hline
\end{tabular}




\subsection{RESULTS}

\subsubsection{Combined group results}

Salivary $\mathrm{T}$ concentrations (Figure 4.2) were influenced by condition (Time $\mathrm{x}$ trial interaction: $\left.\mathrm{F}_{(1,20)}=9.658, \mathrm{p}=0.006\right)$ and time of sample (Time effect: $\left.\mathrm{F}_{(1,20)}=25.537, \mathrm{p}<0.001\right)$. Following paired samples analysis; although pre-exercise differences were observed (Con; $120.43 \pm 40.42$ $\left.\mathrm{pg} \cdot \mathrm{ml}^{-1} 1, \mathrm{RE}+\mathrm{VO} ; 101.94 \pm 31.30 \mathrm{pg} \cdot \mathrm{ml}^{-1}, \mathrm{p}=0.012, \mathrm{ES}=0.51\right)$ the delta values $(\mathrm{p}=0.006, \mathrm{ES}=0.89)$ in $\mathrm{T}$ from $\mathrm{AM}$ to $\mathrm{PM}$ in $\mathrm{Con}\left(-32.02 \pm 28.32 \mathrm{pg} \cdot \mathrm{ml}^{-1},-23.6 \pm 19.9 \%, \mathrm{p}<0.001, \mathrm{ES}=0.94\right)$ was larger than the decrease in $\mathrm{RE}+\mathrm{VO}\left(-9.60 \pm 21.77 \mathrm{pg} \cdot \mathrm{ml}^{-1},-5.8 \pm 22.1 \%, \mathrm{p}=0.086, \mathrm{ES}=0.33\right)$. Post exercise $\mathrm{T}$ following RE+VO condition increased significantly $\left(+18.51 \pm 32.52 \mathrm{pg} \cdot \mathrm{ml}^{-1},+10.7 \pm\right.$ $21.5 \%, \mathrm{p}=0.014, \mathrm{ES}=0.45$ ).

Salivary C concentrations demonstrated significant time $\left(\mathrm{F}_{(1,21)}=39.983, \mathrm{p}<0.001\right)$ but not time $\mathrm{x}$ trial interaction effects $\mathrm{AM}$ to $\mathrm{PM}\left(\mathrm{F}_{(1,21)}=2.765, \mathrm{p}=0.111\right)$. Measured in $\mathrm{RE}+\mathrm{VO}$ only, a significant post-exercise increases in $\mathrm{C}$ occurred $\left(+0.094 \pm 0.16 \mathrm{ug} \cdot \mathrm{dL}^{-1},+29.8 \pm 43.5 \%, \mathrm{p}=0.014\right.$, $\mathrm{ES}=0.48)$.

Blood lactate concentrations increased post exercise in $\mathrm{RE}+\mathrm{VO}\left(5.03 \pm 2.7 \mathrm{mmol} \cdot \mathrm{l}^{-1}, 78.9 \pm 13.6 \%\right.$, $\mathrm{p}<0.001, \mathrm{ES}=2.94$ ). The delta changes in blood lactate and $\mathrm{T}$ concentrations pre-to post exercise showed a significant, large positive correlation $(\mathrm{r}=0.559, \mathrm{p}=0.007)$ (Figure 4.3). The $\Delta \mathrm{T}$ values from pre- to post-exercise were significantly correlated with occlusion cuff pressure $(\mathrm{r}=0.437$, $\mathrm{p}=0.042$ ). The data also identified a significant positive correlation between $\Delta$ blood lactate concentration and occlusion cuff pressure in $\mathrm{RE}+\mathrm{VO}(\mathrm{r}=0.423, \mathrm{p}=0.05)$.

Performance measures are presented in Table 4.3 and 4.4. There were no significant differences in CMJ PPO ( $p=0.832), J H(p=0.422)$ or $\mathrm{V}_{\max }(\mathrm{p}=0.445)$ between conditions, nor were there any significant differences in IMTP peak force $(p=0.120)$, F100ms $(p=0.734)$ and RFD $(p=0.802)$. However, there were large individual differences in IMTP peak force (2 to 17\%) (Figure 4.5). There were no significant relationships between the percentage changes in $\mathrm{T}$ concentrations from AM to PM in the occlusion trial and the percentage change in CMJ PPO $(p=0.750)$ or IMTP peak force $(p=0.119)$. However, a significant correlation was observed between change in $T$ concentration from pre-to post exercise and the difference in IMTP peak force between conditions $(\mathrm{r}=0.488, \mathrm{p}=0.021)$. 
(A)
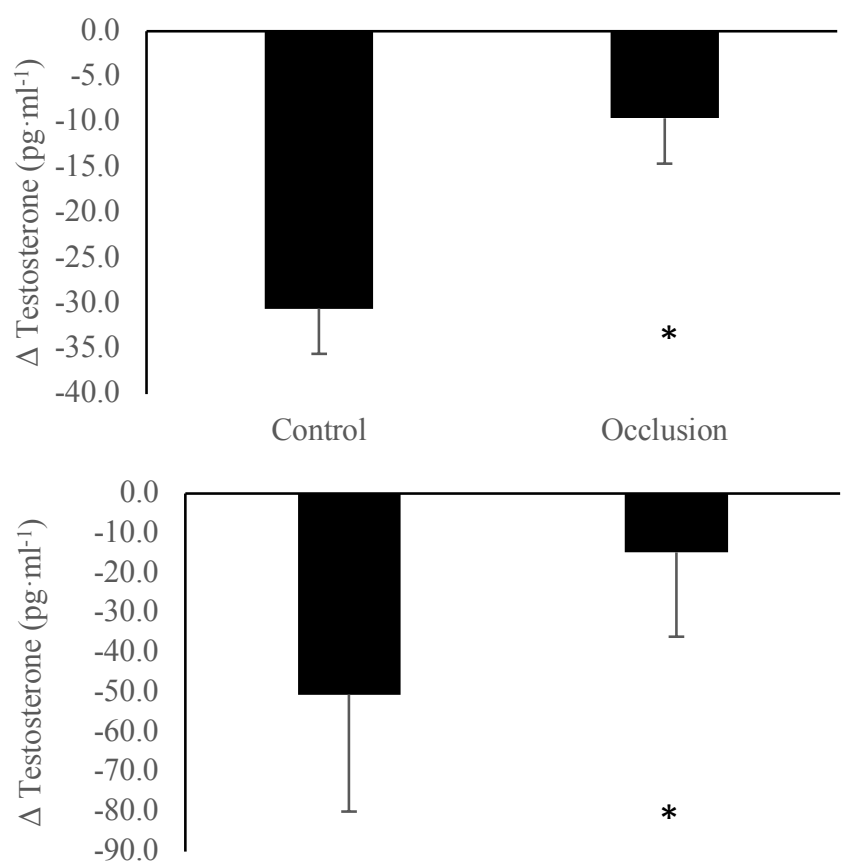

(B)

(C)

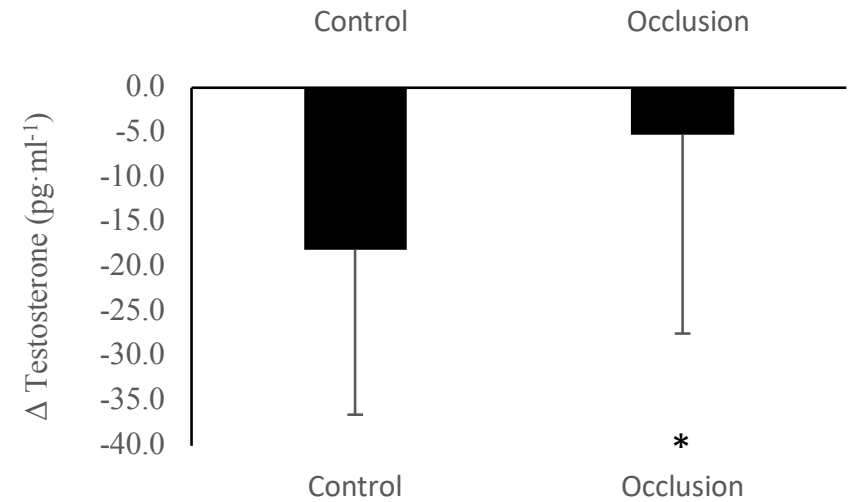

(D)

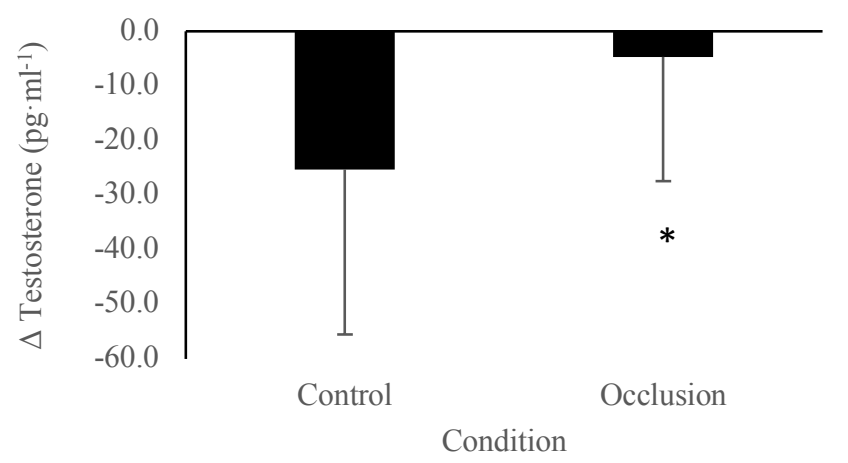

Figure 4.2: $\Delta$ Testosterone concentrations (mean $\pm \mathrm{SD}$ ) throughout the control (Con) and resistance exercise with vascular occlusion (RE+VO) condition. (A) All participants, (B) male participants only, (C) female participants only, (D) weightlifting only participants * indicates significant $(\mathrm{p}<0.05)$ delta change measured AM to PM. 


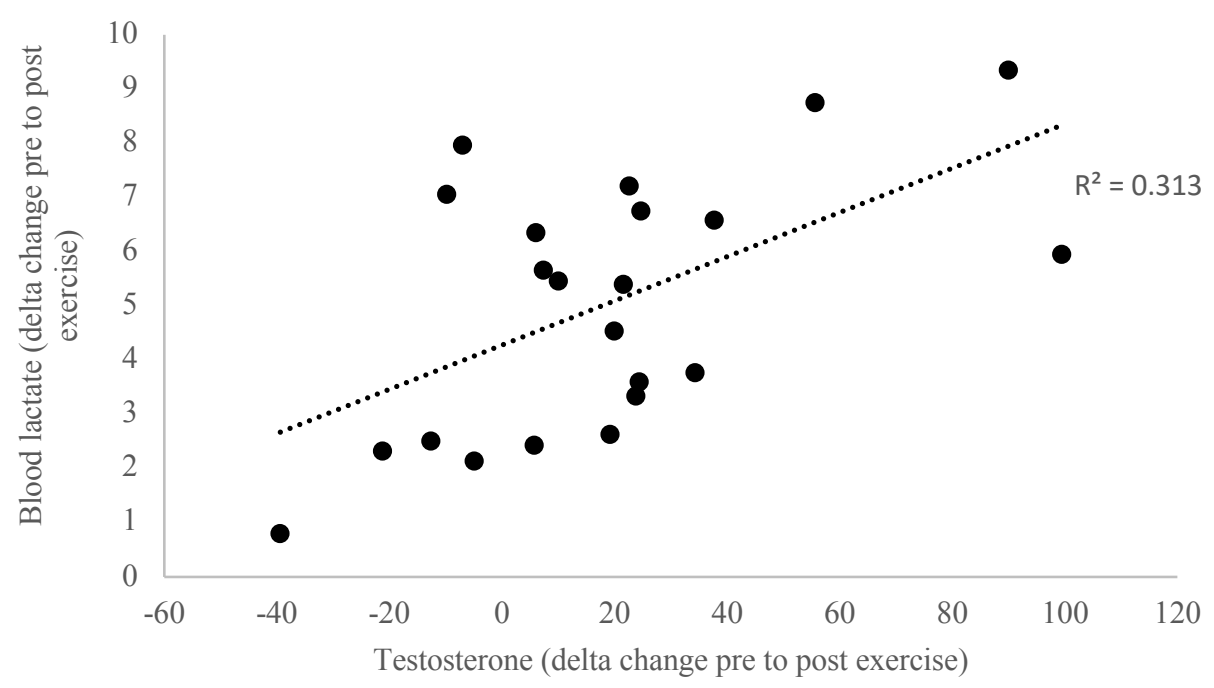

Figure 4.3: Correlation between the change in blood lactate and Testosterone pre-to postexercise 
(A)

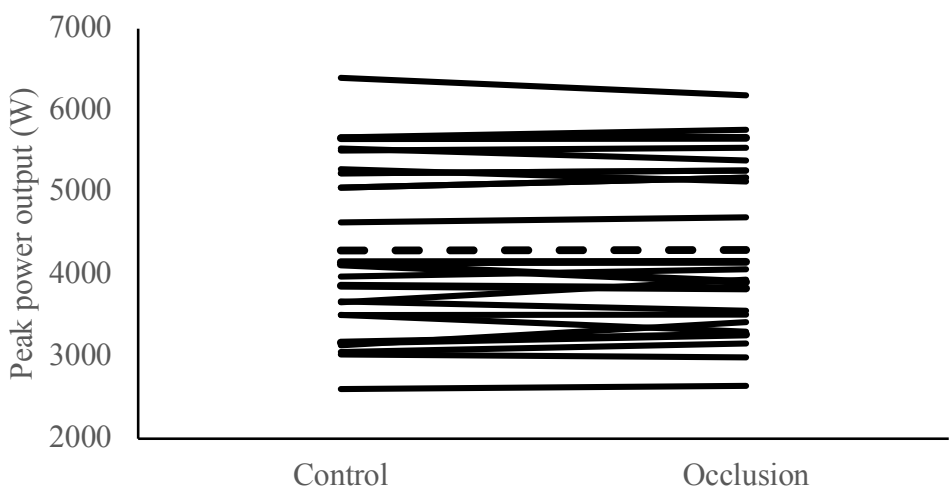

(B)

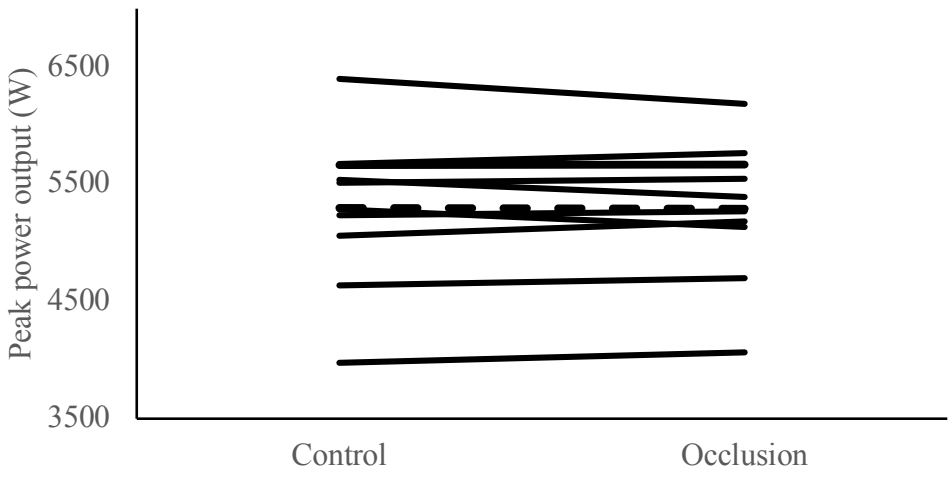

(C)

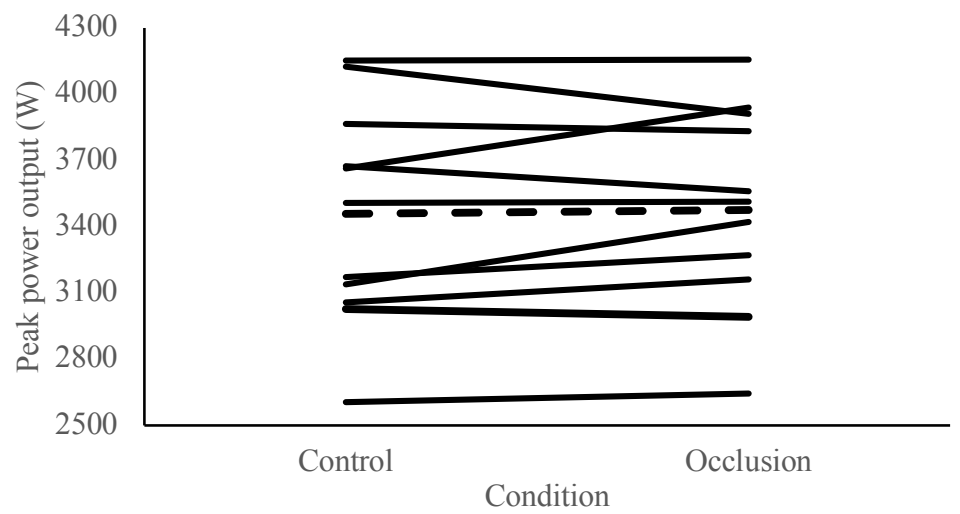

Figure 4.4: Individual response in performance of peak power output determined from countermovement jump for the control and occlusion conditions. (A) All participants, (B) male participants, (C) female participants 
(A)

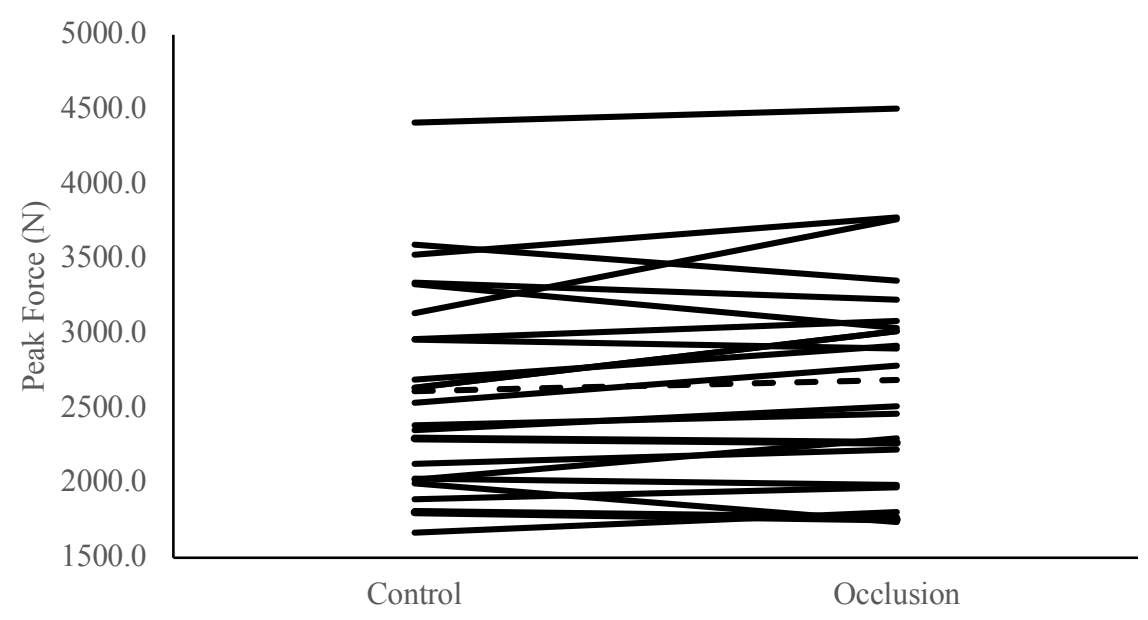

(B)

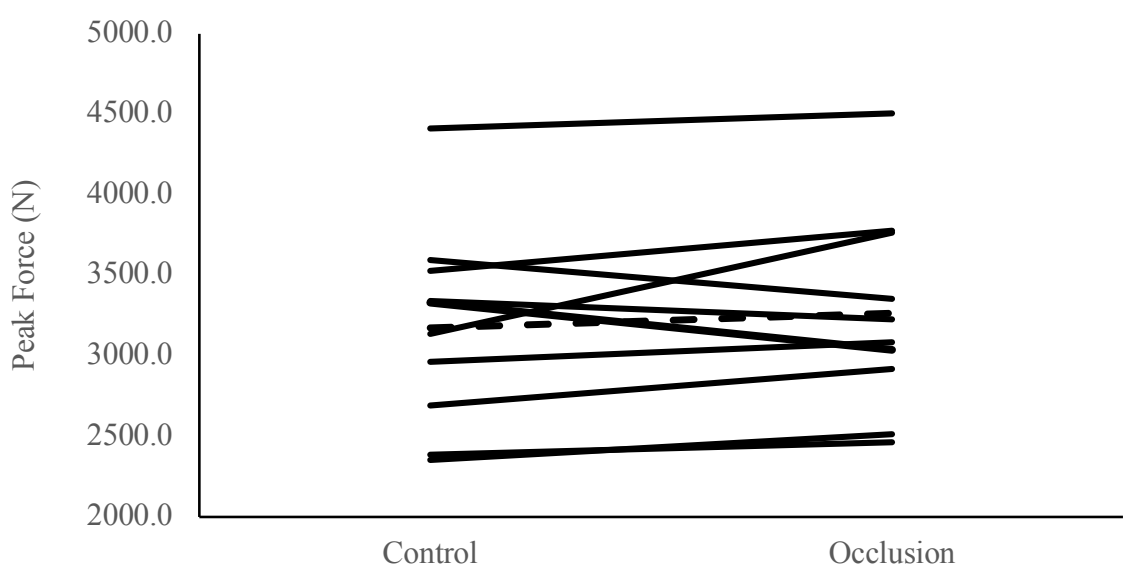

(C)

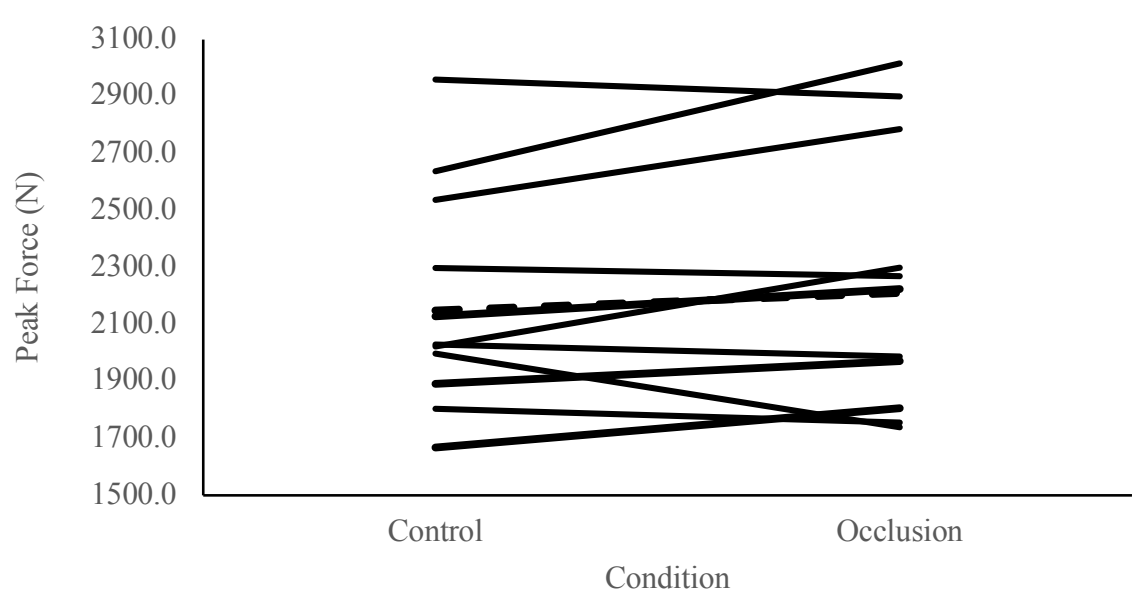

Figure 4.5: Individual response in performance of peak force determined from isometric midthigh pull for the control and occlusion conditions. (A) All participants, (B) male participants, (C) female participants 


\subsubsection{Male participants}

In male participants, $\mathrm{T}$ concentrations were influenced by condition (Time $\mathrm{x}$ trial interaction; $\left.\mathrm{F}_{(1,8)}=7.040, \mathrm{p}=0029\right)$ and time of sample (Time effect; $\mathrm{F}_{(1,8)}=42.409, \mathrm{p}<0.001$ ) (Figure 4.2). Testosterone concentrations declined significantly from AM to PM in the control trial $(-50.55 \pm$ $\left.29.39 \mathrm{pg} \cdot \mathrm{ml}^{-1},-32.2 \pm 15.3 \%, \mathrm{p}=0.001\right)$, displaying very large effect sizes $(\mathrm{ES}=1.50)$, but not following RE+VO $\left(-14.78 \pm 21.21 \mathrm{pg} \cdot \mathrm{ml}^{-1},-10.9 \pm 17.2 \%, \mathrm{p}=0.110, \mathrm{ES}=0.59\right)$.

Although T concentrations were higher after completion of $\mathrm{RE}+\mathrm{VO}$, this did not reach significance $\left(+27.94 \pm 44.14 \mathrm{pg} \cdot \mathrm{ml}^{-1},+13.6 \pm 26.3 \%, \mathrm{p}=0.076\right)$ despite a medium to large effect size $(\mathrm{ES}=0.74)$. Blood lactate was significantly higher following RE+VO $\left(6.37 \pm 2.52 \mathrm{mmol} \cdot 1^{-1}, 80.9 \pm 16.55\right.$, $\mathrm{p}<0.001$ ) with a positive significant correlation between the change in blood lactate and occlusion cuff pressure $(\mathrm{r}=0.722, \mathrm{p}=0.018)$. The change in blood lactate did not correlate with the change in $\mathrm{T}$ concentration $(\mathrm{r}=0.522, \mathrm{p}=0.122)$.

Salivary $\mathrm{C}$ concentrations were not affected by condition (Time $\mathrm{x}$ trial interaction; $\mathrm{F}_{(1,9)}=0.289$, $p=0.604)$, but there was a significant effect of time of measurement $\left(F_{(1,9)}=25.843, p<0.001\right)$ in response to trials. Cortisol concentrations decreased from AM to PM in both the control $(-0.257$ $\left.\pm 0.18 \mathrm{ug} \cdot \mathrm{dL}^{-1},-49.9 \pm 21.1 \%, \mathrm{p}=0.001, \mathrm{ES}=1.62\right)$ and $\mathrm{RE}+\mathrm{VO}\left(-0.304 \pm 0.26 \mathrm{ug} \cdot \mathrm{dL}^{-1},-51.3 \pm\right.$ $18.6 \%, \mathrm{p}=0.005, \mathrm{ES}=1.50$ ) conditions.

Neither conditions had an effect on any performance measures reported in Table 4.3 and 4.4 $(p<0.05)$. However, as displayed in figure 4.4 and 4.5 there was individual variation in performance. There were no significant associations between percentage change in $T$ concentrations from AM to PM in the occlusion trial and percentage change in CMJ PPO $(p=0.864)$ or IMTP peak force $(\mathrm{p}=0.630)$.

\subsubsection{Female participants}

In female only participants, $\mathrm{T}$ concentrations were not affected by condition (Time $\mathrm{x}$ trial interaction; $F_{(1,11)}=3.452 \mathrm{p}=0.090$ ), but did vary due to sampling time (Time effect; $F_{(1,11)}=6.035$, $\mathrm{p}=0.032$ ) (Figure 4.2). Testosterone concentrations declined from AM to PM in the control condition $\left(-18.12 \pm 18.41 \mathrm{pg} \cdot \mathrm{ml}^{-1},-17.2 \pm 21.1 \%\right.$, $\left.\mathrm{p}=0.006, \mathrm{ES}=0.76\right)$, however results did not significantly decline following $\mathrm{RE}+\mathrm{VO}\left(-5.28 \pm 22.18 \mathrm{pg} \cdot \mathrm{ml}^{-1},-1.6 \pm 25.5 \%, \mathrm{p}=0.427, \mathrm{ES}=0.21\right)$.

There were significant differences in $\mathrm{T}$ concentration pre-to post $\mathrm{RE}+\mathrm{VO}\left(+10.65 \pm 16.64 \mathrm{pg} \cdot \mathrm{ml}^{-1}\right.$, $+8.3 \pm 17.5 \%, \mathrm{p}=0.049, \mathrm{ES}=0.34)$. Blood lactate also significantly increased pre-to post RE+VO 
$\left(3.92 \pm 1.59 \mathrm{mmol} \cdot \mathrm{l}^{-1}, 77.2 \pm 11.0 \%, \mathrm{p}<0.001\right)$. However, there was no significant correlation between the change in $\mathrm{T}$ concentration and the change in blood lactate $(\mathrm{r}=0.521, \mathrm{p}=0.083)$.

Salivary $\mathrm{C}$ concentration was significantly affected by condition (Time $\mathrm{x}$ trial interaction; $\left.\mathrm{F}_{(1,11)}=8.654, \mathrm{p}=0.013\right)$ and time of sampling $\left(\mathrm{F}_{(1,11)}=15.928, \mathrm{p}=0.002\right)$. Cortisol concentrations declined from AM to PM under the control $\left(-0.381 \pm 0.32 \mathrm{ug} \cdot \mathrm{dL}^{-1}, 60.9 \pm 17.9 \%, \mathrm{p}=0.002\right.$, $\mathrm{ES}=1.39)$ and $\mathrm{RE}+\mathrm{VO}\left(-0.143 \pm 0.20 \mathrm{ug} \cdot \mathrm{dL}^{-1}, 30.2 \pm 36.9 \%, \mathrm{p}=0.027, \mathrm{ES}=0.84\right)$ condition.

There were no significant differences in any performance measures reported in Table 4.3 and 4.4, for example CMJ PPO $(\mathrm{p}=0.709)$ and IMTP peak force $(\mathrm{p}=0.254)$. There was also no significant relationship between percentage change in T from AM to PM in occlusion trial and the percentage change in CMJ PPO $(p=0.776)$ or IMTP peak force $(p=0.124)$.

\subsubsection{Weightlifting participants}

Salivary $T$ concentrations were influenced by condition $\left(F_{(1,17)}=25.264 ; p<0.001\right)$ and time of sample $\left(\mathrm{F}_{(1,17)}=0.278, \mathrm{p}<0.001\right)$ (Figure 4.2). There was a significant decline in $\mathrm{T}$ from $\mathrm{AM}$ to $\mathrm{PM}$ in the control $\left(-25.27 \pm 30.25 \mathrm{pg} \cdot \mathrm{ml}^{-1},-16.2 \pm 21.3 \%, \mathrm{p}<0.001\right)$, larger than the decrease identified in RE+VO $\left(-4.67 \pm 22.77 \mathrm{pg} \cdot \mathrm{ml}^{-1},-1.1 \pm 21.7 \%\right)$. A significant difference was displayed between the decline in T from AM to PM in the control compared to RE+VO $(p=0.024)$. Post exercise $\mathrm{T}$ following $\mathrm{RE}+\mathrm{VO}$ increased significantly $\left(+22.0 \pm 27.9 \mathrm{pg} \cdot \mathrm{ml}^{-1},+13.9 \pm 16.2 \%\right.$, $\mathrm{p}=0.031)$.

Blood lactate significantly increased following exercise $(\mathrm{p}<0.001)$ and was significantly correlated to the $\Delta \mathrm{T}$ pre-to post exercise $(\mathrm{r}=0.475, \mathrm{p}=0.047)$.

Salivary C concentrations were not affected by condition $\left(\mathrm{F}_{(1,17)}=0.398, \mathrm{p}=0.537\right)$ but was affected by time of sample $\left(\mathrm{F}_{(1,17)}=33.371, \mathrm{p}<0.001\right)$.

An increase in CMJ PPO was observed $(0.6 \pm 3.5 \%)$, although consistent with other results this did not reach significance ( $\mathrm{p}=0.507$ ), similarly for $\mathrm{JH}$ an increase was observed following RE $+\mathrm{VO}$ $(0.9 \pm 4.6 \%)$ but was not significantly different between conditions $(p=0.329)$ and $\mathrm{V}_{\max }(\mathrm{p}=0.408)$. Peak force from IMTP was not significantly different between conditions $(\mathrm{p}=0.069)$, despite an increase of $3.2 \pm 7.7 \%$, this was consistent for F@100ms ( $\mathrm{p}=0.965)$ and RFD $(\mathrm{p}=0.850)$. 
Table 4.3: Performance outcomes from countermovement jumps for the control and vascular occlusion conditions

\begin{tabular}{lllll}
\hline Condition & Participants & Performance & & \\
\cline { 3 - 5 } & & CMJ PPO (W) & Jump Height $(\mathrm{cm})$ & $\mathrm{V}_{\max }\left(\mathrm{m}_{\mathrm{s}}{ }^{-1}\right)$ \\
\hline Control & Group & $4295.0 \pm 1086.0$ & $37.5 \pm 5.6$ & $2.8 \pm 0.2$ \\
Vascular Occlusion & Group & $4301.4 \pm 1051.8$ & $37.8 \pm 5.6$ & $2.8 \pm 0.2$ \\
\hline Percentage change & & $0.3 \pm 0.0 \%$ & $0.7 \pm 4.4 \%$ & $0.3 \pm 2.1 \%$ \\
\hline Control & Males & $5299.1 \pm 652.9$ & $36.7 \pm 9.0$ & $3.0 \pm 0.1$ \\
Vascular Occlusion & Males & $5292.1 \pm 590.2$ & $36.8 \pm 9.0$ & $3.0 \pm 0.1$ \\
\hline Percentage change & & $0.0 \pm 2.2 \%$ & $0.3 \pm 4.0 \%$ & $-0.3 \pm 2.0 \%$ \\
\hline Control & Females & $3458.2 \pm 471.4$ & $35.7 \pm 8.1$ & $2.7 \pm 0.2$ \\
Vascular occlusion & Females & $3475.8 \pm 437.2$ & $36.2 \pm 8.2$ & $2.7 \pm 0.2$ \\
\hline Percentage change & & $0.5 \pm 4.4 \%$ & $1.5 \pm 4.0 \%$ & $0.9 \pm 2.0 \%$ \\
\hline
\end{tabular}

Mean \pm standard deviation. CMJ - countermovement jump; PPO - peak power output; $\mathrm{V}_{\max }$ - peak velocity 
Table 4.4: Performance outcomes from isometric mid-thigh pull for the control and vascular occlusion conditions

\begin{tabular}{|c|c|c|c|c|}
\hline \multirow[t]{2}{*}{ Condition } & \multirow[t]{2}{*}{ Participants } & \multicolumn{3}{|l|}{ Performance } \\
\hline & & Peak force $(\mathrm{N})$ & F100ms (N) & $\operatorname{RFD}\left(\mathrm{N} \cdot \mathrm{s}^{-1}\right)$ \\
\hline Control & Group & $2614.9 \pm 718.6$ & $711.4 \pm 345.1$ & $12379.1 \pm 5552.1$ \\
\hline Vascular Occlusion & Group & $2689.9 \pm 752.6$ & $773.0 \pm 378.4$ & $13315.5 \pm 5502.9$ \\
\hline Percentage change & & $2.3 \pm 7.6 \%$ & $0.8 \pm 38.6 \%$ & $1.6 \pm 32.3 \%$ \\
\hline Control & Males & $3174.0 \pm 619.9$ & $920.3 \pm 215.6$ & $16113.3 \pm 5611.8$ \\
\hline Vascular Occlusion & Males & $3265.0 \pm 621.1$ & $1027.3 \pm 290.6$ & $17343.4 \pm 4901.8$ \\
\hline Percentage change & & $2.6 \pm 7.8 \%$ & $1.9 \pm 39.2 \%$ & $2.0 \pm 32.0 \%$ \\
\hline Control & Females & $2148.9 \pm 387.6$ & $521.4 \pm 336.2$ & $9578.4 \pm 3630.1$ \\
\hline Vascular occlusion & Females & $2210.7 \pm 463.1$ & $541.8 \pm 294.7$ & $10294.7 \pm 3788.7$ \\
\hline Percentage change & & $2.0 \pm 7.8 \%$ & $0.1 \pm 39.8 \%$ & $1.3 \pm 34.0 \%$ \\
\hline
\end{tabular}

Mean \pm standard deviation. F100ms - force at $100 \mathrm{~ms}$, RFD - rate of force development 


\subsection{DISCUSSION}

This study examined the influence of combining morning exercise with vascular occlusion on afternoon performance and associated hormonal changes of internationally competitive field athletics and weightlifting athletes. Although no combined group performance effects were observed, AM exercise combined with vascular occlusion attenuated the AM to PM decline in salivary $\mathrm{T}$ concentrations over a $5 \mathrm{~h}$ period and caused an acute increase in $\mathrm{T}$ concentrations postexercise. No significant effects were observed for $\mathrm{C}$ concentrations from AM to PM. The results displayed mainly consistent patterns when males, females and weightlifting only groups were analysed. Such findings suggest the use of resistance exercise combined with vascular occlusion as a method of attenuating the decline in $\mathrm{T}$, but further research is required to determine its potential efficacy for performance following the individual responses observed (Figures 4.4 and 4.5).

Completing AM exercise has formerly been demonstrated to be beneficial to reduce a circadian decline in T over the day and consequently positively impact afternoon performance (Russell et al. 2015; Cook et al. 2014). Consistent with previous results (Russell et al. 2015; Cook et al. 2014), the current study identified a decline in $\mathrm{T}$ by $-32.02 \pm 28.32 \mathrm{pg} \cdot \mathrm{ml}^{-1}(-23.6 \pm 19.9 \%)$ between 09:00 $\mathrm{h}$ and 14:00 $\mathrm{h}$ in the control. Conversely, this decline was attenuated by performing RE+VO, where reductions of $-9.60 \pm 21.77 \mathrm{pg} \cdot \mathrm{ml}^{-1}(-5.8 \pm 22.1 \%)$ were observed. Testosterone has previously been associated with quality of work performed by regulating $\mathrm{Ca}^{2+}$ handling, muscle contractility and motor cortex outputs, further contributing to human performance through its putative actions on mood, behaviour and cognition (Crewther et al. 2012). Therefore, $T$ can have a positive impact on performance and as such has received signficiant attention on methods of increasing $\mathrm{T}$ response and also preventing circadian decline in $\mathrm{T}$ throughout the day to enable afternoon performance to be completed with higher $\mathrm{T}$ levels. Reagrdng the acute response, previous research has discussed the requirement of high intensity and volume thresholds to be reached in order to achieve a hormone (T) response with examples from Ratamess et al. (2005) suggesting $6 \times 10$ repetitions but not $1 \times 10$ resulted in a $\mathrm{T}$ response. This has been linked to the metabolic accumulation required to induce a $\mathrm{T}$ response. Consequently, previous research implemting morning exercise to influence afternoon $\mathrm{T}$ and performance have implemented high volumes of resistance exercise (e.g. 5x10 bench press; Russell et al 2015), however, this lacks practicality for completion the day of competition especially for particular sports such as weightlifting. The use of combining resistance exercise and vascular occlusion was hypothesized to attentuate the circadian decline in $\mathrm{T}$, due to mechanisms associated with increased metabolic accumulation, the result of decreased venous outflow and conquently increasing hormone response with a lower volume and intensity of resistance exercise, which was achieved. 
Unfrotunately, intramsuclar measurements of muscle lactate, $\mathrm{H}^{+}$, ATP and $\mathrm{PCr}$ concentrations were not possible in this study to delineate mechanisms resulting in attentuated $\mathrm{T}$ concentrations. Nevertheless, blood lactate data, as also completed by (Teixeira et al. 2017), provided a superficial insight into the metabolic response to RE+VO. Notably, the $78.9 \pm 13.6 \%$ increase in blood lactate concentrations that occurred immediately after RE+VO exposure, when the whole group was analysed, correlated positively ( $\mathrm{r}=0.559)$ with $\Delta \mathrm{T}$ concentrations (i.e., $10.7 \pm 21.5 \%$; pre-to post exercise). As maximal exercise elicits a rapid endocrine response that normally relates to exercise intensity (Buśko \& Opaszowski 2005), and correlations between blood lactate and T changes exist following heavy resistance exercise (Kraemer et al. 1991), a direct stimulatory effect of lactate may lead to increased release of $\mathrm{T}$ ( $\mathrm{Lu}$ et al. 1997). Notably, the magnitude of change in $\mathrm{T}$ immediately after $\mathrm{RE}+\mathrm{VO}$ was equivocal of those seen following acute androgenic stimulating activities (Cook et al. 2014). These results are supported by Cook et al. (2014) observing an acute increase in $\mathrm{T}$, despite previous literature into vascular occlusion failing to observe a $\mathrm{T}$ response (Reeves et al. 2006; Madarame et al. 2008; McCall et al. 1999). It was proposed that the more intense nature of the exercise prescribed (five sets of five repetitions with 70\% 1RM) along with vascular occlusion stimulus contributed to these results. Interestingly, in the combined group results, the $\Delta \mathrm{T}$ and $\Delta$ blood lactate results both correlated to cuff pressure, therefore, the pressure applied may be speculated to have been the additional stimulus to exercise to increase metabolic accumulation and affected blood lactate. However, as a resistance only condition was not included in the study design, conclusions cannot be drawn whether a similar metabolic response and consequent increase in $\mathrm{T}$ would have resulted without the addition of vascular occlusion.

When examining homogenous groups, in both male and female only groups, $\Delta \mathrm{T}$ pre-to postexercise and $\Delta$ blood lactate was not significantly correlated which may suggest alternative mechanisms other than metabolic response were influencing T. For example, additional reports have suggested hemoconcentration and decreased metabolic clearance as two potential mechanisms to explain exercise associated $\mathrm{T}$ increases (Keizer et al. 1980). As neither mechanisms were measured in the current study, conclusions cannot be drawn and only speculated that either of these mechanisms may have influenced $\mathrm{T}$ response. It would have been beneficial to measure heart rate and blood pressure to determine cardiovascular response to identify if this was having any additional impact. Cardiovascular response is variable within the literature and dependent on training status and mode of exercise. Renzi et al. (2011) identified a greater increase in HR when walking was completed with occlusion in contrast to a control condition. Similar results were also stated for the impact on blood pressure. However, these results were completed with 17 'healthy volunteers'. Whereas Bunevicius et al. (2016) reported a slightly higher HR in the control group, compared to exercise completed at 40\% 1RM with vascular occlusion. 
However, a small increase in systolic blood pressure was observed with vascular occlusion compared to the control. These results were completed within well-trained athletes and concluded low intensity exercise with occlusion did not result in significant overload of cardiac function. It would be beneficial to establish HR and cardiac response to higher intensity (i.e. 70\% 1RM) with vascular occlusion in future research to identify if this would increase cardiovascular stress and subsequent positive or negative effects on performance immediately and $5 \mathrm{~h}$ post exercise.

A novel aspect of the current study was the investigation of morning exercise and hormonal priming in female athletes. A large portion of research into the effect of exercise on T response is completed in males as previously outlined. However, the results are not equivocal in women, with both increases (Nindl et al. 2001; Copeland et al. 2002) and no changes (Linnamo et al. 2005; Consitt et al. 2001; Kraemer et al. 1993) in T response to exercise. For example, Kraemer et al. (1991) measured serum T levels before, during, and after resistance exercise and found that circulating $\mathrm{T}$ is significantly increased in males and is not changed in females throughout the duration of the exercise. However, within the current study, female T response followed a similar reaction to males and combined group results; a decrease of $17.2 \pm 21.1 \%$ was displayed in the control from AM to PM whereas morning exercise attenuated this decline to $1.6 \pm 25.5 \%$ within RE+VO. Similarly, females also showed an acute increase in T response $(8.3 \pm 17.5 \%)$ to exercise with vascular occlusion, which is comparable to the increase seen in male participants (13.6 \pm 26.3\%). The aforementioned studies investigated females that were active, however research completed by Cook et al. (2012) identified baseline measures of $\mathrm{T}$ were significantly higher at weekly time points over 12 weeks in elite (international level athletes) $\left(87 \mathrm{pg} \cdot \mathrm{ml}^{-1}\right)$ than non-elite (national level athletes) $\left(41 \mathrm{pg} \cdot \mathrm{ml}^{-1}\right)$ female athletes. This may support the results of this study as the average baseline for females involved was $97.9 \pm 24.5 \mathrm{pg} \cdot \mathrm{ml}^{-1}$, providing evidence for the international calibre of athletes tested as well as highlighting reasons for comparisons of $\mathrm{T}$ response in females to previous research. Furthermore, only three females were using oral contraceptives, which as previously determined by Crewther et al. (2015), lowers baseline and T response to exercise, potentially influencing previous research and explaining variation in results of the current research. Therefore, the current results suggest the use of morning exercise to attenuate the circadian decline in $\mathrm{T}$ in elite female athletes, however this did not translate into improved afternoon performance, consistent with combined group results.

Cortisol response between conditions varied in females compared to other examined groups. Within the control condition, $C$ declined by $60.9 \pm 17.9 \%$, whereas only a decrease of $30.2 \pm 36.9 \%$ was observed following RE+VO. Afternoon C levels were lower in Control (0.190 \pm 0.08 $\mathrm{ug} \cdot \mathrm{dL}^{-1}$ ) compared to $0.246 \pm 0.15 \mathrm{ug} \cdot \mathrm{dL}^{-1}$ in $\mathrm{RE}+\mathrm{VO}$ consistent with previous research (Cook et al. 2014). Cortisol is the major stress hormone which targets an array of both peripheral and central 
processes (Lupien et al. 2007). The regulation of stress-induced as well as basal C secretion is thus essential for the maintenance of homeostasis (McEwen 2000; Tsigos \& Chrousos 2002). Cortisol is released from the adrenal cortex in response to stress during exercise (Kon et al. 2012) and promotes catabolism via decreased protein synthesis and increased protein degradation (Kraemer \& Ratamess 2005). Contrasting results have reported the influence of C on performance. Sale et al. (2008) provided evidence of the negative effects of increased C on neuromuscular functioning, but this was in the mornings in comparison to afternoon performance. Opposing research by Teo et al. (2011) found acute diurnal variations of C did not display strong relationships with the circadian rhythm of force or power output. Although both hormonal response (C) and physical performance displayed a circadian pattern, no correlation was found between the two variables, therefore, the acute elevation in $\mathrm{C}$ may not negatively impact performance in the current study. Of further interest was the difference in response of females compared to males and combined group results. Previous research has shown no difference in diurnal C patterns between contraceptive users and non-users (Vibarel-Rebot et al. 2015) and furthermore menstrual cycle stage has not influenced stability of C (Liening et al. 2010). Therefore, differences in $\mathrm{C}$ response are likely to be a result of resistance exercise combined with vascular occlusion and females displaying an increased stress response. If performance scientists are applying this strategy with females either for pre-competition or training it should be considered that females may display an elevated $\mathrm{C}$ response which may impact training adaptation or affect subsequent performance.

Despite $\mathrm{RE}+\mathrm{VO}$ reducing the circadian decline in $\mathrm{T}$, across all groups, no PM performance effects were observed for CMJ or IMTP; findings which contradict previous work (Russell et al. 2015; Cook et al. 2014). Results were similar in performance change between all groups, except the improvement in IMTP peak force was greater in weightlifting only athletes $(3.2 \pm 7.7 \%)$. Previous research has suggested baseline strength and power may determine responsive properties for example, a significant effect of baseline strength on the relationship to $\mathrm{T}$ and performance in squat performance has been observed; $T$ correlated to squat performance $(r=0.92)$ in strong participants compared to weaker counterparts $(r=0.35)$ (Crewther et al. 2012). The expression of force and power may be dependent on individual variation in salivary free $\mathrm{T}$ concentration and/or existing strength and power levels (Crewther et al. 2012) which may support that weightlifting athletes have increased baseline strength and power compared to field athletics and thus this group alone showed (although not significant) a greater improvement in PM performance in a test specific to their competition performance. This may also explain the individual variation observed in results, especially of the IMTP in which 13 out of 22 subjects improved peak force ( 2 to $17 \%$ ). 
Alternatively, individual variation in factors associated with the efficacy of RE+VO, such as the balance between muscle activation and fatigue (contractile/metabolic impairment), may have contributed to such findings. Vascular occlusion with resistance exercise implicates greater muscular activation to maintain the same total work output (Takarada et al. 2000; Takarada et al. 2002; Yasuda et al. 2008; Fahs et al. 2015) and additional motor unit activity and enhanced recruitment of type II motor units (Moore et al. 2004) is required to maintain force levels following resistance exercise combined with vascular occlusion (Fatela et al. 2016); even when muscle damage remains unchanged (Wilson et al. 2013). Also to acknowledge, Kluess \& Wood (2005) observed more pronounced vagal withdrawal concomitant with increased sympathetic activity following vascular occlusion. As the recovery of heart rate variability after exercise with vascular occlusion was delayed, this indicates a greater autonomic disruption when blood flow is occluded during exercise (Okuno et al. 2014), consequently impacting PM performance and highlighting the importance of balance between muscle activation and fatigue. Therefore, further research is required to identify individual factors that contribute to the optimisation of resistance exercise with vascular occlusion for use prior to subsequent exercise whether implemented immediately or $5 \mathrm{~h}$ before.

Several methodological inconsistencies have generated conflicting findings between studies. For example, the vast majority of studies have implemented an occlusion protocol prescribing an arbitrary restrictive pressure for all participants or based on systolic blood pressure at rest (Fatela et al. 2016). Recent findings however indicate that neither one of these previously used approaches represents an effective strategy for controlling the magnitude of blood flow restriction in the lower body (Loenneke et al. 2012). If restrictive pressures are too low, muscular responses may not be significantly augmented (Scott et al. 2015). Furthermore, extremely high pressures (i.e. those that occlude arterial inflow during inter-set rest and/or exercise) may not enhance muscular development more than moderate pressures (Scott et al. 2015). It is accepted that vascular occlusion pressure should be high enough to occlude venous return from the muscles, yet low enough to maintain arterial inflow into the muscle (Loenneke et al. 2014). Logic therefore dictates that vascular occlusion should not be universally applied at an absolute pressure, but should vary relative to each individual (Loenneke et al. 2013).

In both males and females, previous research has suggested thigh girth and arterial blood pressure are important determinants for vascular occlusion (Loenneke et al. 2015), offering a practical approach to individualising cuff pressure, consequently the present study used these two determinants to individualise cuff pressure. This was an important step, considering required individualisation of strategies for elite athletes and also considering Hunt et al. (2016) highlighted $60 \%$ reduction in popliteal blood flow was achieved at lower external pressures in females 
compared to males. Reports suggest this is likely to be due to differences between sexes in local vascular function (Hunt et al. 2016). Contrasting previous research, Hunt et al. (2016) also highlighted mean arterial pressures and adipose thickness were the largest independent determinants of lower partial occlusion pressures. Less is understood about the impact of tissue type (muscle or fat) on the level of vascular occlusion, but it is likely the degree of intramuscular pressure depends on the architecture features of the muscle (Hunt et al. 2016). Previous research has investigated protocols in non-athletic groups, therefore the impact of tissue type and other determining factors may vary the effectiveness of the protocol for elite athletes. Extended research is required to determine the efficacy of best practice in elite athletes to optimise cuff pressure if ultrasound Doppler is not viable.

Despite providing a novel insight into the use of AM resistance exercise combined with vascular occlusion on PM performance in elite athletes, several experimental limitations are acknowledged. The study design limits conclusions which can be drawn from the results; a traditional experimental study design of control versus one manipulation was completed (similar to design used by Cook et al. 2012), however this inherently prevents identifying if the combined effect of resistance exercise and vascular occlusion attenuated the decline in T. Only from the use of previous research recommending the requirement for volume/intensity threshold to be achieved for an increase in $\mathrm{T}$ response and previous morning exercise results can speculations be made; however, it cannot be inferred if occlusion was responsible for the $\mathrm{T}$ response. The addition of a non-occluded AM resistance exercise-only trial would have allowed further delineation of the effects of AM resistance exercise with vascular occlusion on physiological and performance responses. However, the recruitment of the international standard of participants to the study design, while a strength of the protocol, inherently posed significant time-pressures which precluded the addition of a third experimental condition. Additionally, hypertrophied muscles have a greater thickness and volume and subsequently vascular occlusion may occur at relatively lower pressures when compared to applying pressure to adipose tissue (Hunt et al. 2016). Despite our attempts to individualise the occlusion pressures implemented in this study (Loenneke et al. 2015), there may have been between-participant variation in the degree of occlusion experienced during vascular occlusion. Further research into individual factors modulating the degree of pressure experienced by participants in vascular occlusion will likely better inform the use of this ergogenic modality in an athletic environment.

\subsection{PRACTICAL IMPLICATIONS}

This study has shown that resistance exercise combined with vascular occlusion can reduce the decline in T from AM to PM. However, this data did not improve PM performance in all athletes. For practical application, resistance exercise combined with vascular occlusion can be used by 
performance scientists, coaches and athletes to reduce the decline in T from AM to PM that is caused by the circadian decline, by completing resistance exercise at 70\% $1 \mathrm{RM}$ with vascular occlusion. Due to the variability of individual responses, this should be trialled with each athlete, consequently, the response will be directly applicable to the individual athlete and can be implemented into a pre-competition routine for those individuals which show a positive performance response in the afternoon. It should also be practiced, ensuring tolerance of resistance exercise with vascular occlusion, this may result in enhanced performance once the individual has increased familiarity and use of vascular occlusion.

\subsection{CONCLUSION}

To conclude, the current study identifies a reduced decline in $\mathrm{T}$ over $5 \mathrm{~h}$ when resistance exercise combined with vascular occlusion was completed in the morning. This is believed to be a result of an increased metabolic response which caused a consequential increase in $\mathrm{T}$ concentration after exercise. However, a reduced decline in T concentration at the time of PM performance measures did not result in an improved performance, with results showing variability in individual performance. This study offers novel findings into the use of resistance exercise with vascular occlusion for AM hormonal priming for male and females, but further research is required prior to practical implementation. 


\section{Chapter 5 - The effect of ischemic preconditioning on maximal swimming performance}

\subsection{STUDY INFORMATION}

Information presented in the previous chapter was specific for sports involving strength and power, and therefore investigated an appropriate pre-competition strategy specific for the nature of these sports. Other sports contrast in underpinning mechanisms which influence performance and therefore the previously discussed pre-competition strategy may not be appropriate to impact performance. Aerobic/anaerobic energy requirements are important for swimming performance and therefore pre-competition strategies specific to these mechanisms are of primary importance. Consistent with the previous chapter, the following chapter aimed to explore the use of limb IPC as a pre-competition strategy but adjusting the protocol to apply the strategy during passive rest and focus on mechanisms specific to improve swimming performance.

Aim: Investigate the use of limb IPC applied in the early ( $2 \mathrm{~h})$ and late phase (24 h) on swimming TT performance

\section{Objectives:}

- To identify whether the effect of IPC on swimming performance is time dependent

- To identify if improvement in swimming performance is achieved following limb IPC implemented $24 \mathrm{~h}$ prior to performance to harness the mechanisms associated with the late phase of IPC

- To identify if limb IPC at $2 \mathrm{~h}$ or $24 \mathrm{~h}$ influences physiological mechanisms associated with blood lactate and gases

Hypothesis: limb IPC will improve swimming TT performance at both $2 \mathrm{~h}$ and $24 \mathrm{~h}$ in comparison to a control condition

Outcome measures: blood lactate and gas measurements, RPE and swimming TT 


\subsection{INTRODUCTION}

During international swimming events athletes are required to perform two to three maximal efforts following months or even years of training and preparation, with marginal differences of $<0.5 \%$ separating medal and non-medal positions (e.g. difference between sixth and third place in the men's and women's 100 m at World Championships; FINA, World Championship results 2017 - http://www.fina.org/event/17th-fina-world-championships/results). In addition to the benefits of training, previous research has shown the importance of competition warm-up intensity (Neiva et al. 2014), timing of warm-up (West et al. 2013b) and use of active heating and land-based activation exercises (McGowan, Pyne, et al. 2016; McGowan, Thompson, et al. 2016) as competition-day strategies to improve subsequent swimming performance. Limb IPC has also been reported to improve indices of athletic performance when used between $15 \mathrm{~min}$ and $8 \mathrm{~h}$ before performance assessments (Horiuchi 2017).

The benefits of limb IPC to improve athletic performance have been previously observed in time to exhaustion (e.g. De Groot et al. 2010), anaerobic specific performance tests (e.g. Jean-St-Michel et al. 2011) and repeated sprint ability (e.g. Patterson et al. 2015) (Refer to Section 2.5.3). It has been reported that limb IPC induces acute vascular adaptations, resulting in local vasodilation and enhanced blood flow (Tapuria et al. 2008). Consequently, enhanced functional sympatholysis may speed and increase $\mathrm{O}_{2}$ extraction by means of matching demand with supply (Horiuchi et al. 2015), facilitating an increased aerobic contribution during subsequent exercise. Reports suggest that limb IPC can cause a faster uptake of acetyl-CoA by mitochondria thus maintaining lactate accumulation at a metabolically acceptable level due to greater contribution of aerobically generated ATP for exercise (Jean-St-Michel et al. 2011). Recruitment of higher order motor units via enhanced central motor efferent command also results from limb IPC (Crisafulli et al. 2011), allowing for exercise to be completed beyond the individual's critical threshold by increasing or maintaining the rate of force development and improving subsequent performance.

However, only one study (Seeger et al. 2017) relating to sports performance has differentiated between the observed early and late phase of limb IPC reported within the clinical literature, implementing limb IPC $24 \mathrm{~h}$ prior to a $5 \mathrm{~km}$ running TT. Research suggests that there are two phases resulting from limb IPC; the early phase which begins soon after reperfusion and lasts 3-4 $\mathrm{h}$, whereas the late phase starts 12-24 h after limb IPC (Kuzuya et al. 1993) and last 48-72 h (Pell et al. 1998; Singh \& Chopra 2004). The release of endogenous substances is thought to stimulate post-translational modifications in proteins within the early phase, whereas in the late phase this leads to synthesis of new proteins and altered gene expression (Tapuria et al. 2008). Furthermore, upper limb IPC confers an improvement in endothelial function in the contralateral arm 24-48 $\mathrm{h}$ 
later (Hausenloy \& Yellon 2008). Accordingly, owing to the timing of pre-competition practices and regulations in athletic competitions (e.g., the use of pre-competition call-rooms within $20 \mathrm{~min}$ of competition starting), the late phase of limb IPC may offer another practical option, to coincide with competition timings to further optimise swimming performance on the day of competition.

With a specific emphasis on swimming performance, limb IPC may be beneficial for 100 to 400 $\mathrm{m}$ swimming performance due to the resultant increase in contribution of ATP generated from the aerobic system (Rodriguez \& Mader 2003). To date, three studies (Jean-St-Michel et al. 2011; Lisbôa et al. 2017; Marocolo et al. 2015) have identified a positive effect of implementing limb IPC prior to swimming performance. For example, Jean-St-Michel et al. (2011) reported that five min of ischemia followed by five min of reperfusion, repeated for four cycles, implemented 45 min prior to $100 \mathrm{~m}$ swimming TT improved personal best swimming times by $1.1 \%$. Most recently, Lisbôa et al. (2017) applied limb IPC $1 \mathrm{~h}, 2 \mathrm{~h}$ and $8 \mathrm{~h}$ preceding a $50 \mathrm{~m}$ TT performance, with performance improvements of $1.0 \%$ and $1.2 \%$ in $2 \mathrm{~h}$ and $8 \mathrm{~h}$ conditions, respectively. The previous research relating to limb IPC and swimming performance has investigated the effects of the early phase of limb IPC on performance as application has been $<12 \mathrm{~h}$ prior to performance. However, for short duration events (i.e. 10-90 s), a recent meta-analysis showed that a longer duration between limb IPC and exercise resulted in a higher effect size; suggesting that limb IPC may be dependent on the timing of the preconditioning strategy relative to the start of subsequent performance (Salvador et al. 2016). Research is yet to investigate if the delayed phase of limb IPC can enhance swimming performance when applied at least $12 \mathrm{~h}$ prior to competition, a strategy which may be attractive for coaches and swimmers. Consequently, the purpose of this study was to investigate the impact of limb IPC on swimming TT performance $2 \mathrm{~h}$ (early phase) and $24 \mathrm{~h}$ (late phase) after eliciting limb IPC in competitive swimmers.

\subsection{METHOD}

\subsubsection{Experimental overview}

Twenty National and International-level swimmers participated in a randomised, crossover design that involved three sessions (Con, IPC-2h, IPC-24h) separated by seven days. Timing of limb IPC completed in conditions were implemented in line with previous research complete by Seeger et al. (2017) and Lisbôa et al. (2017). Occlusion cuffs were applied bi-laterally at the most proximal point of each thigh and intermittently inflated to an individualised cuff pressure determined from thigh girth and resting blood pressure for a total of $40 \mathrm{~min}$ in IPC-2h and IPC-24h. In Con, cuffs were applied for the same duration (total $40 \mathrm{~min}$ ), however cuff pressure was inflated to $15 \mathrm{mmHg}$. A self-selected (100 or $200 \mathrm{~m}$ ) swimming TT (assessing total time, $50 \mathrm{~m}$ split times, stroke count; 
SC, and stroke rate; SR, time underwater off starts and turns) followed intervention administration and physiological markers ( $\mathrm{pH}$, blood gases and lactate concentrations) assessed at pre-limb IPC, post-limb IPC, pre-TT and post-TT.

\subsubsection{Participants}

Following ethical approval, 18 (6 females, 12 males) National-and International-level swimmers (age; $20 \pm 2 \mathrm{y}$, mass; $71.1 \pm 9.6 \mathrm{~kg}$, stature; $178.4 \pm 9.6 \mathrm{~cm}$, Training experience; $9.6 \pm 2.7 \mathrm{y}$ ) participated in the study. All participants had qualified for and competed at British swimming National competitions. Participants were informed of the experimental procedures, the purpose and possible risks associated with the study, and provided written informed consent before participation.

\subsubsection{Main trial procedures}

After familiarisation, participants were required to attend the testing venue on three occasions (Con, IPC-2h, IPC-24h) in a randomised order. Main trials were performed in an enclosed $50 \mathrm{~m}$ swimming pool within the participants normal training environment. To minimise the effects of biological rhythms, the timing of measurements was consistent between trials. To control for varying levels of weekly fatigue, testing was conducted on the same day of the week in a stable, maintenance phase of training. Participants were required to refrain from alcohol and intense physical exercise in the $24 \mathrm{~h}$ preceding trials and between limb IPC and swimming TT performance.

On arrival for main trials, participants were required to rest for ten min to allow for resting blood pressure to be recorded (Omron Healthcare, Europe; systolic $>140 \mathrm{mmHg}$ and/or diastolic $>90$ $\mathrm{mmHg}$ precluded further study involvement). Once blood pressure was recorded, thigh girth was measured for determination of cuff pressure and a capillary blood sample was taken. Occlusion cuffs, as detailed in section 3.4, were then applied to the most proximal point of the thighs, with participants assuming a supine position. Cuffs were manually inflated to either $15 \mathrm{mmHg}$ (Con) or an individualised cuff pressure as determined in section 3.4 (IPC-2h, IPC-24h), for a total of 40 min consisting of four cycles of five min occlusion and five min reperfusion. The individualised cuff pressures ranged from 160 to $228 \mathrm{mmHg}$, whereas cuff pressure was $15 \mathrm{mmHg}$ in the Con condition 


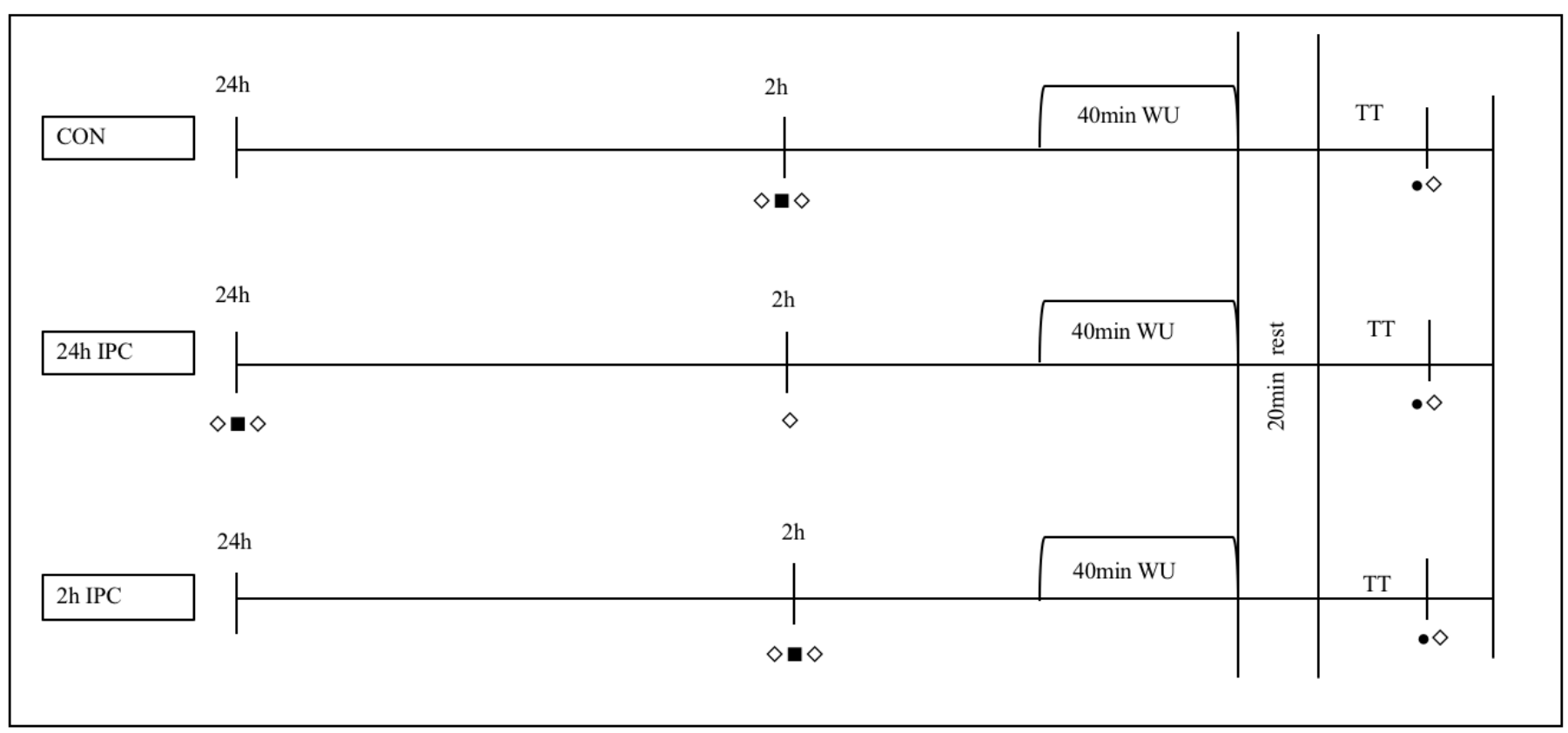

Figure 5.1: Overview of timeline of data collection. IPC - ischemic preconditioning, WU - warm up, TT - time trial, $(\diamond)$ blood sample, ( ischemic preconditioning, $(\bullet)$ RPE 
Following the completion of the limb IPC protocol, participants rested accordingly for $24 \mathrm{~h}$ or 2 $\mathrm{h}$; intense physical activity was restricted during the $24 \mathrm{~h}$ and all participants arrived at the swimming pool and rested for $3 \mathrm{~h}$ prior to TT regardless of the condition, cuffs were applied during this period for IPC-2h and Con. A standardised race swimming warm up (40-min; Appendix E) was performed $1 \mathrm{~h}$ prior to a swimming TT as prescribed by the coaches and followed the structure of individuals race warm up, variance existed in warm up regarding stroke which was specific to the stroke being used to complete the swimming TT. A 20-min post-warm-up rest period at the swimming pool replicated pre-competition call room requirements. This was immediately followed by a maximal swimming TT (100 m: $n=15,200 \mathrm{~m}: n=5)$, completed on the participants' chosen stroke, in accordance with FINA rules. Participants completed the TT individually, starting from a block and taking off after an audible starting signal. During study design it was discussed whether time trials were to be completed individually or in a simulated competition setting. However, due to randomisation of the conditions, a competition format may have resulted in differences in performance due to racing opponents rather than due to the effects of interventions, making it hard to draw conclusions of the effect of limb IPC on performance. Rating of perceived exertion was recorded using the Borg (1982) scale (Appendix F) on completion of the race. From the TT, SR, SC, $50 \mathrm{~m}$ split times, time underwater off the start and turns and total time were calculated retrospectively from video recordings. Equation 1 was used to determine SR; for each $25 \mathrm{~m}$ of the TT SR was calculated, the mean $\pm \mathrm{SD}$ was then calculated for each $50 \mathrm{~m}$. To ensure acceptable reliability of the SR measurement, intra-observer tests were completed. The analyst viewed two randomly selected TT performances ten times over a two-week period under the same conditions. The CV was calculated to identify the measurement error; this resulted in a low, acceptable percentage of error $(\mathrm{CV}=0.2 \%)$.

Equation 4.1: Stroke rate $=\underline{(\text { Number of complete strokes over } 25 \mathrm{~m} \mathrm{x} \mathrm{60)}}$

(Time of hand entry 1 - time of hand entry 2 )

Where hand entry 1 is the first-hand entry at the start of $25 \mathrm{~m}$ and hand entry 2 is the hand entry at the end of $25 \mathrm{~m}$, recorded in seconds.

A capillary blood sample was taken pre-limb IPC, post-limb IPC, pre-TT and post-TT to measure $\mathrm{pH}$ and blood gases as specified in section 3.6.2. The measurements were taken immediately pre and post; post measurements were all completed within $2 \mathrm{~min}$ of end of IPC and also after finishing swimming TT performance. This was analysed using a portable analyser (ISTAT 1; 300G) and associated cartridges (CG4+; Abbott, point of care testing, Arbroath, UK) and calibrated as detailed in section 3.6.2. Blood gases and $\mathrm{pH}$ were analysed using these methods which have previously been compared (Verwaerde et al. 2002) against two auto-calibrated analysers ( $r>0.993$ ) (section 3.6.2). 


\subsubsection{Statistical analysis}

All data is presented as mean $\pm \mathrm{SD}$. Following confirmation of parametric assumptions, repeated measures MANOVA with Bonferroni adjustment assessed between-trial differences for variables with multiple time points per trial (i.e. blood lactate, $\mathrm{pH}, \mathrm{sO}_{2} \%, \mathrm{PO}_{2}, \mathrm{PCO}_{2}, \mathrm{HCO}_{3}$ and $\mathrm{BEecf}$ ). One-way ANOVA assessed between-trial differences for all performance variables from the swimming TT and RPE recorded post-TT. Smallest worthwhile change was calculated according to Hopkins et al. (1999) in which 0.3 of the coefficient of variation (CV) gives the smallest important change in performance and increase chances of a medal. 0.3 of the coefficient was calculated for Control/IPC-2h and Control/IPC-24h and was compared to the change in performance between the control and both conditions. If the change in performance was greater than the smallest important change calculated, it was deemed to have a smallest worthwhile change. Please refer to Chapter 3, section 3.9 for more detail.

\subsection{RESULTS}

\subsubsection{Physiological responses}

In the whole group, exercise significantly affected blood parameters; following swimming TT, $\mathrm{pH}$ decreased by $0.22 \pm 0.08(2.9 \pm 1.1 \%)$ in all conditions $\left(\mathrm{p}<0.001 ; \eta^{2}=0.866\right)$ and blood lactate increased pre-to post-TT $\left(\mathrm{p}<0.001 ; \eta^{2}=0.923\right)$ by $12.87 \pm 3.62 \mathrm{mmol} \cdot \mathrm{L}^{-1}, 12.41 \pm 4.02 \mathrm{mmol} \cdot \mathrm{L}^{-1}$ and $13.27 \pm 3.81 \mathrm{mmol} \cdot \mathrm{L}^{-1}$ in IPC-2h, Con and IPC-24h, respectively (88-89\%) (Figure 5.2). Base excess, $\mathrm{HCO}_{3}$ (Figure 5.2), $\mathrm{TCO}_{2}$ (IPC-2h: $-12.90 \pm 3.92 \mathrm{mmol} \cdot \mathrm{L}^{-1}$; Con: $-11.55 \pm 7.61 \mathrm{mmol} \cdot \mathrm{L}^{-1}$; IPC-24h: $9.90 \pm 8.40 \mathrm{mmol} \cdot \mathrm{L}^{-1} ; \mathrm{p}<0.001 ; \eta^{2}=0.939$ ) and $\mathrm{sO}_{2} \%$ (IPC-2h: $-0.16 \pm 1.86 \%$; Con: $+0.20 \pm 1.93 \%$; IPC-24h: $+0.47 \pm 2.10 \%$; $<0.001 ; \eta^{2}=0.130$ ) were significantly different preTT to post-TT (Table 5.1). However, there were no differences between trials in any of the blood parameters $(\mathrm{p}>0.05)$.

When examining results split into $100 \mathrm{~m}$ and $200 \mathrm{~m}$ disciplines physiological responses showed the same responses as the group data (Table 5.1), blood parameters displayed a time response from swimming TT $(\mathrm{p}<0.001)$ but were not affected by condition (Figure 5.3).

\subsubsection{Performance responses}

Trial did not affect performance for $100 \mathrm{~m}$ (p=0.995; IPC-2h: $64.94 \pm 8.33 \mathrm{~s}$; IPC-24h: $64.67 \pm$ $8.50 \mathrm{~s}$; Con: $64.94 \pm 8.24 \mathrm{~s}$ ), $200 \mathrm{~m}$ (p=0.405; IPC-2h: $127.70 \pm 10.66 \mathrm{~s}$; IPC-24h: $129.26 \pm 12.99$ s; Con: $130.19 \pm 10.27$ ) or combined total time (IPC-2h: $84.27 \pm 31.52 \mathrm{~s}$; IPC-24h: $79.87 \pm 29.72$ $\mathrm{s}$; Con: $80.55 \pm 31.35 \mathrm{~s}$ ). Combined group differences from Con to IPC-2h was $0.3 \pm 2.2 \%$ and $0.2 \pm 1.4 \%$ for the difference in performance between Con and IPC-24h. When divided into 
distances there was no difference between Con and IPC-2h performance in $100 \mathrm{~m}(0.0 \pm 1.2 \%)$ or between Con and IPC-24h $(0.1 \pm 1.1 \%)$. Performance of $200 \mathrm{~m}$ TT was negatively affected by both IPC-2h $(-0.5 \pm 1.3 \%)$ and IPC-24h $(-1.5 \pm 2.0 \%)$.

Variation in individual performance was observed as displayed in Figure 5.4; the individual performance differences displayed 9 participants had a smallest worthwhile change in performance following the use of IPC-2h (Table 5.3), with variation in change required ranging from 0.00 $0.29 \%$. Whereas 11 participants showed a smallest important change after completion of IPC-24h (Table 5.4) with a required change ranging from $0.01-0.45 \%$.

No significant effects between conditions for any of the performance variables were observed when analysed as a group, displayed in Table 5.2; being, split time for the first $50 \mathrm{~m}(\mathrm{p}=0.968)$, start time $(\mathrm{p}=0.817)$ and turn time at $50 \mathrm{~m}(\mathrm{p}=0.924)$ for instance. RPE also did not vary between conditions ( $p=0.723$ ) or when exploring specifically for $100 \mathrm{~m}(\mathrm{p}=0.408)$ and $200 \mathrm{~m} \mathrm{TT}(\mathrm{p}=0.260)$. 
Table 5.1: Physiological responses across data collection time points, displayed for three conditions tested

\begin{tabular}{|c|c|c|c|c|c|c|c|c|c|}
\hline IPC-2h & & pH & $\begin{array}{l}\text { Base excess } \\
\left(\mathrm{mmol.}^{-1}\right)\end{array}$ & $\begin{array}{l}\mathrm{HCO}_{3} \\
\left(\mathrm{mmol.l}^{-1}\right)\end{array}$ & $\begin{array}{l}\mathrm{TCO}_{2} \\
\left(\mathrm{mmol}_{\left.. \mathrm{I}^{-1}\right)}\right.\end{array}$ & $\mathrm{SO}_{2}(\%)$ & $\begin{array}{l}\text { Blood La } \\
\left(\mathrm{mmol}^{-\mathrm{l}^{-1}}\right)\end{array}$ & RPE & HR (bpm) \\
\hline \multirow[t]{2}{*}{ Pre-occlusion } & Average & 7.41 & 0.05 & 24.79 & 25.95 & 94.47 & 1.45 & & \\
\hline & SD & 0.01 & 1.31 & 1.38 & 1.39 & 1.61 & 0.46 & & \\
\hline \multirow[t]{2}{*}{ Post occlusion } & Average & 7.42 & 0.32 & 23.57 & 25.80 & 94.65 & 1.55 & & \\
\hline & SD & 0.02 & 1.67 & 4.95 & 1.47 & 1.35 & 0.39 & & \\
\hline \multirow[t]{2}{*}{ Pre TT } & Average & 7.42 & 0.32 & 23.57 & 25.80 & 94.65 & 1.55 & & \\
\hline & SD & 0.02 & 1.67 & 4.95 & 1.47 & 1.35 & 0.39 & & \\
\hline \multirow[t]{2}{*}{ Post TT } & Average & 7.20 & -13.79 & 12.54 & 13.58 & 90.53 & 13.44 & 17.5 & 184.43 \\
\hline & SD & 0.07 & 8.23 & 2.81 & 2.93 & 20.74 & 3.08 & 0.86 & 8.27 \\
\hline \multicolumn{10}{|l|}{ Control } \\
\hline \multirow[t]{2}{*}{ Pre-occlusion } & Average & 7.41 & -0.13 & 23.43 & 24.63 & 94.38 & 1.38 & & \\
\hline & SD & 0.02 & 1.54 & 5.09 & 5.62 & 1.71 & 0.44 & & \\
\hline \multirow[t]{2}{*}{ Post occlusion } & Average & 7.41 & 0.33 & 25.07 & 25.22 & 94.24 & 1.43 & & \\
\hline & $\mathrm{SD}$ & 0.02 & 2.03 & 1.61 & 5.12 & 1.52 & 0.34 & & \\
\hline \multirow[t]{2}{*}{ Pre TT } & Average & 7.41 & 0.33 & 25.07 & 25.22 & 94.24 & 1.43 & & \\
\hline & $\mathrm{SD}$ & 0.02 & 2.03 & 1.61 & 5.12 & 1.52 & 0.34 & & \\
\hline \multirow[t]{2}{*}{ Post TT } & Average & 7.19 & -15.35 & 12.88 & 13.12 & 94.94 & 12.52 & 17.3 & 186.46 \\
\hline & SD & 0.08 & 4.80 & 3.72 & 5.02 & 1.43 & 3.18 & 1.14 & 8.13 \\
\hline
\end{tabular}


Table 5.1 Continued

\begin{tabular}{|c|c|c|c|c|c|c|c|c|c|}
\hline IPC-24h & & & & & & & & & \\
\hline \multirow[t]{2}{*}{ Pre-occlusion } & Average & 7.40 & -1.47 & 23.39 & 24.53 & 94.00 & 1.10 & & \\
\hline & $\mathrm{SD}$ & 0.02 & 1.37 & 1.27 & 1.33 & 2.15 & 0.63 & & \\
\hline \multirow[t]{2}{*}{ Post occlusion } & Average & 7.40 & -0.94 & 23.69 & 25.00 & 93.17 & 1.10 & & \\
\hline & $\mathrm{SD}$ & 0.03 & 1.35 & 1.20 & 1.28 & 2.09 & 0.72 & & \\
\hline \multirow[t]{2}{*}{ Pre TT } & Average & 7.41 & 0.00 & 23.15 & 25.81 & 89.06 & 1.55 & & \\
\hline & SD & 0.02 & 1.55 & 5.55 & 1.42 & 22.20 & 0.57 & & \\
\hline \multirow[t]{2}{*}{ Post TT } & Average & 7.18 & -16.53 & 11.82 & 12.65 & 95.18 & 13.39 & 17.3 & 183.17 \\
\hline & SD & 0.10 & 5.35 & 3.71 & 3.69 & 1.01 & 3.12 & 0.95 & 9.16 \\
\hline
\end{tabular}

IPC - ischemic preconditioning; TT - time trial; $\mathrm{HCO}_{3}$ - bicarbonate; $\mathrm{TCO}_{2}$ - total carbon dixoxide; $\mathrm{sO}_{2} \%$ - percentage of oxygen saturation; RPE - rate of perceived exertion; HR - heart rate. 
(A)

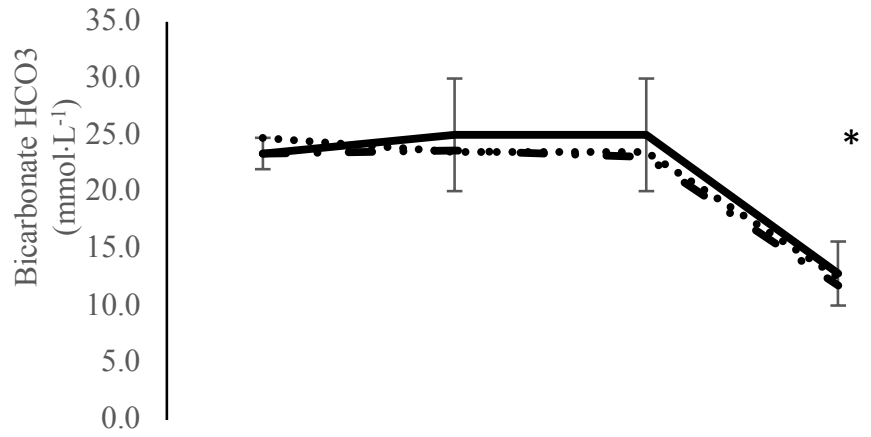

(B)

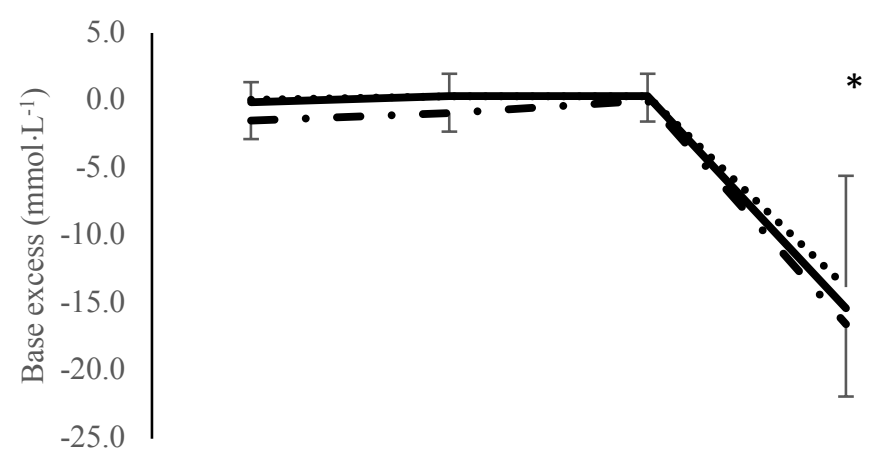

(C)

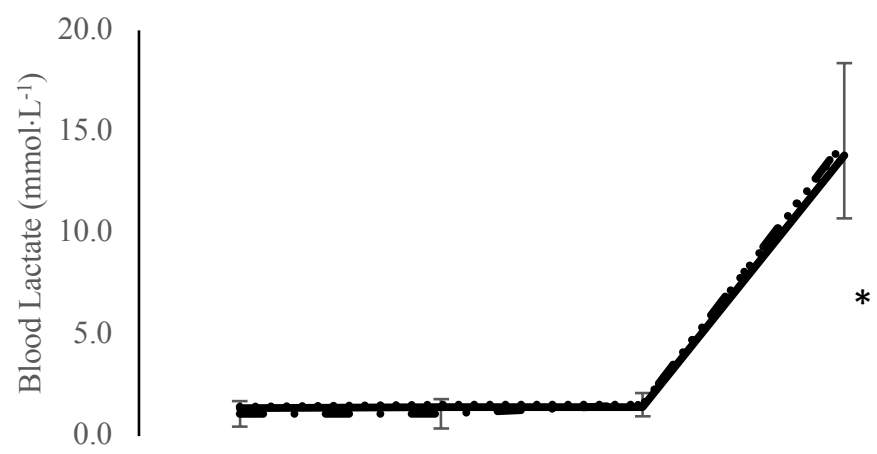

(D)

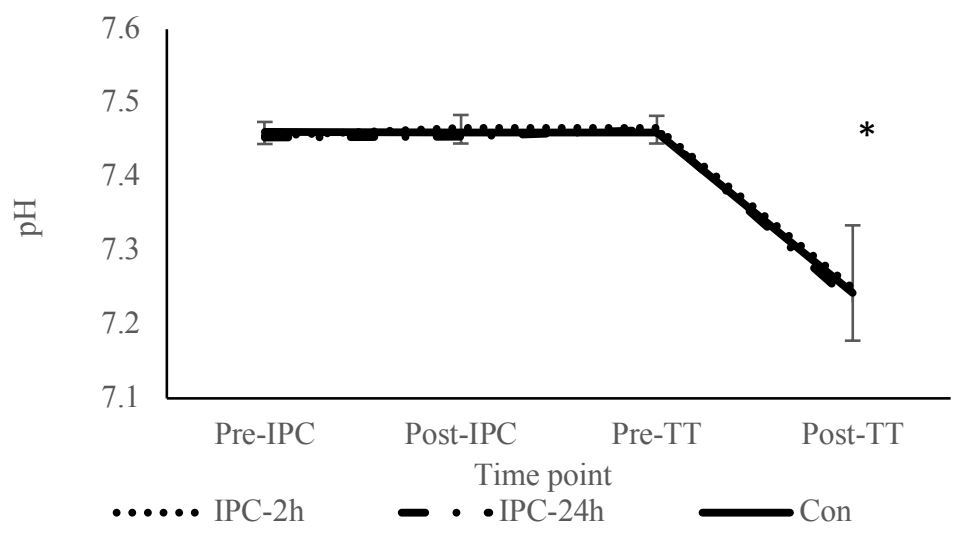

Figure 5.2: Change in blood markers from pre-ischemic preconditioning (IPC) to post-IPC and Pre-time trial (TT) to post-TT. (A) Bicarbonate, (B) Base excess, (C) Blood lactate, (D) pH. * indicates significance difference at sampling time point $(\mathrm{p}<0.05)$ 
(A)
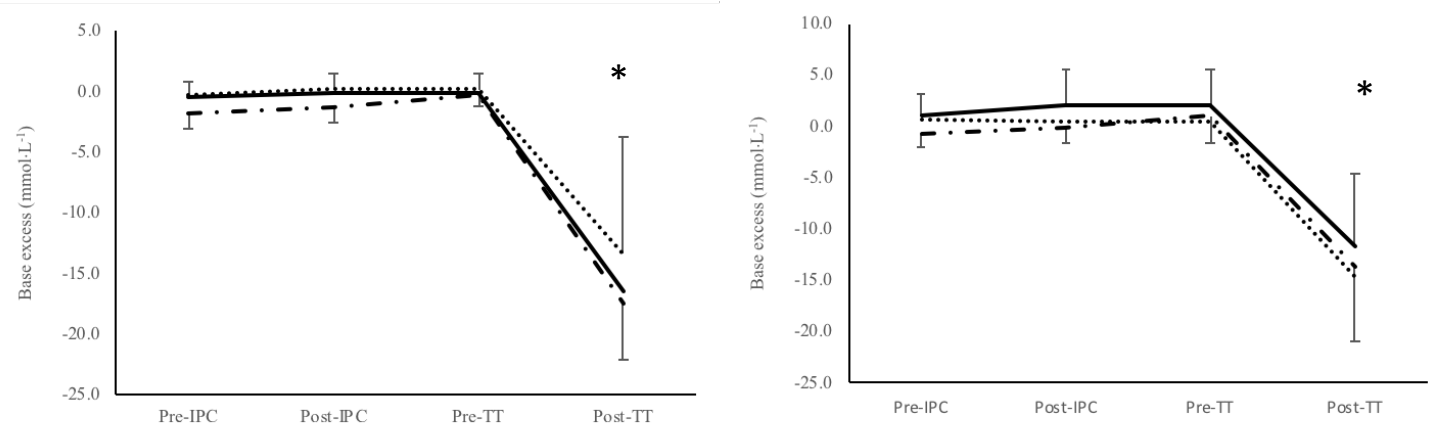

(B)
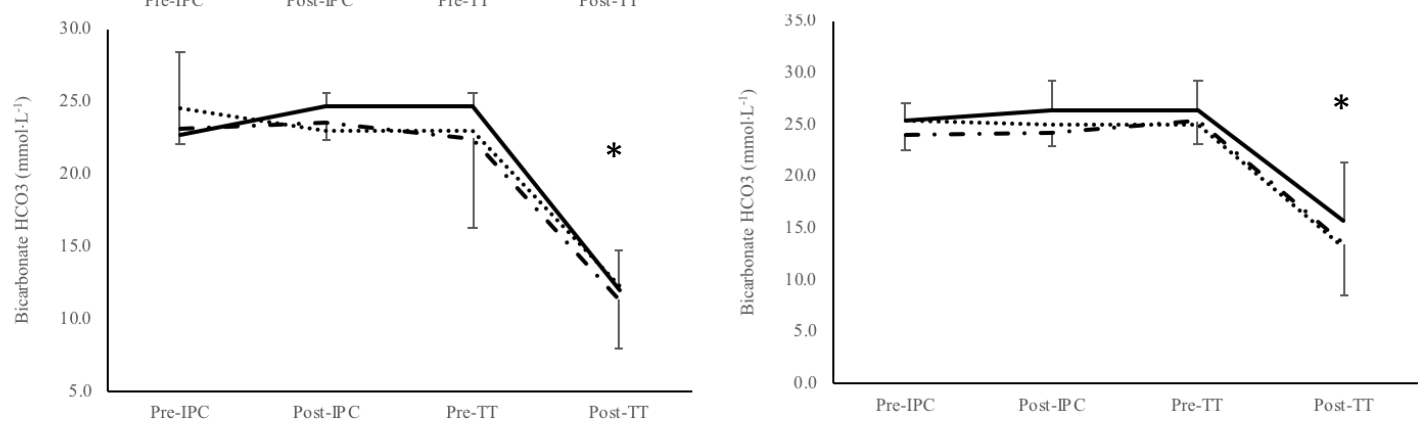

(C)
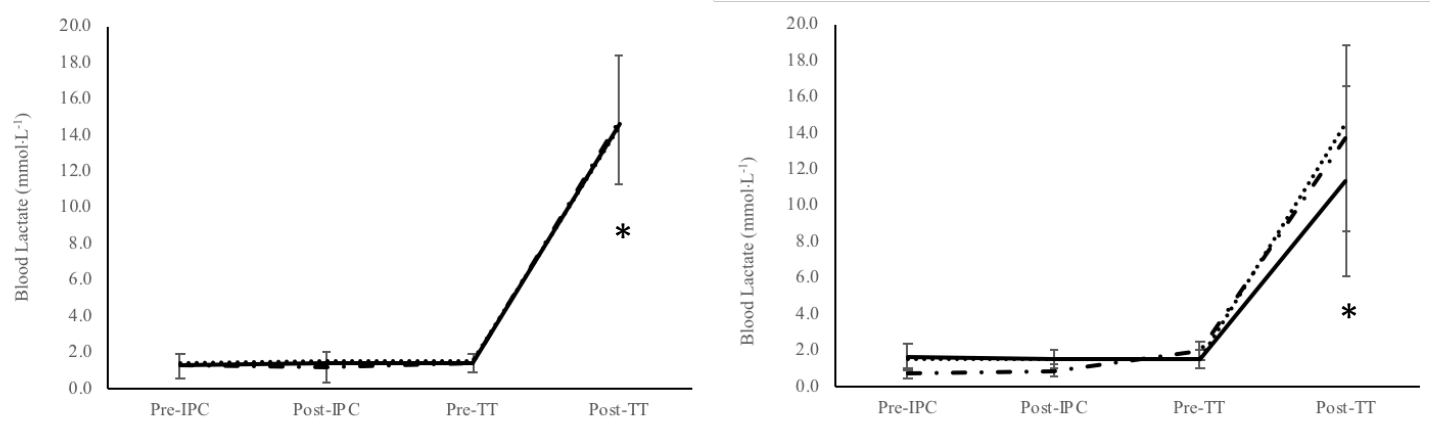

(D)
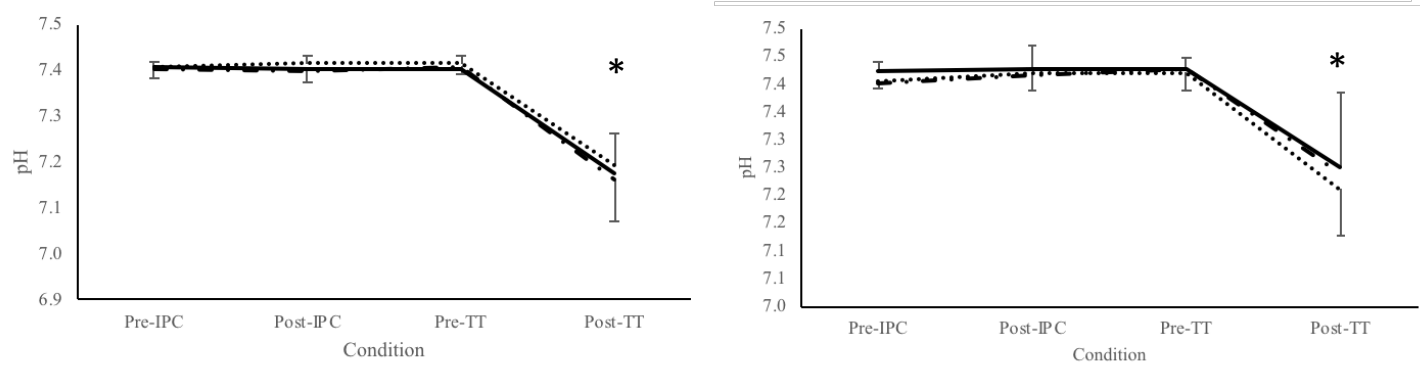

….... $\mathrm{IPC}-2 \mathrm{~h}-\mathrm{Con}-\cdots \mathrm{IPC}-24 \mathrm{~h}$

Figure 5.3: Change in blood markers from pre-ischemic preconditioning (IPC) to post-IPC and Pre-time trial (TT) to post-TT for $100 \mathrm{~m}$ (right) and 200m (left). (A) Bicarbonate, (B) Base excess, (C) Blood lactate, (D) $\mathrm{pH}$. * indicates significance difference at sampling time point $(\mathrm{p}<0.05)$ 
Table 5.2: Performance variables from the swimming time trial $(100 \mathrm{~m}, 200 \mathrm{~m}$ and combined) for the three conditions

\begin{tabular}{llllllll}
\hline Condition & Participants & SC 50 & SC 100 & SR 50 (SPM) & SR 100 (SPM) & Start (s) & Turn 50 (s) \\
\hline Con & Group & $19.3 \pm 2.4$ & $22.2 \pm 3.2$ & $45.3 \pm 8.0$ & $42.5 \pm 7.1$ & $4.9 \pm 1.4$ & $4.2 \pm 1.6$ \\
& $100 \mathrm{~m}$ & $19.4 \pm 2.6$ & $22.7 \pm 3.2$ & $44.6 \pm 8.8$ & $43.1 \pm 7.5$ & $5.0 \pm 1.3$ & $4.3 \pm 1.6$ \\
& 200m & $19.7 \pm 1.5$ & $20.5 \pm 2.7$ & $47.9 \pm 4.1$ & $40.5 \pm 5.5$ & $4.2 \pm 1.8$ & $3.9 \pm 2.1$ \\
IPC-2h & Group & $18.8 \pm 2.6$ & $21.3 \pm 3.2$ & $43.9 \pm 8.1$ & $42.3 \pm 7.4$ & $4.9 \pm 1.4$ & $4.2 \pm 1.7$ \\
& $100 \mathrm{~m}$ & $18.9 \pm 2.7$ & $22.1 \pm 3.1$ & $43.6 \pm 8.9$ & $43.0 \pm 8.4$ & $5.1 \pm 1.4$ & $4.4 \pm 1.6$ \\
& 200m & $18.0 \pm 2.8$ & $19.4 \pm 3.2$ & $43.4 \pm 5.9$ & $40.7 \pm 5.4$ & $4.2 \pm 1.5$ & $3.6 \pm 2.0$ \\
IPC-24h & Group & $18.4 \pm 2.6$ & $21.1 \pm 3.7$ & $43.4 \pm 8.7$ & $42.1 \pm 6.3$ & $5.1 \pm 1.1$ & $4.4 \pm 1.7$ \\
& $100 \mathrm{~m}$ & $18.9 \pm 2.3$ & $22.3 \pm 3.2$ & $43.6 \pm 9.1$ & $44.0 \pm 6.0$ & $5.0 \pm 1.2$ & $4.3 \pm 1.7$ \\
& 200m & $16.8 \pm 3.3$ & $17.0 \pm 1.4$ & $42.6 \pm 8.3$ & $36.6 \pm 2.8$ & $5.4 \pm 1.4$ & $4.9 \pm 1.9$ \\
\hline
\end{tabular}

*SC50 - stroke count for the first $50 \mathrm{~m}$; SC 100 - stroke count for the second $50 \mathrm{~m}$; SR 50 - stroke rate for first $50 \mathrm{~m}$; SR 100 - stroke rate for second $50 \mathrm{~m}$; start - time from dive start to first stroke; Turn 50 - turn time at $50 \mathrm{~m}$. Ninety-five-percent confidence intervals. 
(A)

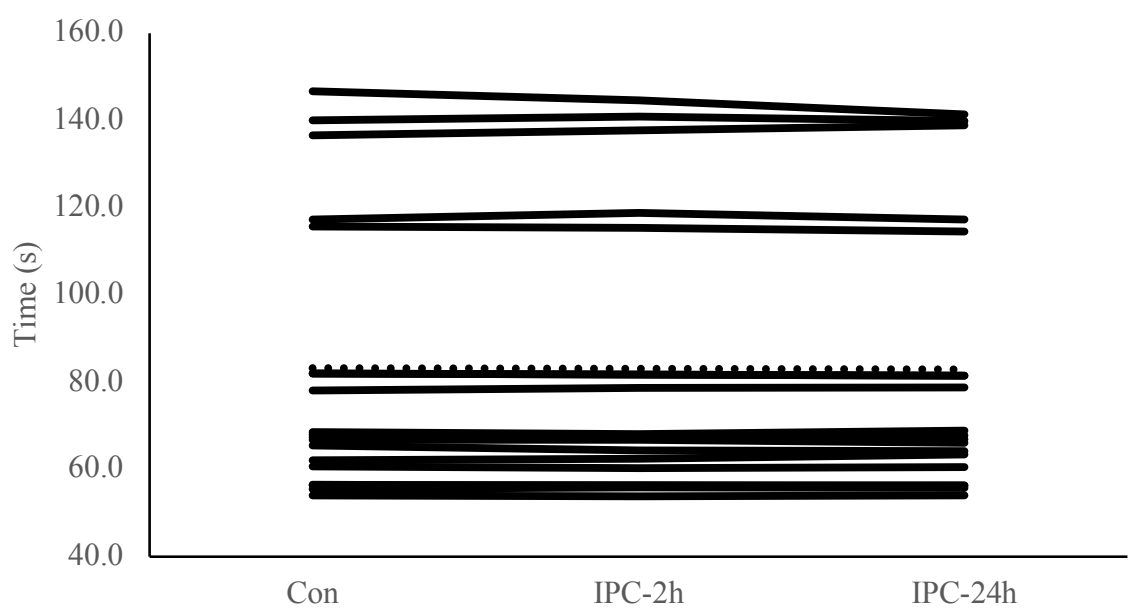

(B)

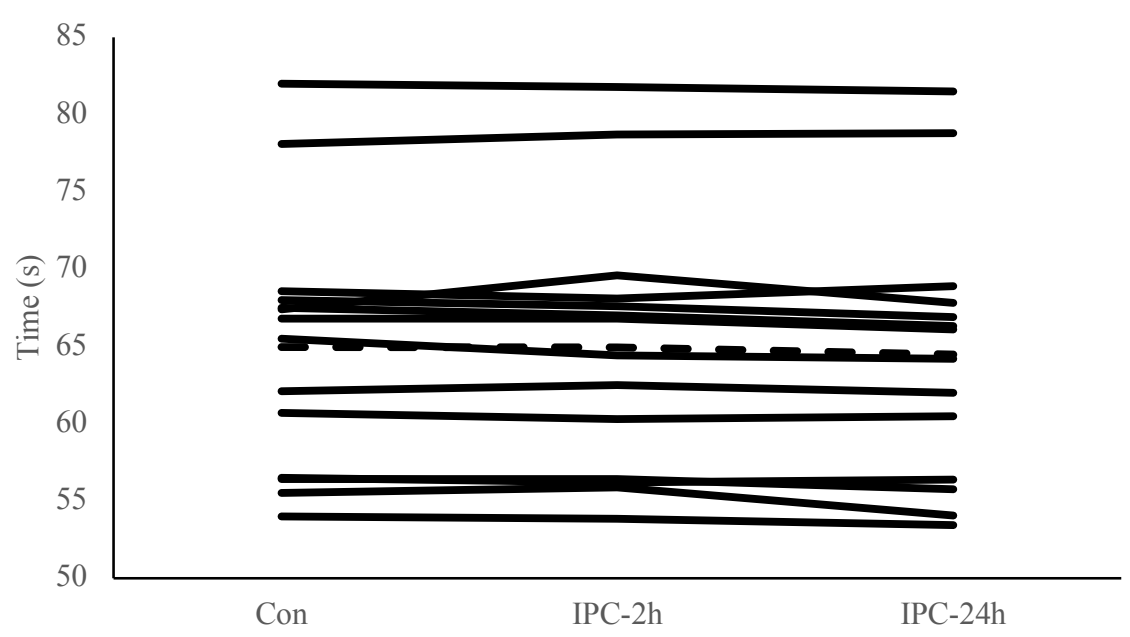

(C)

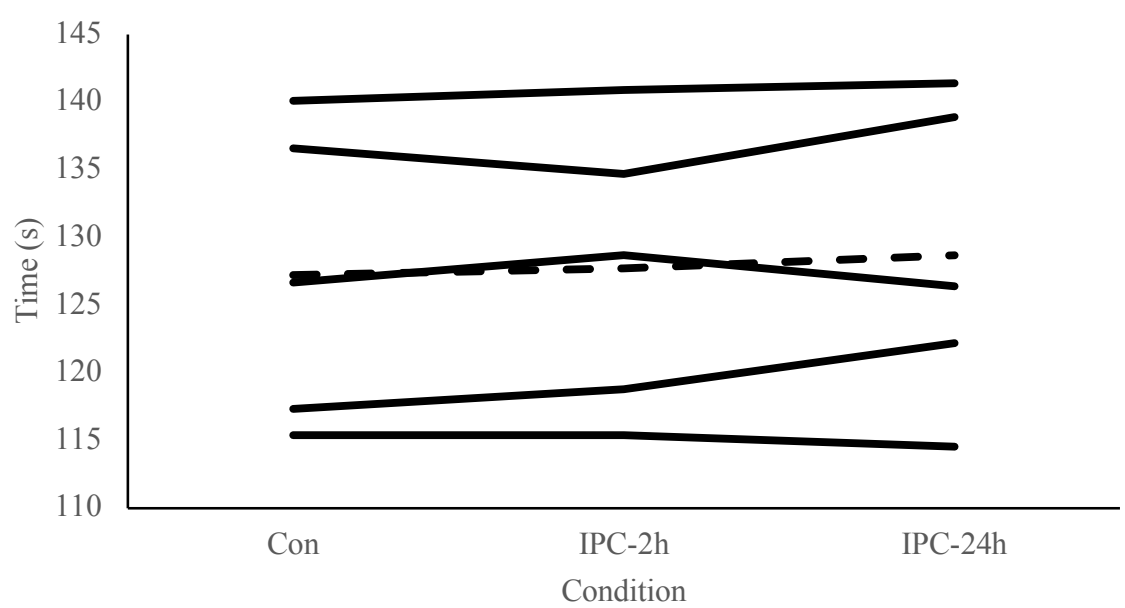

Figure 5.4: Individual responses of swimming time trial for the combined group (A), $100 \mathrm{~m}$ (B) and $200 \mathrm{~m}(\mathrm{C})$. IPC - ischemic preconditioning 
Table 5.3: Change in results between control and ischemic preconditioning applied $2 \mathrm{~h}$ prior to performance when compared to 0.3 of the coefficient of variation to determine smallest worthwhile change

\begin{tabular}{lll}
\hline 0.3 of CV $(\%)$ & Performance Con:IPC-2h $(\%)$ & SWC Y/N \\
\hline 0.00 & 0.00 & $\mathrm{~N}$ \\
0.13 & 0.59 & $\mathrm{Y}$ \\
0.14 & 0.66 & $\mathrm{Y}$ \\
0.36 & 1.68 & $\mathrm{Y}$ \\
0.16 & 0.73 & $\mathrm{Y}$ \\
0.17 & -0.81 & $\mathrm{~N}$ \\
0.12 & -0.57 & $\mathrm{Y}$ \\
0.11 & 0.53 & $\mathrm{Y}$ \\
0.05 & 0.24 & $\mathrm{~N}$ \\
0.16 & -0.77 & $\mathrm{Y}$ \\
0.29 & 8.18 & $\mathrm{Y}$ \\
0.16 & 0.74 & $\mathrm{~N}$ \\
0.00 & 0.00 & $\mathrm{~N}$ \\
0.06 & 0.00 & $\mathrm{~N}$ \\
0.14 & -0.68 & $\mathrm{~N}$ \\
0.27 & -1.26 & $\mathrm{Y}$ \\
0.06 & 0.30 & $\mathrm{~N}$ \\
0.14 & -0.64 & \\
CV - coefficient of variation; Con - control; IPC - ischemic preconditioning; SWC - smallest \\
worthwhile change; Y - yes; $\mathrm{N}-$ No
\end{tabular}


Table 5.4: Change in results between control and ischemic preconditioning applied $24 \mathrm{~h}$ prior to performance when compared to 0.3 of the coefficient of variation to determine smallest worthwhile change

\begin{tabular}{lll}
\hline 0.3 of CV $(\%)$ & Performance Con:IPC-24h $(\%)$ & SWC Y/N \\
\hline 0.22 & 1.05 & $\mathrm{Y}$ \\
0.35 & 1.62 & $\mathrm{Y}$ \\
0.07 & 0.33 & $\mathrm{Y}$ \\
0.43 & 1.98 & $\mathrm{Y}$ \\
0.09 & -0.44 & $\mathrm{~N}$ \\
0.35 & -1.68 & $\mathrm{~N}$ \\
0.03 & 0.14 & $\mathrm{Y}$ \\
0.04 & 0.18 & $\mathrm{Y}$ \\
0.13 & 0.61 & $\mathrm{Y}$ \\
0.19 & -0.9 & $\mathrm{~N}$ \\
0.78 & 3.61 & $\mathrm{Y}$ \\
0.09 & -0.44 & $\mathrm{~N}$ \\
0.04 & 0.18 & $\mathrm{Y}$ \\
0.21 & 0.99 & $\mathrm{Y}$ \\
0.10 & -0.47 & $\mathrm{~N}$ \\
0.01 & 0.03 & $\mathrm{Y}$ \\
0.02 & -0.11 & $\mathrm{~N}$ \\
0.45 & -2.16 & $\mathrm{~N}$ \\
\hline
\end{tabular}

$\mathrm{CV}$ - coefficient of variation; Con - control; IPC - ischemic preconditioning; SWC - smallest worthwhile change; $\mathrm{Y}$ - yes; $\mathrm{N}$ - No 


\subsection{DISCUSSION}

In this study, limb IPC did not improve 100 or $200 \mathrm{~m}$ swimming performance in National and international-level swimmers when applied $2 \mathrm{~h}$ or $24 \mathrm{~h}$ prior to performance assessment. These findings, particularly for IPC-2h, oppose previous research that found limb IPC applied acutely improved subsequent swimming performance (Jean-St-Michel et al. 2011; Lisbôa et al. 2017; Marocolo et al. 2015). Consistent with previous research (Seeger et al. 2017), no change in swimming performance was identified when limb IPC was applied $24 \mathrm{~h}$ before the TT when reporting combined distance results. Likewise, no differences were identified in physiological markers following IPC-2h or IPC-24h. Therefore, combined results of limb IPC, applied $2 \mathrm{~h}$ or 24 h, did not improve swimming performance or physiological markers.

For short duration events (i.e. 10-90 s), a recent meta-analysis showed that a longer duration between limb IPC and exercise resulted in a higher effect size; suggesting that limb IPC may be dependent on the timing of the preconditioning strategy relative to the start of subsequent performance (Salvador et al. 2016). Previous research in swimming has implemented limb IPC between $10 \mathrm{~min}$ and $8 \mathrm{~h}$ (Jean-St-Michel et al. 2011; Lisbôa et al. 2017; Marocolo et al. 2015) before performance assessment and found beneficial effects; findings which contradict those reported here when limb IPC was applied $2 \mathrm{~h}$ before exercise and furthermore the negative effect that was observed in the $200 \mathrm{~m}$ following both conditions compared to a control. However, it should be considered only 5 participants completed $200 \mathrm{~m}$, one of which decreased performance by $4 \%$ negatively skewing results. This large variation in performance is unusual for elite athletes, supported by research completed by Pyne et al. (2004) in which variation in performance is reported to be $0.8 \%$ between competitions. Therefore, additional unknown variables may be influencing this individual performance. Within the current results variation in individual performance was observed as displayed in Figure 5.4; the individual performance differences displayed 9 participants had a smallest worthwhile change in performance following the use of IPC-2h (Table 5.3), with variation in change required ranging from $0.00-0.29 \%$. Whereas 11 participants showed a smallest important change after completion of IPC-24h (Table 5.4) with a required change ranging from $0.01-0.45 \%$ when results were combined as a group. The individual variation in performance, although speculative may be consequence of different training backgrounds in which some athletes training has focussed more aerobically or anaerobically affecting the impact of limb IPC or potential impact of the differences in stroke technique, however no patterns have been identified within the current results providing support of this.

Alternatively, several methodological differences between the present study and previous literature may explain this lack of agreement in findings. Specifically, there is little consensus regarding 
optimal cuff pressures used in limb IPC as different pressures have been reported (i.e., 200-230 $\mathrm{mmHg}$ or $15-50>\mathrm{SBP}$ ) which are universally applied across all individuals within previous studies. This is an important consideration as the percentage of blood flow restriction may affect the success of limb IPC as a pre-competition strategy (Hargens et al. 1987). A standardised cuff pressure may not cause the same percentage of blood flow restriction in every individual, which was highlighted as a limitation when completing a review of the literature and was not practical to apply into elite sport. In some instances cuff pressure has been individualised according to systolic blood pressure as previous reports have recommended pressures to be higher than blood pressure (Horiuchi 2017; Pope et al. 2013), however the volume and type of tissue surrounding the blood vessels may influence the pressure exerted on the vasculature (Loenneke et al. 2012). Recent research by Loenneke et al. (2015) recommended the use of individualised cuff pressure calculated from thigh girth and resting blood pressure, which was adopted in the current study. However, blood flow restriction was not confirmed using a Doppler (e.g. Laurentino et al. 2008), due to practicality, which offers a limitation to the current study as limb IPC was individualised from estimated calculations rather than according to a measured pressure. This may be an attributing factor to individual variation identified in performance as some participants may have experienced more or less blood flow restriction, consequently affecting mechanisms hypothesized to be beneficial to swimming performance. Determination of the most suitable protocol for cuff pressure needs to be established, investigating comparisons between standardised cuff pressures, the use of thigh girth and blood pressure to calculate individual pressures and Doppler assessment for required percentage of blood flow restriction are required. The results of these three methods to determine cuff pressure need to be identified and the resultant effect on performance tested to establish recommendations for practical use.

To explain the current results, another methodological difference should be considered regarding the location of the cuff, with application previously reported on the lower or upper body. The present study applied occlusion cuffs to the thighs which contrasts previous research in swimming whereby cuffs were applied to the upper body (Jean-St-Michel et al. 2011; Lisbôa et al. 2017; Marocolo et al. 2015). Although limited research still exists on the working mechanism of limb IPC and athletic performance, it has been suggested that limb IPC induces a systemic change in blood flow through a change in sympathetic activity; some studies suggest a humoral hypothesis postulating that an ischemic event leads to the production of substances that could reach another tissue (Marocolo et al. 2016). It has been hypothesized that limb IPC may have potential to improve exercise performance via both local effects (i.e. to the limb) and remote effects (via cardiovascular or nervous system). Due to the nature of swimming and controlled breathing, which can result in exercise induced arterial hypoxemia, decreased pH (Craig 1986; Sharp et al. 1991) and consequently a significant contributor of fatigue (Noakes 2000), a systemic increase in blood 
flow and $\mathrm{O}_{2}$ delivery could be speculated to improve performance, reducing hypoxemia and metabolic acidosis. However, in the current study no differences were identified between conditions in the physiological measures. Alternative research has suggested that limb IPC may also cause local changes in the muscle at the site of the cuff (e.g. increase $\mathrm{O}_{2}$ uptake or change in mitochondrial activity) which may contribute to an increase muscle oxygenation (Horiuchi et al. 2015; Kjeld et al. 2014; Saito et al. 2004). In swimming, the contribution of propulsive force is approximately 90\% for the upper extremities (Deschodt et al. 1999; Morouço et al. 2011), therefore, the local changes achieved by application of the cuffs to the upper limbs, may increase effectiveness of limb IPC to improve swimming performance. In comparison to previous results, applying cuffs to the thighs to induce a systemic response, this may help to explain the inconsistency in the current results. Consistent with the current results, Lalonde \& Curnier (2014) reported that remote IPC on the arm did not improve exercise performance during a Wingate test. Whereas remote IPC on the leg has previously improved time to failure during rhythmic handgrip exercise (Barbosa et al. 2015). Interestingly, despite the significant benefits in exercise tolerance, no differences in physiological variables, such as blood flow, vascular conductance, and muscle deoxygenation profiles, were observed between IPC and CON in the same study. However, no other studies have directly compared the effects of different locations with limb IPC on exercise performance; thereby, it is difficult to derive a conclusion. Consequently, this is an area that warrants further investigation to determine the impact of systemic versus local blood flow restriction on athletic performance.

To date, one study has examined the use of limb IPC applied $24 \mathrm{~h}$ prior to performance to determine if the late phase of limb IPC, originally used in a clinical setting, may also improve athletic performance. The current study replicated research complete by Seeger et al. (2017) but within swimming, with the only other methodological difference being individualising cuff pressures. Similarly, no difference in performance time between conditions was identified. However, results from the current study were not consistent with previous research investigating limb IPC in swimming, previously a benefit has been identified in the early phase (10 min $-2 \mathrm{~h}$ ) within the literature which was not consistent in our study. Additional physiological measures may have delineated the results to determine if limb IPC affected endothelial dysfunction (through measurement of FMD), as one of the predominant mechanisms influencing performance. Limb IPC has shown to prevent endothelium dysfunction (Dawson et al. 2013; Bailey et al. 2012), increasing aerobic contribution to performance. Considering reported aerobic contribution in swimming this mechanism was deemed important. Loukogeorgakis et al. (2005) reported RIPC applied immediately before induced ischemia, prevented endothelial dysfunction (FMD before 9.4\% and after $8 \%$ ) and also applied $24 \mathrm{~h}$ prior, FMD was preserved and prevented dysfunction ( $8.7 \%$ before and $8.4 \%$ after). Due to practicality, FMD was unable to be measured and therefore 
only speculative as to whether this mechanism was not influenced by the limb IPC protocol. As suggested methodological differences could have influenced these findings as stated above regarding cuff pressure and location, consequently limb IPC applied $24 \mathrm{~h}$ prior to performance should be further investigated in swimming while ensuring that cuffs are applied to the upper body and where practically possible complete additional physiological measures to delineate why large individual differences occur.

Limitations of the research should be acknowledged; the placebo effect is not accounted for, which may be an important factor as previous research has highlighted the ability of the placebo effect to improve performance (Cook \& Beaven 2013). Within Con, cuffs were still applied which was still novel in comparison to lying and resting with the absence of cuffs and it is difficult to blind participants from the conditions due to the obvious difference in cuff pressure. Therefore, a further condition would be optimal to identify the impact of vascular occlusion, determining whether there is a placebo or physiological effect on performance when compared against a control in which cuffs are absent.

\subsection{PRACTICAL APPLICATIONS}

Despite this study concluding swimming performance was not improve following limb IPC applied at $2 \mathrm{~h}$ or $24 \mathrm{~h}$, there are several practical points of relevance for application in sport. These results provide baseline data for the use of limb IPC in swimming when cuffs are applied to the thighs, identifying that this strategy had no detrimental effect on physiological responses. Most prominently, the combination of previous research and the current study suggest recommendations for application of the cuffs to the upper body to improve swimming performance.

\subsection{CONCLUSION}

In conclusion, the current study demonstrated swimming TT performance of 100 or $200 \mathrm{~m}$ was not improved when it was preceded $2 \mathrm{~h}$ or $24 \mathrm{~h}$ by four cycles of limb IPC, at an individualized cuff pressure. Speculatively, this may have been due to the difference in cuff placement on the lower limbs as opposed to upper limbs as in previous IPC and swimming research. Therefore, the use of limb IPC $24 \mathrm{~h}$ prior to swimming TT performance should be investigated with cuffs applied to the upper limbs to identify if the late phase of limb IPC can also improve performance, as this would have greater practical application completing the limb IPC protocol $24 \mathrm{~h}$ before competition rather than in close proximity to the start of an athletic event. 


\section{Chapter 6 - The effect of lower limb occlusion on recovery following sprint exercise in academy rugby union players}

\subsection{STUDY INFORMATION}

In contrast to the previous experimental chapters, the following study focuses on the use of vascular occlusion as a recovery strategy. Short term fatigue resulting from athletic performance has been negatively linked to subsequent performance, therefore recovery is an integral part of training and competition. Consequently, recovery strategies are frequently applied to increase the rate of recovery to optimise performance. Many researched strategies are limited in applied practice due to cost, equipment and time, as a result alternative options are pursued by performance scientists to implement in elite sport. As highlighted in the introduction and review of literature, vascular occlusion offers an alternative recovery modality, but requires further investigation prior to realisation in sport.

Aim: Investigate the effects of vascular occlusion on recovery rate $(2 \mathrm{~h}$ and $24 \mathrm{~h})$ using individualised cuff pressure, on physiological and neuromuscular assessment following maximal sprint exercise, whilst also considering hormonal reactivity to a subsequent exercise challenge performed at $24 \mathrm{~h}$.

Objectives:

- To determine the effect of vascular occlusion, as a recovery strategy, on physiological markers previously used within the literature $(\mathrm{CK}, \mathrm{T}, \mathrm{C}, \mathrm{NMF}$, perception muscle soreness)

- To identify if vascular occlusion improves recovery time of physiological measures after $2 \mathrm{~h}$ and $24 \mathrm{~h}$ to coincide with the timeline of second rounds of competition and/or return to training

- To identify if a physical stress test performed after $24 \mathrm{~h}$ provides further denote if players are adequately recovered to return to training/preparedness for next competition

Hypothesis: Application of vascular occlusion following repeated sprints to induce muscle damage (i.e. post exercise) would facilitate the recovery of biochemical, neuromuscular and hormonal markers measured after $24 \mathrm{~h}$

Outcomes measures: CK, T, C, PPO, perception muscle soreness and recovery 


\subsection{INTRODUCTION}

Physical and metabolic disturbances result from team sport match-play (Bangsbo et al. 2006). Accordingly, various measures are used in applied practice to indicate the presence of EIMD and the efficacy of recovery interventions (Ferguson et al. 2014). Elevated CK concentrations (Cunniffe et al. 2011; Takarada 2003; Russell et al. 2016), disruption in the $\mathrm{T}$ and $\mathrm{C}$ hormonal milieu (Shearer et al. 2015), and impairments in NMF occur post-match (West et al. 2014); with measurements showing perturbation for at least $48 \mathrm{~h}$ after (Russell et al. 2016; West et al. 2014). The use of hormonal responses as additional surrogate markers for readiness-to-train or competition-preparedness is an emerging concept in the recovery paradigm to help indicate levels of fatigue (Crewther et al. 2017). The reasoning for measuring hormonal response is associated with free $\mathrm{T} / \mathrm{C}$ ratio providing a diagnostic or preventative test to detect overtraining as it gives an indication of the anaerobic/catabolic balance in response to training and competition, which are known to affect the recovery state and duration of recovery after exercise (Fry et al. 1991).

As short-term fatigue after match-play has been negatively linked to subsequent performance, recovery strategies are an integral component in the weekly training regimen (Kellmann 2010) especially as multiple games are played in the week. The post-exercise recovery effects of cold water immersion, contrast water therapy, active recovery, neuromuscular electrical stimulation and compression garments have been investigated (for review see Nédélec et al. 2013). However, many of these strategies have limitations for application due to required equipment, space, time and cost, with literature also reporting contrasts in the effectiveness of each of these strategies. Ice baths for instance decrease muscle temperature which may be negative for multiple efforts or games performed in one day of competition. Also, athlete perception of recovery strategies is key, it is known that players' beliefs of the efficacy of an intervention can influence subsequent responses (Cook \& Beaven 2013) therefore knowledge of all available strategies and efficacy on recovery rate are important for performance scientists and achieving success of implementing recovery strategies to improve performance.

More recently, vascular occlusion (the use of blood pressure cuffs applied on specific limbs to restrict blood flow) has been suggested as a recovery strategy (Beaven et al. 2012; Northey et al. 2016; Garcia et al. 2017; Page et al. 2017; Borne et al. 2016) (section 2.6). While the exact mechanisms are unclear, vascular occlusion is purported to elevate adenosine concentrations and activation of $\mathrm{K}_{\mathrm{ATP}}$, increasing blood flow (Goto et al. 1995) and benefiting $\mathrm{O}_{2}$ and nutrient delivery via vasculature dilation; a response likely exaggerated during reperfusion, possibly improving substrate re-synthesis (Byrne et al. 2004). Alternatively, Konstantinov et al. (2004) showed diminished expression of inflammatory genes in neutrophils following limb IPC. Since 
neutrophils are one of the key mediators of IRI responsible for oxidative stress and injury to organs, modulation of neutrophil activation is important to reduce IRI. Furthermore, attenuated inflammatory responses and reductions of muscle oedema and intramuscular pressure decrease nocioreceptor stimulation, potentially reducing muscle soreness (Howatson \& van Someren 2008).

Unfortunately, the evidence for vascular occlusion as an effective post-exercise recovery modality is currently inconsistent. Two investigations have identified improvements in recovery (Beaven et al. 2012; Page et al. 2017) whereas others disagree (Garcia et al. 2017; Northey et al. 2016; Borne et al. 2016) (refer to section 2.6.1). This disparity may be explained by methodological differences in the implementation of vascular occlusion, variance in participant activity levels and variability in the stimulus for EIMD. Borne et al. (2016) applied 24 min of continual pressure using elastic wraps versus the intermittent application of pressure for $12 \mathrm{~min}$ in other studies showing accelerated recovery (Beaven et al. 2012). Moreover, standardised cuff pressures have been used irrespective of participant characteristics despite recommendations regarding individualisation of cuff pressures relative to thigh girth and resting blood measurements (Loenneke et al. 2015). Similarly, inconsistencies in the timing of recovery markers (1-72 $\mathrm{h}$ post occlusion) have occurred between studies. Previous research has also required players to remain rested for the duration of post exercise recovery; this has limited application to applied practice where football or rugby players are frequently required to play multiple games within a week (i.e $<72 \mathrm{~h}$ separating games) and train when complete physical recovery may not be achieved (Nédélec et al. 2012). Accordingly, identification of the physiological response to a subsequent physical stressor may denote if players are adequately recovered to return to training. Notably, stress induced hormone reactivity can provide an indication of the anabolic/catabolic balance in response to training and competition (Adlercreutz et al. 1986). Specifically, $\mathrm{T}$ is a stress biomarker; consequently, the monitoring of $\mathrm{T}$ in response to a physical stress test could provide information on readiness to train/compete (Crewther \& Cook 2012). Rapid bioavailability of $\mathrm{T}$ and effects on CNS, such that human behaviour towards physical performance may be modified through acute perturbations (Crewther et al. 2011) has been related to increased winning (vs losing); Crewther et al. (2017) identified a larger $\mathrm{T}$ response to a mid-week stress test before winning. Thus, the primary aim of the study was to investigate the effects of vascular occlusion on recovery rate $(2 \mathrm{~h}$ and $24 \mathrm{~h}$ ), using individualised cuff pressure, on physiological and performance assessment following maximal sprint exercise, whilst also considering hormonal reactivity to a subsequent exercise challenge performed at $24 \mathrm{~h}$. 


\subsection{METHOD}

\subsubsection{Experimental overview}

Within rugby union, a crossover, randomised control trial allowed for examination of vascular occlusion applied as a recovery strategy, after completion of repeated sprints. The study consisted of two interventions, separated by seven days; Control (Con) and vascular occlusion (Recovery). In both conditions, a blood and saliva sample were completed along with $\mathrm{CMJ}$ and subjective ratings of recovery and muscle soreness prior to completing six $50 \mathrm{~m}$ sprints. Measures were repeated prior to application of cuffs on the most proximal point of the thighs, and inflated to either $15 \mathrm{mmHg}$ (Con) or an individualised cuff pressure (Recovery) for a total of $12 \mathrm{~min}$. At $2 \mathrm{~h}$ and 24 $\mathrm{h}$ measures were repeated with the addition of a physical stress test performed at $24 \mathrm{~h}$, after which an additional saliva sample was provided to assess hormone reactivity (Figure 6.1).

\subsubsection{Participants}

Following ethical approval and informed written consent being obtained, twenty-four male professional rugby union academy players (age: $21.8 \pm 3.0 \mathrm{y}$, mass: $96.9 \pm 10.1 \mathrm{~kg}$, stature: $1.85 \pm$ $0.09 \mathrm{~m}$ ) participated in the study during pre-season (1-2 sessions per day, 4-5 days a week) including strength, power and speed training sessions which were stable and consistent the week prior to and during the two weeks when trials were performed. All participants were informed of the experimental procedures and the purpose of the study, along with possible risks.

Participants attended the testing venue on four occasions. Main trials were performed inside on a $3 \mathrm{G}$ surface to minimise environmental influences (temperature $20^{\circ} \mathrm{C}$; humidity $41 \%$ ). To limit circadian variation, measurement timings were consistent between trials and participants refrained from alcohol and intense physical exercise in the $24 \mathrm{~h}$ preceding trials. No exercise was permitted between days one and two of testing.

\subsubsection{Main trial procedures}

On arrival for main trials, participants rested for $10 \mathrm{~min}$ before blood pressure was recorded (Omron Healthcare, Europe; systolic $>140 \mathrm{mmHg}$ and/or diastolic $>90 \mathrm{mmHg}$ precluded further study involvement). Thigh girth was measured for determination of cuff pressure and physiological (capillary blood and saliva) and perceptual (soreness and recovery) assessments followed. After a 10-min standardised warm-up, participants performed two maximal effort CMJ separated by $90 \mathrm{~s}$ (portable force platform: Type 92866AA, Kistler, Germany) to assess NMF. A further $10 \mathrm{~min}$ warm-up followed (20 m dynamic exercises and acceleration sprints, two $50 \mathrm{~m}$ sprints at $80 \%$ and $100 \%$ effort) with five min of enforced rest before six $50 \mathrm{~m}$ (each separated by 
five min rest) timed sprints (Brower Timing System, Salt Lake City, Utah, USA) were performed to induce muscle damage (Taylor et al. 2014; Johnston et al.2015). This was performed as a group; however, participants ran separately to prevent competition influencing competition time. Instruction prior to both sessions explained maximal effort was required across all six sprints, no encouragement was provided during the sprints with coaches and participants blinded from time of completion to all sprints until both sessions were completed. Average time and cumulative sprint times for the six sprints were recorded.

Immediately post-exercise, all baseline measures were repeated (within 5 min of sprints finishing) before occlusion cuffs were applied to the most proximal point of the thighs while participants lay supine. The cuff was manually inflated to $15 \mathrm{mmHg}$ (Con), this reflects previous research (Beaven et al. 2012), or to $60 \%$ of individually calculated pressures (171 to $266 \mathrm{mmHg}$; Recovery), determined from thigh girth and blood pressure measurements (Loenneke et al. 2015) (see section 3.3-3.5). Cuffs were applied for a total of $12 \mathrm{~min}$ (two cycles of three min occlusion, three min reperfusion). The rationale behind this protocol, as reported by (Beaven et al. 2012), identified three min cycles of occlusion and reperfusion fulfil the duration threshold criterion that has been reported previously (for further information see section 2.6). After $2 \mathrm{~h}$ and $24 \mathrm{~h}$ baseline measures were repeated, consistent with previous research (Taylor et al. 2015; Johnston et al. 2015) and are representative of the time between competition and return to training.

To assess hormonal reactivity to a subsequent exercise stimulus performed after $24 \mathrm{~h}$, saliva was collected five min before and immediately after the completion of a 30-min physical stress test (three sets of power cleans and back squats, and four sets of bench press and bench pull at relative loads of 60 to $85 \% 1 \mathrm{RM}$; one and three min rest between sets and exercises, respectively). Participants were accustomed to resistance exercise from their normal training and testing procedures and these protocols were deemed sufficient to elicit a stress response (Crewther \& Cook 2012). Coaches supervising the sessions were not aware of the conditions participants had completed $24 \mathrm{~h}$ prior and provided technical support only. Feedback was not provided regarding within-session performance.

Saliva collection was completed as detailed in Chapter 3, section 3.7. Participants provided a 20 $\mu \mathrm{L}$ fingertip capillary blood sample for blood lactate analysis and a $120 \mu \mathrm{L}$ sample was collected for analysis of CK. Refer to Chapter 3, section 3.6.3 for details on timings, sample preparation and analysis. 


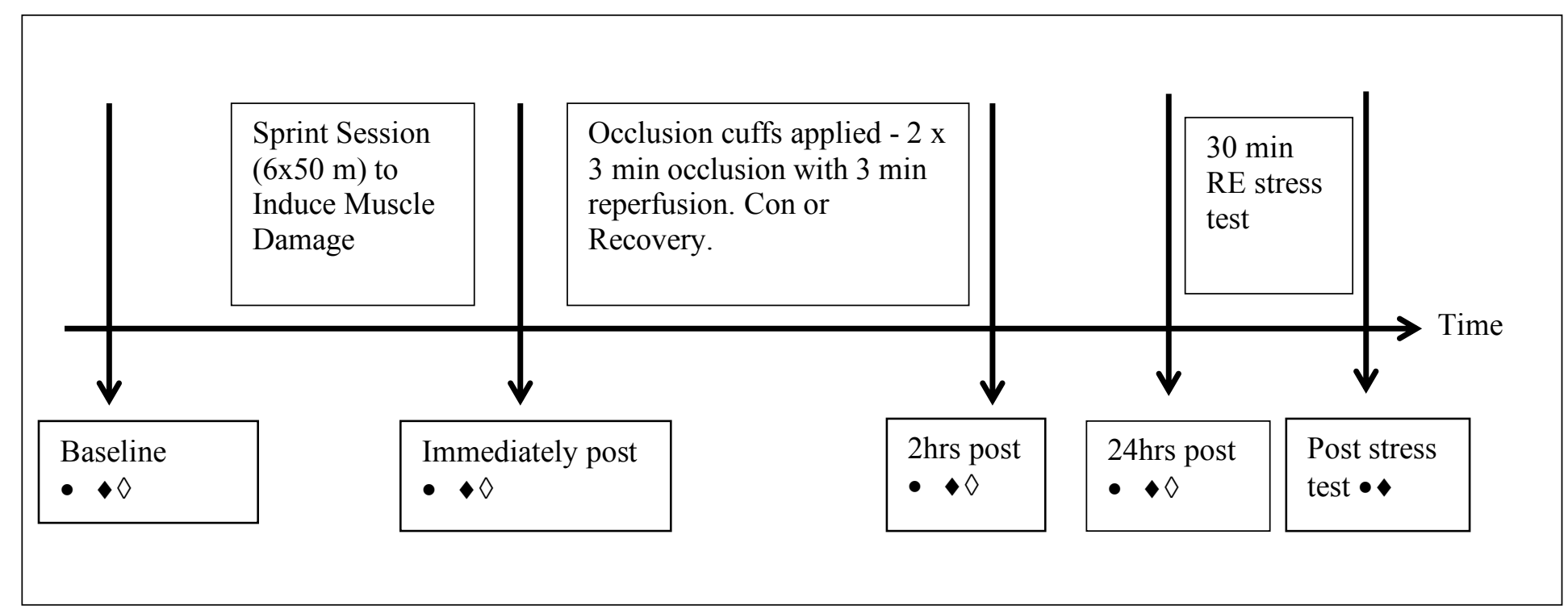

Figure 6.1: Timeline of data collection. Con; control trial, Recovery; occlusion trial, RE; resistance exercise. Measurements: salivary testosterone, salivary cortisol $(\bullet)$, blood sampling for blood lactate and Creatine Kinase ( ), perception muscle soreness questionnaires $(\bullet)$, countermovement jump $(\diamond)$ 
Perceived lower body muscle soreness was assessed using a 7-point Likert scale (Appendix G) ranging from 0 (complete absence of soreness) to 6 (severe pain that limits movement) which is reliable and valid (Impellizzeri \& Maffiuletti 2007). Perception of recovery status was assessed using a 11-point likert scale (Appendix H) (Laurent et al. 2011) organised from 0 (very poorly recovered/extremely tired) to 10 (very well recovered/highly energetic) which reflects changes in total sprint time relative to prior exercise (Laurent et al. 2011). Participants were familiar with the scales and were asked to base scores on perceived soreness during normal movement and were alone when recording scores to reduce the influence of peers. Assessment of CMJ performance was completed on a portable force platform (Kistler instrument Ltd., Farnborough, UK), analysis was completed as described in section 3.8 to calculate PPO and $\mathrm{JH}$.

\subsubsection{Statistical analysis}

All data is presented as mean $\pm \mathrm{SD}$. Following confirmation of parametric assumptions, repeated measures MANOVA with Bonferroni adjustment assessed between-trial differences for variables with multiple time points per trial (i.e. T, C, T:C ratio, perception muscle soreness and recovery, $\mathrm{CK}$, blood lactate, PPO and $\mathrm{JH}$ ). A one-way ANOVA was performed to assess between-trial differences in response to the physical stress test (T, C, T:C ratio and perception muscle soreness). Paired samples t-tests were performed for between-trial comparisons of data expressed over a single time point within a trial (i.e. mean and total sprint times, $\mathrm{T}$ and $\mathrm{C}$ pre-and post-stress test). For further detail see Chapter 3, section 3.9.

\subsection{RESULTS}

There were no significant differences between conditions for total (Recovery: $39.86 \pm 2.87 \mathrm{~s}$; Con: $40.26 \pm 2.77 \mathrm{~s}, \mathrm{p}=0.238$ ) or average (Recovery: $6.69 \pm 0.47 \mathrm{~s}$; Con: $6.71 \pm 0.46 \mathrm{~s}, \mathrm{p}=0.674$ ) sprint times. The $\Delta$ lactate concentrations from pre-to-post sprinting also showed no significant difference between conditions (Recovery: $6.88 \pm 2.53 \mathrm{mmol} \cdot \mathrm{L}^{-1}, 85 \pm 6 \%$; Con: $6.76 \pm 2.04$ $\mathrm{mmol} \cdot \mathrm{L}^{-1}, 86 \pm 5 \%, \mathrm{p}=0.807$, Figure 6.2$)$.

There was a significant time effect for $\mathrm{CK}\left(\mathrm{F}_{(1,48)}=72.928, \mathrm{p}<0.001\right.$, Figure 6.2) with significant increases at $24 \mathrm{~h}$ compared to pre-sprints in both Recovery $\left(408.19 \pm 291.45 \mu \cdot \mathrm{L}^{-1}, 55 \pm 33 \%\right.$, $\mathrm{p}<0.001)$ and $\operatorname{Con}\left(506.02 \pm 359.14 \mu \cdot \mathrm{L}^{-1}, 56 \pm 34 \% \mathrm{p}<0.001\right)$. However, there was no significant interaction effects between condition and time $\left(\mathrm{F}_{(1,48)}=1.157, \mathrm{p}=0.293\right)$.

Muscle soreness $\left(F_{(2,95)}=7.714, p<0.001\right)$ and perception of recovery $\left(F_{(2,88)}=70.931, p<0.001\right)$ were affected by sprinting, with the greatest change in muscle soreness occurring $24 \mathrm{~h}$ post exercise (Recovery: $1.5 \pm 1.0$; Con: $1.6 \pm 1.1$ ). There was no significant interaction effects between time 
and condition for muscle soreness $\left(\mathrm{F}_{(2,95)}=0.009, \mathrm{p}=0.993\right)$ or perception of recovery $\left(\mathrm{F}_{(2,88)}=0.158\right.$, $\mathrm{p}=0.924)$.

Sprint exercise affected PPO $\left(\mathrm{F}_{(2,96)}=42.141, \mathrm{p}<0.001\right)$ and $\mathrm{JH}\left(\mathrm{F}_{(2,82)}=58.353, \mathrm{p}<0.001\right)$ with PPO (Recovery: $417.74 \pm 293.09 \mathrm{~W}, 8 \pm 4 \%, \mathrm{p}<0.001$; Con: $401.46 \pm 332.69 \mathrm{~W}, 7 \pm 5 \%, \mathrm{p}<0.001$ ) and JH (Recovery: $5.49 \pm 3.24 \mathrm{~cm}, 13 \pm 7 \%$, p $<0.001$; Con: $5.38 \pm 3.66 \mathrm{~cm}, 13 \pm 8 \%, \mathrm{p}<0.001$ ) decreasing immediately post-sprints. No further timing effects and no effect of Recovery on PPO $\left(\mathrm{F}_{(2,96)}=0.304, \mathrm{p}=0.757\right)$ or $\mathrm{JH}\left(\mathrm{F}_{(2,82)}=0.304, \mathrm{p}=0.436\right)$ occurred.

Sprinting increased $\mathrm{T}\left(\mathrm{F}_{(2,100)}=20.127, \mathrm{p}<0.001\right.$, Figure 6.3), whilst a decrease in $\mathrm{T}, \mathrm{C}\left(\mathrm{F}_{(2}\right.$, $\left.{ }_{89)}=32.651, \mathrm{p}<0.001\right)$ and increase in $\mathrm{T}: \mathrm{C}$ ratio $\left(\mathrm{F}_{(2,95)}=19.200, \mathrm{p}<0.001\right.$; Figure 6.3) occurred $2 \mathrm{~h}$ post sprinting. However, condition did not affect the recovery of $\mathrm{T}\left(\mathrm{F}_{(2,100)}=2.159, \mathrm{p}=0.114\right), \mathrm{C}$ $\left(\mathrm{F}_{(2,89)}=0.640, \mathrm{p}=0.531\right)$ or $\mathrm{T}: \mathrm{C}$ ratio $\left(\mathrm{F}_{(2,95)}=0.299, \mathrm{p}=0.759\right)$.

Testosterone significantly changed pre-to post, in response to the physical stress test in the Recovery $\left(+21.58 \pm 44.90 \mathrm{pg} \cdot \mathrm{ml}^{-1}, 7 \pm 17 \%, \mathrm{p}=0.031\right)$ but not the Con $\left(+10.62 \pm 36.57 \mathrm{pg} \cdot \mathrm{ml}^{-1}, 4\right.$ $\pm 13 \%, \mathrm{p}=0.178)$ with no differences in baseline values between conditions $(\mathrm{p}=0.232)$; however, $\Delta \mathrm{T}$ was not significantly different between conditions $(\mathrm{p}=0.109)$. Cortisol declined over time $\left(F_{(1,46)}=7.806, p<0.001\right)$, pre-to post physical stress test (Recovery: $-0.14 \pm 0.23 \mu \mathrm{g} \cdot \mathrm{dl}^{-1}, 38 \pm 72 \%$, $\mathrm{p}=0.007$; Con: $\left.-0.17 \pm 0.27 \mu \mathrm{g} \cdot \mathrm{dl}^{-1}, 50 \pm 94 \%, \mathrm{p}=0.006\right)$, with similar results for $\mathrm{T}: \mathrm{C}$ ratio $\left(\mathrm{F}_{(1}\right.$, $\left.{ }_{46)}=29.836, \mathrm{p}<0.001\right)$. Recovery had no impact on hormonal response as no differences were observed between conditions $(T ; p=0.226, C ; p=0.679, T: C ; p=0.421)$. 
(A)

(B)
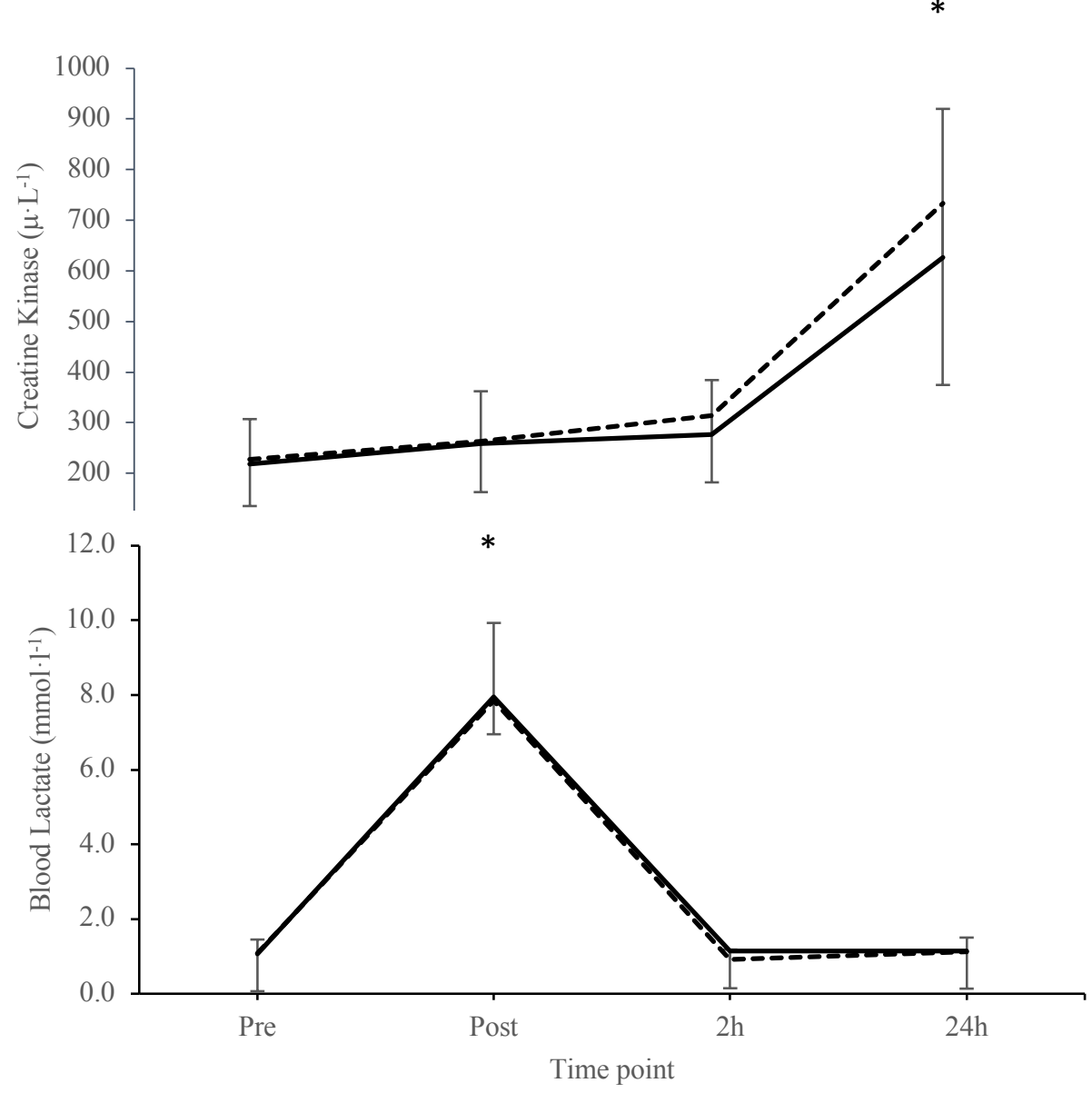

- Recovery ----.-Control

Figure 6.2: Blood markers (A) Creatine Kinase (B) Blood lactate collected pre, post, $2 \mathrm{~h}$ and 24 h post sprint protocol $*(\mathrm{p}<0.001)$ 
(A)

(B)

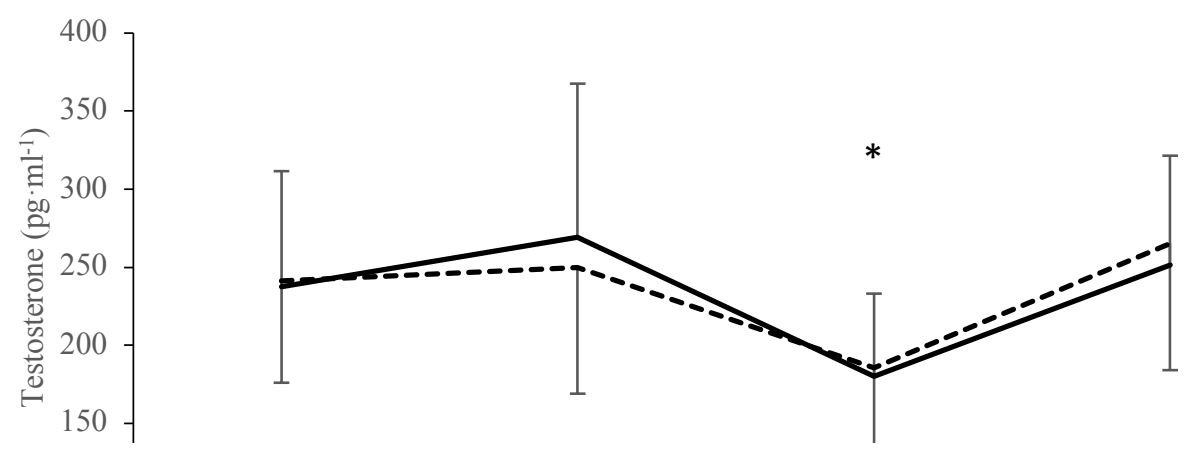

(C)
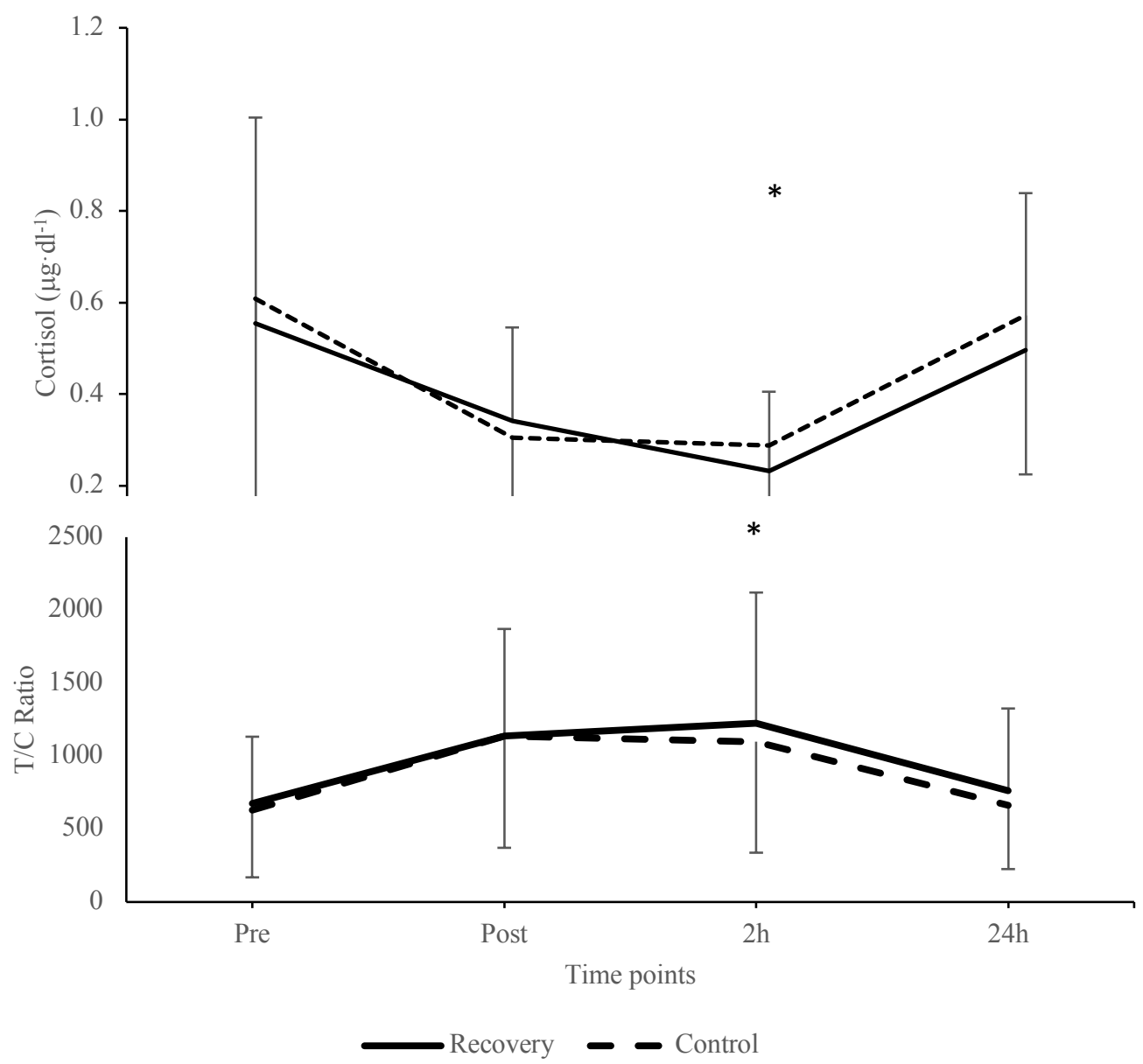

Figure 6.3: Saliva markers (A) Testosterone, (B) Cortisol (C) T/C ratio, collected pre, post, $2 \mathrm{~h}$ and 24 h post sprint $*(\mathrm{p}<0.001)$ 
(A)

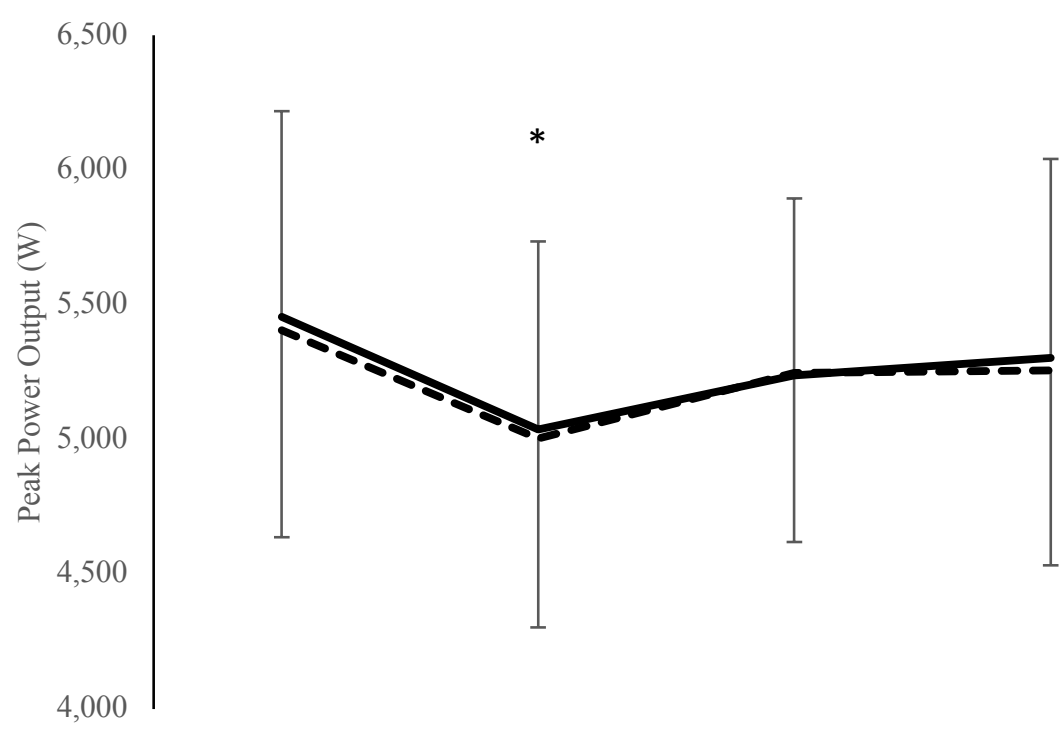

(B)

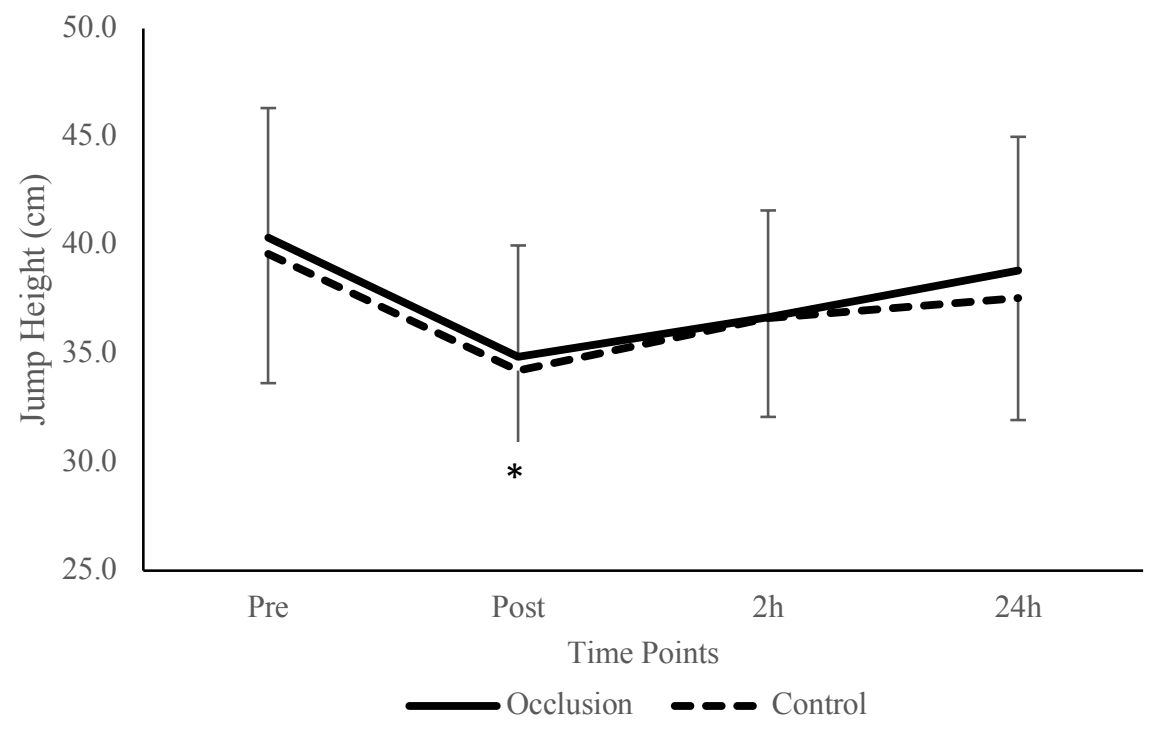

Figure 6.4: Neuromuscular function variables determined from countermovement jump (A) peak power output, (B) jump height, collected pre, post, $2 \mathrm{~h}$ and $24 \mathrm{~h}$ post sprint $*(\mathrm{p}<0.001)$ 


\subsection{DISCUSSION}

The primary aim of this study was to investigate the effects of vascular occlusion on recovery rate ( $2 \mathrm{~h}$ and $24 \mathrm{~h}$ ), using individualised cuff pressures, on physiological and neuromuscular indices following sprint exercise, while also considering hormonal reactivity to subsequent exercise. Vascular occlusion did not influence the physiological or neuromuscular markers measured in the $2 \mathrm{~h}$ or $24 \mathrm{~h}$ after sprint exercise in rugby union players. Repeated sprints were consistent across both trials (Recovery: $39.84 \pm 2.87$; Con: $40.26 \pm 2.77 \mathrm{~s}$ ) and induced EIMD in both conditions reflected by the perturbations in physiological (e.g. CK, Recovery: $55 \pm 33 \%$; Con: $56 \pm 34 \%$ ) and neuromuscular (e.g. PPO, Recovery: $8 \pm 4 \%$; Con: $7 \pm 5 \%$ ) responses, consistent with previous research (refer to section 2.2.4). Perception of muscle soreness was not different between conditions, sprinting increased muscle soreness $24 \mathrm{~h}$ post exercise. Moreover, no significant differences existed in response to a physical stress test at $24 \mathrm{~h}$ between conditions. Therefore, within the current study, vascular occlusion did not facilitate recovery following $2 \mathrm{~h}$ and $24 \mathrm{~h}$ of rest, nor change the hormonal response to a subsequent physical stress test. Likewise, it is notable that vascular occlusion did not have a detrimental effect on any physiological or neuromuscular markers assessed, recovery rate was not negatively influenced in comparison to Con, thus alleviating any concerns of detriment using this ergogenic strategy, acutely, within season.

Total $(\mathrm{p}=0.238)$ and average $(\mathrm{p}=0.674)$ sprint times were consistent between conditions with similar physiological responses being observed. Johnston et al. (2015) highlighted CK values increased by $570 \mu \cdot \mathrm{L}^{-1}$ (current results $+506.02 \mu \cdot \mathrm{L}^{-1}$ ) compared to match responses, increases of $586.6 \mu \cdot \mathrm{L}^{-1}\left(24 \mathrm{~h}\right.$ post soccer match) and $431 \mu \cdot \mathrm{L}^{-1}(16 \mathrm{~h}$ post rugby match) have been reported. However, time and distance of maximal sprint completed in a match cannot be controlled and varies depending on position, therefore comparison against match outcome is difficult as many factors may influence performance on match day. Nevertheless, previous reports suggest that six $50 \mathrm{~m}$ sprints reflect normal training sessions (Francis, 2008).

The current study individualised cuff pressure $(171-226 \mathrm{mmHg})$ as a standard pressure applied to different individuals may non-uniformly influence the pressure exerted on the vasculature and thus impact the degree of blood flow restriction. The amount of tissue surrounding the blood vessels influences the pressure exerted on the vasculature and impact the degree of blood flow restriction (Hargens et al. 1987). Initial investigations by Loenneke et al. (2012) suggested pressures to restrict blood flow of the lower body should be determined by limb circumference, supported by findings that thigh circumference is the biggest predictor of arterial occlusion in the lower body ( $\beta=0.570)$ (Loenneke et al. 2015). This reinforces that pressures in the lower body should be 
individualised according to thigh size, meaning bigger thighs required greater pressures, consistent for both sexes. However, this research also identified brachial systolic blood pressure may be an additional significant predictor $(\beta=0.231)$ (Loenneke et al. 2015) (see section 2.4.1), consequently this approach was adopted in the current study. However, our study found no improvement in recovery rate which contrasts findings of Beaven et al. (2012) who identified improved recovery of peak power, $24 \mathrm{~h}$ after occlusion relative to a control trial. Although comparable durations of vascular occlusion were used (i.e. two cycles of three min occlusion and three min reperfusion), a significant contrast was cuff pressure in which a standardised $220 \mathrm{mmHg}$ was applied. However, since Northey et al. (2016) replicated the occlusion protocol of Beaven et al. (2012) with standardised cuff pressures, and found no improvement in recovery, conclusions cannot be drawn regarding optimal cuff pressure for recovery protocol. Accordingly, alternative methodological factors must be influencing the resulting outcome of using vascular occlusion as a recovery modality. For instance, cuff pressure was determined from a regression equation (Loenneke et al. 2015) based on non-elite participants, although this protocol considers thigh girth, tissue type (muscle or fat) may impact the level of blood flow restriction, a variable which will be different between elite trained and untrained participants.

An alternative and large methodological discrepancy existing between studies using occlusion for recovery is the training status of the participants. Research completed by Beaven et al. (2012) investigated healthy, active non-sport participating males and found vascular occlusion improved recovery, similar in Page et al. (2017). However, research completed by Northey et al. (2016) and within the current study investigated the use of vascular occlusion in well trained individuals, identifying no effect on recovery. This potentially suggests training status may impact upon the success of vascular occlusion as a recovery strategy and speculatively is supported by the difference in CK values reported in the current study at $24 \mathrm{~h}$ (Occlusion: $409 \mu \cdot \mathrm{L}^{-1}$; Con: 506 $\mu \cdot \mathrm{L}^{-1}$ ) in contrast to results reported by Page et al. (2017) (Occlusion: $335 \mu \cdot \mathrm{L}^{-1}$; Con: $656 \mu \cdot \mathrm{L}^{-1}$ ). A review by Meneghel et al. (2013) indicated there is a reduction in the magnitude of indirect markers of muscle damage when the trained populations conduct repeated exercise sessions compared to untrained and/or sedentary participants (Chen et al. 2006) and Falvo et al. (2009), reported the magnitude of changes is small without significant differences when trained individuals are investigated. Newton et al. (2008) supports this when comparing untrained to resistance trained men completing 6 sets of maximal eccentric exercise of 6 repetitions elbow flexors at constant speed. Maximal voluntary contraction, range of motion, plasma CK and delayed onset of muscle soreness were measured before, immediately and 5 days after exercise. The trained group showed significantly $(\mathrm{p}<0.05)$ smaller changes in the measured variables and faster recovery of muscle function when compared to the untrained group. Even with a group of active or well-trained 
participants, high inter-individual variability in EIMD marker responses has been found even when participants perform the same exercise (Damas et al. 2016). Research suggests that the large interindividual variability including non-modifiable factors (ethnicity, age, and gender) could be responsible for most of the equivocal findings and uncertainties regarding EIMD etiology (Damas et al. 2016); another variable potentially explaining variation between the current study and previous work investigating the use of vascular occlusion for recovery. Therefore, it may be important to examine the use of vascular occlusion on an individual basis as it may be a practical recovery option for some athletes/players and at some levels of performance.

A novel aspect of this research was the response of a physical stress test as an indicator of recovery status and readiness-to-train following sprint exercise at $24 \mathrm{~h}$. Within the literature, previous recovery studies have assessed response to a recovery strategy when the athlete is rested, however players are frequently required to return to training $24 \mathrm{~h}$ after a match. Therefore, hormonal response to a physical stress test may indicate if the players are adequately recovered and able to return to training. In the current study, $\mathrm{T}$ increased pre-to post stress test in both conditions (Recovery: $21.58 \pm 44.90 \mathrm{pg} \cdot \mathrm{ml}^{-1}, 7 \pm 17 \%$, p=0.031; Con: $10.62 \pm 36.57 \mathrm{pg} \cdot \mathrm{ml}^{-1}, 4 \pm 13 \%$, $\mathrm{p}=0.178$ ), but $\Delta \mathrm{T}$ was not significantly different between conditions, suggesting Recovery in comparison to Con had no impact on hormonal response. Furthermore, $\mathrm{C}$ showed a significant decline from pre-to post-stress test in both the Recovery $\left(0.14 \pm 0.23 \mu \mathrm{g} \cdot \mathrm{dl}^{-1}, 38 \pm 72 \%, \mathrm{p}=0.007\right)$ and Con $\left(0.17 \pm 0.27 \mu \mathrm{g} \cdot \mathrm{dl}^{-1}, 50 \pm 94 \%, \mathrm{p}=0.006\right)$. Therefore, within the current study, consistent with rested results, there was no difference between conditions regarding rate of recovery following a physical stress test.

Limitations of the research should be acknowledged; the placebo effect is not accounted for, as also specified in Chapter 5, which may be an important factor as previous research has highlighted the ability of the placebo effect to improve performance (Cook \& Beaven 2013). Within Con, cuffs were still applied which was still novel for the players in comparison to lying and resting with the absence of cuffs and it is difficult to blind participants from the conditions due to the obvious difference in cuff pressure. Therefore, a further condition would be optimal to identify the impact of vascular occlusion, determining whether there is a placebo or physiological effect on performance when compared against a control in which cuffs are absent as completed by Marocolo et al. (2015). Similarly, it is difficult to blind testers to conditions due to variation in cuff pressure, however, all testers and coaches were informed that no verbal encouragement was to be given during exercise (sprints, jumps, stress test). An additional limitation lies with thigh girth measurements which were taken pre-sprints to reduce time from completion of sprints to applying occlusion cuffs (as would be completed within a practical setting). However, previous research has reported the impact of exercise on interstitial fluid; plasma volume may fall $\sim 20 \%$ with intense 
exercise over 15 min due to fluid movement towards active muscle (Astrand et al. 1986). Alterations in the osmolality of skeletal myocyte cytoplasm and interstitial fluid due to local release of metabolic factors from skeletal muscle increase solutes in the interstitial fluid (Astrand et al. 1986). Also, an increase perfusion of capillary beds in active muscles increase capillary hydrostatic pressure, which drives more fluid from the plasma to the interstitum (Brooks et al. 2005). Therefore, thigh girth may be affected and should be measured post-sprint, immediately prior to cuff placement to determine inflation pressure, this will ensure pressure is correctly applied for each individual.

\subsection{PRACTICAL IMPLICATIONS}

The current research offers practical application to coaches and performance scientists, despite vascular occlusion not influencing recovery time at rest or increasing readiness-to-train after $24 \mathrm{~h}$. Physiological and performance markers were consistent across both conditions and thus alleviates any concerns around using this ergogenic strategy within season as there was no negative impact. It would be suggested to test individual response, considering previous research highlighting the importance of individual perception of recovery strategies on subsequent performance (Cook \& Beaven 2013) to identify any beneficial effects for individuals considering the ease of use, low cost and minimal time requirement. This is further emphasised as variables such as EIMD protocol, cuff pressure, duration of occlusion and training status may impact the effectiveness of vascular occlusion as a recovery strategy and therefore, should be tested on an individual basis to determine if vascular occlusion may be an effective recovery strategy to implement.

\subsection{CONCLUSION}

In conclusion, the data presented in this investigation suggest that the application of two cycles of three min intermittent vascular occlusion administered following maximal sprint exercise did not improve recovery but was also not detrimental to physiological or neuromuscular markers of recovery at $2 \mathrm{~h}$ or $24 \mathrm{~h}$ following rest or after a physical stress test. 


\section{Chapter 7 - Synthesis of findings}

\subsection{SYNTHESIS OF FINDINGS}

In the following synthesis the main findings from the three experimental chapters and their practical application to coaches and performance scientists will be discussed in relation to the main themes of this thesis, acknowledging limitations, practical implications and future research prior to providing final conclusions. The series of studies comprising this thesis have endeavoured to add to the existing body of literature concerning the use of vascular occlusion and limb IPC to improve elite sport performance and contributing information to performance scientists to inform applied practice.

Pre-competition and recovery are two areas, in addition to training and competition, that performance scientists can implement to improve athletic performance. Vascular occlusion/limb IPC are emerging strategies, providing the overriding theme of this thesis, implemented as a strategy to improve athletic performance through trying to optimise preparation and recovery. Vascular occlusion/limb IPC has been previously defined as application of cuffs to the proximal limbs, inflated and deflated either during exercise and rest periods respectively or for cycles of ischemia and reperfusion whilst lying at rest in the supine position. The external pressure aims to maintain partial arterial inflow to the muscle while occluding venous return (Pope et al. 2013). However, the lack of specific research in elite athletes, as identified in the review of literature, provided difficulties applying previous research as positive effects of these strategies examined in a healthy population may not transfer into performance improvements in elite athletes due to differences in physiological responses in comparison to un-trained participants (Marocolo et al. 2016). The majority of research had examined healthy participants in their samples, while this population are more accessible and overcomes difficulties of accessing elite athletes to complete research into vascular occlusion and limb IPC, it is difficult to transfer a positive effect seen with vascular occlusion/limb IPC to elite populations due to differences in physiological responses as a result of training (Marocolo et al. 2016). For example, statistically significant differences have been observed in systolic blood pressure and HR both before and after exercise $(\mathrm{p}<0.001)$ between trained and untrained males. Trained athletes are able to increase their systolic blood pressure with relatively lower HR as compared to untrained individuals (Vandavasi \& Sukumar 2016). Also, unlike untrained individuals, elite athletes present only a small window for adaptation or improvement in performance, requiring a higher dose to generate a response in highly trained athletes to improve performance (Cormie et al. 2011). Therefore, this thesis completed three investigations into the use of vascular occlusion/limb IPC with elite athletes to contribute to the body of literature and offering insight for performance scientists for practical application. Data 
collection was successfully completed with elite athletes in all experimental chapters as evidenced by competition standards, however, this inherently limited sample size and posed significant timepressures which precluded the addition of a third experimental condition, for instance, in Chapter 4 examining the effect of morning exercise without vascular occlusion to replicate previous research in athletes would have improved conclusions and advice for performance scientists, however this was restricted by coaches and athletes.

Alternative methodological limitations have previously impacted application in sport, specifically relating to research not individualising cuff pressure which is personally deemed important as within elite sport it is essential to individualise strategies. Despite previous research acknowledging the same pressure may not restrict blood flow similarly in each individual, related to tissue surrounding the blood vessels. Previous studies had implemented an occlusion protocol prescribing an arbitrary restrictive pressure for all participants or based on systolic blood pressure at rest (Fatela et al. 2016). Recent findings however indicate that neither one of these approaches represented an effective strategy for controlling the magnitude of blood flow restriction in the lower body (Loenneke et al. 2012). If restrictive pressures are too low, muscular responses may not be significantly augmented (Scott et al. 2015). Furthermore, extremely high pressures (i.e. those that occlude arterial inflow during inter-set rest and/or exercise) may not enhance muscular development more than moderate pressures (Scott et al. 2015). It is accepted that vascular occlusion pressure should be high enough to occlude venous return from the muscles, yet low enough to maintain arterial inflow into the muscle (Loenneke et al. 2014). Logic therefore dictates that vascular occlusion should not be universally applied at an absolute pressure, but should vary relative to each individual (Loenneke et al. 2013). Therefore, to ensure practical application for elite athletes, both male and females, this was an important area investigated in this thesis. The decision to individualise cuff pressure was based on Loenneke et al. (2015) regression model ensuring factors of blood pressure and thigh girth were considered, which as tissue surrounding the vessel may impact occlusion pressure provides increased precision in comparison to blood pressure alone. Furthermore, it offers a practical method to use in the field in comparison to Doppler measurements which would be difficult in an applied setting for performance scientists to complete with a squad of swimmers or rugby team, for instance. The ranges, $147-266 \mathrm{mmHg}$, across the experimental chapters highlight the variance between individuals and requirement for the protocol to acknowledge that a standard occlusion pressure may not reduce blood flow to the same degree in different individuals.

As chapter 2 highlighted, cuff width can also impact on occlusion pressure, the same cuffs were utilised for all studies, enabling consistent application of occlusion pressure to the thighs in all experimental chapters. Wider cuffs $(11 \mathrm{~cm})$ were applied following research suggesting more 
effective restricting of arterial blood flow at lower inflations (Crenshaw et al. 1988) and recommendations by Wernbom et al. (2006). While an individual approach was adopted, no performance improvements were identified as a result of this strategy. This may either be linked to the training status and small window for improvement as discussed. However, individualisation was based upon Loenneke et al. (2015) which determined factors that should be accounted for when setting cuff pressure or level of blood flow restriction, but completed in non-elite male and female population (reported absence of cardiovascular or metabolic disease as criteria). Although this protocol considers thigh girth, tissue type (muscle or fat) may impact on the level of blood flow restriction, a variable which will be different between elite trained and untrained participants. Hunt et al. (2016) have recently reported the degree of intramuscular pressure depends on the architecture features of the muscles; hypertrophied muscles have a greater thickness and volume, subsequently blood flow occlusion may occur at relatively lower pressures (Wernbom et al. 2006). Fat as opposed to muscle cross sectional area have explained most of the variance in the cuff pressure required to completely occlude arterial flow (Loenneke et al. 2011). Despite attempts to individualise the occlusion pressures implemented in this study (Loenneke et al. 2015), there may have been between-participant variation in the degree of occlusion experienced during vascular occlusion. Variance in research on the impact of tissue composition needs to be addressed and research completed in trained compared to untrained participants to determine differences in occlusion pressures, if required. Further research into individual factors modulating the degree of pressure experienced by participants in vascular occlusion will likely better inform the use of this ergogenic modality in an athletic environment for coaches, athletes and performance scientists.

The decision for the focus on practical application for elite populations and use of vascular occlusion/limb IPC determined three specific research questions, implementing individualised cuff pressure across a range of individual and team sports. One of the important considerations for all three experimental studies was to ensure that all interventions could feasibly be implemented into real-world competition settings. Therefore these were established in accordance to the models proposed by Jones et al. (2017) and Coutts (2016) in which models have been proposed to undertake and integrate research within practice:

i. Investigate the use of vascular occlusion combined with resistance exercise in the morning ( $5 \mathrm{~h}$ prior) as a priming strategy to improve afternoon strength and power performance including identifying hormone responses and differences in responses between sexes (Chapter 4)

ii. Investigate the use of limb IPC applied in the early $(2 \mathrm{~h})$ and late phase $(24 \mathrm{~h})$ on swimming performance (Chapter 5) 
iii. Investigate the use of vascular occlusion as a recovery strategy following sprint exercise in team sports (Chapter 6)

When considering findings of Chapter 4, AM exercise with vascular occlusion had no statistically significant effect on PM performance, but a decline in salivary $\mathrm{T}$ concentration over the $5 \mathrm{~h}$ period was attenuated (Con: $-23.6 \pm 19.9 \%$; RE+VO: $-5.8 \pm 22.1 \%$ ), with significant increases in $\mathrm{T}$ post exercise $(10.7 \pm 21.5 \%)$. Regarding acute $\mathrm{T}$ response, previous research has discussed the requirement of high intensity and volume thresholds to be reached in order to achieve a hormone (T) response with examples from Ratamess et al. (2005) suggesting 6x10 repetitions but not 1x10 resulted in a $\mathrm{T}$ response. This has been linked to the metabolic accumulation required to induce a $\mathrm{T}$ response. Consequently, previous research implementing morning exercise to influence afternoon $\mathrm{T}$ and performance have implemented high volumes of resistance exercise (e.g. $5 \times 10$ bench press; Russell et al 2015), however, this lacks practicality for completion the day of competition especially for particular sports such as weightlifting. The use of combining resistance exercise and vascular occlusion was hypothesized to attentuate the circadian decline in $\mathrm{T}$, due to mechanisms associated with increased metabolic accumulation, the result of decreased venous outflow and consequently increasing hormone response with a lower volume and intensity of resistance exercise, which was achieved. As maximal exercise elicits a rapid endocrine response that normally relates to exercise intensity (Buśko \& Opaszowski 2005), and correlations between blood lactate and T changes exist following heavy resistance exercise (Kraemer et al. 1991), a direct stimulatory effect of lactate may lead to increased release of T (Lu et al. 1997). Notably, the $78.9 \pm 13.6 \%$ increase in blood lactate concentrations that occurred immediately after RE+VO exposure, when the whole group was analysed, correlated positively $(\mathrm{r}=0.559)$ with $\Delta \mathrm{T}$ concentrations in chapter 4. Such findings corroborate the use of vascular occlusion combined with resistance exercise to be used as a method to immediately increase and also attenuate the decline in $\mathrm{T}$ from $\mathrm{AM}$ to PM in elite athletes.

A novel aspect of this study investigated the female response to AM exercise and hormonal priming. A large portion of research into the effect of $\mathrm{T}$ concentrations in response to exercise has been completed in males as discussed in Chapter 2 and 4 in which it was highlighted results are not consistent between males and females. Within the current thesis, female $\mathrm{T}$ response followed a similar pattern to males and combined group results; a decrease of $17.2 \pm 21.1 \%$ was displayed in the control from AM to PM whereas morning exercise attenuated this decline to $1.6 \pm 25.5 \%$ within RE+VO. Similarly, females also showed an acute increase in T response $(8.3 \pm 17.5 \%)$ to exercise with vascular occlusion, which is comparable to the increase seen in male participants $(13.6 \pm 26.3 \%)$. The aforementioned studies investigated females that were active, however research complete by Cook et al. (2012) identified baseline measures of T were significantly higher 
at weekly time points over 12 weeks in elite (international level athletes) $\left(87 \mathrm{pg} \cdot \mathrm{ml}^{-1}\right)$ than nonelite (national level athletes) $\left(41 \mathrm{pg} \cdot \mathrm{ml}^{-1}\right)$ female athletes. This may support the results of this study as the average baseline for females involved was $97.92 \pm 24.46 \mathrm{pg} \cdot \mathrm{ml}^{-1}$. The results highlight a similar circadian decline from AM to PM, and the use of morning exercise can attenuate this decline in $\mathrm{T}$ in elite female athletes.

Despite RE+VO reducing the circadian decline in T, across all groups, no PM performance effects were observed for CMJ or IMTP; findings which contradict previous work (Russell et al. 2015; Cook et al. 2014). Results were similar in performance change between all groups, except the improvement in IMTP peak force was greater in weightlifting only athletes $(3.2 \pm 7.7 \%)$. Previous research has suggested baseline strength and power may determine responsive properties, for example, a significant effect of baseline strength on the relationship between $\mathrm{T}$ and squat performance has been observed; $T$ correlated to squat performance $(r=0.92)$ in strong participants compared to weaker counterparts $(r=0.35)$ (Crewther et al. 2012). The expression of force and power may be dependent on individual variation in salivary free $\mathrm{T}$ concentration and/or existing strength and power levels (Crewther et al. 2012) which may support that weightlifting athletes have increased baseline strength and power compared to field athletics and thus this group alone showed (although not significant) a greater improved PM performance in a test specific to their competition performance. The relationship between baseline strength and responsive properties of T may also explain the individual variation observed in results, especially of the IMTP in which 13 out of 22 subjects improved peak force (2 to $17 \%$ ).

Alternatively, individual variation in factors associated with the efficacy of RE+VO, such as the balance between muscle activation and fatigue (contractile/metabolic impairment), may have contributed to such findings. Vascular occlusion with resistance exercise implicates greater muscular activation to maintain the same total work output (Takarada et al. 2000; Takarada et al. 2002; Yasuda et al. 2008; Fahs et al. 2015) and additional motor unit activity and enhanced recruitment of type II motor units (Moore et al. 2004) is required to maintain force levels following resistance exercise combined with vascular occlusion (Fatela et al. 2016). Also the level of vascular occlusion will influence hemodynamic signals, $\mathrm{O}_{2}$ and nutrient delivery and the accumulation and clearance rate of local metabolic by-products during exercise (Takarada et al. 2000; Karabulut et al. 2011) therefore ensuring an equal and appropriate level of vascular occlusion between participants is important to achieve consistent gains. Implementation of Doppler ultrasound measurements would increase control over the level of vascular occlusion and ensure specificity for each individual but may not be practical to use within the field. Despite our attempts to individualise occlusion pressures there may have been between-participant variation in the degree of occlusion, muscle activation and fatigue experienced. Therefore, further research is 
required to identify individual factors that contribute to the optimisation of resistance exercise with vascular occlusion for use prior to subsequent exercise whether implemented immediately or $5 \mathrm{~h}$ before. At present, performance scientists should trial this individually with athletes, consequently, response will be directly applicable to individual athletes and should be practiced ensuring tolerance of vascular occlusion to enhance performance, adjustment of volume and intensity for individuals can therefore be completed as required to ensure the balance between muscle activation (and hormone response) and fatigue are achieved.

Despite providing a novel insight into the use of AM resistance exercise combined with vascular occlusion on PM performance in elite athletes, several experimental limitations are acknowledged. The study design limits conclusions which can be drawn from the results; a traditional experimental study design was completed; however, this inherently prevents identification of the combined effect of resistance exercise and vascular occlusion attenuating the decline in T. Only from previous research recommending the requirement for volume/intensity threshold to be achieved to increase $\mathrm{T}$, and previous morning exercise results, can speculations be made; however, it cannot be inferred if occlusion was responsible for the $\mathrm{T}$ response. The addition of a nonoccluded AM resistance exercise-only trial would have allowed further delineation of the effects of AM resistance exercise with vascular occlusion on physiological and performance responses. This study did not identify short term performance response following vascular occlusion and resistance exercise as the focus was to look at exercise priming, due to increased practical application for the sports and athletes involved. However, further application may identify immediate performance response, considering previously reported increased muscle activation with this strategy (Fatela et al. 2016). Further investigation of muscle activation in response to exercise with vascular occlusion, in elite athletes, may inform potential application of this strategy to be performed in sport where opportunity exists to complete immediately prior to performance. Finally, it would have been relevant due to the focus on practical application to determine smallest worthwhile enhancement in performance. Ideally it would have been preferred for participants to complete CMJ and IMTP due to reliability of measures followed by performance measures specific to competition i.e. weightlifting to complete maximal snatch. However, due to the phase of training coaches and athletes were reluctant to lift $100 \%$ of maximal effort both due to risk of injury and psychological impact. Changes in performance between control and RE+VO could have been contrasted to the percentage differences between competitions to determine if the improvement was greater than the variation observed and consequently deemed if a worthwhile enhancement was achieved according to Hopkins et al. (1999).

Findings of Chapter 5 suggest swimming performance was not improved by limb IPC when applied $2 \mathrm{~h}$ or $24 \mathrm{~h}$ prior to performance. Additionally, no influence either positive or negative 
was observed on physiological markers examined. The results at $2 \mathrm{~h}$ oppose previous research that found limb IPC acutely improved swimming performance (Jean-St-Michel et al. 2011; Marocolo et al. 2015; Lisbôa et al. 2017). However, consistent with previous research (Seeger et al. 2017), no change in combined swimming performance was observed when limb IPC was applied $24 \mathrm{~h}$ prior to the time trial $(+0.2 \pm 1.4 \%)$. Furthermore, the negative effect that was observed in the $200 \mathrm{~m}$ following both conditions compared to a control contrasted previous results. However, it should be considered only 5 participants completed $200 \mathrm{~m}$, one of which decreased performance by $4 \%$, negatively skewing results. This large variation in performance is unusual for elite athletes, supported by research completed by Pyne et al. (2004) in which variation in performance is reported to be $0.8 \%$ between competitions. Therefore, additional unknown variables may be influencing individual performance observed. Calculation of smallest worthwhile change revealed 9 participants had a smallest worthwhile change in performance following the use of IPC-2h, with variation in change required ranging from $0.00-0.29 \%$. Whereas 11 participants showed a smallest important change after completion of IPC-24h with a required change ranging from $0.01-0.45 \%$ when results were combined as a group. Within the current results the individual variation in performance, although speculative may be consequence of different training backgrounds in which some athletes training has focussed more aerobically or anaerobically affecting energy system contribution during TT performance and the impact of limb IPC, or potential impact of the differences in stroke technique, however no patterns have been identified within the current results providing support of this.

Alternatively to individual responses to limb IPC, disparity in results might be explained by considering location of the cuff in comparison to previous research. Within this thesis, cuffs were applied to the thighs following evidence of a systemic change in blood flow. However, no differences between conditions were identified regarding physiological measures. Alternative research has suggested limb IPC may cause local changes in the muscle at the site of the cuff (Horiuchi et al. 2015; Kjeld et al. 2014). As discussed in Chapter 5, in swimming, the contribution of propulsive force is approximately 90\% for the upper extremities (Deschodt et al. 1999; Morouço et al. 2011) therefore the local changes achieved by application of the cuffs to the upper limbs may increase effectiveness of limb IPC to improve swimming performance rather than a systemic response due to the large emphasis required on the upper body.

To date, only one study had examined the use of limb IPC $24 \mathrm{~h}$ prior to performance to identify the impact of the late phase on subsequent performance. Results of the current study were consistent with this, however, acknowledging previous methodological limitations of these findings, application of cuffs on the thighs rather than upper body may have affected these results. Future research is recommended to apply cuffs to the upper body and implement limb IPC $24 \mathrm{~h}$ 
prior to performance due to the practical application of this strategy in elite sport. Additional physiological measures may have delineated the results to determine if limb IPC affected endothelial dysfunction (through measurement of FMD), as one of the predominant mechanisms influencing performance. Limb IPC has shown to prevent endothelium dysfunction (Dawson et al. 2013; Bailey et al. 2012), increasing aerobic contribution to performance and giving a greater understanding to enable application. For instance, both FMD and NIRS have previously been investigated as measures to examine physiological responses associated with limb IPC (outlined in section 2.5.4). Practical implications of measuring FMD and NIRS in a swimming environment provided difficulty, but furthermore, due to working with elite athletes, although a strength and focus of this thesis, restricted access and time available to complete the investigations. Both athletes and coaches requested limited disruption and minimal time distracted from training; this was achieved but inherently limited time for data collection.

Practically, the results provide baseline data for use of limb IPC in swimming when investigated with well-trained to elite athletes. In comparison to previous research, this study identifies swimming performance may only benefit when cuffs are applied to the upper body to achieve local adaptations rather than on the thighs where a systemic response would be anticipated. Limitations of the research were acknowledged; the placebo effect is not accounted for, which may be an important factor as previous research has highlighted the ability of the placebo effect to improve performance (Cook \& Beaven 2013). A further condition would be optimal to identify the impact of vascular occlusion, determining whether there is a placebo or physiological effect on performance when compared against a control in which cuffs are absent. While separate analyses of the male and female results would have been preferable, the limited number of swimmers available within the experimental investigation did not permit this.

Of interest, both chapter 4 and 5 included male and female participants, whereas the majority of previous research in IPC and sport performance has focussed on male only participants, with the exceptions of Gibson et al. (2013) who identified no improvement in performance in males and females and Paradis-Deschênes et al. (2017) which is the only study to really investigate if differences in gender exist between the effect of IPC on sport performance. Within clinical literature, Abete et al. (2011) reported IPC is effective for younger but not aged hearts, supported by further research proposing that post ischemic functional recovery and infarct size (in rabbits) are affected by age but not by gender (McCully et al. 2006), providing initial suggestions that IPC may be more effective in some populations than others. However, it has also been noted that females appear to display smaller clinical benefits compared with male counterparts. In a cohort of 382 participants, failure to induce preconditioning effects during percutaneous coronary intervention was observed in females (Laskey \& Beach 2003), speculated to be due to the innate, multiple, protective actions of estrogen (Pitcher et al. 2005). Additionally, in a sporting context, 
females have been reported to exhibit less fatigue than males during intense exercise (Parker et al. 2007; Hunter 2016) but not under conditions of ischemia (Russ \& Kent-Braun 2003) suggesting that muscle perfusion and oxygenation may be involved in sex-related differences. Females exhibit greater vasodilation in the limbs during single knee extensions (Parker et al. 2007) and greater capacity for utilising oxidative metabolism than males (Kent-Braun et al. 2002). Thus, females exhibit a greater reliance on oxidative metabolism compared with males, which could challenge the ergogenic impact of IPC (Paradis-Deschênes et al. 2017). This is indeed supported by the findings of Paradis-Deschênes et al. (2017) in which limb IPC improved physical performance in strength-trained males through improved $\mathrm{O}_{2}$ delivery and utilisation in comparison to female counterparts during repeated, maximal efforts of knee extension exercise. Results showed IPC increased total muscular force in males only (13.0\%) and this change was greater than in females (10.4\% difference). IPC also induced different changes between sexes for average muscle $\mathrm{O}_{2}$ uptake, for example set 2: males $6.4 \%$; females $-16.7 \%$ and set 5: males $10.2 \%$; females $-40.4 \%$, despite similar increases in muscle blood volume at rest and during recovery between sets of both sexes. The initial IPC-induced increase in $\mathrm{O}_{2}$ delivery was also similar between sexes.

The difference in response between sexes does not explain results of chapter 4 as no differences in performance were observed when males and females were analysed separately. However, this may be explained by a single effort being performed in comparison to the repeated maximal efforts described in the study by Paradis-Deschênes et al. (2017) or consequence of different mechanisms underpinning performance when vascular occlusion is combined with exercise. Opposing this, the difference in response between genders may explain some of the individual variations observed in chapter 5. As highlighted, the split in male/female participants did not allow for separate analyses to be completed, however within swimming a decrease in $\mathrm{O}_{2}$ delivery could be detrimental to performance. The physiological mechanisms required for performance of five sets of five maximal knee extensions will contrast to 100 and $200 \mathrm{~m}$ swimming and therefore results cannot be extracted from the study and applied to the current results. However, this does highlight an important area for future research, identifying the difference in physiological response and performance between sexes if limb IPC is being considered for application in elite sport by performance scientists.

Chapter 6 investigated the use of vascular occlusion as a recovery strategy in contrast to the previous two chapters examining its use as a pre-competition strategy. Vascular occlusion applied as a recovery strategy did not influence any physiological or neuromuscular markers measured in the $2 \mathrm{~h}$ or $24 \mathrm{~h}$ after sprint exercise in professional academy rugby union players. Moreover, no significant differences existed in hormone response to a physical stress test at $24 \mathrm{~h}$, between conditions. However, it is notable there was no detrimental effect on physiological or neuromuscular markers assessed, alleviating any concerns of impairment when using this strategy. The current study replicated the study design by Beaven et al. (2012), however our results oppose 
those previously identified, where improved recovery of peak power, $24 \mathrm{~h}$ after occlusion (relative to a control) was observed. Individualisation of cuff pressure was one methodological difference which may explain variability in results. However as stated in Chapter 6, Northey et al. (2016) replicated the study by Beaven et al. (2012), including cuff pressure, and also found contrasting results. Thus, training status of participants offered further explanation as discussed in chapter 6; variation in CK response between the current study and Page et al. (2017). A review by Meneghel et al. (2013) indicated there is a reduction in the magnitude of indirect markers of muscle damage when the trained populations conduct repeated exercise sessions compared to untrained and/or sedentary participants (Chen et al. 2006). Newton et al. (2008) supports this, the trained group showed significantly $(p<0.05)$ smaller changes in the measured variables (MVC, CK and delayed onset muscle soreness) and faster recovery of muscle function when compared to the untrained group. Research by Beaven et al. (2012) investigated healthy, non-sport participating males and found vascular occlusion improved recovery. However, the current study, with focus on elite athletes, and Northey et al. (2016) both investigated the use of vascular occlusion for recovery in well trained athletes, suggesting training status may impact upon the success of this strategy.

A novel approach to assess recovery following EIMD involved assessment of hormone response following the completion of a physical stress test to provide an indicator of readiness-to-train. Again, acknowledging the practical application for elite sport as a focus of this thesis, players are frequently required to return to training $24 \mathrm{~h}$ after a match. Consequently, hormonal response to a physical stress test may indicate if players are adequately recovered and can return to training following research suggesting $\mathrm{T}$ is a stress biomarker and identified relationships between $\mathrm{T}$ concentration in response to a mid-week stress test and match outcome (Crewther et al. 2017). However, $\mathrm{T}$ increased pre-to post stress test in both conditions (Recovery: $21.58 \pm 44.90 \mathrm{pg} \cdot \mathrm{ml}^{-1}$, $7 \pm 17 \%, \mathrm{p}=0.031$; Con: $\left.10.62 \pm 36.57 \mathrm{pg} \cdot \mathrm{ml}^{-1}, 4 \pm 13 \%, \mathrm{p}=0.178\right)$, but $\Delta \mathrm{T}$ was not significantly different between conditions, suggesting Recovery in comparison to Con had no impact on hormonal response. Further research is required into the application of vascular occlusion as a recovery strategy for elite athletes, determination of an optimal protocol is required. However, performance scientists can be confident there are no detrimental effects on physiological and neuromuscular markers and can therefore be trialled with individuals to examine impact on recovery and subsequent performance. Further research is required on the use of hormone response to a physical stress test as an indicator of recovery status and readiness to train.

\subsection{FUTURE RESEARCH}

Despite no improvements identified in athletic performance, the experimental chapters highlight key areas for future research to enable application in elite sport of vascular occlusion/limb IPC: 
- Determination of cuff pressure in elite athletes, determining an optimal protocol with associated physiological and performance responses

- Application of vascular occlusion with resistance exercise to increase muscle activation applied immediately prior to athletic performance

- Replicate chapter 5, applying cuffs to the upper body to examine the late phase (24 h) of IPC on swimming performance

- Determine a protocol for recovery regarding optimal cycles and length of occlusion

- Determine physiological mechanisms causing variation in response between individuals to explain differences in performance

- Further investigate the use of a physical stress test and hormone response as an indicator of readiness-to-train and success of a recovery strategy

\subsection{SUMMARY OF PRACTICAL IMPLICATIONS}

Summary of practical implications from experimental chapters:

- Performance scientists, coaches and athletes can be confident vascular occlusion/IPC has no detrimental effect on physiological or performance measures when used as a precompetition or recovery strategy

- Vascular occlusion combined with resistance exercise can increase T concentrations post exercise and reduce the decline over a $5 \mathrm{~h}$ period, found in both male and female athletes

- Tolerance of vascular occlusion with resistance exercise should be trialled to optimise the balance between activation and fatigue in individuals

- Variables such as cuff pressure, duration of occlusion and training status may be impacting effectiveness of vascular occlusion as a pre-competition or recovery strategy

- Performance Scientists should be aware of the current limitations determining cuff pressure and individual responses associated with pre-competition strategies, individually monitoring athletes could identify those that have a positive response to using these strategies to implement as part of competition preparation or recovery. 


\subsection{CONCLUSION}

In conclusion, this thesis has successfully examined the use of vascular occlusion/limb IPC as a pre-competition and recovery strategy within elite athletes. It is difficult to generalise and apply results from a healthy, untrained population into elite sport and therefore this thesis has discovered elite athletes' response to vascular occlusion combined with resistance exercise, limb IPC and vascular occlusion for recovery. Despite no performance improvements identified, more importantly this thesis has addressed some of the practical limitations of applying pre-determined protocols in sport and offered alternative protocols to be applied across a range of sports (strength/power, anaerobic, team sports) in areas which have not previously been investigated and provide clear practical implications and future research required to inform coaches and performance scientists how to effectively implement this ergogenic strategy to improve subsequent athletic performance, some of which are already being investigated and applied within the Welsh Institute of sport. 


\section{Bibliography}

Aardal, E. \& Holm, A.C., 1995. Cortisol in Saliva - Reference Ranges and Relation to Cortisol in Serum. Clinical Chemistry and Laboratory Medicine, 33(12), pp.927-932.

Abe, T. et al. 2005. Eight days KAATSU-resistance training improved sprint but not jump performance in collegiate male track and field athletes. International Journal of KAATSU Training Research, 1, pp.19-23.

Abe, T. et al. 2005. Skeletal muscle size and circulating IGF-1 are increased after two weeks of twice daily kaatsu resistance training. International Journal of KAATSU Training Research, 1(1), pp.6-12.

Abe, T., Kearns, C.F. \& Sato, Y., 2006. Muscle size and strength are increased following walk training with restricted venous blood flow from the leg muscle, Kaatsu-walk training. Journal of Applied Physiology, 100(5), pp.1460-1466.

Abete, P. et al., 2011. Ischemic preconditioning in the younger and aged heart. Aging Dis, 2(2), pp.138-148.

Adlercreutz, H. et al., 1986. Effect of Training on Plasma Anabolic and Catabolic Steroid Hormones and Their Response During Physical Exercise. J. Sports Med, 7, pp.27-28.

Allen, D.G., Lamb, G.D. \& Westerblad, H., 2008. Skeletal muscle fatigue: cellular mechanisms. Physiological Reviews, 88(1), pp.287-332.

Allison, R.C. \& Lawrence Bedsole, D., 2003. The Other Medical Causes of Rhabdomyolysis. The American Journal of the Medical Sciences, 326(2), pp.79-88.

Ammar, A. et al., 2014. Temporal specificity of training: intra-day effects on biochemical responses and Olympic-Weightlifting performances. Journal of Sports Sciences, 33(4), pp.358-68.

Anders, S.M. \& Watson, N. V, 2006. Short Report Menstrual Cycle Irregularities Are Associated With Testosterone Levels in Healthy Premenopausal Women. American Journal of Human Biology, 844(May), pp.841-844.

Andreas, M. et al., 2011. Effect of ischemic preconditioning in skeletal muscle measured by functional magnetic resonance imaging and spectroscopy: a randomized crossover trial. Journal of Cardiovascular Magnetic Resonance: Official Journal of the Society for Cardiovascular Magnetic Resonance, 13(1), pp.32.

Ardehali, H. \& O'Rourke, B., 2005. Mitochondrial K(ATP) channels in cell survival and death. Journal of Molecular and Cellular Cardiology, 39(1), pp.7-16.

Arregger, A.L. et al., 2007. Salivary testosterone: A reliable approach to the diagnosis of male hypogonadism. Clinical Endocrinology, 67(5), pp.656-662.

Bailey, S.J. et al., 2011. Fast-start strategy improves VO2 kinetics and high-intensity exercise 
performance. Medicine and Science in Sports and Exercise, 43(3), pp.457-467.

Bailey, T.G. et al., 2012. Effect of ischemic preconditioning on lactate accumulation and running performance. Medicine and Science in Sports and Exercise, 44(11), pp.2084-2089.

Bailey, T.G. et al., 2012. Remote ischemic preconditioning prevents reduction in brachial artery flow-mediated dilation after strenuous exercise. American Journal of Physiology. Heart and Circulatory Physiology, 303(5), pp.533-8.

Baird, M.F. et al., 2012. Creatine-kinase- and exercise-related muscle damage implications for muscle performance and recovery. Journal of Nutrition and Metabolism, 4, pp.1-13.

Balsalobre-Fernańdez, C., Tejero-Gonzaĺez, C.M. \& Del Campo-Vecino, J., 2014. Relationships between training load, salivary cortisol responses and performance during season training in middle and long distance runners. PLoS ONE, 9(8), pp. e106066.

Bangsbo, J., Mohr, M. \& Krustrup, P., 2006. Physical and metabolic demands of training and match-play in the elite football player. Journal of Sports Sciences, 24(7), pp.665-674.

Barbosa, T.C. et al., 2015. Remote ischemic preconditioning delays fatigue development during handgrip exercise. Scandinavian Journal of Medicine and Science in Sports, 25(3), pp.356364.

Barbosa, T.C. et al., 2014. Remote ischemic preconditioning delays fatigue development during handgrip exercise. Scandinavian Journal of Medicine \& Science in Sports, 25, pp.356-364.

Barcelos, L.C. et al., 2015. Low-load resistance training promotes muscular adaptation regardless of vascular occlusion, load, or volume. European Journal of Applied Physiology, 115, pp.1559-1568.

Barnett, A., 2006. Using recovery modalities between training sessions in elite athletes: Does it help? Sports Medicine, 36(9), pp.781-796.

Bateup, H.S. et al., 2002. Testosterone, cortisol, and women's competition. Evolution and Human Behavior, 23(3), pp.181-192.

Baxter, G.F., Goma, F.M. \& Yellon, D.M., 1997. Characterisation of the infarct-limiting effect of delayed preconditioning: Timecourse and dose-dependency studies in rabbit myocardium. Basic Research in Cardiology, 92(3), pp.159-167.

Beaven, C Martyn Gill, Nicholas D Cook, C.J., 2008. Salivary testosterone and cortisol responses in professional rugby players after four resistance exercise protocols. Journal of Strength \& Conditioning Research, 22(2), pp.426-432.

Beaven, C.M. et al., 2012. Intermittent lower-limb occlusion enhances recovery after strenuous exercise. Applied Physiology, Nutrition, and Metabolism, 37(6), pp.1132-9.

Beaven, C.M. et al., 2010. Ultradian rhythmicity and induced changes in salivary testosterone. European Journal of Applied Physiology, 110(2), pp.405-413.

Beaven, C.M., Cook, C.J. \& Gill, N.D., 2008. Significant Strength Gains Observed in Rugby Players after Specific Resistance Exercise Protocols Based on Individual Salivary 
Testosterone Responses. Journal of Strength and Conditioning Research, 22(2), pp.419-425.

Bessa, A. et al., 2016. Exercise intensity and recovery: Biomarkers of injury, inflamma- tion, and oxidative stress. Journal of Strength and Conditioning Research, 30(2), pp.311-319.

Bezerra de Morais, A.T. et al., 2017. Upper limbs total occlusion pressure assessment: Doppler ultrasound reproducibility and determination of predictive variables. Clinical Physiology and Functional Imaging, 37(4), pp.437-441.

Birnbaum, Y., Hale, S.L. \& Kloner, R.A., 1997. Ischemic Preconditioning at a Distance: Reduction of Myocardial Infarct Size by Partial Reduction of Blood Supply Combined With Rapid Stimulation of the Gastrocnemius Muscle in the Rabbit. Circulation, 96(5), pp.16411646.

Bishop, D., 2003. Warm up I: potential mechanisms and the effects of passive warm up on exercise performance. Sports Medicine, 33(6), pp.439-454.

Bishop, D., Bonetti, D. \& Dawson, B., 2001. The effect of three different warm-up intensities on kayak ergometer performance. Medicine and Science in Sports and Exercise, 33(6), pp.10261032 .

Bishop, D., Bonetti, D. \& Spencer, M., 2003. The effect of an intermittent, high-intensity warmup on supramaximal kayak ergometer performance. Journal of Sports Sciences, 21(1), pp.1320 .

Bishop, P.A., Jones, E. \& Woods, A.K., 2008a. Recovery from training: a brief review. Journal of Strength \& Conditioning Research, 22(3), pp.1015-1024.

Bishop, P.A., Jones, E. \& Woods, A.K., 2008b. Recovery from training: a brief review: brief review. Journal of Strength and Conditioning Research, 22(3), pp.1015-1024.

Bloomer, R., 2015. Considerations in the Measurement of Testosterone in Saliva and Serum Using ELISA Procedures. British Journal of Medicine and Medical Research, 5(1), pp.116-122.

Bolli, R. et al., 1997. Evidence that late preconditioning against myocardial stunning in conscious rabbits is triggered by the generation of nitric oxide. Circulation research, 81(1), pp.42-52.

Bolli, R., 2000. The late phase of preconditioning. Circ Res, 87(11), pp.972-983.

Bolli, R. et al., 1998. The nitric oxide hypothesis of late preconditioning. Basic Research in Cardiology, 93(5), pp.325-338.

Borne, R., Hausswirth, C. \& Bieuzen, F., 2016. Relationship Between Blood Flow and Performance Recovery: A Randomized, Placebo-Controlled Study. International Journal of Sports Physiology and Performance, (May), pp.1-44.

Bouma, M.G., van den Wildenberg, F.A. \& Buurman, W.A., 1997. The anti-inflammatory potential of adenosine in ischemia-reperfusion injury: established and putative beneficial actions of a retaliatory metabolite. Shock, 8(5), pp.313-20.

Brown, L.M. \& Hill, L., 1991. Some observations on variations in filament overlap in tetanized muscle fibres and fibres stretched during a tetanus, detected in the electron microscope after 
rapid fixation. Journal of Muscle Research and Cell Motility, 12(2), pp.171-182.

Buckingham, J.C., 2009. Glucocorticoids: exemplars of multi-tasking. British Journal of Pharmacology, 147(S1), pp.S258-S268.

Buckner, S.L. et al., 2017. Influence of cuff material on blood flow restriction stimulus in the upper body. Journal of Physiological Sciences, 67(1), pp.207-215.

Buijs, R.M. et al., 2003. The biological clock tunes the organs of the body: Timing by hormones and the autonomic nervous system. Journal of Endocrinology, 177(1), pp.17-26.

Bunevicius, K. et al., 2016. Cardiovascular response to bouts of exercise with blood flow restriction. Journal of Physical Therapy Science, 28(12).

Burckhartt, B. et al., 1994. Acadesine extends the window of protection afforded by ischaemic preconditioning in conscious rabbits. Cardiovascular Research, 28(9), pp.653-657.

Burnley, M. \& Jones, A.M., 2007. Oxygen uptake kinetics as a determinant of sports performance. European Journal of Sport Science, 7(2), pp.63-79.

Buśko, K. \& Opaszowski, B., 2005. Hormonal responses to repeated bouts of supra-maximal cycle ergometer exertions. Physical Education and Sport, 49(1), pp.8-12.

Byrd, S.K., 1992. Alterations in the sarcoplasmic reticulum: a possible link to exercise-induced muscle damage. Medicine and Science in Sports and Exercise, 24, pp.531-536.

Byrne, C., Twist, C. \& Eston, R., 2004a. Neuromuscular Function After Exercise-Induced Muscle Damage. Sports Medicine, 34(1), pp.49-69.

Byrne, C., Twist, C. \& Eston, R., 2004b. Neuromuscular function after exercise-induced muscle damage: theoretical and applied implications. Sports medicine (Auckland, N.Z.), 34(1), pp.49-69.

Cadore, E. et al., 2008. Correlations between serum and salivary hormonal concentrations in response to resistance exercise. Journal of Sports Sciences, 26(10), pp.1067-1072.

Calbet, J.A.L. et al., 2006. Effects of ATP-induced leg vasodilation on VO2 peak and leg O2 extraction during maximal exercise in humans. American Journal of Physiology: Regulatory, Integrative and Comparative Physiology, 291(2), pp.R447-53.

Cardinale, M. \& Stone, M.H., 2006. Is testosterone influencing explosive performance? Journal of Strength and Conditioning Research, 20(1), pp.103-7.

Cayot, T.E. et al., 2014. Effects of blood flow restriction duration on muscle activation and microvascular oxygenation during low-volume isometric exercise. Clinical Physiology and Functional Imaging, 36(4), pp.298-305.

Celec, P. et al., 2009. Salivary Sex Hormones during the Menstrual Cycle. Endocrine Journal, 56(3), pp.521-523.

Chan, R.K. et al., 2004. Reperfusion injury to skeletal muscle affects primarily type II muscle fibers. Journal of Surgical Research, 122(1), pp.54-60.

Chen, T.C., Nosaka, K. \& Sacco, P., 2006. Intensity of eccentric exercise, shift of optimum angle, 
and the magnitude of repeated-bout effect. Journal of Applied Physiology, 102(3), pp.992999.

Cheung, K., Hume, P.A. \& Maxwell, L., 2003. Delayed onset muscle soreness: Treatment strategies and performance factors. Sports Medicine, 33(2), pp.145-164.

Chiappin, S. et al., 2007. Saliva specimen: A new laboratory tool for diagnostic and basic investigation. Clinica Chimica Acta, 383(1-2), pp.30-40.

Chicharro, J.L. et al., 1998. Saliva composition and exercise. Sports Medicine, 26(1), pp.17-27.

Chtourou, H. et al., 2012. The effect of strength training at the same time of the day on the diurnal fluctuations of muscular anaerobic performances. Journal of Strength and Conditioning Research, 26, pp.217-25.

Clark, B.C. et al., 2011. Relative safety of 4 weeks of blood flow-restricted resistance exercise in young, healthy adults. Scandinavian Journal of Medicine and Science in Sports, 21(5), pp.653-662.

Clarkson, P.M., Nosaka, K. \& Braun, B., 1992. Muscle function after exercise-induced muscle damage and rapid adaptation. Medicine and Science in Sports and Exercise, 24(5), pp.512520 .

Claudino, J.G. et al., 2016. The countermovement jump to monitor neuromuscular status: A metaanalysis. Journal of Science and Medicine in Sport, pp.6-11.

Clevidence, M.W., Mowery, R.E. \& Kushnick, M.R., 2012. The effects of ischemic preconditioning on aerobic and anaerobic variables associated with submaximal cycling performance. European Journal of Applied Physiology, 112(10), pp.3649-3654.

Cochrane, D.J. et al., 2013. Does intermittent pneumatic leg compression enhance muscle recovery after strenuous eccentric exercise? International Journal of Sports Medicine, 34(11), pp.969974.

Cocking, S. et al., 2017. Is there an optimal ischaemic preconditioning dose to improve cycling performance? International Journal of Sports Physiology and Performance, 28, pp.1-25.

Coenen, C.M.H. et al., 1996. Changes in androgens during treatment with four low-dose contraceptives. Contraception, 53(3), pp.171-176.

Connolly, D.A.J., Brennan, K.M. \& Lauzon, C.D., 2003. Effects of active versus passive recovery on power output during repeated bouts of short term, high intensity exercise. Journal of Sports Science and Medicine, 2(2), pp.47-51.

Consitt, L. a, Copeland, J.L. \& Tremblay, M.S., 2001. Hormone responses to resistance vs. endurance exercise in premenopausal females. Canadian Journal of Applied Physiology, 26, pp.574-587.

Cook, C.J. Kilduff, L.P. Beaven, C.M., 2014. Improving strength and power in trained athletes with 3 weeks of occlusion training. International Journal of Sports Physiology and Performance, 9(1), pp.166-172. 
Cook, C.J. et al., 2014. Morning based strength training improves afternoon physical performance in rugby union players. Journal of Science and Medicine in Sport, 17(3), pp.317-321.

Cook, C.J. \& Beaven, C.M., 2013. Individual perception of recovery is related to subsequent sprint performance. British Journal of Sports Medicine, 47(11), pp.705-9.

Cook, C.J. \& Beaven, C.M., 2013. Salivary testosterone is related to self-selected training load in elite female athletes. Physiology \& Behavior, 116-117, pp.8-12.

Cook, C.J. \& Crewther, B.T., 2012. Changes in salivary testosterone concentrations and subsequent voluntary squat performance following the presentation of short video clips. Hormones and Behavior, 61(1), pp.17-22.

Cook, C.J., Crewther, B.T. \& Smith, A.A., 2012. Comparison of baseline free testosterone and cortisol concentrations between elite and non-elite female athletes. American Journal of Human Biology, 24(6), pp.856-858.

Cook, S.B., Clark, B.C. \& Ploutz-Snyder, L.L., 2007. Effects of exercise load and blood-flow restriction on skeletal muscle function. Medicine and Science in Sports and Exercise, 39(10), pp.1708-1713.

Cook, S.B., Murphy, B.G. \& Labarbera, K.E., 2013. Neuromuscular function after a bout of lowload blood flow-restricted exercise. Medicine and Science in Sports and Exercise, 45(1), pp.67-74.

Cooper, C.E. \& Brown, G.C., 2008. The inhibition of mitochondrial cytochrome oxidase by the gases carbon monoxide, nitric oxide, hydrogen cyanide and hydrogen sulfide: Chemical mechanism and physiological significance. Journal of Bioenergetics and Biomembranes, 40(5), pp.533-539.

Copeland, J.L., Consitt, L.A. \& Tremblay, M.S., 2002. Hormonal responses to endurance and resistance exercise in females aged 19-69 years. The Journals of Gerontology. Series A, Biological Sciences and Medical Sciences, 57(4), pp.B158-B165.

Cormie, P., McBride, J.M. \& McCaulley, G.O., 2009. Power-Time, Force-Time, and VelocityTime Curve Analysis of the Countermovement Jump: Impact of Training. Journal of Strength and Conditioning Research, 23(1), pp.177-186.

Cormie, P., McGuigan, M.R. \& Newton, R.U., 2011. Developing maximal neuromuscular power: Part 2 training considerations for improving maximal power production. Sports Medicine, 41(2), pp.125-146.

Coudene, P. et al., 2005. Evaluation of the ABX Pentra 400 : a newly available clinical. Clinical Chemistry and Laboratory Medicine, 43(7), pp.782-792.

Counts, B.R. et al., 2016. Influence of relative blood flow restriction pressure on muscle activation and muscle adaptation. Muscle and Nerve, 53(3), pp.438-445.

Coutts, A.J. et al., 2007. Monitoring for overreaching in rugby league players. European Journal of Applied Physiology, 99(3), pp.313-324. 
Coutts, A.J., 2016. Working fast and working slow: The benefits of embedding research in highperformance sport. International Journal of Sports Physiology and Performance, 11(1), pp.1-2.

Craig, A.B., 1986. Breath holding during the turn in competitive swimming. Medicine And Science In Sports And Exercise, 18(4), pp.402-7.

Credeur, D.P., Hollis, B.C. \& Welsch, M.A., 2010. Effects of handgrip training with venous restriction on brachial artery vasodilation. Medicine and Science in Sports and Exercise, 42(7), pp.1296-1302.

Crenshaw, A.G. et al., 1988. Wide tourniquet cuffs more effective at lower inflation pressures. Acta Orthopaedica Scandinavica, 59(4), pp.447-451.

Crewther, B.T. et al., 2012. Baseline strength can incluence the ability of salivary free testosterone to predict squat and sprinting performance. Journal of Strength and Conditioning Research, 26(1), pp.261-268.

Crewther, B.T. et al., 2017. Can salivary testosterone and cortisol reactivity to a mid-week stress test discriminate a match outcome during international rugby union competition? Journal of Science and Medicine in Sport.

Crewther, B.T. et al., 2015. Effects of oral contraceptive use on the salivary testosterone and cortisol responses to training sessions and competitions in elite women athletes. Physiology and Behavior, 147, pp.84-90.

Crewther, B.T. et al., 2013. Monitoring salivary testosterone and cortisol concentrations across an international sports competition: data comparison using two enzyme immunoassays and two sample preparations. Clinical biochemistry, 46(4-5), pp.354-8.

Crewther, B.T. et al., 2009. Neuromuscular performance of elite rugby union players and relationships with salivary hormones. Journal of Strength and Conditioning Research, 23(7), pp.2046-2053.

Crewther, B.T. et al., 2012. Relationships between salivary free testosterone and the expression of force and power in elite athletes. Journal of Sports Medicine and Physical Fitness, 52(2), pp.221-227.

Crewther, B.T. et al., 2011. The acute potentiating effects of back squats on athlete performance. Journal of Strength and Conditioning Research, 25(12), pp.3319-3325.

Crewther, B.T. et al., 2011. Two emerging concepts for elite athletes: The short-term effects of testosterone and cortisol on the neuromuscular system and the dose-response training role of these endogenous hormones. Sports Medicine, 41(2), pp.103-123.

Crewther, B.T. et al., 2010. Validating the salivary testosterone and cortisol concentration measures in response to short high-intensity exercise. The Journal of Sports Medicine and Physical Fitness, 50(1), pp.85-92.

Crewther, B.T. \& Cook, C., 2010. Relationships between salivary testosterone and cortisol 
concentrations and training performance in Olympic weightlifters. J Sports Med Phys Fitness, 50(3), pp.371-375.

Crewther, B.T. \& Cook, C.J., 2012. Effects of different post-match recovery interventions on subsequent athlete hormonal state and game performance. Physiology and Behavior, 106(4), pp.471-475.

Crewther, B.T., Kilduff, L.P. \& Cook, C.J., 2014. Trained and untrained males show reliable salivary testosterone responses to a physical stimulus, but not a psychological stimulus. Journal of Endocrinological Investigation, 37(11), pp.1065-1072.

Crisafulli, A. et al., 2011. Ischemic preconditioning of the muscle improves maximal exercise performance but not maximal oxygen uptake in humans. Journal of Applied Physiology, 111(2), pp.530-536.

Cruz, R.S. de O. et al., 2015. Effects of ischemic preconditioning on maximal constant load cycling performance. Journal of Applied Physiology, pp.00498.2015.

Cumming, D.C. et al., 1987. Reproductive hormone responses to resistance exercise. Medicine and Science in Sports and Exercise, 19, pp.234-238.

Cunniffe, B. et al., 2009. An Evaluation of the Physiological Demands of Elite Rugby Union Using Global Positioning System Tracking Software. Journal of Strength and Conditioning Research, 23(4), pp.1195-1203.

Cunniffe, B. et al., 2011. Mucosal immunity and illness incidence in elite rugby union players across a season. Medicine and Science in Sports and Exercise, 43(3), pp.388-397.

Cunniffe, B. et al., 2010. Time course of changes in immuneoendocrine markers following an international rugby game. European Journal of Applied Physiology, 108(1), pp.113-122.

Dabbs, J.M., 1990. Salivary testosterone measurements: reliability across hours, days, and weeks. Physiology \& behavior, 48, pp.83-86.

Dabbs, J.M. \& de La Rue, D., 1991. Salivary testosterone measurements among women: relative magnitude of circadian and menstrual cycles. Hormone research, 35(5), pp.182-184.

Dabbs, J.M. \& La Rue, D. De, 1991. Salivary testosterone measurements among women: Relative magnitude of circadian and menstrual cycles. Hormone Research in Paediatrics, 35(5), pp.182-184.

Damas, F. et al., 2016. Susceptibility to Exercise-Induced Muscle Damage : a Cluster Analysis with a Large Sample. Internaional Journal of Sports Medicine, 37(8), pp.633-640.

Dana, A. et al., 2000. Adenosine A1 receptor induced delayed preconditioning in rabbits. Circulation Research., 86, pp.989-997.

Dankel, S. et al., 2017. The acute muscular response to two distinct blood flow restriction protocols. Physiology International, 104(1), pp.64-76.

Dankel, S.J. et al., 2017. Can blood flow restriction augment muscle activation during high-load training? Clinical Physiology and Functional Imaging, [Epub ahead of print] 
Dascombe, B.J. et al., 2007. The reliability of the i-STAT clinical portable analyser. Journal of Science and Medicine in Sport, 10(3), pp.135-140.

Daskalopoulou, S.S. et al., 2015. The 2015 Canadian Hypertension Education Program Recommendations for Blood Pressure Measurement, Diagnosis, Assessment of Risk, Prevention, and Treatment of Hypertension. Canadian Journal of Cardiology, 31(5), pp.549568.

Davidson, S.M. et al., 2006. Signalling via the reperfusion injury signalling kinase (RISK) pathway links closure of the mitochondrial permeability transition pore to cardioprotection. International Journal of Biochemistry and Cell Biology, 38(3), pp.414-419.

Davies, V., Thompson, K.G. \& Cooper, S.-M., 2009. The Effects of Compression Garments on Recovery. Journal of Strength and Conditioning Research, 23(6), pp.1786-1794.

Davison, R.C. et al., 2000. Assessment of blood lactate: practical evaluation of the Biosen 5030 lactate analyzer. Medicine and Science in Sports and Exercise, 32(1), pp.243-247.

Dawson, E. A et al., 2013. Effects of acute exercise on flow-mediated dilatation in healthy humans. Journal of Applied Physiology, 115(11), pp.1589-98.

Dedovic, K. et al., 2009. The brain and the stress axis: The neural correlates of cortisol regulation in response to stress. NeuroImage, 47(3), pp.864-871.

Delis, K.T. et al., 2000. Optimum intermittent pneumatic compression stimulus for lower-limb venous emptying. European Journal of Vascular and Endovascular Surgery, 19(3), pp.261269.

DeLorey, D.S., Kowalchuk, J.M. \& Paterson, D.H., 2003. Relationship between pulmonary O2 uptake kinetics and muscle deoxygenation during moderate-intensity exercise. Journal of Applied Physiology, 95(1), pp.113-120.

Demarle, A.P. et al., 2001. Decrease of $\mathrm{O}(2)$ deficit is a potential factor in increased time to exhaustion after specific endurance training. Journal of Applied Physiology, 90(3), pp.94753.

Deschodt, J. V., Arsac, L.M. \& Rouard, A.H., 1999. Relative contribution of arms and legs in humans to propulsion in 25-m sprint front-crawl swimming. European Journal of Applied Physiology and Occupational Physiology, 80(3), pp.192-199.

Dideriksen, J.L. \& Farina, D., 2013. Motor unit recruitment by size does not provide functional advantages for motor performance. The Journal of Physiology, 591(24), pp.6139-6156.

Doan, B.K. et al., 2007. Salivary cortisol, testosterone, and T/C ratio responses durinq a 36-hole golf competition. International Journal of Sports Medicine, 28(6), pp.470-479.

Downey, J.M., Davis, A.M. \& Cohen, M. V., 2007. Signaling pathways in ischemic preconditioning. Heart Failure Reviews, 12(3-4), pp.181-188.

Drust, B. et al., 2005. Circadian rhythms in sports performance--an update. Chronobiology International, 22(1), pp.21-44. 
Duncan, C.J., 1987. Role of calcium in triggering rapid ultrastructural damage in muscle: a study with chemically skinned fibres. Journal of Cell Science, 87 ( Pt 4), pp.581-94.

Duncker, D.J. et al., 1993. Role of K+ATP channels in coronary vasodilation during exercise. Circulation, 88(3), pp.1245-53.

Dupont, G. et al., 2010. Effect of 2 Soccer Matches in a Week on Physical Performance and Injury Rate. The American Journal of Sports Medicine, 38(9), pp.1752-1758.

Edwards, D.A. \& Casto, K. V., 2013. Women's intercollegiate athletic competition: Cortisol, testosterone, and the dual-hormone hypothesis as it relates to status among teammates. Hormones and Behavior, 64(1), pp.153-160.

Edwards, D.A. \& Kurlander, L.S., 2010. Hormones and Behavior Women's intercollegiate volleyball and tennis: Effects of warm-up, competition, and practice on saliva levels of cortisol and testosterone. Hormones and Behavior, 58(4), pp.606-613.

Edwards, D.A. \& Neal, J.L.O., 2009. Hormones and Behavior Oral contraceptives decrease saliva testosterone but do not affect the rise in testosterone associated with athletic competition. Hormones and Behavior, 56(2), pp.195-198.

Ehlers, G.G., Ball, T.E. \& Liston, L., 2002. Creatine kinase levels are elevated during 2-a-day practices in collegiate football players. Journal of Athletic Training, 37(2), pp.151-156.

Eisen, A. et al., 2004. Ischemic preconditioning: Nearly two decades of research. A comprehensive review. Atherosclerosis, 172(2), pp.201-210.

Ekstrand, L.G. et al., 2012. Assessing explosive power production using the backwards overhead shot throw and the effects of morning resistance exercise on afternoon performance. Journal of Strength and Conditioning Research, 27(1), pp.1.

Enko, K. et al., 2011. Intermittent arm ischemia induces vasodilatation of the contralateral upper limb. Journal of Physiological Sciences, 61(6), pp.507-513.

Estrada, M. et al., 2003. Testosterone stimulates intracellular calcium release and mitogenactivated protein kinases via a $G$ protein-coupled receptor in skeletal muscle cells. Endocrinology, 144(8), pp.3586-3597.

Evans, C., Vance, S. \& Brown, M., 2010. Short-term resistance training with blood flow restriction enhances microvascular filtration capacity of human calf muscles. Journal of Sports Sciences, 28(9), pp.999-1007.

Fahs, C.A. et al., 2015. Muscular adaptations to fatiguing exercise with and without blood flow restriction. Clinical Physiology and Functional Imaging, 35(3), pp.167-176.

Fahs, C. a et al., 2012. Methodological considerations for blood flow restricted resistance exercise. Journal of Trainology, 1, pp.14-22.

Falvo, M.J. et al., 2009. Repeated bout effect is absent in resistance trained men: An electromyographic analysis. Journal of Electromyography and Kinesiology, 19(6).

Fatela, P. et al., 2016. Acute effects of exercise under different levels of blood-flow restriction on 
muscle activation and fatigue. European Journal of Applied Physiology, 116(5), pp. 985-95.

Favero, T.G., Pessah, I.N. \& Klug, G. a, 1993. Prolonged exercise reduces Ca2+ release in rat skeletal muscle sarcoplasmic reticulum. European Journal of Physiology, 422(5), pp.472475 .

Ferguson, R.A., Dodd, M.J. \& Paley, V.R., 2014. Neuromuscular electrical stimulation via the peroneal nerve is superior to graduated compression socks in reducing perceived muscle soreness following intense intermittent endurance exercise. European Journal of Applied Physiology, pp.2223-2232.

Fernström, M., Tonkonogi, M. \& Sahlin, K., 2004. Effects of acute and chronic endurance exercise on mitochondrial uncoupling in human skeletal muscle. The Journal of Physiology, 554(3), pp.755-763.

Ferreira, T.N. et al., 2016. Ischemic Preconditioning and Repeated Sprint Swimming: A Placebo and Nocebo Study. Medicine and Science in Sports and Exercise, 48(10), pp.1967-1975.

Filaire, E. \& Lac, G., 2000. Dehydroepiandrosterone (DHEA) rather than testosterone shows saliva androgen responses to exercise in elite female handball players. International Journal of Sports Medicine, 21(1), pp.17-20.

Fitschen, P.J. et al., 2014. Perceptual effects and efficacy of intermittent or continuous blood flow restriction resistance training. Clinical Physiology and Functional Imaging, 34, pp.356-363.

Fitzgibbons, P.G. et al., 2012. Safe tourniquet use: a review of the evidence. The Journal of the American Academy of Orthopaedic Surgeons, 20(5), pp.310-9.

Fradkin, A.J., Zazryn, T.R. \& Smoliga, J.M., 2010. Effects of warming-up on physical performance: a systematic review with meta-analysis. Journal of Strength \& Conditioning Research, 24(1), pp.140-148.

Franz, A. et al., 2017. Mechanisms underpinning protection against eccentric exercise-induced muscle damage by ischemic preconditioning. Medical Hypotheses, 98.

Frese, E.M., Fick, A. \& Sadowsky, H.S., 2011. Blood pressure measurement guidelines for physical therapists. Cardiopulmonary Physical Therapy Journal, 22(2), pp.5-12.

Fridén, J. et al., 1983. Adaptive response in human skeletal muscle subjected to prolonged eccentric training. International Journal of Sports Medicine, 4(3), pp.177-83.

Fridén, J., Sjöström, M. \& Ekblom, B., 1983. Myofibrillar Damage Following Intense Eccentric Exercise in Man. International Journal of Sports Medicine, 4(3), pp.170-176.

Fry, A. \& Kraemer, W.J., 1997. Resistance exercise overtraining and overreaching. Neuroendocrine responses. Sports Medicine, 23(2), pp.106-129.

Fry, C.S. et al., 2010. Blood flow restriction exercise stimulates mTORC1 signaling and muscle protein synthesis in older men. Journal of Applied Physiology, 108(5), pp.1199-209.

Fry, R.W. et al., 1991. Monitoring exercise stress by changes in metabolic and hormonal responses over a 24-h period. European Journal of Applied Physiology and Occupational Physiology, 
63(3-4), pp.228-234.

Fryer, R.M. et al., 2000. Ischemic preconditioning in rats: role of mitochondrial K(ATP) channel in preservation of mitochondrial function. American Journal of Physiology Heart and Circulatory Physiology, 278(1), pp.H305-12.

Fryer, R.M., Auchampach, J.. \& Gross, G.J., 2002. Therapeutic receptor targets of ischemic preconditioning. Cardiovascular Research, 55(3), pp.520-525.

Fujimoto, V.Y. et al., 1986. Increased adrenocortical responsiveness to exogenous ACTH in oral contraceptive users. Advances in Contraception: The Official Journal of the Society for the Advancement of Contraception, 2(4), pp.343-353.

Fujita, S. et al., 2007. Blood flow restriction during low-intensity resistance exercise increases S6K1 phosphorylation and muscle protein synthesis. Journal of Applied Physiology, 103(3), pp.903-910.

Fujita, T. et al., 2008. Increased muscle volume and strength following six days of low-intensity resistance training with restricted muscle blood flow. International Journal of KAATSU Training Research, 4, pp.1-8.

Gandevia, S.C., 2001. Spinal and Supraspinal Factors in Human Muscle Fatigue. Physiological Reviews, 81(4), pp.1725-1789.

Gandra, P.G., Nogueira, L. \& Hogan, M.C., 2012. Mitochondrial activation at the onset of contractions in isolated myofibres during successive contractile periods. The Journal of Physiology, 590(15), pp.3597-609.

Garcia, C.A. et al., 2017. Ischemic Preconditioning and Acute Recovery of Performance in Rugby Union Players. Sports Medicine International Open, pp.107-112.

Gastin, P.B., 2001. Energy system interaction and relative contribution during maximal exercise. Sports medicine (Auckland, N.Z.), 31(10), pp.725-741.

Gho, B.C. et al., 1996. Myocardial protection by brief ischemia in noncardiac tissue. Circulation, 94(9), pp.2193-2200.

Ghosh, S., Standen, N.B. \& Galiñanes, M., 2000. Preconditioning the human myocardium by simulated ischemia: Studies on the early and delayed protection. Cardiovascular Research, 45(2), pp.339-350.

Gibson, N. White, J. Neish, M. Murray, A., 2013. Effect of ischemic preconditioning on land based sprinting in team sport athletes. International Journal of Sports Physiology and Performance, 8(6), pp.671-676.

Gissel, H. \& Clausen, T., 2001. Excitation-induced Ca2+ influx and skeletal muscle cell damage. In Acta Physiologica Scandinavica. pp. 327-334.

Gonzalez-Bono, E. et al., 1999. Testosterone, cortisol, and mood in a sports team competition. Hormones and Behavior, 35(1), pp.55-62.

Goto, C. et al., 2003. Effect of different intensities of exercise on endothelium-dependent 
vasodilation in humans: Role of endothelium-dependent nitric oxide and oxidative stress. Circulation, 108(5), pp.530-535.

Goto, K. et al., 2007. Attenuated growth hormone response to resistance exercise with prior sprint exercise. Medicine and Science in Sports and Exercise, 39(1), pp.108-115.

Goto, K. et al., 2005. Prior endurance exercise attenuates growth hormone response to subsequent resistance exercise. European Journal of Applied Physiology, 94, pp.333-338.

Goto, M. et al., 1995. Role of bradykinin in protection of ischemic preconditioning in rabbit hearts. Circulation research, 77(3), pp.611-21.

Gozansky, W.S. et al., 2005. Salivary cortisol determined by enzyme immunoassay is preferable to serum total cortisol for assessment of dynamic hypothalamic-pituitary-adrenal axis activity. Clinical Endocrinology, 63(3), pp.336-341.

Graham, C.A. et al., 2007. Does oral contraceptive-induced reduction in free testosterone adversely affect the sexuality or mood of women? Psychoneuroendocrinology, 32(3), pp.246-255.

Granger, D.A. et al., 1994. Testosterone and conduct problems. Journal of the American Academy of Child \& Adolescent Psychiatry, 33(6), p.908.

Granger, D.A. et al., 2004. The "trouble" with salivary testosterone. Psychoneuroendocrinology, 29(10), pp.1229-1240.

Green, D.J. et al., 2014. Is flow-mediated dilation nitric oxide mediated?: A meta-analysis. Hypertension, 63(2), pp.376-382.

Green, H.J., 1997. Mechanisms of muscle fatigue in intense exercise. Journal of Sports Sciences, 15(3), pp.247-256.

De Groot, P.C.E. et al., 2010. Ischemic preconditioning improves maximal performance in humans. European Journal of Applied Physiology, 108(1), pp.141-146.

Gröschl, M., 2008. Current status of salivary hormone analysis. Clinical Chemistry, 54(11), pp.1759-1769.

Gullich, A. \& Sehmidtbleicher, D., 1996. MVC-induced short-term potentiation of explosive force. New Studies in Athletics, 11(4), pp.67-81.

Gürke, L. et al., 2000. Mechanisms of ischemic preconditioning in skeletal muscle. The Journal of Surgical Research, 94(1), pp.18-27.

Hagar, J.M., Hale, S.L. \& Kloner, R.A., 1991. Effect of preconditioning ischemia on reperfusion arrhythmias after coronary artery occlusion and reperfusion in the rat. Circulation research, 68(1), pp.61-68.

Hajrasouliha, A.R. et al., 2008. Endogenous cannabinoids contribute to remote ischemic preconditioning via cannabinoid CB2 receptors in the rat heart. European Journal of Pharmacology, 579(1-3), pp.246-252.

Häkkinen, K. \& Keskinen, K.L., 1989. Muscle cross-sectional area and voluntary force production characteristics in elite strength- and endurance-trained athletes and sprinters. European 
Journal of Applied Physiology and Occupational Physiology, 59(3), pp.215-220.

Häkkinen, K. \& Pakarinen, a, 1995. Acute hormonal responses to heavy resistance exercise in men and women at different ages. International Journal of Sports Medicine, 16(3), pp.507513.

Hansen, Å.M., Garde, A.H. \& Persson, R., 2008. Sources of biological and methodological variation in salivary cortisol and their impact on measurement among healthy adults: A review. Scandinavian Journal of Clinical and Laboratory Investigation, 68(6), pp.448-458.

Hargens, A.R. et al., 1987. Local compression patterns beneath pneumatic tourniquets applied to arms and thighs of human cadavera. Journal of Orthopaedic Research, 5(2), pp.247-252.

Hargreaves, M. et al., 1998. Muscle metabolites and performance during high-intensity, intermittent exercise. Journal of Applied Physiology, 84(5), pp.1687-1691.

Hastings, M.H. \& Herzog, E.D., 2004. Clock Genes, Oscillators, and Cellular Networks in the Suprachiasmatic Nuclei. Journal of Biological Rhythms, 19(5), pp.400-413.

Hatfield, D.L., Nicoll, J.. \& Kraemer, W.J., 2016. Effects of circadian rhythm on power, force and hormonal response in young men. Journal of Strength \& Conditioning Research, 30(3), pp.725-732.

Hatze, H., 1998. Validity and reliability of methods for testing vertical jumping performance. Journal of Applied Biomechanics, 14(2), pp.127-140.

Hausenloy, D.J. \& Yellon, D.M., 2008. Remote ischaemic preconditioning: Underlying mechanisms and clinical application. Cardiovascular Research, 79(3), pp.377-386.

Heitkamp, H.C., 2015. Training with blood flow restriction. Mechanisms, gain in strength and Safety. Journal of Sports Medicine and Physical Fitness, 55(5).

Hellhammer, D.H., Hubert, W. \& Schürmeyer, T., 1985. Changes in saliva testosterone after psychological stimulation in men. Psychoneuroendocrinology, 10(1), pp.77-81.

Herman, J.P. et al., 2005. Limbic system mechanisms of stress regulation: Hypothalamo-pituitaryadrenocortical axis. Progress in Neuro-Psychopharmacology and Biological Psychiatry, 29(8), pp.1201-1213.

Heusch, G. et al., 2015. Remote ischemic conditioning. Journal of the American College of Cardiology, 65(2), pp.177-195.

Hittinger, E. a et al., 2015. Ischemic preconditioning does not improve peak exercise capacity at sea level or simulated high altitude in trained male cyclists. Applied Physiology, Nutrition, and Metabolism, 7, pp.1-7.

Hoffman, J.R. et al., 2002. Performance, biochemical, and endocrine changes during a competitive football game. Medicine and Science in Sports and Exercise, 34(11), pp.1845-1853.

Hofman, L.F., 2001. Human Saliva as a Diagnostic Specimen. Journal of Nutrition., 131(5), p.1621S-1625.

Hoogeveen, a R. \& Zonderland, M.L., 1996. Relationships between testosterone, cortisol and 
performance in professional cyclists. International Journal of Sports Medicine, 17(6), pp.423-8.

Hopkins, W.G., Hawley, J. A \& Burke, L.M., 1999. Design and analysis of research on sport performance enhancement. Medicine and Science in Sports and Exercise, 31(3), pp.472-485.

Horiuchi, M., 2017. Ischemic preconditioning: Potential impact on exercise performance and underlying mechanisms. Journal of Physical Fitness and Sports Medicine, 6(1), pp.15-23.

Horiuchi, M., Endo, J. \& Thijssen, D.H.J., 2015. Impact of ischemic preconditioning on functional sympatholysis during handgrip exercise in humans. Physiological Reports, 3(2), pp.e12304 e12304.

Horrocks, P.M. et al., 1990. Patterns of ACTH and cortisol pulsatility over twenty-four hours in normal males and females. Clinical Endocrinology, 32(1), pp.127-134.

Hortobagyi, T. \& Denahan, T., 1989. Variability in creatine kinase: Methodological, exercise, and clinically related factors. International Journal of Sports Medicine, 10(2), pp.69-80.

Hoshikawa, M. et al., 2010. Postural instability at a simulated altitude of 5,000 m before and after an expedition to Mt. Cho-Oyu (8,201 m). European Journal of Applied Physiology, 110(3), pp.539-547.

Houmard, J.A., 1991. Impact of Reduced Training on Performance in Endurance Athletes. Sports Medicine, 12(6), pp.380-393.

Howatson, G. \& van Someren, K.A., 2008. The prevention and treatment of exercise-induced muscle damage. Sports medicine (Auckland, N.Z.), 38(6), pp.483-503.

Howatson, G., Van Someren, K. \& Hortobágyi, T., 2007. Repeated bout effect after maximal eccentric exercise. International Journal of Sports Medicine, 28, pp.557-563.

$\mathrm{Hu}$, S. et al., 2004. Differentially expressed protein markers in human submandibular and sublingual secretions. International Journal of Oncology, 25(5), pp.1423-1430.

Hunt, J.E.A., Stodart, C. \& Ferguson, R.A., 2016. The influence of participant characteristics on the relationship between cuff pressure and level of blood flow restriction. European Journal of Applied Physiology, 116(7), pp.1421-1432.

Hunter, S.K., 2016. The relevance of sex differences in performance fatigability. Medicine and Science in Sports and Exercise, 48(11), pp.2247-2256.

Husmann, F. et al., 2017. Impact of blood flow restriction exercise on muscle fatigue development and recovery. Medicine and Science in Sport and Exercise, [Epub ahead of print].

Impellizzeri, F.M. \& Maffiuletti, N. A, 2007. Convergent evidence for construct validity of a 7point likert scale of lower limb muscle soreness. Clinical Journal of Sport Medicine, 17(6), pp.494-496.

Inagaki, Y. et al., 2011. Increase in serum growth hormone induced by electrical stimulation of muscle combined with blood flow restriction. European Journal of Applied Physiology, 111(11), pp.2715-21. 
Incognito, A. V., Burr, J.F. \& Millar, P.J., 2015. The Effects of Ischemic Preconditioning on Human Exercise Performance. Sports Medicine, pp.1-14.

Ingham, S. a. et al., 2013. Improvement of 800-m running performance with prior high-intensity exercise. International Journal of Sports Physiology and Performance, 8(1), pp.77-83.

Jaeschke, H. et al., 1996. Mechanisms of inflammatory liver injury: adhesion molecules and cytotoxicity of neutrophils. Toxicology and Applied Pharmacology, 139(2), pp.213-26.

Jean-St-Michel, E. et al., 2011. Remote Preconditioning Improves Maximal Performance in Highly Trained Athletes. Medicine \& Science in Sports \& Exercise, 43(7), pp.1280-1286.

Jessee, M.B. et al., 2016. Letter to the editor: Applying the blood flow restriction pressure: the elephant in the room. American Journal of Physiology - Heart and Circulatory Physiology, pp.2012-2013.

Jessee, M.B. et al., 2016. The Influence of Cuff Width, Sex, and Race on Arterial Occlusion: Implications for Blood Flow Restriction Research. Sports Medicine, 46(6), pp.913-21.

Jiménez-Reyes, P. \& González-Badillo, J.J., 2011. Monitoring training load through the CMJ in sprints and jump events for optimizing performance in athletics. Cultura, Ciencia y Deporte, 7(18), pp.207-217.

Johnson, S.G., Joplin, G.F. \& Burrin, J.M., 1987. Direct assay for testosterone in saliva: relationship with a direct serum free testosterone assay. Clinica chimica acta; International Journal of Clinical Chemistry, 163(3), pp.309-18.

Johnston, M., Cook, C.J., et al., 2015. Neuromuscular, physiological and endocrine responses to a maximal speed training session in elite games players. European Journal of Sport Science, 15(6), pp.550-556.

Jones, A.M. et al., 2008. Influence of pacing strategy on O2 uptake and exercise tolerance. Scandinavian Journal of Medicine and Science in Sports, 18(5), pp.615-626.

Jones, A.M., Koppo, K. \& Burnley, M., 2003. Effects of Prior Exercise on Metabolic and Gas Exchange Responses to Exercise. Sports Medicine, 33(13), pp.949-971.

Jones, B. et al., 2017. Accessing off-field brains in sport; an applied research model to develop practice. British Journal of Sports Medicine Monthly, pp.3-6.

Jones, D.A. et al., 1986. Experimental human muscle damage: morphological changes in relation to other indices of damage. The Journal of Physiology, 375(1), pp.435-448.

Jones, M.R. et al., 2014. Match play performance characteristics that predict post-match creatine kinase responses in professional rugby union players. BMC Sports Science, Medicine and Rehabilitation, 6(1), p.38.

Joyner, M.J. \& Proctor, D.N., 1999. Muscle blood flow during exercise: the limits of reductionism. Medicine and Science in Sports and Exercise, 31(7), pp.1036-1040.

Kacin, A. et al., 2015. Safety considerations with blood flow restricted resistance training. Annals of Kinesiologiae, 6, pp.3-26. 
Kacin, A. \& Strazar, K., 2011. Frequent low-load ischemic resistance exercise to failure enhances muscle oxygen delivery and endurance capacity. Scandinavian Journal of Medicine \& Science in Sports, 21(6), pp.e231-e241.

Kaeffer, N., Richard, V. \& Thuillez, C., 1997. Delayed coronary endothelial protection 24 hours after preconditioning: role of free radicals. Circulation, 96(7), pp.2311-2316.

Kalogeris, T. et al., 2012. Cell Biology of Ischemia/Reperfusion Injury. International Review of Cellular and Molecular Biology, 98, pp. 229-317.

Kanaley, J.A. et al., 1992. Cortisol levels during prolonged exercise: the influence of menstrual phase and menstrual status. International Journal of Sports Medicine, 13(4), pp.332-336.

Kanaley, J. a et al., 2001. Cortisol and growth hormone responses to exercise at different times of day. The Journal of Clinical Endocrinology and Metabolism, 86(6), pp.2881-2889.

Karabulut, M., Bemben, D.A., et al., 2011. Effects of high-intensity resistance training and lowintensity resistance training with vascular restriction on bone markers in older men. European Journal of Applied Physiology, 111(8), pp.1659-1667.

Karabulut, M. et al., 2010. Neuromuscular fatigue following low-intensity dynamic exercise with externally applied vascular restriction. Journal of Electromyography and Kinesiology, 20(3), pp.440-447.

Karabulut, M., Mccarron, J., et al., 2011. The effects of different initial restrictive pressures used to reduce blood flow and thigh composition on tissue oxygenation of the quadriceps. Journal of Sports Sciences, 29(9), pp.951-958.

Karabulut, M. \& Perez, G., 2013. Neuromuscular response to varying pressures created by tightness of restriction cuff. Journal of Electromyography and Kinesiology, 23(6), pp.14941498.

Katayama, K. et al., 2010. Muscle deoxygenation during sustained and intermittent isometric exercise in hypoxia. Medicine and Science in Sports and Exercise, 42(7), pp.1269-1278.

Kawada, S., 2005. What phenomena do occur in blood flow-restricted muscle? International Journal of KAATSU Training Research, 1(2), pp.37-44.

Kay, D. et al., 2000. Different neuromuscular recruitment patterns during eccentric, concentric and isometric contractions. In Journal of Electromyography and Kinesiology. pp. 425-431.

Kay, D. et al., 2001. Evidence for neuromuscular fatigue during high-intensity cycling in warm, humid conditions. European Journal of Applied Physiology, 84(1-2), pp.115-121.

Keizer, H. A, Poortman, J. \& Bunnik, G.S., 1980. Influence of physical exercise on sex-hormone metabolism. Journal of Applied Physiology, 48(5), pp.765-769.

Keller, D.M. et al., 2004. Inhibition of KATP channel activity augments baroreflex-mediated vasoconstriction in exercising human skeletal muscle. The Journal of Physiology, 561(1), pp.273-282.

Kellmann, M., 2010. Preventing overtraining in athletes in high-intensity sports and 
stress/recovery monitoring. Scandinavian Journal of Medicine and Science in Sports, 20(2), pp.95-102.

Kent-Braun, J.A. et al., 2002. Human skeletal muscle responses vary with age and gender during fatigue due to incremental isometric exercise. Journal of Applied Physiology, 93(5), pp.1813-1823.

Kharbanda, R.K. et al., 2001. Ischemic preconditioning prevents endothelial injury and systemic neutrophil activation during ischemia-reperfusion in humans in vivo. Circulation, 103, pp.1624-1630.

Kharbanda, R.K. et al., 2002. Transient limb ischemia induces remote ischemic preconditioning in vivo. Circulation, 106(23), pp.2881-2883.

Kido, K. et al., 2015. Ischemic preconditioning accelerates muscle deoxygenation dynamics and enhances exercise endurance during the work-to-work test. Physiological Reports, 3, pp.e12395-e12395.

Kilduff, L.P. et al., 2008. Influence of recovery time on post-activation potentiation in professional rugby players. Journal of Sports Science, 26(8), pp.795-802.

Kilduff, L.P. et al., 2007. Postactivation potentiation in professional rugby players: Optimal recovery. Journal of Strength and Conditioning Research, 21(4), 1134-8.

Kilduff, L.P. et al., 2013. Preconditioning strategies to enhance physical performance on the day of competition. International Journal of Sports Physiology and Performance, 8(6), pp.677681.

Kim, E. et al., 2014. Hormone responses to an acute bout of low intensity blood flow restricted resistance exercise in college-aged females. Journal of Sports Science and Medicine, 13(1), pp.91-96.

Kim, H.H., 2007. Regulation of gonadotropin-releasing hormone gene expression. Seminars in Reproductive Medicine, 25(5), pp.313-325.

Kim, S. et al., 2012. Effects of Short Term Low Intensity Resistance Training with Blood Flow Restriction on Bone Markers and Muscle Cross- Sectional Area in Young Men. International Journal of Exercise Science, 5(27), pp.136-147.

Kimura, M. et al., 2007. Repetition of ischemic preconditioning augments endothelium-dependent vasodilation in humans: Role of endothelium-derived nitric oxide and endothelial progenitor cells. Arteriosclerosis, Thrombosis, and Vascular Biology, 27(6), pp.1403-1410.

Kirschbaum, C. et al., 1996. Adrenocortical activation following stressful exercise: Further evidence for attenuated free cortisol responses in women using oral contraceptives. Stress Medicine, 12(3), pp.137-143.

Kirschbaum, C. et al., 1999. Impact of Gender, Menstrual Cycle Phase, and Oral Contraceptives on the Activity of the Hypothalamus-Pituitary-Adrenal Axis. Psychosomatic Medicine, 61(2), pp.154-162. 
Kjeld, T. et al., 2014. Ischemic preconditioning of one forearm enhances static and dynamic apnea. Medicine and Science in Sports and Exercise, 46(1), pp.151-155.

Kluess, H. a \& Wood, R.H., 2005. Heart rate variability and the exercise pressor reflex during dynamic handgrip exercise and postexercise arterial occlusion. The American Journal of the Medical Sciences, 329(3), pp.117-123.

Koch, A. J., Pereira, R. \& Machado, M., 2014. The creatine kinase response to resistance exercise. Journal of musculoskeletal \& neuronal interactions, 14(1), pp.68-77.

Koch, S. et al., 2014. Biomarkers for ischemic preconditioning: finding the responders. Journal of Cerebral Blood Flow and Metabolism: official journal of the International Society of Cerebral Blood Flow and Metabolism, 34(6), pp.933-41.

Kocman, E.A. et al., 2015. Effects of ischemic preconditioning protocols on skeletal muscle ischemia-reperfusion injury. Journal of Surgical Research, 193(2), pp.942-952.

Koh, T.J. \& Escobedo, J., 2004. Cytoskeletal disruption and small heat shock protein translocation immediately after lengthening contractions. American journal of physiology. Cell physiology, 286(3), pp.C713-22.

Kok, J. et al., 2015. Evaluation of point-of-care testing in critically unwell patients: comparison with clinical laboratory analysers and applicability to patients with Ebolavirus infection. Pathology, 47(5), pp.405-9.

Kon, Michihiro. Ikeda, Tatsuaki. Homma, Toshiyuki. Suzuki, Y., 2012. Effects of low-intensity resistance exercise under acute systematic hypoxia on hormonal responses. Journal of Strength \& Conditioning Research, 26(6), pp.1-7.

Konstantinov, I.E. et al., 2005. Remote ischemic preconditioning of the recipient reduces myocardial ischemia-reperfusion injury of the denervated donor heart via a Katp channeldependent mechanism. Transplantation, 79(12), pp.1691-1695.

Konstantinov, I.E. et al., 2004. The remote ischemic preconditioning stimulus modifies inflammatory gene expression in humans. Physiological genomics, 19(1), pp.143-50.

Kooijman, M. et al., 2008. Flow-mediated dilatation in the superficial femoral artery is nitric oxide mediated in humans. The Journal of Physiology, 586(4), pp.1137-1145.

Koopman, R. et al., 2006. Increase in S6K1 phosphorylation in human skeletal muscle following resistance exercise occurs mainly in type II muscle fibers. American Journal of Physiology. Endocrinology and Metabolism, 290(6), pp.E1245-E1252.

Kraemer, R. et al., 2011. Acute effects of remote ischemic preconditioning on cutaneous microcirculation - a controlled prospective cohort study. BMC Surgery, 11(1), p.32.

Kraemer, W.J. et al., 2004. Changes in exercise performance and hormonal concentrations over a big ten soccer season in starters and nonstarters. Journal of Strength \& Conditioning Research, 18(1), pp.121-128.

Kraemer, W.J. et al., 1993. Changes in hormonal concentrations after different heavy-resistance 
exercise protocols in women. Journal of Applied Physiology, 75(2), pp.594-604.

Kraemer, W.J. et al., 1991. Endogenous anabolic hormonal and growth factor responses to heavy resistance exercise in males and females. International journal of sports medicine, 12, pp.228-235.

Kraemer, W.J. et al., 2007. Hormonal responses to a 160-km race across frozen Alaska. British Journal of Sports Medicine, 42(2), pp.116-120.

Kraemer, W.J. et al., 2001. The effect of heavy resistance exercise on the circadian rhythm of salivary testosterone in men. European Journal of Applied Physiology, 84(1-2), pp.13-18.

Kraemer, W.J. \& Ratamess, N.A., 2005. Hormonal responses and adaptations to resistance exercise and training. Sports Med, 35(4), pp.339-361.

Kraus, A.S. et al., 2015. Bilateral Upper Limb Remote Ischemic Preconditioning Improves Anaerobic Power. The Open Sports Medicine Journal, 9, pp.1-6.

Kubo, K. et al., 2006. Effects of low-load resistance training with vascular occlusion on the mechanical properties of muscle and tendon. Journal of Applied Biomechanics, 22(2), pp.112-119.

Kuitunen, S. et al., 2004. Voluntary activation and mechanical performance of human triceps surae muscle after exhaustive stretch-shortening cycle jumping exercise. European Journal of Applied Physiology, 91(5-6), pp.538-544.

Kumar, A.M. et al., 2005. Adrenocortical response to ovine corticotropin-releasing hormone in young men: Cortisol measurement in matched samples of saliva and plasma. Hormone Research, 64(2), pp.55-60.

Kuzuya, T. et al., 1993. Delayed Effects of Sublethal Ischemia on the Acquisition of Tolerance to Ischemia. Circulation Research, 72(6), pp.1293-1299.

Kvorning, T. et al., 2007. Suppression of testosterone does not blunt mRNA expression of myoD, myogenin, IGF, myostatin or androgen receptor post strength training in humans. The Journal of Physiology, 578(2), pp.579-593.

Lalonde, F. \& Curnier, D., 2014. Can Anaerobic Performance Be Improved By Remote Ischemic Preconditioning? Journal of Strength and Conditioning Research, pp.80-85.

Laskey, W.K. \& Beach, D., 2003. Frequency and clinical significance of ischemic preconditioning during percutaneous coronary intervention. Journal of the American College of Cardiology, 42(6), pp.998-1003.

Laurent, C.M. et al., 2011. A practical approach to monitoring recovery: development of a perceived recovery status scale. Journal of Strength \& Conditioning Research, 25(3), pp.620-628.

Laurentino, G. et al., 2008. Effects of strength training and vascular occlusion. International Journal of Sports Medicine, 29(8), pp.664-667.

Laurentino, G.C. et al., 2012. Strength training with blood flow restriction diminishes myostatin 
gene expression. Medicine and Science in Sports and Exercise, 44(3), pp.406-412.

Lauver, J.D. et al., 2017. The effect of eccentric exercise with blood flow restriction on neuromuscular activation, microvascular oxygenation, and the repeated bout effect. European Journal of Applied Physiology, 117(5).

Lawson, C.S. \& Downey, J.M., 1993. Preconditioning: State of the art myocardial protection. Cardiovascular Research, 27(4), pp.542-550.

Leek, B.T. et al., 2001. Effect of acute exercise on citrate synthase activity in untrained and trained human skeletal muscle. American Journal of Physiology. Regulatory, integrative and comparative physiology, 280(2), pp.441-7.

Lefer, a M. \& Lefer, D.J., 1996. The role of nitric oxide and cell adhesion molecules on the microcirculation in ischaemia-reperfusion. Cardiovascular research, 32, pp.743-751.

Lepers, R. et al., 2000. Evidence of neuromuscular fatigue after prolonged cycling exercise. Medicine and Science in Sports and Exercise, 32(11), pp.1880-6.

Libonati, J.R. et al., 1998. Brief periods of occlusion and reperfusion increase skeletal muscle force output in humans. Cardiologia, 43(12), pp.1355-1360.

Liem, D. a et al., 2002. Sites of action of adenosine in interorgan preconditioning of the heart. American journal of physiology. Heart and Circulatory Physiology, 283(1), pp.H29-37.

Liening, S.H. et al., 2010. Salivary testosterone, cortisol, and progesterone: Two-week stability, interhormone correlations, and effects of time of day, menstrual cycle, and oral contraceptive use on steroid hormone levels. Physiology and Behavior, 99(1), pp.8-16.

Lin, H. et al., 2001. Stimulatory effect of lactate on testosterone production by rat Leydig cells. Journal of cellular biochemistry, 83(1), pp.147-54.

Lindsay, A. \& Costello, J., 2016. Realising the Potential of Urine and Saliva as Diagnostic Tools in Sport and Exercise Medicine: A Review. Sports Medicine, 47(1), pp.11-31.

Linnamo, V. et al., 2005. Acute hormonal responses to submaximal and maximal heavy resistance and explosive exercises in men and women. Journal of Strength and Conditioning Research, 19(3), pp.566-571.

Lippi, G. et al., 2009. Measurement of morning saliva cortisol in athletes. Clinical Biochemistry, 42(9), pp.904-906.

Lisbôa, F.D. et al., 2017. The Time Dependence of the Effect of Ischemic Preconditioning on Successive Sprint Swimming Performance. Journal of Science and Medicine in Sport, 20(5), pp.507-511.

Liu, G.S. et al., 1994. Evidence that the adenosine A3 receptor may mediate the protection afforded by preconditioning in the isolated rabbit heart. Cardiovascular Research, 28(7), pp.10571061.

Liu, G.S. et al., 1991. Protection against infarction afforded by preconditioning is mediated by A1 adenosine receptors in rabbit heart. Circulation, 84(1), pp.350-6. 
Lochner, A. et al., 2002. Nitric oxide triggers classic ischemic preconditioning. Annals of the New York Academy of Sciences, 962, pp.402-414.

Loenneke, J.P. et al., 2011. Acute and chronic testosterone response to blood flow restricted exercise. Hormone and Metabolic Research, 43(10), pp.669-73.

Loenneke, J.P. et al., 2013. Blood flow restricrion pressure recommendations: a tale of two cuffs. Clinical Physiology and Functional Imaging, 33(4), pp.325-327.

Loenneke, J.P. et al., 2014. Blood flow restriction: effects of cuff type on fatigue and perceptual responses to resistance exercise. Acta Physiologica Hungarica, 101(2), pp.158-66.

Loenneke, J.P., et al., 2011. Blood flow restriction: The metabolite/volume threshold theory. Medical Hypotheses, 77(5), pp.748-752.

Loenneke, J.P. et al., 2013. Blood flow restriction does not result in prolonged decrements in torque. European Journal of Applied Physiology, 113(4), pp.923-931.

Loenneke, J.P. et al., 2015. Blood flow restriction in the upper and lower limbs is predicted by limb circumference and systolic blood pressure. European Journal of Applied Physiology, 115(2), pp.397-405.

Loenneke, J.P. et al., 2012. Blood flow restriction reduces time to muscular failure. European Journal of Sport Science, 12(3), pp.238-243.

Loenneke, J.P. et al., 2012. Effects of cuff width on arterial occlusion: implications for blood flow restricted exercise. European Journal of Applied Physiology, 112(8), pp.2903-2912.

Loenneke, J.P. et al., 2015. Effects of exercise with and without different degrees of blood flow restriction on torque and muscle activation. Muscle \& nerve, 51(5), pp.713-721.

Loenneke, J.P. et al., 2012. Low intensity blood flow restriction training: a meta-analysis. European Journal of Applied Physiology, 112(5), pp.1849-1859.

Loenneke, J.P. et al., 2011. Potential safety issues with blood flow restriction training. Scandinavian Journal of Medicine \& Science in Sports, 21(4), pp.510-518.

Loenneke, J.P. et al., 2015. The effects of resistance exercise with and without different degrees of blood-flow restriction on perceptual responses. Journal of Sports Sciences, 33(14), pp.1472-1479.

Loenneke, J.P. et al., 2012. Time under tension decreased with blood flow-restricted exercise. Clinical Physiology and Functional Imaging, 32(4), pp.268-73.

Loenneke, J.P. \& Abe, T., 2012. Does blood flow restricted exercise result in prolonged torque decrements and muscle damage? European Journal of Applied Physiology, 112(9), pp.34453446.

Loenneke, J.P. \& Pujol, T.J., 2009. The Use of Occlusion Training to Produce Muscle Hypertrophy. Strength and Conditioning Journal, 31(3), pp.77-84.

Loenneke, J.P., Thiebaud, R.S. \& Abe, T., 2014. Does blood flow restriction result in skeletal muscle damage? A critical review of available evidence. Scandinavian Journal of Medicine 
\& Science in Sports, pp.1-8.

Lott, F.D., Guo, P. \& Toombs, C.F., 1996. Reduction in infarct size by ischemic preconditioning persists in a chronic rat model of myocardial ischemia-reperfusion injury. Pharmacology, 52(2), pp.113-118.

Loukogeorgakis, S.P. et al., 2005. Remote Ischemic Preconditioning Provides Early and Late Protection Against Endothelial Ischemia-Reperfusion Injury in Humans Role of the Autonomic Nervous System. Journal of American College of Cardiology, 46(3), pp.16-18.

Lovering, R.M. \& De Deyne, P.G., 2004. Contractile function, sarcolemma integrity, and the loss of dystrophin after skeletal muscle eccentric contraction-induced injury. American Journal of Physiology. Cell physiology, 286, pp.C230-C238.

Lowery, R.P. et al., 2014. Practical blood flow restriction training increases muscle hypertrophy during a periodized resistance training programme. Clinical Physiology and Functional Imaging, 34(4), pp.317-321.

Lu, S.S., et al. 1997. Lactate and the effects of exercise on testosterone secretion: evidence for the involvement of a cAMP-mediated mechanism. Medicine \& Science in Sports \& Exercise, 28(8), pp.1048-1054.

Luebbers, P.E. et al., 2014. The effects of a 7-week practical blood flow restriction program on well-trained collegiate athletes. Journal of Strength \& Conditioning Research, 28(8), pp.2270-2280.

Lupien, S.J. et al., 2007. The effects of stress and stress hormones on human cognition: implications for the field of brain and cognition. Brain and Cognition, 65(3), pp.209-237.

Macintosh, B.R. \& Rassier, D.E., 2002. What Is Fatigue? Canadian Journal of Applied Physiology, 27(1), pp.42-55.

Maczewski, M. et al., 2004. Endothelial protection from reperfusion injury by ischemic preconditioning and diazoxide involves a SOD-like anti-O2 - mechanism. Journal of Physiology and Pharmacology, 55(3), pp.537-550.

Madarame, H. et al., 2008. Cross-transfer effects of resistance training with blood flow restriction. Medicine and Science in Sports and Exercise, 40(2), pp.258-263.

Madarame, H., Sasaki, K. \& Ishii, N., 2010. Endocrine responses to upper- and lower-limb resistance exercises with blood flow restriction. Acta physiologica Hungarica, 97(2), pp.192-200.

Marocolo, M. et al., 2015. Are the Beneficial Effects of Ischemic Preconditioning on Performance Partly a Placebo Effect? International Journal of Sports Medicine, 36(10), pp.822-825.

Marocolo, M. et al., 2015. Ischemic Preconditioning and Placebo Intervention Improves Resistance Exercise Performance. Journal of Strength and Conditioning Research, pp.14621469.

Marocolo, M. et al., 2016. Myths and Facts About the Effects of Ischemic Preconditioning on 
Performance. International Journal of Sports Medicine, 37, pp.87-96.

Maroulis, G.B. \& Triantafillidis, I.K., 2006. Polycystic ovarian disease: The adrenal connection. In Pediatric Endocrinology Reviews. pp. 205-207.

Marx, J.O. et al., 2001. Low-volume circuit versus high-volume periodized resistance training in women. Medicine and Science in Sports and Exercise, pp.635-643.

Maso, F., 2004. Salivary testosterone and cortisol in rugby players: correlation with psychological overtraining items. British Journal of Sports Medicine, 38(3), pp.260-263.

Mattocks, K.T. et al., 2017. The effects of upper body exercise across different levels of blood flow restriction on arterial occlusion pressure and perceptual responses. Physiology \& Behavior, 171, pp.181-186.

McCall, G.E. et al., 1999. Acute and chronic hormonal responses to resistance training designed to promote muscle hypertrophy. Canadian Journal of Applied Physiology, 24(1), pp.96-107.

McCrary, J.M., Ackermann, B.J. \& Halaki, M., 2015. A systematic review of the effects of upper body warm-up on performance and injury. British Journal of Sports Medicine, pp.1-9.

McCully, J. et al., 2006. Age- and gender related differences in ischemia/reperfusion injury and cardioprotection: effects of diazoxide. Annals of Thoracic Surgery, 82(1), pp.117-123.

McCully, K.K. \& Faulkner, J. A, 1985. Injury to skeletal muscle fibers of mice following lengthening contractions. Journal of Applied Physiology, 59(1), pp.119-126.

McEwen, B.S., 2000. The neurobiology of stress: From serendipity to clinical relevance. Brain Research, 886(1-2), pp.172-189.

McGowan, C.J., Pyne, D.B., et al., 2016. Elite sprint swimming performance is enhanced by completion of additional warm-up activities. Journal of Sports Sciences, 24(2), pp.1-7.

McGowan, C.J., Thompson, K.G., et al., 2016. Heated jackets and dryland-based activation exercises used as additional warm-ups during transition enhance sprint swimming performance. Journal of Science and Medicine in Sport, 19(4), pp.354-358.

McGowan, C.J. et al., 2016. Morning exercise enhances afternoon sprint swimming performance. International Journal of Sports Physiology and Performance.

McLellan, C.P., Lovell, D.I. \& Gass, G.C., 2010. Creatine kinase and endocrine responses of elite players pre, during, and post rugby league match play. Journal of Strength and Conditioning Research, 24(11), pp.2908-2919.

McNeil, P.L. \& Khakee, R., 1992. Disruptions of muscle fiber plasma membranes. Role in exercise-induced damage. The American Journal of Pathology, 140(5), pp.1097-1109.

Meneghel, A.J. et al., 2013. Review of the Repeated Bout Effect in Trained and Untrained men Review of the Repeated Bout Effect in Trained and Untrained men. International Journal of Sports Science, 3(5), pp.147-156.

Meyer, R.A., 2006. Does blood flow restriction enhance hypertrophic signaling in skeletal muscle? Journal of Applied Physiology, 100(5), pp.1443-1444. 
Miller, W.L., 1988. Molecular biology of steroid hormone synthesis. Endocrine reviews, 9(3), pp.295-318.

Millet, G. et al., 2000. Influence of ultra-long-term fatigue on the oxygen cost of two types of locomotion. European Journal of Applied Physiology, 83(4-5), pp.376-380.

Millet, G.Y. et al., 2003. Alteration of neuromuscular function after a prolonged road cycling race. International Journal of Sports Medicine, 24(3), pp.190-194.

Millet, G.Y. et al., 2002. Alterations of neuromuscular function after an ultramarathon. Journal of Applied Physiology, 92(2), pp.486-492.

Mittal, P., Shenoy, S. \& Sandhu, J.S., 2008. Effect of different cuff widths on the motor nerve conduction of the median nerve: an experimental study. Journal of Orthopaedic Surgery and Research, 3(1), p.1.

Montgomery, P.G. et al., 2008. The effect of recovery strategies on physical performance and cumulative fatigue in competitive basketball. Journal of Sports Sciences, 26(11), pp.11351145.

Moore, D. et al., 2004. Neuromuscular adaptations in human muscle following low intensity resistance training with vascular occlusion. European Journal of Applied Physiology, 92(45), pp.399-406.

Moreira, A. et al., 2009. Salivary cortisol in top-level professional soccer players. European Journal of Applied Physiology, 106(1), pp.25-30.

Moritani, T. et al., 1992. Oxygen availability and motor unit activity in humans. European Journal of Applied Physiology and Occupational Physiology, 64(6), pp.552-556.

Morley, J.E. et al., 2006. Validation of salivary testosterone as a screening test for male hypogonadism. Aging Male, 9(3), pp.165-169.

Morouço, P. et al., 2011. Relationship between tethered forces and the four swimming techniques performance. Journal of Applied Biomechanics, 27(2), pp.161-169.

Mouser, J.G. et al., 2017. Brachial blood flow under relative levels of blood flow restriction is decreased in a nonlinear fashion. Clinical Physiology and Functional Imaging. [Epub ahead of print]

Murphy, M.E. \& Brayden, J.E., 1995. Nitric oxide hyperpolarizes rabbit mesenteric arteries via ATP-sensitive potassium channels. The Journal of Physiology, 486 (1), pp.47-58.

Murphy, M.N. et al., 2011. Cardiovascular regulation by skeletal muscle reflexes in health and disease. American Journal of Physiology, Heart and Circulation, 301(4), pp.H1191-204.

Murphy, T. et al., 2010. Transcriptional responses in the adaptation to ischaemia-reperfusion injury: a study of the effect of ischaemic preconditioning in total knee arthroplasty patients. Journal of Translational Medicine, 8(1), p.46.

Murry, C.E. et al., 1990. Ischemic preconditioning slows energy metabolism and delays ultrastructural damage during a sustained ischemic episode. Circulation research, 66(4), 
pp.913-931.

Murry, C.E., Jennings, R.B. \& Reimer, K.A., 1986. Preconditioning with ischemia: a delay of lethal cell injury in ischemic myocardium. Circulation, 74(5), pp.1124-1136.

Nakajima, T. et al., 2006. Use and safety of KAATSU training:Results of a national survey. International Journal of KAATSU Training Research, 2(1), pp.5-13.

Nandagopal, K., Dawson, T.M. \& Dawson, V.L., 2001. Critical role for nitric oxide signaling in cardiac and neuronal ischemic preconditioning and tolerance. The Journal of Pharmacology and Experimental Therapeutics, 297(2), pp.474-478.

Nédélec, M. et al., 2012. Recovery in soccer: Part 1. Sports Medicine, 43(1), pp.9-22.

Nédélec, M. et al., 2013. Recovery in soccer: Part II-recovery strategies. Sports Medicine, 43(1), pp.9-22.

Neiva, H.P. et al., 2014. Warm-up and performance in competitive swimming. Sports Medicine, 44(3), pp.319-330.

Neto, G.R. et al., 2014. Effects of High-Intensity Blood Flow Restriction Exercise on Muscle Fatigue. Journal of Human Kinetics, 41(1), pp.163-172.

Newton, M.J. et al., 2008. Comparison of Responses to Strenuous Eccentric Exercise of the Elbow Flexors Between Resistance-Trained and Untrained Men. Journal of Strength and Conditioning Research, 22(2), pp.597-607.

Nindl, B.C. et al., 2001. Testosterone responses after resistance exercise in women: influence of regional fat distribution. International Journal of Sport Nutrition and Exercise Metabolism, 11(4), pp.451-65.

Noakes, T.D., 2011. Is it time to retire the A.V. hill model?: A rebuttal to the article by professor Roy Shephard. Sports Medicine, 41(4), pp.263-277.

Noakes, T.D., 2000. Physiological models to understand exercise fatigue and the adaptations that predict or enhance athletic performance. Scandinavian Journal of Medicine and Science in Sports, 10(3), pp.123-145.

Northey, J M. et al., 2016. Vascular Occlusion and Sequential Compression for Recovery Post Resistance Exercise. Journal of Strength \& Conditioning Research, 30(2), pp.533-539.

O'connor, P.J. \& Corrigan, D.L., 1987. Influence of short-term cycling on salivary cortisol levels. Medicine and Science in Sports and Exercise, 19(3), pp.224-228.

Okuno, N.M. et al., 2014. Cardiac autonomic recovery after a single session of resistance exercise with and without vascular occlusion. Journal of Strength and Conditioning Research, 28(4), pp.1143-1150.

Ovize, M., Thibault, H. \& Przyklenk, K., 2013. Myocardial conditioning: Opportunities for clinical translation. Circulation Research, 113(4), pp.439-450.

Owen, N.J, et al., 2014. Development of a Criterion Method to Determine Peak Mechanical Power Output in a Countermovement Jump. Journal of Strenth and Conditioning Research, 28(6), 
pp.1552-1558.

Oxman, T. et al., 1997. Limb ischemia preconditions the heart against reperfusion tachyarrhythmia. The American Journal of Physiology, 273(4 Pt 2), pp.H1707-H1712.

Padilla, J. et al., 2011. Vascular effects of exercise: endothelial adaptations beyond active muscle beds. Physiology, 26(3), pp.132-45.

Page, W., Swan, R. \& Patterson, S.D., 2017. The effect of intermittent lower limb occlusion on recovery following exercise-induced muscle damage: A randomized controlled trial. Journal of Science and Medicine in Sport.

Paixão, R.C., Ribeiro da Mota, G. \& Marocolo, M., 2014. Acute Effect of Ischemic Preconditioning is Detrimental to Anaerobic Performance in Cyclists. International Journal of Sports Medicine, pp.912-915.

Pang, C.Y. et al., 1995. Acute ischaemic preconditoning protects against skeletal muscle infarction in the pig. Cardivascular Research, 29, pp.782-788.

Pang, C.Y. et al., 1997. Effector mechanism of adenosine in acute ischemic preconditioning of skeletal muscle against infarction. The American Journal of Physiology, 273(3 Pt 2), pp.R887-95.

Papacosta, E. \& Nassis, G.P., 2011. Saliva as a tool for monitoring steroid, peptide and immune markers in sport and exercise science. Journal of Science and Medicine in Sport, 14(5), pp.424-434.

Paradis-Deschenes, P., Joanisse, D.R. \& Billaut, F., 2016. Ischemic preconditioning increases muscle perfusion, oxygen uptake and force in strength trained athletes. Applied Physiology, Nutrition, and Metabolism, 4(9), pp. 938-44.

Paradis-Deschênes, P., Joanisse, D.R. \& Billaut, F., 2017. Sex-specific impact of ischemic preconditioning on tissue oxygenation and maximal concentric force. Frontiers in Physiology, 7.

Parker, B.A. et al., 2007. Sex differences in leg vasodilation during graded knee extensor exercise in young adults. Journal of Applied Physiology, 103(5), pp.1583-91.

Patel, H.H. et al., 2002. Cardioprotection at a distance: Mesenteric artery occlusion protects the myocardium via an opioid sensitive mechanism. Journal of Molecular and Cellular Cardiology, 34(10), pp.1317-1323.

Patterson, S.D. et al., 2015. The effect of ischemic preconditioning on repeated sprint cycling performance. Medicine and Science in Sports and Exercise, 47(8), pp.1652-1658.

Patterson, S.D. \& Brandner, C.R., 2017. The role of blood flow restriction training for applied practitioners: A questionnaire-based survey. Journal of Sports Sciences, 36(2), pp.123-130.

Peake, J.M. et al., 2005. Exercise-induced muscle damage, plasma cytokines, and markers of neutrophil activation. Medicine and Science in Sports and Exercise, 37(5), pp.737-745.

Pearce, A.J., Rowe, G.S. \& Whyte, D.G., 2012. Neural conduction and excitability following a 
simple warm up. Journal of Science and Medicine in Sport, 15(2), pp.164-168.

Pearson, S.J. \& Hussain, S.R., 2015. A Review on the Mechanisms of Blood-Flow Restriction Resistance Training-Induced Muscle Hypertrophy. Sports Medicine, 45(2), pp.187-200.

Pell, T.J. et al., 1998. Renal ischemia preconditions myocardium: role of adenosine receptors and ATP-sensitive potassium channels. The American journal of physiology, 275(5 Pt 2), pp.H1542-H1547.

Peralta, C. et al., 2001. Preconditioning protects against systemic disorders associated with hepatic ischemia-reperfusion through blockade of tumor necrosis factor-induced P-selectin upregulation in the rat. Hepatology, 33(1), pp.100-113.

Perez-Pinzon, M.A., 2004. Neuroprotective effects of ischemic preconditioning in brain mitochondria following cerebral ischemia. Journal of Bioenergetics and Biomembranes. pp. $323-327$.

Pickering, T.G. et al., 2005. Recommendations for Blood Pressure Measurement in Humans: An AHA Scientific Statement from the Council on High Blood Pressure Research Professional and Public Education Subcommittee. The Journal of Clinical Hypertension, 7(2), pp.102109.

Pinar, S. et al., 2012. Different recovery methods and muscle performance after exhausting exercise: Comparison of the effects of electrical muscle stimulation and massage. Biology of Sport, 29(4), pp.269-275.

Pinniger, G.J., Steele, J.R. \& Groeller, H., 2000. Does fatigue induced by repeated dynamic efforts affect hamstring muscle function? Medicine and Science in Sports and Exercise, 32(3), pp.647-653.

Pope, Z., Willardson, J. \& Schoenfeld, B., 2013. Exercise and blood flow restriction. Journal of Strength and Conditioning Research, 27(10), pp. 2914-26.

Pritchard, B.T. et al., 2017. Factors affecting Measurement of salivary cortisol and secretory immunoglobulin a in Field studies of athletes. Frontiers in Endocrinology, 8, pp.1-7.

Proctor, G.B. \& Carpenter, G.H., 2007. Regulation of salivary gland function by autonomic nerves. Autonomic Neuroscience: Basic and Clinical, 133(1), pp.3-18.

Przyklenk, K. et al., 1993. Regional ischemic "preconditioning" protects remote virgin myocardium from subsequent sustained coronary occlusion. Circulation, 87(3), pp.893-9.

Pyne, D.B., Trewin, C.B. \& Hopkins, W.G., 2004. Progression and variability of competitive performance of Olympic swimmers. Journal of Sports Sciences, 22(7), pp.613-620.

Randhawa, P.K., Bali, A. \& Jaggi, A.S., 2015. RIPC for multiorgan salvage in clinical settings: Evolution of concept, evidences and mechanisms. European Journal of Pharmacology, 746, pp.317-332.

Rantonen, P.J. et al., 2000. Growth hormone and cortisol in serum and saliva. Acta odontologica Scandinavica, 58(6), pp.299-303. 
Rassaf, T. et al., 2014. Circulating nitrite contributes to cardioprotection by remote ischemic preconditioning. Circulation Research, 114(10), pp.1601-1610.

Ratamess, N.A. et al., 2005. Androgen receptor content following heavy resistance exercise in men. Journal of Steroid Biochemistry and Molecular Biology, 93(1), pp.35-42.

Reeves, G. V et al., 2006. Comparison of hormone responses following light resistance exercise with partial vascular occlusion and moderately difficult resistance exercise without occlusion. Journal of Applied Physiology, 101(6), pp.1616-1622.

Rehak, N.N., Cecco, S.A. \& Csako, G., 2000. Biochemical composition and electrolyte balance of "unstimulated" whole human saliva. Clinical Chemistry and Laboratory Medicine, 38(4), pp.335-343.

Reilly, T. \& Waterhouse, J., 2009. Sports performance: Is there evidence that the body clock plays a role? European Journal of Applied Physiology, 106(3), pp.321-332.

Remensnyder, J.P., Mitchell, J.H. \& Sarnoff, S.J., 1962. Functional sympatholysis during muscular activity. Observations on influence of carotid sinus on oxygen uptake. Circulation research, 11, pp.370-380.

Renzi, C P. Tanaka, H. Sugawara, J., 2011. Effects of leg blood flow restriction during walking on cardiovascular function. Medicine \& Science in Sports \& Exercise, 42(4), pp.726-732.

Riksen, N.P., Smits, P. \& Rongen, G.A., 2004. Ischaemic preconditioning: From molecular characterisation to clinical application - Part I. Netherlands Journal of Medicine, 62(10), pp.353-363.

Rockey, D.C. \& Chung, J.J., 1995. Inducible nitric oxide synthase in rat hepatic lipocytes and the effect of nitric oxide on lipocyte contractility. Journal of Clinical Investigation, 95(3), pp.1199-1206.

Rodriguez, F. \& Mader, A., 2003. Energy metabolism during 400 and 100-m crawl swimming: computer simulation based on free swimming measurement. Biomechanics and Medecine in Swimming VIII, (January 2003), pp.373-390.

Roelfsema, F. et al., 1993. Sex-dependent alteration in cortisol response to endogenous adrenocorticotropin. Journal of Clinical Endocrinology and Metabolism, 77(1), pp.234-240.

Rossow, L.M. et al., 2012. Cardiovascular and perceptual responses to blood-flow-restricted resistance exercise with differing restrictive cuffs. Clinical Physiology and Functional Imaging, 32(5), pp.331-337.

Russ, D.W. \& Kent-Braun, J. a, 2003. Sex differences in human skeletal muscle fatigue are eliminated under ischemic conditions. Journal of Applied Physiology, 94, pp.2414-2422.

Russell, M R. et al., 2015. A comparison of different modes of morning priming exercise on afternoon performance. International Journal of Sports Physiology and Performance, 11(6), pp.763-7.

Russell, M. et al., 2016. Relationships between match activities and peak power output and 
Creatine Kinase responses to professional reserve team soccer match-play. Human Movement Science, 45, pp.96-101.

Russell, M. et al., 2015. Responses to a 120 min reserve team soccer match: a case study focusing on the demands of extra time. Journal of Sports Sciences, 33(20), pp.2133-2139.

Sabino-Carvalho, J.L.C. et al., 2016. Effect of Ischemic Preconditioning on Endurance Performance Does Not Surpass Placebo. Medicine and Science in Sports and Exercise, (25), pp.12-14.

Sack, S. et al., 1993. Ischaemic preconditioning - time course of renewal in the pig. Cardiovascular Research, 27(4), pp.551-555.

Saito, T. et al., 2004. Ischemic preconditioning improves oxygenation of exercising muscle in vivo. Journal of Surgical Research, 120(1), pp.111-118.

Sakamaki, M. et al., 2008. Blood pressure response to slow walking combined with KAATSU in the elderly. International Journal of KAATSU Training Research, 4(1), pp.17-20.

Sale, M. V., Ridding, M.C. \& Nordstrom, M.A., 2008. Cortisol Inhibits Neuroplasticity Induction in Human Motor Cortex. Journal of Neuroscience, 28(33), pp.8285-8293.

Salvador, A F. et al., 2016. Ischemic preconditioning and exercise performance: A systematic review and meta-analysis. International Journal of Sport Nutrition and Exercise Metabolism, 11(1), pp.4-14.

Salvador, A. et al., 2003. Anticipatory cortisol, testosterone and psychological responses to judo competition in young men. Psychoneuroendocrinology, 28(3), pp.364-375.

Schipper, R.G., Silletti, E. \& Vingerhoeds, M.H., 2007. Saliva as research material: Biochemical, physicochemical and practical aspects. Archives of Oral Biology, 52(12), pp.1114-1135.

Schoenfeld, B.J., 2013. Potential mechanisms for a role of metabolic stress in hypertrophic adaptations to resistance training. Sports Medicine, 43(3), pp.179-194.

Schoenfeld, B.J., 2010. The mechanisms of muscle hypertrophy and their application to resistance training. Journal of Strength \& Conditioning Research, 24(10), pp.2857-2872.

Schramm, W. et al., 1993. Testosterone concentration is increased in whole saliva, but not in ultrafiltrate, after toothbrushing. Journal of Clinical chemistry, 39, pp.519-521.

Schultheiss, O.C., Dargel, A. \& Rohde, W., 2003. Implicit motives and gonadal steroid hormones: Effects of menstrual cycle phase, oral contraceptive use, and relationship status. Hormones and Behavior, 43(2), pp.293-301.

Schwartz, E.B. \& Granger, D.A., 2004. Transferrin Enzyme Immunoassay for Quantitative Monitoring of Blood Contamination in Saliva. Clinical Chemistry, 50(3), pp.654-656.

Scott, B.R. et al., 2015. Blood flow restricted exercise for athletes: A review of available evidence. Journal of Science and Medicine in Sport / Sports Medicine Australia, 19(5), pp.360-7.

Scott, B.R. et al., 2015. Exercise with Blood Flow Restriction: An Updated Evidence-Based Approach for Enhanced Muscular Development. Sports Medicine, 45(3), pp.313-325. 
Scott, B.R. et al., 2014. Hypoxia and Resistance Exercise: A Comparison of Localized and Systemic Methods. Sports Medicine, 44(8), pp.1037-1054.

Seeger, J.P.H. et al., 2017. Is Delayed Ischemic Preconditioning As Effective on Running Performance During a 5-Km Time Trial As Acute IPC? Journal of Science and Medicine in Sport, 20(2), pp.208-212.

Seitz, L.B. et al. 2015. Application of Methods of Inducing Postactivation Potentiation During the Preparation of Rugby Players. National Strength and Conditioning Association, 37(1), pp.4049.

Sellers, J.G., Mehl, M.R. \& Josephs, R.A., 2007. Hormones and personality: Testosterone as a marker of individual differences. Journal of Research in Personality, 41(1), pp.126-138.

Sharma, V. et al., 2014. Characterization of acute ischemia-related physiological responses associated with remote ischemic preconditioning: a randomized controlled, crossover human study. Physiological Reports, 2(11), pp.1-11.

Sharma, V. et al., 2015. From Protecting the Heart to Improving Athletic Performance - the Benefits of Local and Remote Ischaemic Preconditioning. Cardiovascular Drugs and Therapy, 29(6), pp.573-588.

Sharma, V., Bell, R.M. \& Yellon, D.M., 2012. Targeting reperfusion injury in acute myocardial infarction: a review of reperfusion injury pharmacotherapy. Expert Opinion on Pharmacotherapy, 13(8), pp.1153-75.

Sharp, R.L., Williams, D.J. \& Bevan, L., 1991. Effects of controlled frequency breathing during exercise on blood gases and acid-base balance. International Journal of Sports Medicine, 12(1), pp.62-5.

Shearer, D.A. et al., 2015. Measuring Recovery in Elite Rugby Players: The Brief Assessment of Mood, Endocrine Changes, and Power. Research Quarterly for Exercise and Sport, 86(4), pp.379-86.

Shinohara, M. et al., 1998. Efficacy of tourniquet ischemia for strength training with low resistance. European Journal of Applied Physiology Occupational Physiology, 77, pp.189191.

Shirtcliff, E. A, Granger, D. a \& Likos, A., 2002. Gender differences in the validity of testosterone measured in saliva by immunoassay. Hormones and Behavior, 42(1), pp.62-69.

Singh, D. \& Chopra, K., 2004. Evidence of the role of angiotensin AT(1) receptors in remote renal preconditioning of myocardium. Methods and Findings in Experimental and Clinical Pharmacology, 26(2), pp.117-22.

Sinha-Hikim, I. et al., 2004. Androgen Receptor in Human Skeletal Muscle and Cultured Muscle Satellite Cells: Up-Regulation by Androgen Treatment. Journal of Clinical Endocrinology and Metabolism, 89(10), pp.5245-5255.

Skurvydas, A. et al., 2007. Bimodal recovery of quadriceps muscle force within 24 hours after 
sprint cycling for 30 seconds. Medicina (Kaunas, Lithuania), 43(3), pp.226-34.

Slysz, J., Stultz, J. \& Burr, J.F., 2016. The efficacy of blood flow restricted exercise: A systematic review \&amp; meta-analysis. Journal of Science and Medicine in Sport, 19(8), pp.669-675.

Sporer, B.C., Cote, A. \& Sleivert, G., 2012. Warm-up practices in elite snowboard athletes. International Journal of Sports Physiology and Performance, 7(3), pp.295-297.

St Clair Gibson, A., Lambert, M.I. \& Noakes, T.D., 2001. Neural control of force output during maximal and submaximal exercise. Sports Medicine, 31(9), pp.637-650.

Steele, D.S. \& Duke, A.M., 2003. Metabolic factors contributing to altered Ca2+ regulation in skeletal muscle fatigue. Acta Physiol Scand., 179(1), pp.39-48.

Stupnicki, R. \& Obminski, Z., 1992. Glucocorticoid response to exercise as measured by serum and salivary cortisol. European Journal of Applied Physiology and Occupational Physiology, 65(6), pp.546-549.

Suga, T. et al., 2010. Dose effect on intramuscular metabolic stress during low-intensity resistance exercise with blood flow restriction. Journal of Applied Physiology, 108(6), pp.1563-1567.

Suga, T. et al., 2012. Effect of multiple set on intramuscular metabolic stress during low-intensity resistance exercise with blood flow restriction. European Journal of Applied Physiology, 112(11), pp.3915-3920.

Suga, T. et al., 2009. Intramuscular metabolism during low-intensity resistance exercise with blood flow restriction. Journal of Applied Physiology, 106(4), pp.1119-1124.

Sumide, T. et al., 2009. Effect of resistance exercise training combined with relatively low vascular occlusion. Journal of Science and Medicine in Sport, 12(1), pp.107-112.

Sun, J.Z. et al., 1996. Evidence for an essential role of reactive oxygen species in the genesis of late preconditioning against myocardial stunning in conscious pigs. Journal of Clinical Investigation, 97(2), pp.562-576.

Suzuki, M., 2004. Effect of incorporating low intensity exercise into the recovery period after a rugby match. British Journal of Sports Medicine, 38(4), pp.436-440.

Suzuki, Y. et al., 2002. High level of skeletal muscle carnosine contributes to the latter half of exercise performance during 30-s maximal cycle ergometer sprinting. The Japanese Journal of Physiology, 52(2), pp.199-205.

Tafet, G.E. et al., 2001. Correlation between cortisol level and serotonin uptake in patients with chronic stress and depression. Cognitive, Affective \& Behavioral Neuroscience, 1(4), pp.388393.

Takada, S. et al., 2012. Blood Flow Restriction Exercise in Sprinters and Endurance Runners. Medicine \& Science in Sports \& Exercise, 44(3), pp.413-419.

Takano, H. et al., 2005. Hemodynamic and hormonal responses to a short-term low-intensity resistance exercise with the reduction of muscle blood flow. European Journal of Applied Physiology, 95(1), pp.65-73. 
Takarada, Y., Takazawa, H., et al., 2000. Effects of resistance exercise combined with moderate vascular occlusion on muscular function in humans. Journal of Applied Physiology, 88(6), pp.2097-2106.

Takarada, Y., 2003. Evaluation of muscle damage after a rugby match with special reference to tackle plays. British Journal of Sports Medicine, 37(5), pp.416-9.

Takarada, Y., Nakamura, Y., et al., 2000. Rapid increase in plasma growth hormone after lowintensity resistance exercise with vascular occlusion. Journal of Applied Physiology, 88(1), pp.61-5.

Takarada, Y., Sato, Y. \& Ishii, N., 2002. Effects of resistance exercise combined with vascular occlusion on muscle function in athletes. European Journal of Applied Physiology, 86(4), pp.308-314.

Tanaka, D. et al., 2016. Ischemic Preconditioning Enhances Muscle Endurance during Sustained Isometric Exercise. International Journal of Sports Medicine, pp.614-618.

Tanaka, H. et al., 1986. Persistent effects of a marathon run on the pituitary-testicular axis. Journal of Endocrinological Investigation, 9(2), pp.97-101.

Tanimoto, M., Madarame, H. \& Ishii, N., 2005. Muscle oxygenation and plasma growth hormone concentration during and after resistance exercise: Comparison between "KAATSU" and other types of regimen. International Journal of KAATSU Training Research, 1(2), pp.5156.

Tapuria, N. et al., 2008. Remote Ischemic Preconditioning: A Novel Protective Method From Ischemia Reperfusion Injury-A Review. Journal of Surgical Research, 150(2), pp.304-330.

Taylor, J.L. et al., 2000. Supraspinal fatigue during intermittent maximal voluntary contractions of the human elbow flexors. Journal of Applied Physiology, 89(1), pp.305-313.

Taylor, T. et al., 2014. The impact of neuromuscular electrical stimulation on recovery after intensive, muscle damaging, maximal speed training in professional team sports players. Journal of Science and Medicine in Sport, 18(3), pp.328-332.

Teixeira, E.L. et al., 2017. Blood flow restriction increases metabolic stress but decreases muscle activation during high-load resistance exercise. Muscle and Nerve, pp. 107-111.

Teo, W., McGuigan, M.R. \& Newton, M.J., 2011. The effects of circadian rhythmicity of salivary cortisol and testosterone on maximal isometric force, maximal dynamic force, and power output. Journal of Strength and Conditioning Research, 25(6), pp.1538-1545.

Teo, W., Newton, M.J. \& McGuigan, M.R., 2011. Circadian rhythms in exercise performance: Implications for hormonal and muscular adaptation. Journal of Sports Science and Medicine, 10(4), pp.600-606.

Thijssen, D.H.J. et al., 2016. Repeated ischaemic preconditioning: A novel therapeutic intervention and potential underlying mechanisms. Experimental Physiology, 101(6), pp.677-692.

Thomas, G.D. \& Victor, R.G., 1998. Nitric oxide mediates contraction-induced attenuation of 
sympathetic vasoconstriction in rat skeletal muscle. Journal of Physiology, 506(3), pp.817826.

Thorpe, R. \& Sunderland, C., 2012. Muscle Damage, Endocrine, and Immune Marker Response to a Soccer Match. Journal of Strength and Conditioning Research, 26(10), pp.2783-2790.

Tidball, J.G., 2005. Inflammatory processes in muscle injury and repair. American Journal of Physiology. Regulatory, Integrative and Comparative Physiology, 288(2), pp.R345-53.

Tocco, F. et al., 2015. Muscle Ischemic Preconditioning does not Improve Performance during Self-Paced Exercise. International Journal of Sports Medicine, 36(1), pp.9-15.

Trimble, M.H. \& Harp, S.S., 1998. Postexercise potentiation of the H-reflex in humans. Medicine \& Science in Sports \& Exercise, 30(6), pp.933-941.

Tsigos, C. \& Chrousos, G.P., 2002. Hypothalamic-pituitary-adrenal axis, neuroendocrine factors and stress. In Journal of Psychosomatic Research. pp. 865-871.

Turnes, T. et al., 2014. A fast-start pacing strategy speeds pulmonary oxygen uptake kinetics and improves supramaximal running performance. PLOS ONE, 9(10).

Twist, C. et al., 2012. Neuromuscular, biochemical and perceptual post-match fatigue in professional rugby league forwards and backs. Journal of Sports Sciences, 30(4), pp.359367.

Vaile, J. et al., 2011. Effect of cold water immersion on repeated cycling performance and limb blood flow. British Journal of Sports Medicine, 45(10), pp.825-829.

Vandavasi, M. \& Sukumar, C.D., 2016. Effect of exercise on blood pressure in athletes and untrained individuals. Journal of Contemporary Medicine and Dentistry, 4(2), pp.37-40.

Veldhuis, J.D., Keenan, D.M. \& Pincus, S.M., 2008. Motivations and methods for analyzing pulsatile hormone secretion. Endocrine Reviews, 29(7), pp.823-864.

Verwaerde, P. et al., 2002. The accuracy of the i-STAT portable analyser for measuring blood gases and $\mathrm{pH}$ in whole-blood samples from dogs. Research in Veterinary Science, 73(1), pp. $71-75$.

Vibarel-Rebot, N. et al., 2015. Oral contraceptive use and saliva diurnal pattern of metabolic steroid hormones in young healthy women. Contraception, 91(3), pp.245-247.

Vingren, J.L. et al., 2009. Effect of resistance exercise on muscle steroid receptor protein content in strength-trained men and women. Steroids, 74(13-14), pp.1033-1039.

Vingren, J.L. et al., 2012. Testosterone Physiology in Resistance Exercise and Training. Sports Medicine, 40(12), pp.1037-1053.

Vining, R.F. et al., 1983. Salivary cortisol: a better measure of adrenal cortical function than serum cortisol. Annals of Clinical Biochemistry, 20 (Pt 6)(2), pp.329-35.

Viru, A. \& Viru, M., 2004. Cortisol - Essential adaptation hormone in exercise. International Journal of Sports Medicine, 25(6), pp.461-464.

Vittek, J., et al., 1985. Direct radioimmunoassay (RIA) of salivary testosterone, correlation with 
free and total serum testosterone. Life Sciences, 37(8), pp.711-716.

Wang, C. et al., 1981. Salivary testosterone in men: Further evidence of a direct correlation with free serum testosterone. Journal of Clinical Endocrinology and Metabolism, 53(5), pp.10211024.

Wang, W.Z. et al., 2004. Ischemic Preconditioning-Induced Microvascular Protection at a Distance. Journal of Reconstructive Microsurgery, 20(2), pp.175-181.

Wang, W.Z., Baynosa, R.C. \& Zamboni, W.A., 2011. Therapeutic interventions against reperfusion injury in skeletal muscle. Journal of Surgical Research, 171(1), pp.175-182.

Warren, G.L. et al., 1993. Excitation failure in eccentric contraction-induced injury of mouse soleus muscle. Journal of Physiology, 468, pp.487-499.

Warren, G.L. et al., 1995. Redistribution of cell membrane probes following contraction-induced injury of mouse soleus muscle. Cell \& Tissue Research, 282(2), pp.311-320.

Warren, G.L., Lowe, D.A. \& Armstrong, R.B., 1999. Measurement tools used in the study of eccentric contraction-induced injury. Sports Medicine, 27(1), pp.43-59.

Waterhouse, J. et al., 2005. The circadian rhythm of core temperature: origin and some implications for exercise performance. Chronobiology international, 22(2), pp.207-225.

Weiss, L.W., Cureton, K.J. \& Thompson, F.N., 1983. Comparison of serum testosterone and androstenedione responses to weight lifting in men and women. European Journal of Applied Physiology and Occupational Physiology, 50(3), pp.413-419.

Wells, G.D., Selvadurai, H. \& Tein, I., 2009. Bioenergetic provision of energy for muscular activity. Paediatric Respiratory Reviews, 10(3), pp.83-90.

Wernbom, M. et al., 2009. Acute effects of blood flow restriction on muscle activity and endurance during fatiguing dynamic knee extensions at low load. Journal of Strength and Conditioning Research, 23(8), pp.2389-2395.

Wernbom, M., Augustsson, J. \& Thomeé, R., 2006. Effects of vascular occlusion on muscular endurance in dynamic knee extension exercise at different submaximal loads. Journal of Strength and Conditioning Research, 20(2), pp.372-377.

Weselcouch, E.O. et al., 1993. ATP-sensitive potassium channels and skeletal muscle function in vitro. The Journal of Pharmacology and Experimental Therapeutics, 267(1), pp.410-6.

West, D.J. et al., 2013. Influence of post-warm-up recovery time on swim performance in international swimmers. Journal of Science and Medicine in Sport, 16(2), pp.172-176.

West, D.J. et al., 2014. Neuromuscular function, hormonal, and mood responses to a professional rugby union match. Journal of Strength and Conditioning Research, 28(1), pp.194-200.

West, D.J. et al., 2011. Relationships between force-time characteristics of the isometric midthigh pull and dynamic performance in professional rugby league players. Journal of Strength \& Conditioning Research, 2(12), pp.3070-3075.

Wiegratz, I. et al., 2003. Effect of four different oral contraceptives on various sex hormones and 
serum-binding globulins. Contraception, 67(1), pp.25-32.

Wilson, J. et al., 2013. Practical blood flow restriction training increases acute determinants of hypertrophy without increasing indices of muscle damage. Jouranl of Strength and Conditioning Research, 27(11), pp.3068-3075.

Winget, C.M., DeRoshia, C.W. \& Holley, D.C., 1985. Circadian rhythms and athletic performance. Medicine \& Science in Sports \& Exercise, 17(5), pp.498-516.

Winkle, Van, D.M. et al., 1991. Natural history of preconditioning, cardioprotection depends on duration of transient ischemia \& time to subsequent ischemia. Coronary artery disease, 2(5), pp.613-619.

Wirth, M.M. \& Schultheiss, O.C., 2007. Basal testosterone moderates responses to anger faces in humans. Physiology and Behavior, 90(2-3), pp.496-505.

Wood, P., 2009. Salivary steroid assays - research or routine? Annals of clinical biochemistry, 46(Pt 3), pp.183-196.

Yamanaka, T., Farley, R.S. \& Caputo, J.L., 2012. Occlusion Training Increases Muscular Strength in Division IA Football Players. Journal of Strength and Conditioning Research, 26(9), pp.2523-2529.

Yamashita, N. et al., 1998. A "second window of protection" occurs $24 \mathrm{~h}$ after ischemic preconditioning in the rat heart. Journal of Molecular and Cellular Cardiology, 30(6), pp.1181-9.

Yasuda, T. et al., 2014. Effects of low-intensity, elastic band resistance exercise combined with blood flow restriction on muscle activation. Scandinavian Journal of Medicine and Science in Sports, 24(1), pp.55-61.

Yasuda, T. et al., 2009. Muscle activation during low-intensity muscle contractions with restricted blood flow. Journal of Sports Sciences, 27(5), pp.479-489.

Yasuda, T. et al., 2008. Muscle activation during low-intensity muscle contractions with varying levels of external limb compression. Journal of Sports Science \& Medicine, 7(4), pp.467-74.

Yasuda, T. et al., 2011. Relationship between limb and trunk muscle hypertrophy following highintensity resistance training and blood flow-restricted low-intensity resistance training. Clinical Physiology and Functional Imaging, 31(5), pp.347-51.

Yasuda, T. et al., 2010. Venous blood gas and metabolite response to low-intensity muscle contractions with external limb compression. Metabolism: Clinical and Experimental, 59(10), pp.1510-9.

Yellon, D.M. \& Downey, J.M., 2003. Preconditioning the myocardium: from cellular physiology to clinical cardiology. Physiological Reviews, 83(4), pp.1113-51.

Young, E.A., Abelson, J. \& Lightman, S.L., 2004. Cortisol pulsatility and its role in stress regulation and health. Frontiers in Neuroendocrinology, 25(2), pp.69-76.

Younger, A.S.E., McEwen, J. A \& Inkpen, K., 2004. Wide Contoured Thigh Cuffs and Automated 
Limb Occlusion Measurement Allow Lower Tourniquet Pressures. Clinical Orthopaedics and Related Research, 428(428), pp.286-293.

Zelles, T. et al., 1995. Concise Review: Saliva and Growth Factors: The Fountain of Youth Resides in Us All. Journal of Dental Research, 74(12), pp.1826-1832.

Zhao, Z.Q. \& Vinten-Johansen, J., 2006. Postconditioning: Reduction of reperfusion-induced injury. Cardiovascular Research, 70(2), pp.200-211.

Zimmerman, Y. et al., 2014. The effect of combined oral contraception on testosterone levels in healthy women: A systematic review and meta-analysis. Human Reproduction Update, 20(1), pp.76-105. 


\section{Appendices}

Appendix A: Ethical Approval

The effect of morning exercise with and without occlusion on afternoon performance in elite athletes

The use of intermittent lower-limb occlusion to enhance recovery after repeated sprint running performance - PG/2014/33

The effect of early versus delayed IPC on swimming performance - REC 2016-093A 
Appendix B: Participant information sheet

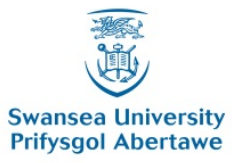

\author{
Applied Sports Technology Exercise and Medicine Research Centre (A-STEM) \\ Sport and Health Portfolio, College of Engineering
}

PARTICIPANT INFORMATION SHEET

(Version 1.1, Date: )

\title{
Project Title:
}

The effect of morning exercise with and without occlusion on afternoon performance in elite athletes

\section{Contact Details:}

Miss Natalie Williams;

\section{Invitation Paragraph}

We would like to invite you to volunteer for this study that aims to identify if the use of exercise and exercise with blood flow restriction will help with physiological preparation and readiness for competition performance. The study will be beneficial to you, aiming to improve performance as assessed through a countermovement jump and isometric mid-thigh pull that can be transferred into competition. We would like to thank you for taking the time to read this information sheet and very much hope you decide to take part.

\section{What is the purpose of the study?}

The purpose of the study is to identify if the use of morning exercise both with and without blood flow occlusion, can be used as a pre-competition strategy to benefit your afternoon performance.

\section{Why have I been chosen??}

You have been chosen to complete this study based upon the level and experience of competition performance, combined with your previous training history. You will have the right to withdraw from the study at any time, without having to provide reasoning.

\section{What will happen to me if I take part?}

You will be required to attend 4 sessions; the sessions will require you to perform 4 conditions. These conditions involve morning exercise at 3RM, morning exercise combined with blood flow occlusion and occlusion only without exercise. The first two conditions require you to complete maximal effort CMJ and isometric mid-thigh pull (IMTP), therefore participating in strenuous exercise. Several measures will be taken during the session including saliva samples, capillary blood samples, $\mathrm{O}_{2}$ saturation and blood pressure. The exercise and occlusion will be performed in the morning and you will then be requested to return $4-6 \mathrm{~h}$ post to complete the CMJ and IMTP. 


\section{What are the possible disadvantages of taking part?}

There are possible risks of the study. Firstly, blood samples and saliva will be collected. Procedures of how both will be collected will be explained to you prior to taking the blood and saliva and you will have the right to withdraw at any stage. The blood flow occlusion through use of blood pressure cuffs may cause discomfort, therefore you will be given the option not to participate if you are not comfortable and likewise have the right to withdraw if you experience any pain or discomfort such as light-headedness and nausea during the study. You will also be expected to participate in strenuous exercise that may cause some slight discomfort, but should not be any different from your normal training and competition.

\section{What are the possible benefits of taking part?}

As a result of participating, you will gain valuable information on potential pre-competition strategies that can be used prior to competing and may be beneficial to your performance.

\section{Will my taking part in the study be kept confidential?}

All data will be kept confidential and results will not be accessible to anyone else, only the research team will have access to the information. At the end of the study all data will be disposed of according to set guidelines.

\section{What if I have any questions?}

If you have any questions throughout the duration of this study, please do not hesitate to ask contact details for the researcher are provided at the start of this document. 
Applied Sports Technology Exercise and Medicine Research Centre (A-STEM)

Sport and Health Portfolio, College of Engineering

\section{PARTICIPANT INFORMATION SHEET}

(Version 1.1: Date:)

\section{Project Title:}

The effect of early versus delayed IPC on swimming performance

\section{Contact Details:}

Miss Natalie Williams;

\section{Invitation Paragraph}

We would like to invite you to volunteer for this study that aims to measure response to a pre-competition strategy, aiming to improve swimming performance. As a swimmer, you will have the opportunity to try a pre-competition strategy called ischemic preconditioning, testing it at different time points before completing a 100 or $200 \mathrm{~m}$ stroke specific time trial (TT). We would like to thank you for taking the time to read this information sheet and very much hope you decide to take part.

\section{What is the purpose of the study?}

The purpose of the study is to monitor your response to a pre-competition strategy called ischemic preconditioning (IPC) and see how it affects your performance when it is used at different time points before a swimming time trial performance.

\section{Why have I been chosen?}

You have been chosen to complete this study based upon the level and experience of competition performance, combined with your previous training history. You will have the right to withdraw from the study at any time, without having to provide reasoning. All information collected will be anonymous to practitioners and coaches and therefore your decision to participate or withdraw will not affect future squad/competition selection or access to sport science and coaching support.

\section{What will happen to me if I take part?}

Ischemic preconditioning involves the use of inflatable cuffs being wrapped around your thighs and being inflated and deflated. Specifically, for this monitoring you will be required to complete 3 sessions, these will involve 2 trials when the cuffs are applied and inflated $2 \mathrm{~h}$ before a swimming time trial, the other session will involve having cuffs applied and inflated $24 \mathrm{~h}$ before a swimming time trial. 
Once the cuffs are wrapped around your thighs, you will be asked to lie down and the cuffs will be inflated for $5 \mathrm{~min}$ and deflated for $5 \mathrm{~min}$, repeated a total of 4 times (total time lying down will be 40 minutes).

You will then be asked to rest according to the condition being complete ( $2 \mathrm{~h}$ or $24 \mathrm{~h}$ ); 1 $\mathrm{h}$ before swimming time trial, you will be asked to complete a swimming warm up, followed by 20 min rest. A maximal effort swimming time trial (100 or $200 \mathrm{~m}$ stroke specific) will immediately follow and will be complete individually. Your time trial will be videoed to allow for your stroke rate, stroke length, split times, time and distance underwater off the start and turns and total time to be recorded.

No exercise is to be complete $24 \mathrm{~h}$ prior to testing and rest between the completion of cuff inflations and swimming time trial.

Measures that you will be asked to complete, please note you can choose to opt out of any of these if you are not comfortable, however please be aware opting out of blood pressure measurement will prevent participation.

Blood Pressure at rest - you will be asked to rest for $10 \mathrm{~min}$. Following this the procedure will be explained to you, including making you aware that there may be a tight feeling and very minor discomfort. You will be asked to take a seat with the arm at heart level. You will be asked to have your feet on the floor, uncrossed and your back will be supported in the chair. Once in this position, the cuff size will be checked and wrapped around your upper arm. A blood pressure monitor will be used to measure your blood pressure.

Capillary blood sample - Prior to any sample being taken you will be asked to be seated, the procedure will be explained to you and confirm you are happy to proceed. The area of the finger will be cleaned with an alcohol wipe before a capillary blood sample is taken from your finger, a single use lancet will be used to puncture the tip of the finger in which a blood sample will be collected from.

Heart Rate (HR) - this will be recorded using a polar heart rate monitor. You will be asked to rest for $10 \mathrm{~min}$ on arrival to record baseline $\mathrm{HR}$ and will be monitored and data collected throughout the whole session.

You will also be asked your rating of perceived exertion on completion of the swimming time trial.

\section{What are the possible disadvantages of taking part?}

There are some possible disadvantages of taking part. Firstly, capillary blood (from your finger) will be taken. Procedures of how blood will be collected will be explained to you prior to taking any samples and you will have the right to withdraw at any time. You may experience some discomfort from the cuffs such as pain, pins and needles and light headiness. This will be explained to you before cuffs are wrapped around your legs inflated and you will be asked to let the researcher know immediately if you feel any symptoms when cuffs are inflated, researchers will continue to ask you throughout the protocol as well. If any symptoms are experienced the cuffs will be deflated and removed 
immediately. You will also be expected to participate in strenuous exercise, completing 3 swimming time trials in total.

\section{What are the possible benefits of taking part?}

Because of participating, you will gain valuable information on a pre-competition strategy that may potentially improve performance.

\section{Will my taking part in the study be kept confidential?}

All data will be kept confidential and results will not be accessible to anyone else. You and your coach (Mark Rose or Mark Skimming) will receive group average results. At the end of the study all data will be disposed of after 5 years.

\section{What if I have any questions?}

If you have any questions throughout the duration of this study, please do not hesitate to ask - contact details for the researcher are provided at the start of this document. 


\section{Applied Sports Technology Exercise and Medicine Research Centre (A-STEM)}

Sport and Health Portfolio, College of Engineering

\section{PARTICIPANT INFORMATION SHEET}

(Version 1.1, Date:)

\section{Project Title:}

The use of intermittent lower-limb occlusion to enhance recovery after repeated sprint running performance

\section{Contact Details:}

Miss Natalie Williams;

\section{Invitation Paragraph}

We would like to invite you to volunteer for this study that aims to identify if the use of blood flow restriction, also termed occlusion, assists with recovery after completing repeated sprints. The study will be beneficial to you, aiming to improve your recovery as assessed through a countermovement jump and specific blood markers that indicate recovery following strenuous exercise. We would like to thank you for taking the time to read this information sheet and very much hope you decide to take part.

\section{What is the purpose of the study?}

The purpose of the study is to identify if the use of blood flow occlusion can increase your recovery, to be used after training and/or competition to prepare for the next bout of exercise.

\section{Why have I been chosen??}

You have been chosen to complete this study based upon the level and experience of competition performance, combined with your previous training history. You will have the right to withdraw from the study at any time, without having to provide reasoning. All information collected will be anonymous to Sport Wales practitioners and coaches and therefore your decision to participate or withdraw will not affect future squad/competition selection or access to sport science support.

\section{What will happen to me if I take part?}

You will be required to attend 2 sessions; the sessions will require you to complete 2 conditions. Both conditions will involve providing baseline measures including saliva and capillary blood samples and completing maximal effort countermovement jump. A repeated sprint protocol $(15 \times 30 \mathrm{~m})$ will then be complete prior to you being asked to lie down for $12 \mathrm{~min}$ and have blood pressure cuffs applied to the highest point of your thigh. This will be inflated to $15 \mathrm{mmHg}$ or an individualized cuff pressure alternating between each leg for $3 \mathrm{~min}$; the cycle will be repeated twice for a total of $12 \mathrm{~min}$ (total $6 \mathrm{~min}$ for each leg). The cuffs will contain a pneumatic bag along its inner surface that is connected to a pressure gauge that will be manually inflated. Following this baseline measures will be repeated again 2, 24 and $48 \mathrm{~h}$ post 
cessation of exercise. Below provides you with information on the procedures involved in the measures being collected.

Blood Pressure - this will be complete in a private room, you will be asked to rest for $10 \mathrm{~min}$. Following this the procedure will be explained to you, including making you aware that there may be a tight feeling and very minor discomfort. You will be asked to take a seat with the arm at heart level, the same arm will be used for measurement across both testing days, a pillow will be available to reduce any discomfort or change the height of the arm to ensure it is at the level of the heart. You will be asked to have your feet on the floor, uncrossed and your back will be supported in the chair. Once this position has been established, the cuff size will be checked and applied to your upper arm. An automated blood pressure monitor will be used to determine your blood pressure: 2 readings will be taken. Blood pressure measurements will also be taken after exercise and after occlusion.

Capillary blood sample - Prior to any sample being taken you will be asked to be seated, the procedure will be explained to you and confirm you are happy to proceed. The area of the finger will be cleaned with an alcohol wipe before a capillary blood sample is taken from your finger, a single use lancet will be used to puncture the tip of the finger in which a blood sample will be collected from.

Saliva sample - you will be asked to provide a $2 \mathrm{ml}$ sample of saliva into a sterile container before beginning, immediately after repeated sprints and also 2, 24 and $48 \mathrm{~h}$ post, these are the same time points as the blood samples. This method is not invasive but the procedure will be explained to you and confirmation you are happy prior to providing a saliva sample.

In addition to above measurements, you will be asked to complete a countermovement jump which will involve standing on a force platform, hands on your hips, before dipping to a selfselected depth and then exploding upwards in an attempt to achieve maximum height. You will be required to complete 3 maximum jumps with 1.5 min rest between efforts. You will also be asked to complete an Isometric Mid-thigh pull (IMTP) test, you will be asked to assume a body position similar to that of athletes completing the second pull of a power clean with a flat trunk position and shoulders in line with the bar. This position will allow you to maintain a knee angle of approximately $120-130^{\circ}$. The bar height can be fixed at various heights above the force platform, to accommodate different heights, once the bar height is established, you will be instructed to pull as hard and as fast as possible on the bar for a period of approximately 5 seconds.

Participants will be asked to refrain from exercise and caffeine the morning prior to testing. Food consumption will also be controlled with no food or fluid consumption $2 \mathrm{~h}$ prior to saliva samples.

\section{What are the possible disadvantages of taking part?}

There are possible risks of the study. Firstly, blood samples and saliva will be collected. Procedures of how both will be collected will be explained to you prior to taking the blood and saliva and you will have the right to withdraw at any stage. The blood flow occlusion through use of blood pressure cuffs may cause discomfort, therefore you will be given the option not to participate if you are not comfortable and likewise have the right to withdraw if you experience any pain or discomfort such as light-headedness and nausea during the study. You will also be expected to participate in strenuous exercise that may cause some slight discomfort, but should 
not be any different from your normal training and competition. All results will be anonymous to Sport Wales and coaches and therefore if you decide not to participate or withdraw it will not influence further access to sport science support or selection for future squads/competitions.

\section{What are the possible benefits of taking part?}

As a result of participating, you will gain valuable information on potential recovery strategies that can be after training or competing.

\section{Will my taking part in the study be kept confidential?}

All data will be kept confidential and results will not be accessible to anyone else, only the research team will have access to the information. At the end of the study all data will be disposed of according to set guidelines.

\section{What if I have any questions?}

If you have any questions throughout the duration of this study, please do not hesitate to ask contact details for the researcher are provided at the start of this document. 
Appendix C: Consent form and health screening questionnaire

\section{PARTICIPANT CONSENT FORM \\ (Version 1.1, Date:)}

\section{Project Title:}

\section{Contact Details:}

Please initial box

1. I confirm that I have read and understood the information sheet dated ................... (version number .) for the above study and have had the opportunity to ask questions.

2. I understand that my participation is voluntary and that I am free to withdraw at any time, without giving any reason, without my medical care or legal rights being affected.

3. I understand that sections of any of data obtained may be looked at by responsible individuals from the Swansea University or from regulatory authorities where it is relevant to my taking part in research. I give permission for these individuals to have access to these records.

4. I agree to take part in the above study.

Name of Participant

Name of Person taking consent
Date

Date

Date
Signature

Signature

Researcher

Signature 


\section{AHA/ACSM Health/Fitness Facility Preparticipation Screening Questionnaire}

Assess your health status by marking all true statements

History

You have had:

\author{
a heart attack \\ heart surgery \\ cardiac catheterization \\ _ coronary angioplasty (PTCA) \\ _ pacemaker/implantable cardiac \\ __ defibrillator/rhythm disturbance \\ heart valve disease \\ __ heart failure \\ heart transplantation \\ __ congenital heart disease
}

\section{Symptoms}

You experience chest discomfort with exertion.

You experience unreasonable breathlessness.

You experience dizziness, fainting, or blackouts.

You take heart medications.

\section{Other health issues}

You have diabetes.

You have asthma or other lung disease.

- You have burning or cramping sensation in your lower

legs when walking short distances.

You have musculoskeletal problems that limit your

physical activity.

You have concerns about the safety of exercise.

You take prescription medication(s).

You are pregnant.
If you marked any of these statements in this section, consult your physician or other appropriate health care provider before engaging in exercise. You may need to use a facility with a medically qualified staff.

\section{Cardiovascular risk factors}

You are a man older than 45 years.

You are a woman older than 55 years,

have had a hysterectomy, or are postmenopausal.

You smoke, or quit smoking within the previous 6 months.

Your blood pressure is $>140 / 90 \mathrm{~mm} \mathrm{Hg}$.

You do not know your blood pressure.

You take blood pressure medication.

Your blood cholesterol level is $>200 \mathrm{mg} / \mathrm{dL}$.

You do not know your cholesterol level.

You have a close blood relative who had a heart attack

or heart surgery before age 55 (father or brother) or age 65 (mother or sister).

You are physically inactive (i.e., you get $<30$ minutes of

physical activity on at least 3 days per week).

You are $>20$ pounds overweight.

If you marked two or more of the statements in this section you should consult your physician or other appropriate health care provider before engaging in exercise. You might benefit from using a facility with a professionally qualified exercise staff to guide your exercise program.
None of the above
You should be able to exercise safely without consulting your physician or other appropriate health care provider in a selfguided program or almost any facility that meets your exercise program needs. 
Appendix D: Warm up for countermovement jump

Exercise

Light jog

Skip

Lunge and twist (jog back)

Squat and turn (jog back)

Side to side (1 forwards, 1 backwards)

Double squat (jog back)

Dynamic Stretches

Spiderman

Kneeling Rec Fem stretch

Scorpions

Side lunge

Body weight squats (hands on hips)

Body weight jump squats (hands on hips)
Reps/Time

4

2

1

1

2

2

10

10

10

10

5

5 
Appendix E: Warm up prior to swimming time trial performance

\begin{tabular}{ll}
\hline Distance $(\mathrm{m})$ & Prescription \\
\hline $1 \times 600$ & Mixed stroke \\
$4 \times 150$ & 50 kick, 50 drill, 50 swim \\
$3 \times 100$ & Descend $1-3$ to $20 / 30 \mathrm{bbm}$ on 1.40 \\
$12 \times 50$ & Perfect stroke on 60 \\
& 15m fast on 60 \\
& Descend $1-3200$ pace $+2,1,0$ on 60 \\
& Steady on 60 \\
\hline
\end{tabular}

bbm - beats below max 
Appendix F: Borg Scale

\begin{tabular}{cc}
\hline Rating & Perceived Exertion \\
\hline 6 & No exertion \\
\hline 7 & Extremely light \\
\hline 8 & \\
\hline 9 & Very light \\
\hline 10 & \\
\hline 11 & Light \\
\hline 12 & \\
\hline 13 & Somewhat hard \\
\hline 14 & \\
\hline 15 & Hard \\
\hline 16 & \\
\hline 17 & Very hard \\
\hline 18 & \\
\hline 19 & Extremely hard \\
\hline 20 & Maximal exertion \\
\hline \hline
\end{tabular}


Appendix G: Perception of muscle soreness likert scale

Please tick the sentence below that best describes your current muscle soreness during normal movement:

[ ]0 A complete absence of soreness

[] 1 A light pain felt only when touched / a vague ache

[ $]_{2}$ A moderate pain felt only when touched/a slight persistent pain

[ ] 3 A light pain when walking up or down stairs

[1/4 A light pain when walking on a flat surface / painful

[15 A moderale pain, stiffness or weakness when walking / very painful

[16 A severe pain that limits my ability to move 
Appendix H: Perception recovery likert scale

Please circle the sentence below that best describes your recovery status:

10 Very well recovered/ Highly energetic

9

8 Well recovered / Somewhat energetic

7

6 Moderately recovered

5 Adequately recovered

4 Somewhat recovered

3

2 Not well recovered / Somewhat tired

1

0 Very poorly recovered / Extremely tired
Expect Improved Performance

Expect Similar Performance

Expect Declined Performance 